\title{
VIBRO-ACOUSTICAL INSTABILITIES INDUCED BY COMBUSTION DYNAMICS IN GAS TURBINE COMBUSTORS
}

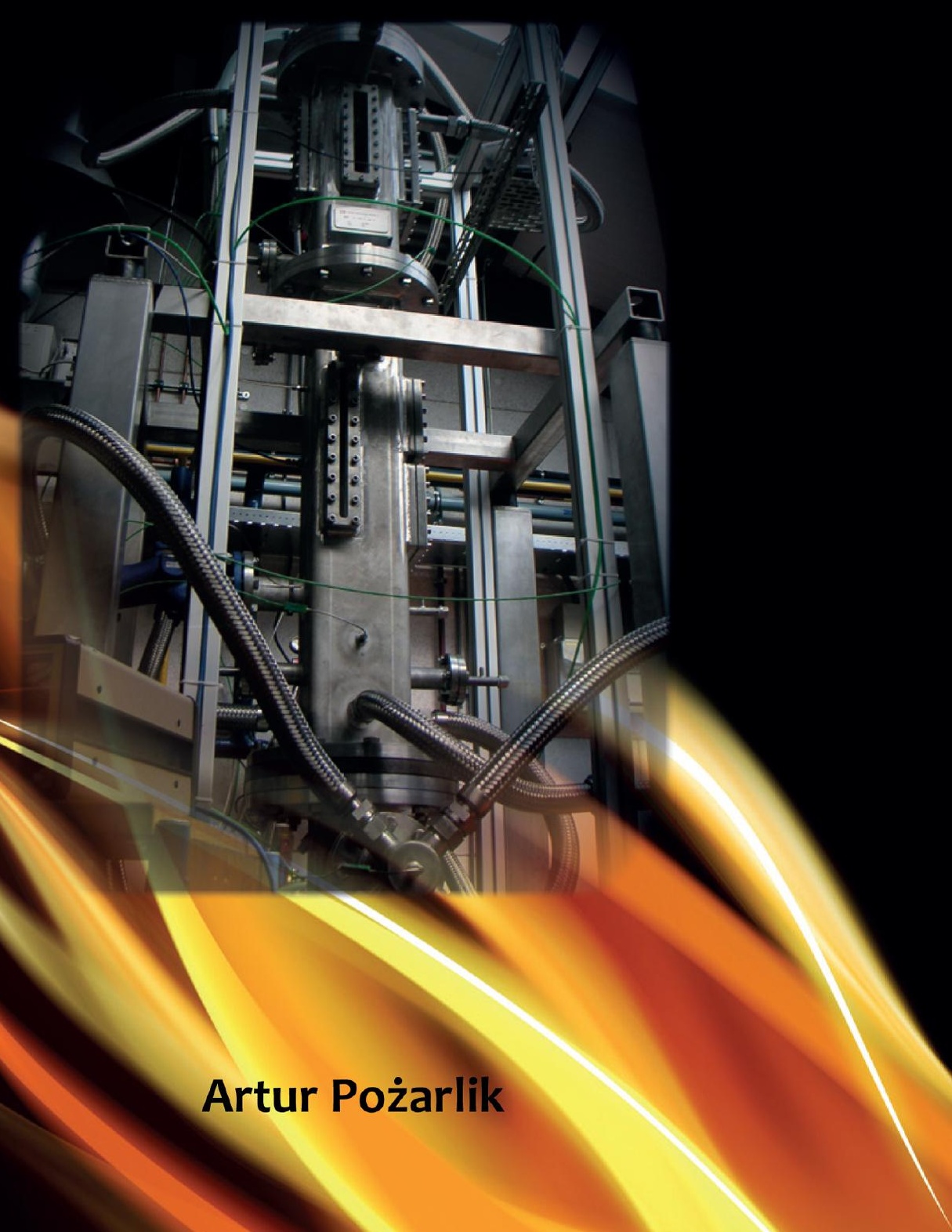




\section{VIBRO-ACOUSTICAL INSTABILITIES INDUCED BY COMBUSTION DYNAMICS IN GAS TURBINE COMBUSTORS}

Artur Pożarlik 


\section{Composition of the graduation committee}

Chairman and secretary

Prof.dr. F. Eising

University of Twente

Promotor

Prof.dr.ir. T.H. van der Meer

University of Twente

Assistant promotor

Dr.ir. J.B.W. Kok

University of Twente

Members

Prof.dr.ir. A. de Boer

University of Twente

Prof.dr.ir. A. Hirschberg

University of Twente

Prof.dr. L.P.H. de Goey

Eindhoven University of Technology

Prof.dr.ir. N.B. Roozen

Eindhoven University of Technology

Dr. A. Morgans

Imperial College London

The research was performed in the framework of the EU Marie Curie TN project FLUISTCOM, contract number: MRTN-CT-2003-504183.

Vibro-acoustical instabilities induced by combustion dynamics in gas turbine combustors

Pożarlik, Artur Krzysztof

$\mathrm{PhD}$ thesis, University of Twente, Enschede, The Netherlands

December 2010

ISBN: 978-90-365-3126-9

Keywords: fluid-structure interaction, thermo-acoustic instabilities, combustion, vibration

Copyright $(2010$ by A.K. Pożarlik, Enschede, The Netherlands

Cover: Photograph of DESIRE setup, taken by J.F. van Kampen/N. Dijkstra 


\title{
VIBRO-ACOUSTICAL INSTABILITIES INDUCED BY COMBUSTION DYNAMICS IN GAS TURBINE COMBUSTORS
}

\author{
DISSERTATION
}

to obtain

the degree of doctor at the University of Twente,

on the authority of the rector magnificus,

prof.dr. H. Brinksma,

on account of the decision of the graduation committee,

to be publicly defended

on Friday $3^{\text {rd }}$ of December 2010 at 15.00

by

Artur Pożarlik

born on January $3^{\text {rd }}, 1977$

in Radomsko, Poland 
The dissertation is approved by:

Prof.dr.ir. T.H. van der Meer

Dr.ir. J.B.W. Kok

\section{Promotor}

Assistant promotor 
To my parents and my wife Magdalena 



\section{SUMMARY}

Introduction of lean premixed combustion for power and heat generation reduced significantly emission of $\mathrm{NO}_{\mathrm{x}}$ pollutants to atmosphere. However, this type of combustion suffers from a high sensitivity to thermo-acoustic instabilities which may occur in a combustion chamber of a gas turbine. Pressure fluctuations induced by instabilities may exceed the level of $140 \mathrm{~dB}$. This level of acoustic excitation is hazardous to the combustion chamber walls called liner. Due to the high amplitude of pressure oscillations fatigue damage may occur. The situation is even worse when mutual interaction between thermoacoustic instabilities and liner vibration is present. Both processes enhance each other up to the moment when the pressure saturation limit is obtained. This behaviour reduces the life time of the gas turbine significantly.

Crucial for the combustion system is to operate at stable combustion regime. The possibilities of thermo-acoustic interaction to appear must be predicted in advance. It has to be known whether at given operating conditions instabilities may occur, what will be their frequency and amplitude, whether they are coupled with combustion modes and what is their effect on the liner vibrations. A feedback loop between thermo-acoustic instabilities and liner vibrations is also an important factor for prediction.

This multi-phenomena interaction is presented and studied in this thesis. The experimental and numerical techniques are employed to investigate the interaction between coupled fields. The experimental part of the study is done on the laboratory scale combustion test rig, which mimics the combustion conditions as encountered in the full scale gas turbine. Non-intrusive techniques for flame behaviour, pressure fluctuations and liner vibration measurement are used. Experiments are performed at operating conditions which differ with respect to power and absolute pressure, using two different liner configurations. The obtained results are used for validation of numerical models.

Two main streams of numerical computations are used. In the first one, the thermo-acoustic instabilities are linked to walls vibration using partitioning approach. Here two numerical solvers using Finite Volume Method (Ansys-CFX) and Finite Element Method (AnsysMultiphysics) are employed to calculate phenomena occurring in the fluid and structural domain, respectively. These solvers exchange information about mechanical loads and structural displacement every time step through interface connection created between them. Both one-way and two-way data transfer is studied. To reduce the computational effort, 
only a weak, serial coupling between interacting fields is done. This analysis is referred further in the thesis as a fluid-structure interaction (FSI).

In the acousto-elastic analysis a hybrid approach is used. Here, first the CFD flow is calculated in the Ansys-CFX code and latter a pressure data from the near-flame region is transferred to FEM code as input conditions. This solution allows to solve the acoustic waves inside the combustion chamber more precise than the FSI model, but in costs of only one-way interaction between acoustic wave and flame. Within the acousto-elastic analysis also modal analysis of acoustic, structural and coupled modes is performed.

The results of all numerical models have shown a good agreement with experimental data. Both, fluid-structure interaction model and acousto-elastic model predicted correctly the frequency of thermo-acoustic instabilities as well as main vibration frequency of the liner. The amplitude of the numerical signal was under-predicted with respect to data recorded during experiment due to short total calculation time and numerical dissipation and dispersion of acoustic wave inside the CFD code. The observed feedback from the vibrating walls to acoustic field inside the combustion chamber was minor. Furthermore, modal analysis of coupled modes pointed out correctly the frequencies at which instabilities may occur. However, in this case further FSI or AE analysis must be done to confirm whether at given operating conditions instabilities are present.

In the last part of the thesis a backward facing step model is presented with pulsating flow conditions and vibrating bottom wall. These investigations give insigne into the effect of pulsating conditions on the heat transfer through the walls. The results have shown that in the current setup configuration, the frequency of thermo-acoustic instabilities and wall vibrations have only minor effect on the mean Stanton number. 


\section{CONTENTS}

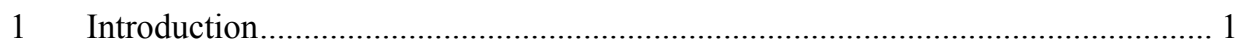

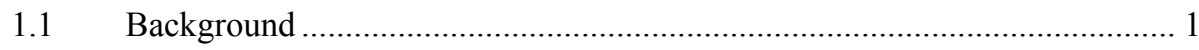

1.2 Lean Premixed Combustion ....................................................................... 2

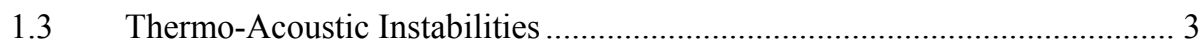

1.4 Instabilities In The Combustion System................................................... 5

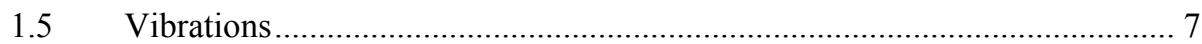

1.6 Research Objective...................................................................... 8

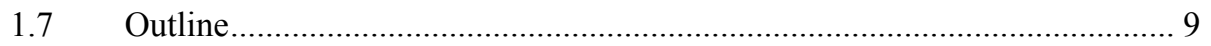

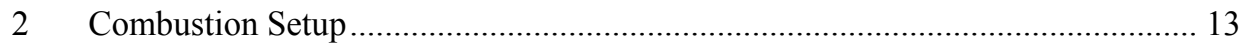

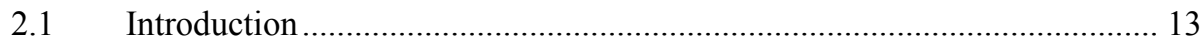

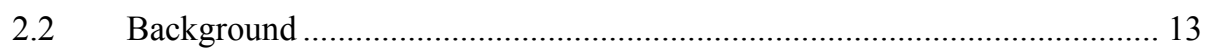

$2.3 \quad$ Structural Section ........................................................................... 17

$2.4 \quad$ Laser Doppler Vibrometer .................................................................. 19

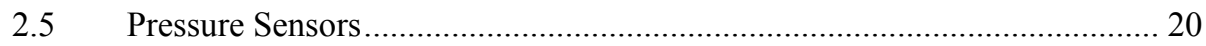

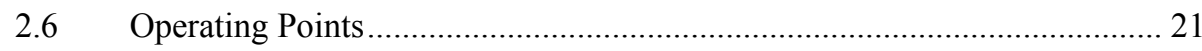

$2.7 \quad$ Steady and Pulsating Measurements ....................................................... 22

2.7.1 Steady Flow Results.............................................................................. 23

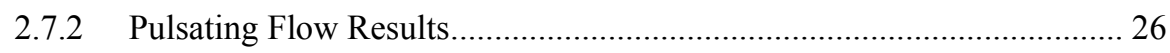

$2.8 \quad$ Flame Transfer Function ........................................................................ 28

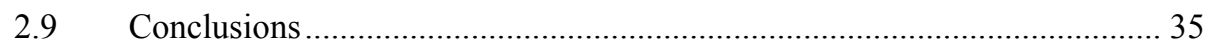




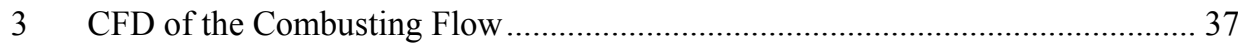

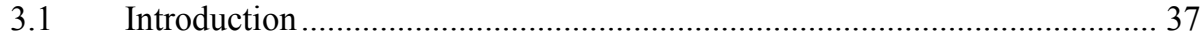

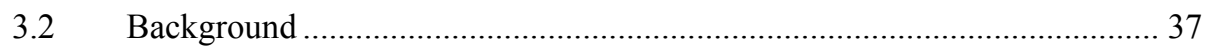

3.3 Turbulent Combustion........................................................................... 39

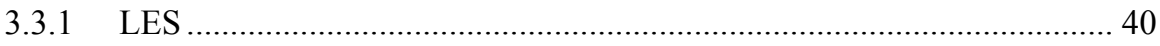

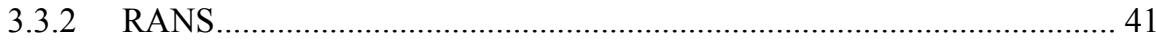

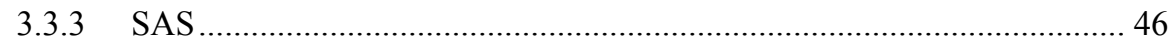

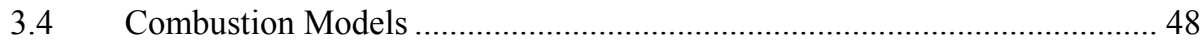

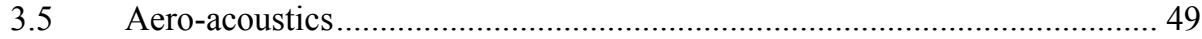

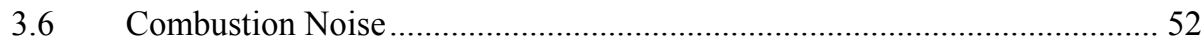

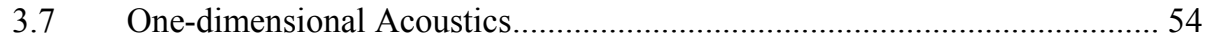

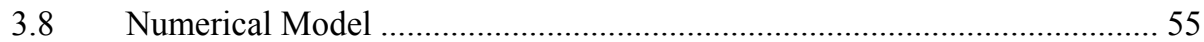

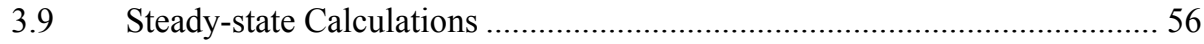

3.9.1 Adiabatic vs Non-adiabatic Wall .......................................................... 57

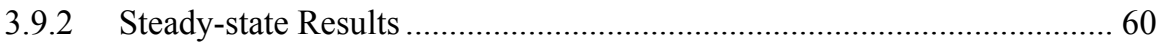

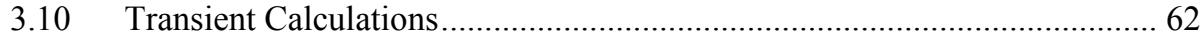

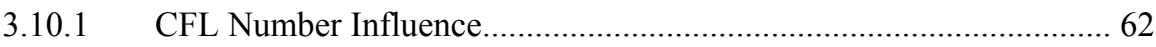

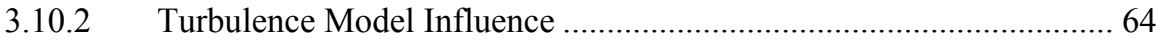

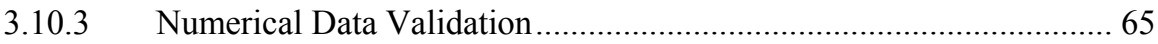

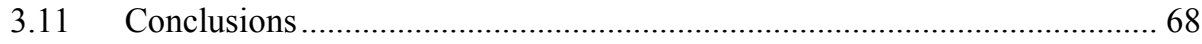

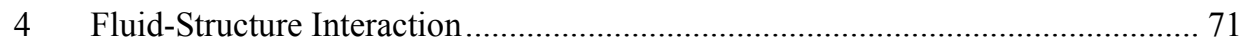

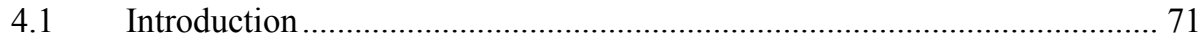

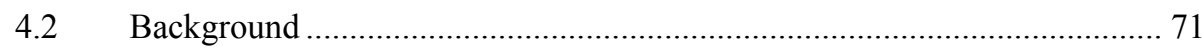

4.2.1 Monolithical vs Partitioned Approach ................................................... 72

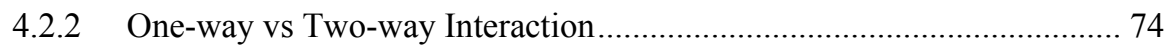

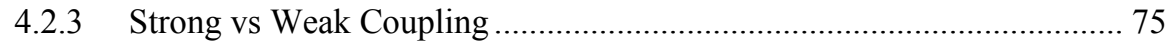

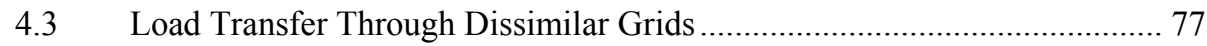

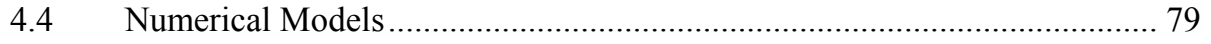




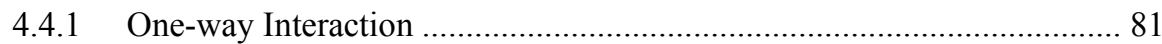

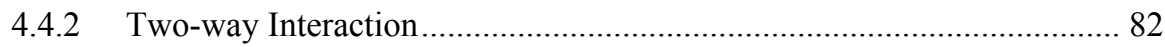

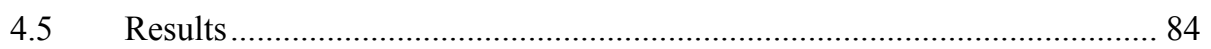

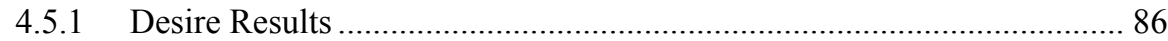

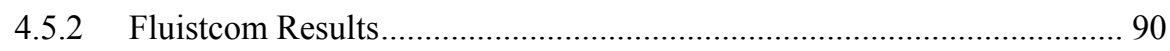

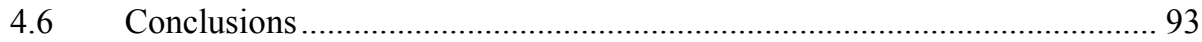

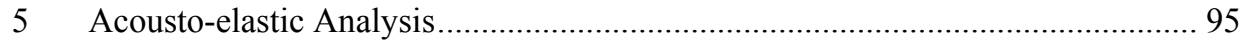

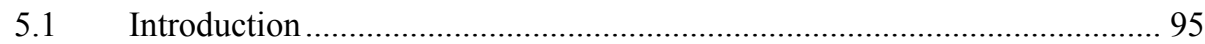

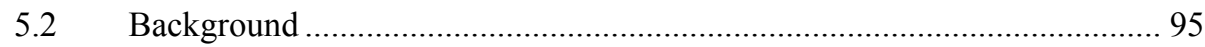

5.2.1 Air Influence On System Behaviour...………………………………... 96

5.2.2 Near and Far Field Acoustics.................................................................. 98

5.2.3 Propagating and Evanescent waves ..................................................... 99

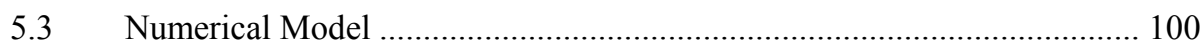

5.3.1 Geometry and Boundary Conditions...................................................... 103

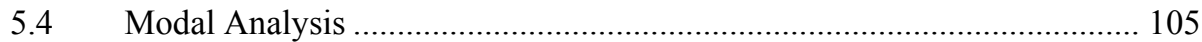

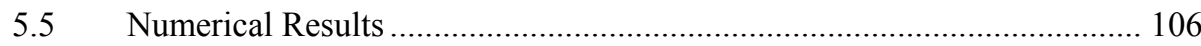

5.5.1 Desire - Acousto-elastic Analysis ....................................................... 106

5.5.2 Fluistcom - Acousto-elastic Analysis................................................... 110

5.5.3 Modal Analysis of Desire …………………………………………........ 113

5.5.4 Modal Analysis of Fluistcom.................................................................. 117

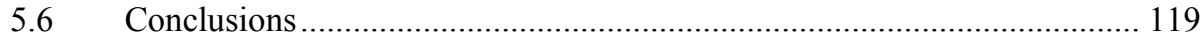

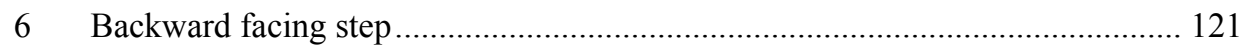

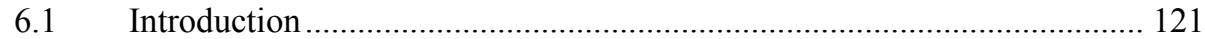

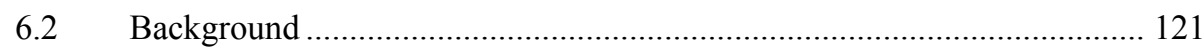

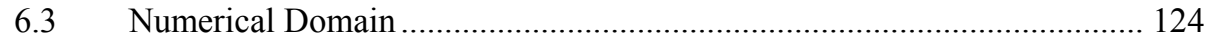

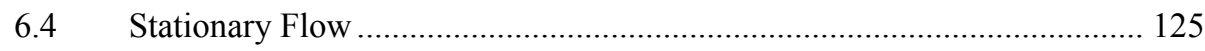

6.5 Transient Flow - Inlet Velocity Pulsation.................................................... 128 
6.6 Transient Flow - Oscillating Wall ........................................................... 132

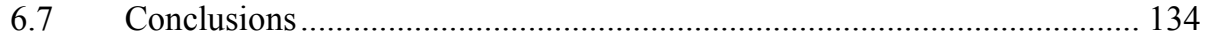

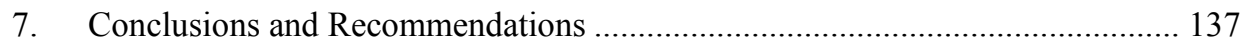

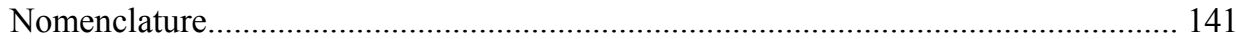

Appendix A - Numerical and Analytical Model of a Plate........................................... 147

Appendix B - Natural Frequencies of the Liner........................................................... 149

Appendix C - Stress and Displacement Analysis....................................................... 155

Appendix D - Experiment with Instabilities at First Acoustic Mode............................. 157

Appendix E - CFI Model ........................................................................................ 159

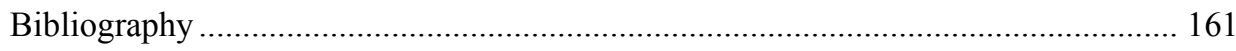

Acknowledgments ................................................................................................ 171 


\section{INTRODUCTION}

\subsection{BACKGROUND}

Nowadays, production of heat and power to fulfil human requirements is one of the main tasks for the industry. About $90 \%$ of the total produced power, comes from combustion of fossil fuels, see (Laherrere, 2006). During a burning process of hydrocarbon fuels not only power and heat are produced but also harmful products of chemical reactions taking place during the combustion are released. These pollutants are emitted directly to the atmosphere. Since during the developing process people hardly thought about their planet, the Earth now is significantly contaminated by pollution coming from the human activities. To prevent further degradation of our natural environment, on $11^{\text {th }}$ of December 1997 in Kyoto, the leaders of many countries signed the Kyoto protocol. This is an agreement to reduce significantly the emission of harmful species to the environment. The full text of this document is available on (UNFCCC, 1997). One of the most important components of the pollutants, are oxides of nitrogen, also known as NOx. Since NOx are formed mainly during high temperature processes, clean combustion technologies had to be developed for energy generation. Lean premixed combustion of natural gas is one of them, see (Turns, 1996), (Warnatz, Maas, \& Dibble, 2001).

Within the scope of this thesis, the lean premixed combustion process of natural gas inside a combustion chamber of a gas turbine engine is investigated. This research is a continuation of research done by (Huls, 2006) and (Van Kampen, 2006). In the present work the main focus is placed on the mutual interaction between the combustion process, acoustics and vibrations of the combustion chamber walls, called liner; which extends and goes beyond the investigations done by aforementioned researchers. Coupling of all the interacting processes and prediction of hazardous frequencies which may lead to the system destruction are of primary interest. For this purpose new models describing the complexity of the mutual dependence between interacting fields are designed. 
The work presented here is done within the Marie Curie TN project FLUISTCOM (project number: MRTN-CT-2003-504183). The collaborative research project FLUISTCOM is one of the first Marie Curie Research and Training Networks funded by the European commission within the 6th Framework Programme. Of relative small size (6 partners: CERFACS, CIMNE, DLR, Queen's University of Belfast, SIEMENS and University of Twente, from 5 countries), FLUISTCOM belongs to the engineering panel and represents an initiative to strengthen the fundamental scientific work in the multidisciplinary engineering field of fluid-structure interaction for turbulent combustion systems.

\subsection{Lean Premixed Combustion}

The idea behind the lean premixed combustion process is to reduce the combustion temperature significantly by using much more air than is necessary to oxidize fuel completely. This way the same amount of heat is released as under stoichiometric conditions to heat up more air, resulting in lower combustion temperature. A decrease in the combustion temperature does not have an influence on the engine efficiency since the combustion gases produced have to be cooled down to temperatures much lower than the stoichiometric flame temperature, in order to reach a temperature that the turbine blades can survive without deformation or melting. Furthermore, fuel and air should be perfectly premixed before they enter the burner mouth. This prevents occurrence of hot spots, after ignition, where the ratio of fuel to air is higher than desirable. An example of a typical engine used with the lean combustion is presented in Figure 1-1. 


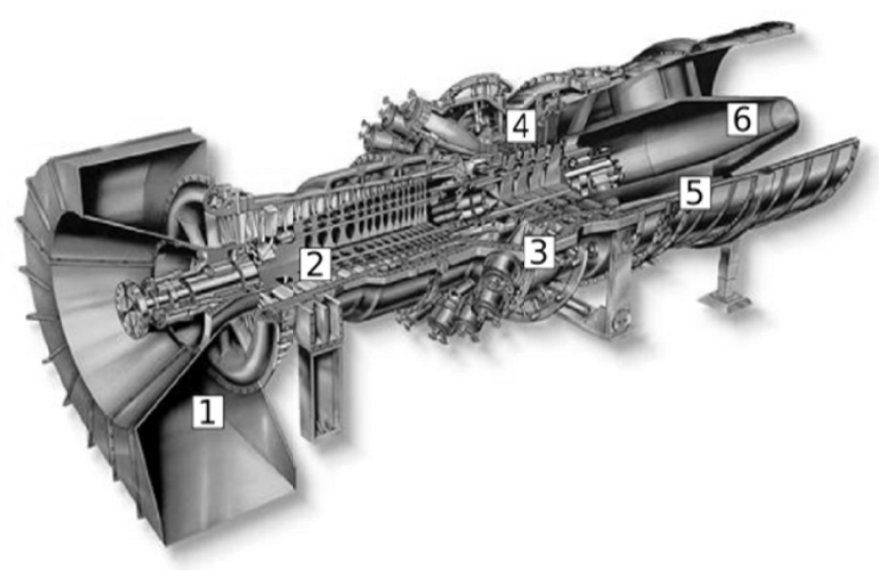

Figure 1-1. Gas turbine engine, Siemens SGT5-4000F. 1 - inlet, 2 - compressor stage, 3 combustion chamber, 4 -turbine, 5 and 6 -exhaust.

\subsection{THERMO-ACOUSTIC INSTABILITIES}

The main drawback of lean premixed combustion of natural gas is its high sensitivity on thermo-acoustic instabilities. Inside the combustion chamber interaction between four phenomena, namely combustion, acoustics, aerodynamics and vibration of the combustion chamber walls take place. Each flame has intrinsic instabilities which lead to noise generation. Two types of noise generated by flames can be distinguished: the autonomous noise which comes from the instabilities in the turbulent flow field in the flame only and driven noise which is a result of mutual interaction between flame aerodynamics and acoustic waves inside the combustion chamber. The latter is of great importance in design and operation of gas turbine engines as it can lead to heavy thermo-acoustic instabilities and subsequently to damage of the combustion system. The unsteady heat release by the flame is an acoustic source and induces pressure waves in the acoustic resonator. These acoustic waves travel downstream of the chamber and after reflection from the boundaries return to the flame. As a result of the impinging acoustic wave, acoustic velocities are perturbing the burner flows. This way the instabilities within the flame are enhanced and the flame fluctuates even stronger. The unsteady combustion process produces high amplitude acoustic waves which increase vibrations of the liner, see Figure 1-2. This behaviour can lead to self-excited oscillations of such high amplitude that fatigue damage of the structural parts is caused. 


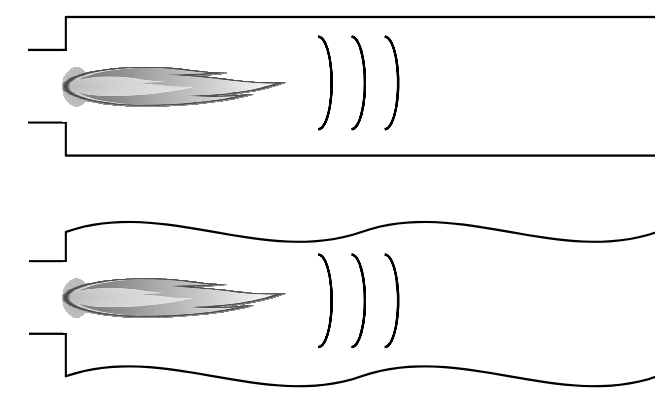

Figure 1-2: Thermo-acoustic instabilities born process

The relation between a flame and the acoustic field was described first by Lord Rayleigh. He stated that at the moment when heat fluctuations released by the flame are in phase with the pressure fluctuations, i.e. when the phase difference lies between $-90^{\circ}$ and $+90^{\circ}$, the thermo-acoustic instabilities are enhanced. On the other hand, the instabilities are damped when heat and pressure oscillations are out of phase, as presented in (Rayleigh, 1878). This is known as a Rayleigh's criterion, see Equation 1-1.

It was observed by other authors, see e.g. (Polifke, Poncet, Paschereit, \& Doebbeling, 2001), (Nicoud \& Poinsot, 2005), (Poinsot \& Veynante, 2005) that Rayleigh's criterion is a necessary but not a sufficient condition for the instabilities to occur. Chu, see (Chu, 1964) introduced changes in the original criterion by taking into account energy losses on the domain boundaries. According to Equation 1-2 the self-exciting instability loop is growing up in amplitude till the saturation limit when the heat released by the flame and pressure fluctuations are in phase and the energy gain exceeds energy losses on the wall.

$$
\begin{gathered}
\int_{\mathrm{V}} \int_{0}^{\tau} p^{\prime} Q^{\prime} d t d V>0 \\
\frac{(\gamma-1)}{\gamma p_{0}} \int_{\mathrm{V}} \int_{0}^{\tau} p^{\prime} Q^{\prime} d t d V>\int_{A} \int_{0}^{\tau} p^{\prime} \boldsymbol{u} d t d A
\end{gathered}
$$

A classic example of the thermo-acoustic phenomenon is a Rijke tube, presented in (Rijke, 1859). It is a vertical, open ended tube with metal gauze placed inside, see Figure 1-3. The gauze is heated up electrically or by flame. Depending on the position 
of the heated grid in the tube, thus on the phase delay between pressure and heat fluctuations, a loud sound is produced. Detailed analysis of the Rijke tube is presented in (Rijke, 1859), (Heckl, 1988), (Hirschberg, 2004). More about thermo-acoustic instabilities in gas turbine combustors can be found in Chapter 3.

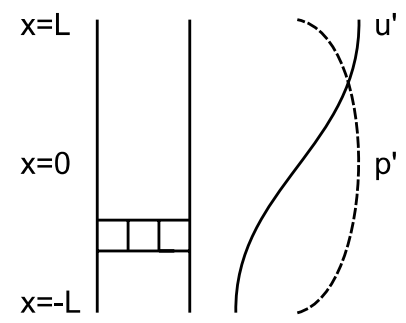

Figure 1-3: Rijke tube

\subsection{Instabilities In The Combustion System}

The life time of a typical gas turbine is mostly limited by thermal and mechanical loads on the turbine blades, and on the combustion chamber liner, see (Tinga, Kampen, Jager, \& Kok, 2007). During lean premixed combustion, the turbulent flame with its enormous thermal power amplifies acoustic pressure changes inside the combustion chamber, as presented in (Ducruix, Schuller, Durox, \& Candel, 2003) and (Lieuwen, Torres, Johnson, \& Zinn, 2001). The combustion chamber is acoustically closed, only a minor part of the sound is able to leave it together with the exhaust gases. Since dissipation of the acoustic wave in the chamber is not significant, most of the sound is reflected from the walls and radiated into the flame, which is highly sensitive on acoustic perturbations. This sensitivity is inversely proportional to the equivalence ratio, i.e. if the combustible mixture is leaner, the flame suffers more of any perturbations in the acoustic field. The acoustic waves propagate upstream to the flame after reflection from the combustor exit. The acoustic source is influenced by the reflected waves and it may start to produce even stronger pressure fluctuations inside the combustion chamber. This behaviour may lead to a higher vibration amplitude of the liner and finally to even stronger changes in the reflected waves and flame itself. Of crucial importance for the operation of the engine is not the noise emitted but its structural integrity. This may be at hazard when the combustor liner starts to vibrate in a mode linked to the thermo-acoustic 
noise. This is even more likely when the combustion noise changes to an unstable closed loop feed-back system. Another dangerous situation may arise when there is a two way interaction between the combustion oscillations and the liner vibration. This strong coupling of the acoustic fluctuations and liner resonance modes induces large amplitude vibrations of the surrounding liner structure. The liner is a critical component because it has to operate reliable at extremely high temperatures. This has significant negative influence on the mechanical strength of the liner. These two factors i.e. high amplitude of vibrations and reduced strength, connected with long operational time, lead finally to fatigue damage of the liner, see (Tinga, Kampen, Jager, \& Kok, 2007) and (Breard, Sayma, Vahdati, \& Imregun, 2002). Examples of fatigue crack in the liner structure are depicted in Figure 1-4. The thermo-acoustic instabilities may also be the source of other undesirable effects occurring in the combustion chamber during combustion, e.g. enhanced heat transfer to the walls, flashback or blow off.

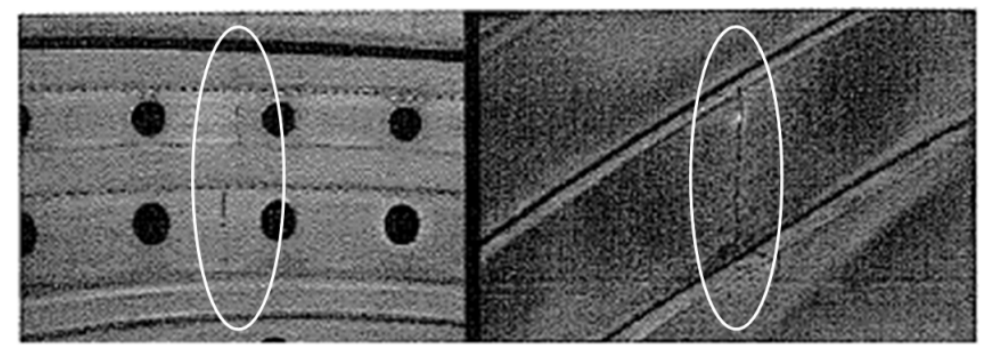

Figure 1-4: Fatigue cracks in the liner structure; from (Tinga, Kampen, Jager, \& Kok, 2007)

The origin of combustion instabilities is well known and it is referred to in many publications: e.g. (Lieuwen, 2003), (Cho \& Lieuwen, 2005), (Hubbard \& Dowling, 2001). However, the fundamental mechanisms of the instabilities are difficult to recognize precisely. Oscillations in heat release or pressure fluctuations are produced by countless mechanisms and physical processes which influence each other, thus the exact recognition of the source is in many cases impossible. Fluctuations in equivalence ratio, unsteady strain rate, interactions between flame and vortex or flameboundaries interaction are a few of them, see (Ducruix, Schuller, Durox, \& Candel, 2003). Also each of the above mentioned processes depends on additional factors. Nozzle geometry, fuel kinetics, heating value, ambient temperature and swirl conditions can cause equivalence ratio fluctuations as described in (Cho \& Lieuwen, 
2005). These processes generate perturbations in heat release ratio by inducing oscillations in flame position or distortion of the enveloping area of the flame.

Two basic procedures of removing thermo-acoustic instabilities can be recognized. In the passive mechanism, the instabilities are damped by changing the gas turbine geometry, flow conditions and acoustic damping of the chamber, as presented in (Putnam, 1971). This could be done by breaking the symmetry in the gas turbine, adding a pilot flame or introducing quarter wave tubes or Helmholtz resonators. In the latter case, the resonators present the best performance when they are tuned to the frequency of the instabilities. Since the equivalence ratio, temperature, flow velocity etc. have direct influence on the instabilities, the bandwidth of the resonators must be broad enough to capture these variations. However, a broader frequency bandwidth entails decreasing efficiency of acoustic damping, see (Bellucci, Rohr, Paschereit, \& Magni, 2004). Generally, the passive damping methods have application in some specific conditions. Any changes in the system characteristic or operating conditions may affect the already tuned gas turbine performance and destroyed the desired effect.

Another procedure is the active damping of thermo-acoustic instabilities by a closed feedback loop mechanism which monitors and suppresses unsteadiness in the combustion chamber, see (Ziada \& Graf, 1998), (Dowling \& Morgans, 2005). This system checks the amplitude and frequency of the instabilities and then sends back a compensating signal which disturbs the interaction between heat oscillations and acoustic pressure pulsations. As a consequence the thermo-acoustic instabilities are damped. Since the gas turbine operates in a wide spectrum of operating conditions and the source of the instabilities might differ for various operating points, the active damping controllers must be robust enough to capture and suppress all instabilities. On the other hand, increasing robustness of the controllers has negative influence on the error introduced to the compensating signal. Therefore, the gas turbine manufactures equip the gas turbine engines with active control systems reluctantly.

\subsection{VIBRATIONS}

Vibrations are unavoidable phenomena in any working machine. Gas turbines are not an exception. Vibrations in a typical combustion chamber of a gas turbine are directly related to pressure changes induced by the flow and fluctuation in heat release by the flame. When thermo-acoustic instabilities are not present in the system, the amplitude of vibration is relatively small and the turbine can work for a desired long 
time without any maintenance. However, in case self-excited instabilities arise in the system, especially when their frequency is nearby, or even match with the resonant frequency of the combustion chamber walls, the life time of the chamber can be reduced significantly. A fluctuating acoustic pressure in close proximity to the liner walls generates acoustic loads on the surface. Even when the medium is air, acoustic loads, exerted with the frequency matching the resonant frequency of the liner, can lead to high vibration amplitude. A high amplitude of pressure changes leads to high forces on the liner face that finally induces strong fluctuations in the material. Vibrating walls are another acoustics source. They emit acoustic waves to the surroundings, as it is presented in (Kaczor \& Sygulski, 2005). In case of gas turbines, additional pressure waves can be obtained inside the combustion chamber and in the cooling passage where the cooling medium is flowing.

Not all the acoustic energy is absorbed by mechanical vibrations. A part of the energy is dissipated during the walls movement. Generally, two main damping mechanisms can be distinguished, namely energy dissipation as a result of surface effects, arising during the relative wall movement, and energy dissipation as an internal effect during a body deformation, see (Engel, 2001). Those two mechanisms can be divided further depending on specifics of the investigated problem. Vibrations of the liner in the combustion chamber are mainly limited by:

- Material damping. Damping of the vibration energy in the metallic structure itself. The wave energy inside the body is converted into heat. Material damping increases with the distance from the point of excitation.

- Construction damping. It depends on boundary conditions used for the support of the vibrating structure. This kind of damping is observed during deformation of material (an internal friction damping) when a relative displacement between the body molecules take place.

- Aero-dynamical damping. In this case damping comes from an external resistance of the movement, i.e. an air resistance inside the gas turbine flow channels.

\subsection{RESEARCH OBJECTIVE}

The underlying work is motivated by the recent need for leaner combustion technologies and reduced emissions which led to appearance of the combustion instabilities in many combustion systems. In general, the problem of fluid-structure 
coupling is not limited to stationary gas turbines for power production, but applies to any combustion system such as aero engines or rocket boosters. The research activities in FLUISTCOM are towards improved understanding of transient combustion and its coupling with combustor wall vibration. The objective is to come to well-validated models of transient combustion and wall vibration. Both experimental measurements and numerical calculations are carried out to determine the correlation between acoustic pressure oscillations on one side and liner vibrations on the other side. New interfaces between numerical combustion codes, thermo-acoustic codes on one hand and Finite Element codes for mechanical stress and solid vibrations on the other hand will be developed. The integrated/coupled analysis is a prerequisite for a stable/robust structural mechanical design. This can lead to design rules of combustors that are extremely robust even in combustion oscillatory situations. Research especially on the correlation between vibration frequencies and amplitudes of the structure and the acoustic pressure fluctuations are new in the field of combustor design. In particular issues related to interaction of the combustion system with the liner structure are not much understood.

\subsection{OUtLine}

The work presented in this thesis is divided into five chapters. All chapters are correlated with each other according to Figure 1-5.

In Chapter 2, the measurements performed in the combustion test rig are presented. First, the detailed configuration of the laboratory scale test rig together with the location of data acquisition points and operating conditions under investigation is presented. Then the results of the experiment conducted on steady and pulsating flow conditions, followed by flame transfer function investigation are discussed.

Chapter 3 is devoted to the CFD modelling of the combustible flow. Different turbulence models are used for the static and dynamic analysis of the combustion system. The influence of time step and heat transfer coefficient on the obtained results is shown. The results of the numerical approach are compared with the pressure, temperature and chemiluminescence data recorded inside the combustion chamber during the combustion experiment.

In Chapter 4 fluid-structure interaction computations (FSI) between flue gas and liner, with the use of a partitioned approach are presented. The Ansys-CFX code for the CFD computations is combined with the Ansys Multiphysics code for the FEA into one computational process. The exchange of information between the codes is done through 
interface connection, created by a coupling code, every time step. Two different approaches, namely one-way and two-way interaction, are considered. The results of the FSI computations are compared with the experimental signals of pressure and velocity fluctuations.

The acousto-elastic and modal analysis are presented in Chapter 5. For both the FEM is used. The acousto-elastic analysis uses the pressure field obtained from the CFD computations (presented in Chapter 3) as input conditions for further investigation of mutual interaction between the acoustic field and walls vibrations. In the modal analysis the hazardous acoustic and coupled eigenfrequencies of the combustion chamber are marked. Similarly to the previous chapters, all numerical data are compared with the experimental results.

In Chapter 6, the backward facing step test case with heat transfer across a wall is taken under investigation. Due to its simple geometry and the availability of well documented experiments the backward facing step with heat transfer represents an interesting validation case. Results of steady-state calculations with the use of various turbulence models are compared here with the available literature data. Moreover transient calculations with the use of pulsating inlet velocity and oscillating heated wall are presented.

In the last chapter conclusions and recommendations for further research are presented. 


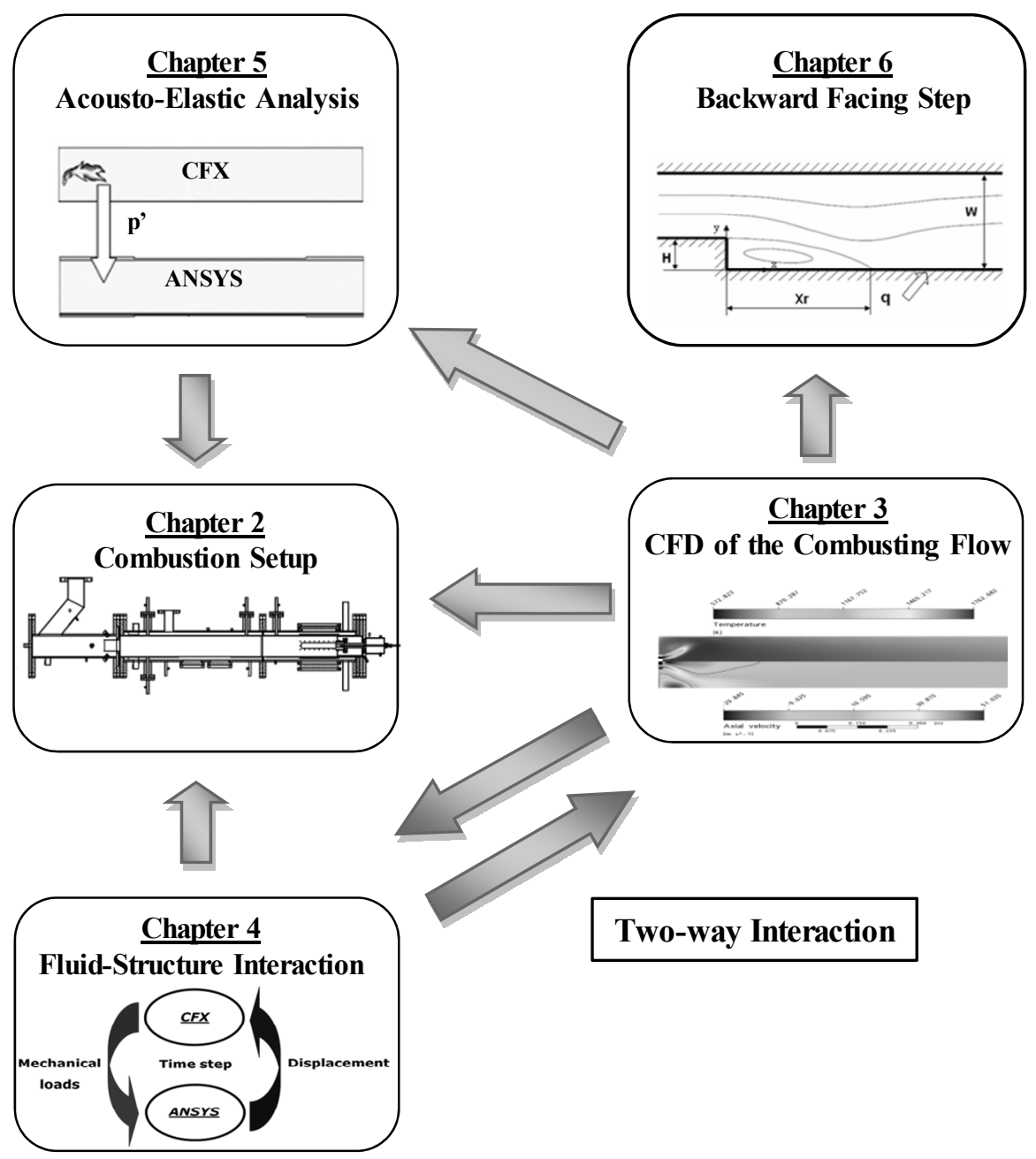

Figure 1-5: Relations between chapters presented in the thesis 



\section{Combustion SetuP}

\subsection{INTRODUCTION}

This chapter presents the combustion test rig available in the Laboratory of Thermal Engineering at the University of Twente. The main geometry together with the location of data recording points and operating conditions under investigation are described first. Then the results of the experimental investigation with the use of two different liner configurations and various operating points are discussed. All experiments are performed at steady and at pulsating flow conditions. Data obtained during the investigation serve for reconstruction of the flame transfer function, validation of the numerical results and investigation of the wall flexibility and absolute pressure level effect on the combustion dynamics.

\subsection{BACKGROUND}

The combustion setup under investigation (main part of the setup is presented in Figure 2-1) is intended to be representative for a section of a full scale gas turbine annular combustion chamber. Due to significant geometrical dimensions and high operational costs it is unpractical to build the whole combustion chamber together with its 24 burners. Instead, a slice of the chamber with only one burner and with reduced thermal power is manufactured. In order to obtain similar acoustical and structural eigenfrequencies as during operation on the full scale device, the geometrical dimensions of the investigated setup are matched in scale. As a consequence of the setup resizing, the flame occupies only the top part of the combustion chamber in the vicinity of the burner mouth, whereas in the industrial gas turbine, the flame occupies a major part of the combustion chamber. The test rig can work with a maximum thermal power equal to $500 \mathrm{~kW}$ at 5 bar absolute pressure. The investigated flame is a natural gas lean premixed preheated flame. Stabilization of the flame is done by inner and outer recirculation regions created by a swirler and sudden 
expansion of the geometry profile. To decrease the overall temperature of the structural parts exposed to the flame and hot gases, between the liner and pressure vessel a cooling flow is present. Several pressure transducers and thermocouples are located along the setup to monitor temperature and pressure at various locations. Data from the temperature, pressure and flow sensors is collected by LabVIEW and SigLab hardware and software. Further data examination is done with the use of Matlab 7.1 environment. Technical information about these products can be found in (National Instrument, 2003), (DSPSigLab, 2001) and (The MathWorks, 2005), respectively.

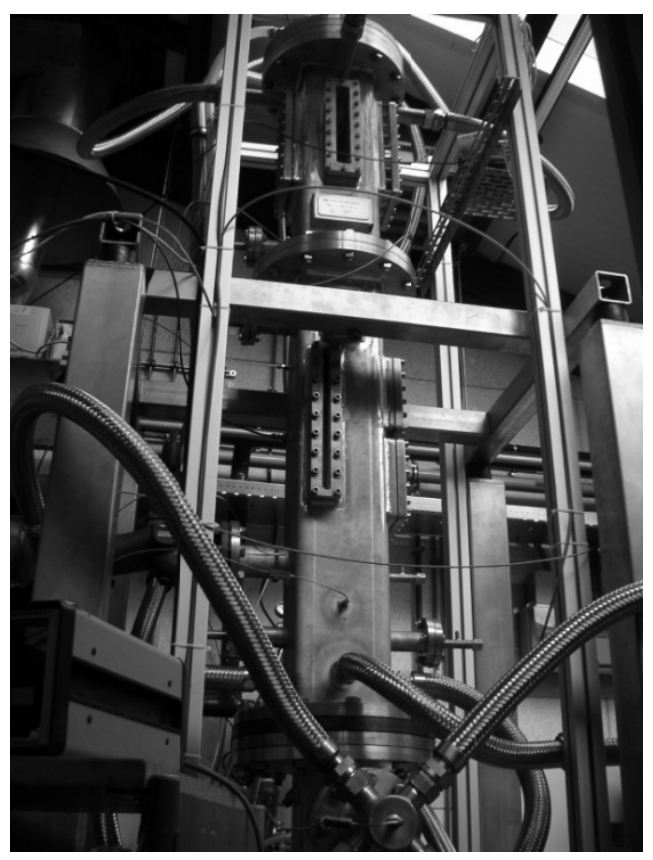

Figure 2-1: Combustion test rig

The combustion test rig has a modular built. This assures that the individual parts of the setup can be easily disassembled and replaced by new ones, which fit better to the type of research that is conducted at any given time. The cross-section of the combustion test rig together with its modular parts and location of data recording points is presented in Figure 2-2, where $\mathrm{P}$ and $\mathrm{Ps}$ are dynamic and static pressure sensors respectively, $\mathrm{T}$ are thermocouples, LDV is Laser Doppler Vibrometer and CCD stands for the camera for chemiluminescence measurements. 


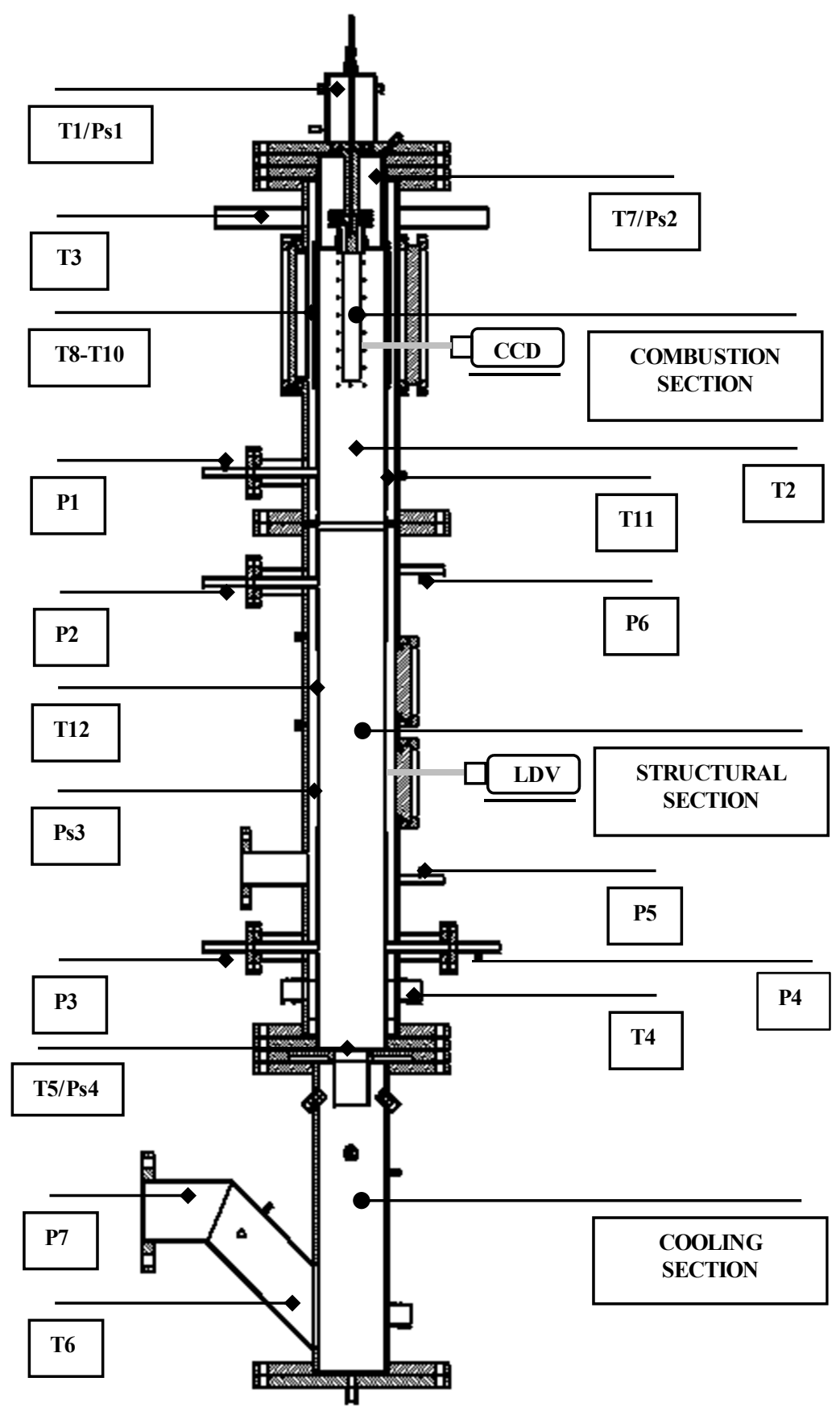

Figure 2-2: Combustion setup configuration 
The main modular parts of the combustion test rig are the combustion section, structural section and cooling section. Since the configuration of the structural section has a major impact on the liner vibration characteristics, this section is described in details later in this chapter. First, the other two sections are shortly presented below.

The combustion section is located just behind the burner mouth. In this section combustion process takes place. First the combustible fuel-air mixture is ignited by a spark plug. Then, the flame is self sustained and stabilized by recirculation regions of hot gas which ignites the fresh mixture. To make observations of the flame the setup is equipped with a system of windows. The windows are mounted in the liner and pressure vessel. Chemiluminescence and Planer Laser Induced Fluorescence (PLIF) are used to gather information about the flame composition. Additional information about measurements with the use of chemiluminescence and PLIF with the setup presented in this thesis can be found in Chapter 3 and in (Harleman, 2005) and (Van Kampen, 2006).

The main task of the cooling section is to reduce the temperature of the exhaust gases. In order to do so, the hot flue gases are mixed first with the cool air from the cooling passage and then to decrease their temperature even more, they are sprayed with cold water. Therefore, the gases which leave the setup are cooled down sufficiently to pass the throttle valve and chimney, and finally they can be released to the atmosphere. The second task of the cooling section is to provide a uniform pressure distribution on either sides of the combustion chamber and cooling passage. In account of the small thickness of the liner, the absolute pressure in the combustion chamber must be equal to the pressure inside the cooling passage. Otherwise, high mechanical stresses can occur in the liner walls and finally they might lead to the liner damage. To equalize pressure on both sides of the liner, i.e. in the combustion chamber and cooling passage, the latter is connected with the cooling section by four steel bypass hoses. Since the cooling section is also connected with the combustion chamber, only minor pressure differences in the combustion chamber and cooling passage are possible. Furthermore, at the place where the structural section is connected with the cooling section, a sudden contraction of the combustion chamber exists, see Figure 2-2. This contraction combined with the water spray in the cooling section forced most of the acoustic waves to reflect back inside the combustion chamber and provides at the same time a well defined acoustic boundary condition for further numerical analysis. 


\subsection{Structural Section}

The structural section is the part of the combustion test rig where the vibrations of the liner are measured. To make the liner more sensitive to the pressure changes inside the combustion chamber, part of it has a smaller thickness with comparison to the overall wall thickness. Thus any variations in the pressure pattern inside the combustion test rig are immediately translated to changes in the vibration amplitude and frequency of the flexible section. Furthermore, the flexible section located between much more stiffer and thicker liner parts has well defined structural boundary conditions for the numerical analysis of the structural vibrations.

In order to obtain information about the liner vibrations amplitude and frequency, the flexible section must be able to vibrate freely without any measurement induced damping. Therefore, a non-invasive technique is used for vibration data collection and all thermocouples and pressure transducers are placed at some distance from the flexible section. Since the Laser Doppler Vibrometer is the technique employed for vibration data collection, it is necessary to have access to the vibrating liner via transparent windows. Two different shapes of windows are used. Square windows are employed to obtain a twodimensional pattern of the liner vibrations, whereas to observe the longitudinal modes only, slit windows are enough. For the liner configuration presented here, the structural modes in the investigated frequency range show mostly the one-dimensional shape. Moreover, the experimental time spent to obtain good two-dimensional data from the square windows is an order of magnitude longer then the time spent for 1D data. Therefore, to reduce recording time, data presented in this thesis are for 1D measurements of the liner vibrations through the slit window.

Two liner configurations with different thickness and length of the flexible section are investigated subsequently. The overall size of both is the same, 150/150/1813 mm and 4 $\mathrm{mm}$ thickness of the stiff part. The difference lies in the dimensions of the flexible part. In the configuration called Desire, the flexible section thickness is equal to $1.5 \mathrm{~mm}$ and has a length of $400 \mathrm{~mm}$, whereas in the Fluistcom configuration, the flexible part has a thickness equal to $1 \mathrm{~mm}$ and length equal to $680 \mathrm{~mm}$. The width is always $150 \mathrm{~mm}$. Both names i.e. Desire and Fluistcom come from the names of the European projects during which the liners were manufactured. The Desire liner configuration represents the stiff liner configuration due to smaller and thicker flexible section, while the Fluistcom - the flexible one. The influence of liner configuration on thermo-acoustic instabilities is investigated during the combustion tests at various operating conditions. 
The entire liner consists of two main parts: a top and a bottom one. The top part with a window for optical access to the flame belongs to the combustion section. The bottom part of the liner with flexible walls is associated with the structural section, see Figure 2-3.

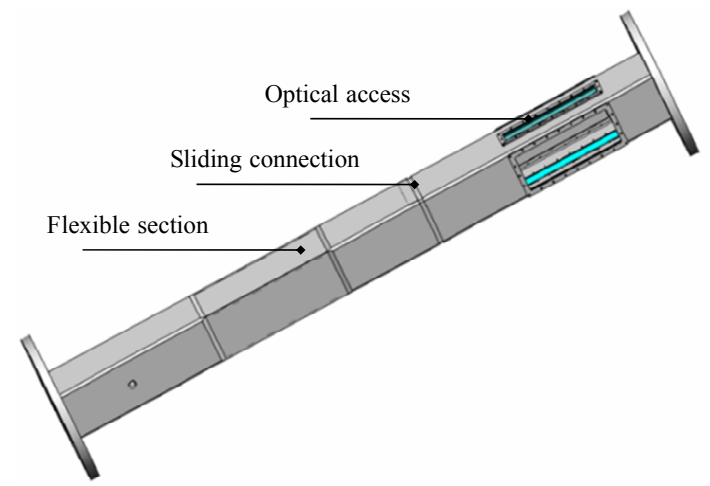

Figure 2-3: Liner configuration (here the Desire liner is presented)

Both liner parts are joined by a sliding connection, see Figure 2-4. The connection allows free expansion of the liner walls in the longitudinal direction due to temperature loads. Since the liner can expand freely in the longitudinal direction and has small thickness, only minor thermal stresses due to work at high temperature are expected.

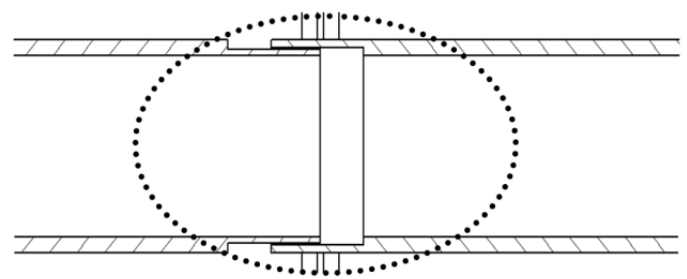

Figure 2-4: Cross-section of the sliding connection between liner parts (for compactness in horizontal position)

Numerical computations of the thermal stresses inside the combustion chamber walls presented in Appendix C, confirm that their influence on the liner in current configurations is minor. Investigations of different liners with various shape, thickness and length of the flexible section with regard to eigenfrequencies are presented in Appendix B. A comparison 
of the eigenfrequencies obtained for the clamped vibrating plate in analytical and numerical way is presented in Appendix A.

\subsection{LASER DOPPLER VIBROMETER}

Measurements of the liner vibrations are performed with the use of a Laser Doppler Vibrometer. The principle of this measurement technique is presented in Figure 2-5. The laser beam is divided by a splitter in two beams. The reference beam stays inside the laser device and the measurement beam is pointed to the vibrating object. The measurement beam is scattered by the vibrating body with a frequency shift proportional to the instantaneous velocity of the object. Based on the comparison between reference and measurement beams, the velocity of the vibrating body is determined. The main advantages of the Laser Doppler Vibrometer are high accuracy and that no physical contact with the vibrating surface is required. This allows to measure the velocity of a very hot surface without introduction of added resistance to the vibrating wall. The drawback of this technique is high sensitivity of the laser on the reflectivity changes in the measured surface due to variations in temperature. Additionally there is an influence of the elevated temperature in the room where the LDV is placed on the signal quality. A detailed description of the Laser Doppler Vibrometer can be found in (Polytec GmbH, 2003). Application of LDV in combustion devices is presented in (Paone \& Revel, 2000) and (Gasparetti, Paone, \& Tomasini, 1996).

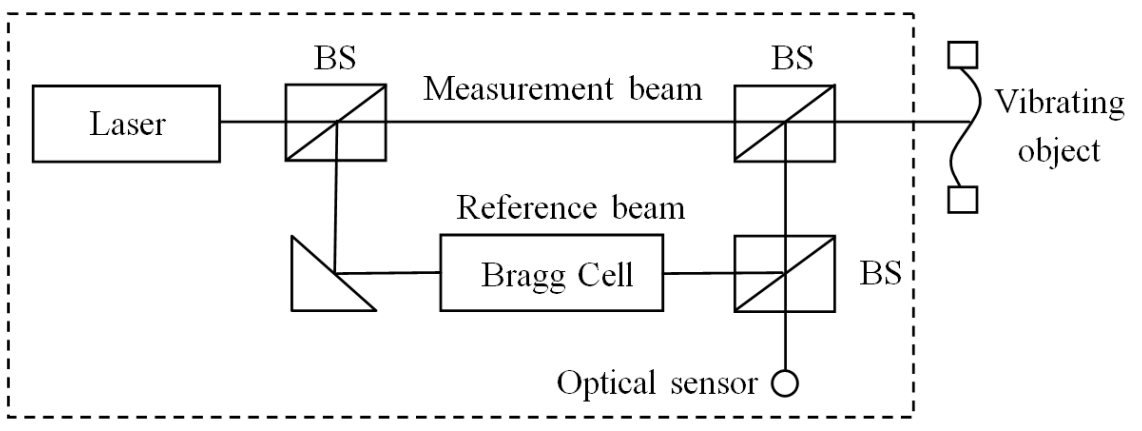

Figure 2-5: Scheme of the Laser Doppler Vibrometer 
Vibration measurements are done through a slit window located at $25 \mathrm{~mm}$ from the edge of the liner. In the vertical direction measurements are performed at one point in the middle of the flexible liner section, see Figure 2-6.

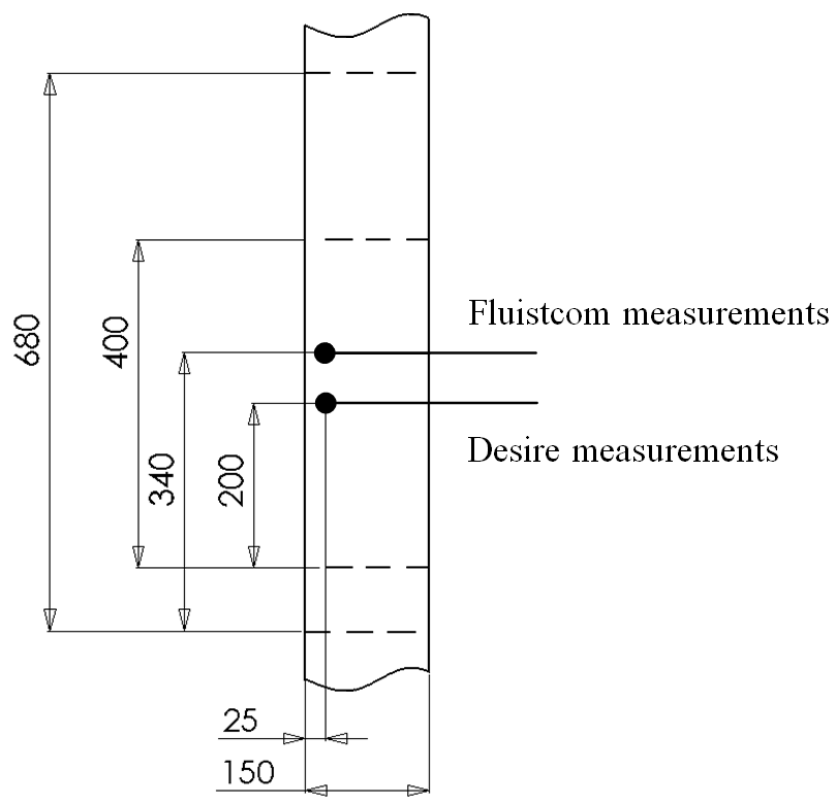

Figure 2-6: Location of the vibration measurements

\subsection{Pressure Sensors}

Temperature and pressure signals are collected at different locations along the setup see Figure 2-2. To leave the liner vibration patter undisturbed, thermocouples and pressure transducers are located at some distance from the flexible section. That is why comparison of the liner vibrations and acoustic pressure is done at different positions. To record the pressure signal, the Kulite pressure sensors are employed. These measure the acoustic pressure range between 0 bar and 0.35 bar. Since temperature above $175^{\circ} \mathrm{C}$ can destroy the diaphragm placed inside the sensors, to decrease the risk of damage, the sensors are mounted on tubes located on the combustion casing, as is presented in Figure 2-7. In order to decrease the temperature influence even more, the Kulite sensors are cooled down by pressurized air delivered by a system of small pipes. 
The side tubes are connected to semi-infinite hoses with a closed end. This configuration has two main advantages. First, temperature influence is even more decreased because the hot gases from the combustion chamber are hardly convected to the pressure sensors. Second, due to filling the end of the hose with a porous material, the incoming acoustic wave is absorbed. This assures that the measured acoustic wave has a source in the investigated chamber and it is not an effect of a resonance inside the side tube or hose.

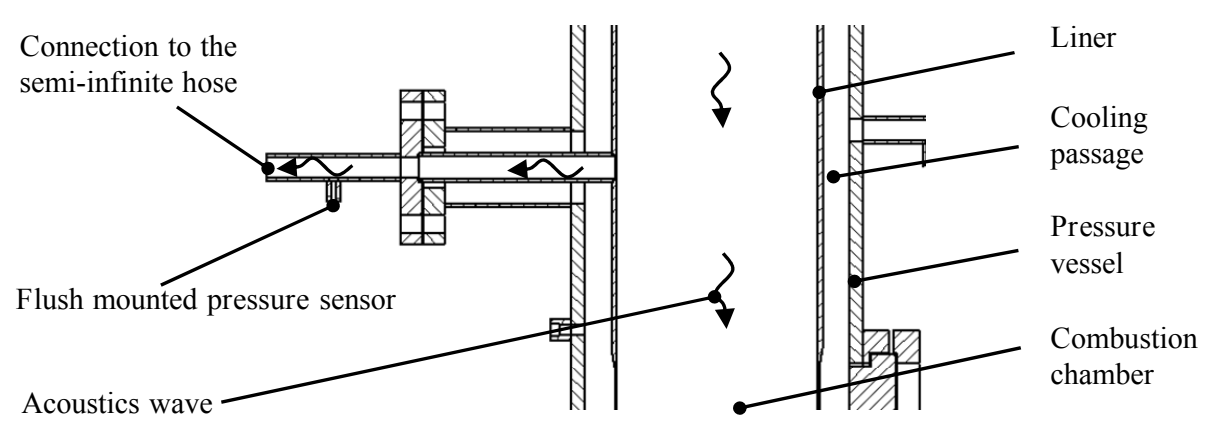

Figure 2-7: Connection of the pressure transducers

For the experiments conducted at an elevated absolute pressure, the static pressure should be equalized on both sides of the sensor diaphragm in order to prevent the diaphragm burst, which can occur when the pressure difference exceeds 1.05 bar. Therefore, the rear inlet of the pressure transducer is connected with a long and thin hose to an expansion volume, which is also connected to the combustion chamber. Due to viscothermal losses inside the hose and introduction of acoustic volume, the acoustic energy transmitted from the combustion chamber to the rear inlet of the sensor is reduced significantly. More information about measurements with the use of Kulite sensors are presented in (Huls, 2006), (Van Kampen, 2006), (Pater, 2007). Specification of Kulite pressure transducers can be found in (Kulite Semiconductor Products Inc, 2008).

\subsection{Operating Points}

The lean premixed natural gas flame is investigated under various conditions. The investigation range covers thermal powers between $100 \mathrm{~kW}$ and $250 \mathrm{~kW}$ and absolute pressures of $1-3$ bar. For simplicity, the investigated conditions are characterized with a 
code number for the operating points. Three operating points are presented in Table 2-1. All of them are performed for steady and oscillating flow. For the latter, the equivalence ratio is oscillated with prescribed frequency and amplitude leading to active perturbation of the flame. The equivalence ratio is defined according to Equation 2-1. The experimental results of pulsating flow are used for validation of the numerical investigation.

\begin{tabular}{|c|c|c|c|c|c|c|}
\hline $\begin{array}{c}\text { Operating } \\
\text { point }\end{array}$ & $\begin{array}{c}\text { Thermal } \\
\text { power } \\
{[\mathbf{k W}]}\end{array}$ & $\begin{array}{c}\text { Absolute } \\
\text { pressure } \\
\text { [bar] }\end{array}$ & $\begin{array}{c}\text { Thermal } \\
\text { power/pressure } \\
\text { ratio }[\mathrm{kW} / \mathrm{bar}]\end{array}$ & $\begin{array}{c}\text { Air } \\
\text { factor } \\
{[\mathrm{K}]}\end{array}$ & $\begin{array}{c}\text { Mass flow } \\
\text { rate }[\mathrm{g} / \mathrm{s}]\end{array}$ & $\begin{array}{c}\text { Preheating } \\
\text { temperature } \\
{[\mathrm{K}]}\end{array}$ \\
\hline 15.7 & 125 & 1.5 & 83 & $\overline{1.8}$ & 75.5 & 300 \\
\hline 20.8 & 187 & 2.3 & 81 & 1.8 & 113.0 & 300 \\
\hline 30.5 & 250 & 3.0 & 83 & 1.8 & 151.1 & 300 \\
\hline \multicolumn{7}{|c|}{ Table 2-1: Investigated operation points } \\
\hline & & $\phi_{0}=$ & $\frac{{ }_{, 0} / y_{a, 0}}{\left.{ }_{0} / y_{a, 0}\right]_{s t}}$ & & & $2-1$ \\
\hline
\end{tabular}

In the equation, $\phi_{0}$ is the mean equivalence ratio, $y$ is the mean mass flow and subscripts $f$, $a$, st stands for fuel, air and stoichiometric, respectively. The stoichiometric ratio is an exact amount of air to burn fuel completely. In this thesis the term air factor, which is the reciprocal of the equivalence ratio is also used.

\subsection{Steady and Pulsating Measurements}

During the experiment, the pressure and velocity signals are recorded with the use of Siglab hardware and software in a frequency range up to $2 \mathrm{kHz}$. For both, steady and pulsating flow measurements, the total number of 8192 samples per bandwidth is taken, which gives the frequency resolution equal to $0.24 \mathrm{~Hz}$. In order to eliminate randomness in the recorded signal, data in the frequency domain are averaged over 20 measurements. Due to restrictions given by Siglab, in the time domain only the last measurement is saved, see (DSP-SigLab, 2001).

For measurements with pulsating equivalence ratio a MOOG valve is used. The MOOG valve perturbs the mass flow of the fuel in a controlled way. The piston built in the device 
is opening and closing fuel channel according to the prescribed signal. The position of the piston is monitored by a displacement sensor. The frequency and amplitude of the pulsation is controlled by the Siglab device which sends the signal in the form of voltage change to the MOOG valve. Detailed technical specification of the MOOG valve D633-7320 can be found in (Moog Inc, 2008). The equivalence ratio fluctuations controlled by the MOOG valve in the present combustion test rig can be changed in the frequency range up to 400 Hz. The maximal amplitude equals 10\%, see (Van Kampen, 2006) and (Kleinlugtenbelt, 2005). Measurements with pulsating frequency beyond $400 \mathrm{~Hz}$ are possible but the available level of excitation decreases rapidly with increasing frequency.

To obtain data for validation the numerical results, the equivalence ratio controlled by the MOOG valve is oscillated with frequency of $300 \mathrm{~Hz}$ and amplitude equal to $8.5 \%$ of the mean equivalence ratio. Experimental data is evaluated for the velocity signal in the middle of the flexible section (in vertical position, see Figure 2-6) and for the pressure signal at position of pressure transducers P1, P2, P3, P5 and P6. Location of the pressure transducers is depicted in Figure 2-2. The results show the signal spectrum in time and frequency domain. Two liner configurations are investigated: Fluistcom which represents the flexible liner and Desire for the stiff liner. The experimental results are evaluated for the operating point 15.7, see Table 2-1.

\subsubsection{STEADY Flow RESUltS}

Experimental results obtained during steady flow measurements for the Desire and Fluistcom liner are investigated here. The results are evaluated for the velocity and pressure data first. Then, the acoustic eigenfrequencies of the system are presented.

Typical results of the measured wall velocity and the pressure at locations P1, P2, P3, P5 and P6 in the spectral domain are shown in Figure 2-8. All data show a random spectrum with a self excited frequency peak just above $400 \mathrm{~Hz}$. The location depends on the operating conditions. For the Desire liner configuration the thermo-acoustic instabilities appear at $454 \mathrm{~Hz}$, for the Fluistcom at $429 \mathrm{~Hz}$. The difference in the measured frequency of the instabilities is due to a slightly different temperature profile inside the combustion chamber and in the cooling passage, observed during both experiments. The changes in the temperature appear due to variations in the cooling air to combustion air ratio, which during the various experiments was set in the range between 1 and 1.2. Also the liner geometry has a twofold influence on the main instabilities. A direct influence due to a different vibration pattern and thus different feedback to the pressure field and an indirect influence by changing the temperature field inside the chamber by affecting the heat transfer through the 
walls. The deviations in the location of the main instabilities are not only caused by a different liner geometry, but they are also present within the same configuration. In case of the experiments with the use of the Desire geometry, the self-excited oscillations are observed in the frequency range $433 \mathrm{~Hz}-458 \mathrm{~Hz}$ whereas for the Fluistcom experiment, the instabilities are located between $405 \mathrm{~Hz}$ and $431 \mathrm{~Hz}$. The maximum amplitude of the instabilities also varies within the investigated cases. The thermo-acoustic instabilities do not appear immediately after the operating point is reached, but they need some time to grow up to the saturation level. Therefore the time spent between the moment when the operating point is reached and the measurements are started, has a significant influence on the instability magnitude. Since during the experiments the saturation level of the instabilities is not investigated, a discrepancy between the starting time of the individual measurements may exist. For the long operational time at the operating point which presents unstable characteristics the amplitude of instabilities can exceed $140 \mathrm{~dB}$. Despite of these discrepancies, the results show that for the more flexible liner configuration the self-excited thermo-acoustic instabilities are moved slightly into the lower frequency region, with comparison to the Desire results.

The recorded velocity signal shows the same trend as the pressure spectrum. The main frequency of vibration is located in the vicinity of the frequency at which the thermoacoustic instabilities occur. The influence of the stiffness of the flexible section manifests itself in the vibration frequency which is shifted to the low frequency range in case of the Fluistcom geometry. Here, next to the main velocity peak at $429 \mathrm{~Hz}$ also the secondary strong peak at $228 \mathrm{~Hz}$ is visible. Around this frequency the first acoustic mode exists (see further part of this section). Due to the high liner flexibility even a weak coupling with an acoustic mode leads finally to the enhancement of the structural and acoustic vibrations. Similarly a coupling between the flexible liner and acoustic modes is visible for higher frequencies. Since the liner in the Desire configuration has a higher stiffness than the one in the Fluistcom, mainly the vibrations at the frequency of the instabilities are visible. 

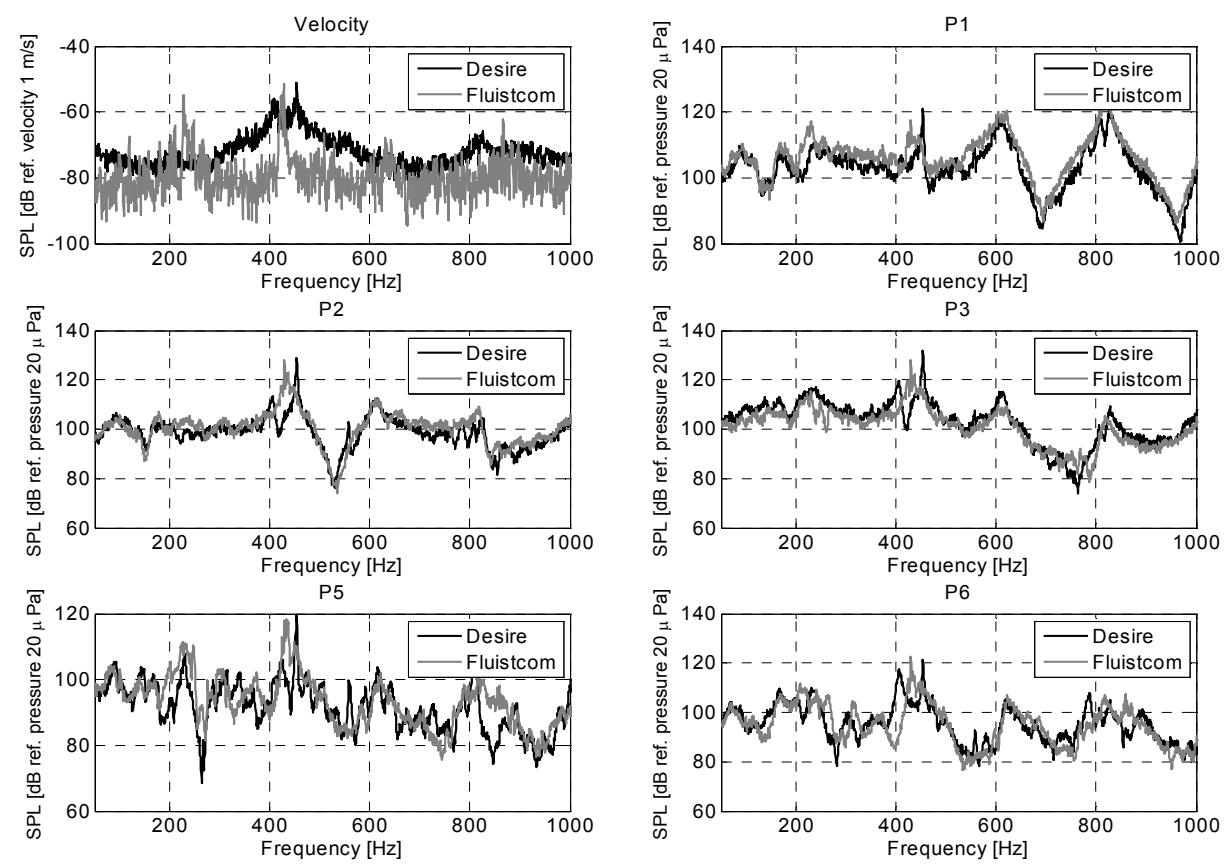

Figure 2-8: Results of the steady flow experiment

In the cooling passage, the pressure transducers P5 and P6 show signal spectra which resemble the pressure signal in the combustion chamber. The influence of the thermoacoustic instabilities is visible. The amplitude of the peak is several $\mathrm{dB}$ lower than in the combustion chamber. Also additional peaks around acoustic eigenfrequencies of the combustion chamber are observed. These might be transmitted by vibrating walls or through the bypass connection between cooling section and cooling passage. The remaining peaks visible on the cooling passage spectrum come from the acoustic eigenfrequencies of the passage combined with the wall vibrations and turbulences induced by flow around the connection for the pressure transducers, thermocouples, bypass connection etc. These obstacles make the signal in the cooling passage more undulating.

Acoustic natural frequencies are obtained from the average spectrum of the all pressure transducers located in the combustion chamber. This way, the influence of acoustic nodes on the spectrum is eliminated. In the investigated frequency range, six acoustic modes are observed. They are presented in Figure 2-9 and Table 2-2. 


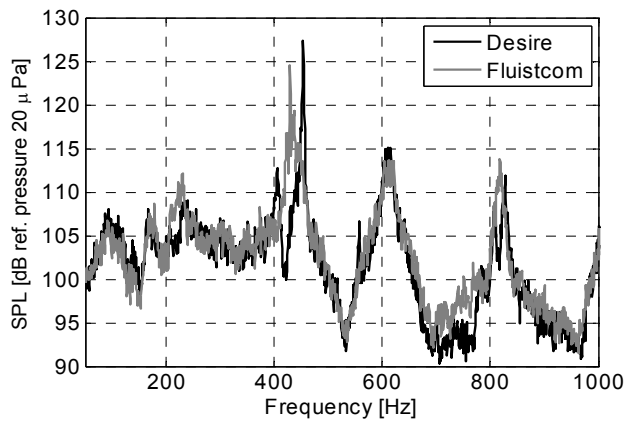

Figure 2-9: Acoustic modes of the setup

\begin{tabular}{ccc}
$\begin{array}{c}\text { Mode } \\
\text { number }\end{array}$ & \multicolumn{2}{c}{ Frequency [Hz] } \\
Desire & Fluistcom \\
\hline- & 93 & 92 \\
- & 166 & 169 \\
1 & 239 & 230 \\
2 & 454 & 429 \\
3 & 610 & 612 \\
4 & 828 & 818
\end{tabular}

Table 2-2: Acoustic eigenfrequencies

Two first modes visible around $92-93 \mathrm{~Hz}$ and $166-169 \mathrm{~Hz}$ are not visible in the mode spectrum of the combustion chamber presented in Chapter 5. These modes are discussed in (Huls, 2006) and (Sengissen, Poinsot, Van Kampen, \& Kok, 2007), they come either from the whole setup or from the cooling section. The remaining four modes represent the first four acoustic eigenfrequencies of the combustion chamber. For most of the modes a minor difference between the Desire and Fluistcom geometry is observed. Only for the second mode a significant percentage difference in the eigenfrequency is found of around $6 \%$. The thermo-acoustic instabilities appear around the second acoustic mode. Therefore they have impact on the frequency shift. Since the frequency of the thermo-acoustic instabilities and the second acoustic eigenfrequency are alike, it is difficult to judge whether the peak visible on the spectrum presented in Figure 2-9 comes from the acoustics only or from the thermoacoustic instabilities and acoustics. In spite of the uncertainties mentioned above, it is observed during the series of experiments with the use of different liner configurations, that the second acoustic mode in case of the Fluistcom configuration is placed generally at slightly lower frequency with comparison to the Desire geometry. Therefore there is some influence of the investigated liners on the acoustic performance of the system. In Chapter 5 acoustic natural frequencies of the combustion chamber computed with the use of the FEM method are presented.

\subsubsection{Pulsating Flow Results}

In this section results of the experiments performed with fluctuating mass flow rates of fuel are presented. Fluctuations are done with a frequency equal to $300 \mathrm{~Hz}$ and amplitude 
of $8.5 \%$ of the mean equivalence ratio (this frequency and amplitude were chosen according to (Van Kampen, 2006)).

The pressure spectra of all pressure transducers present similar behaviour. The forcing peak is located exactly at $300 \mathrm{~Hz}$ and the thermo-acoustic instabilities are placed in the vicinity of the second acoustic mode, see Figure 2-10. Similar to the investigation with steady fuel flow, the Fluistcom liner configuration shows instabilities at a lower frequency with respect to the Desire geometry. The main instabilities are visible at $431 \mathrm{~Hz}$, whereas for the Desire configuration, they are placed at frequency of $439 \mathrm{~Hz}$. At a frequency of $614 \mathrm{~Hz}$ for the Fluistcom and $640 \mathrm{~Hz}$ for the Desire liner configuration the secondary instabilities appear. They are nearby the third acoustic mode located around $612 \mathrm{~Hz}$. This behaviour presents a strong correlation between thermo-acoustic instabilities and acoustic natural frequencies of the combustion chamber. In case of pressure transducer P1, the peaks around the third and fourth acoustic frequency are even more significant than the main instabilities. Behaviour like that is also visible during the measurements without pulsation, see Figure 2-8. This could be an effect of the pressure node located somewhere nearby the location of pressure transducer P1.

The local velocity spectrum shows a coupling between acoustic pressure fluctuations and structural vibrations. The strongest coupling is with the second acoustic mode, where the thermo-acoustic instabilities are visible. The Fluistcom configuration presents coupling with all acoustic modes. However here, the main frequency of the liner vibrations is moved from frequency of $431 \mathrm{~Hz}$ to $417 \mathrm{~Hz}$. This is expected by the modal analysis, discussed in Chapter 5, that shows at a frequency equal to $420 \mathrm{~Hz}$ a structural mode exists which includes not only the flexible part of the liner, but the whole liner. A similar mode is observed for the Desire configuration at a frequency equal to $402 \mathrm{~Hz}$. However in this case the main vibration frequency is not shifted but the velocity peak stays at the thermoacoustic instability frequency.

The signal recorded in the cooling passage presents more unstable behaviour due to the influence of all obstacles mentioned earlier. The main trend of the signal resembles more the pressure observed in the combustion chamber than the flexible liner part vibrations. The Fluistcom geometry induces some additional pressure fluctuations in the cooling passage due to the very flexible section (especially visible around $200-250 \mathrm{~Hz}$ ). The Desire liner due to its high stiffness hardly influences the pressure field inside the cooling passage. Therefore, most likely the noise from the combustion chamber is transmitted to the cooling passage via vibrations of the whole liner walls and not only due to vibrations of the flexible section. 

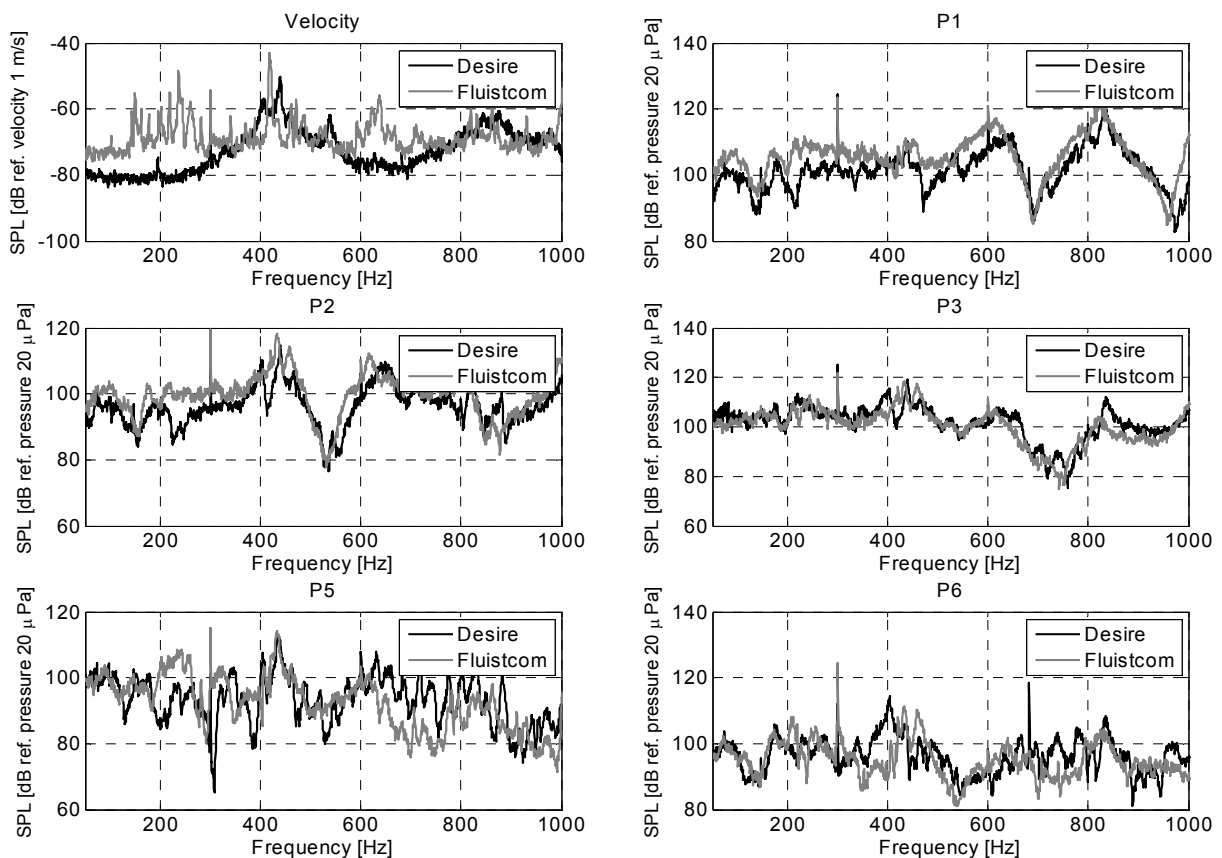

Figure 2-10: Results of experiment with pulsating flow

Next to the results obtained during most of the experiments and discussed above, in Appendix D the data of the experiment which show the thermo-acoustic instabilities near the first acoustic mode is presented. This data confirms calculations of (Van Kampen, 2006) that the instabilities with this combustion chamber geometry may appear near the first or second acoustic eigenfrequency.

\subsection{Flame Transfer FunCtion}

The transfer function describes the linear dependence between a signal excitation in one point and the response of the system in another point. In the combustion process, the transfer function which describes the flame response in term of heat release rate on perturbations upstream of the flame is called the dimensionless flame transfer function (FTF). It is defined according to Equation 2-2. Here $\overline{\dot{Q}}$ and $\overline{\dot{m}_{f}}$ are time mean rates of heat release and fuel mass flow, respectively. $\dot{Q}^{\prime}$ and $\dot{m}_{f}^{\prime}$ are the perturbations per second of the mean values. 
The main sources of the instabilities in a lean premixed turbulence flame are fluctuations in the equivalence ratio, see (Hobson, Fackrell, \& Hewitt, 2000). The mass flow of air exceeds several times the mass flow of fuel during lean combustion, a small perturbation in the fuel flow makes a significant impact on the flame stability, where at the same time the total mass flow of the mixture is hardly perturbed. That is why, for the investigation of the effect of equivalence ratio oscillations on the flame, perturbations of the fuel mass flow rate upstream of the heat source are chosen. The FTF is studied in numerous papers for passive and active flames, see e.g. (Cabot, Vauchelles, Taupin, \& Boukhalfa, 2004) and (Paschereit, Schuermans, Polifke, \& Mattson, 2002), respectively. However, most studies are performed for atmospheric pressure conditions. In this thesis the flame transfer function is investigated at elevated pressure (1.5 bar - 3 bar) for operating points depicted in Table 2-1. Similar operating conditions were investigated in the work of (Van Kampen, 2006) and (Van Kampen \& Kok, 2010) using experimental and numerical data. Here the FTF is obtained entirely with the use of experimental data and thermodynamic relations. Furthermore, the flame transfer function is investigated for two different geometries of the combustion chamber.

Since the instantaneous rate of volume integrated heat release by the flame is not trivial to measure directly, the flame transfer function is factorized in relatively easy to measure relations, see Equation 2-3. These relations multiplied together reconstruct the original FTF.

$$
\begin{gathered}
H_{f}=\frac{\overline{\dot{m}_{f}}}{\dot{\dot{Q}}} \cdot \frac{\dot{Q}^{\prime}}{\dot{m}_{f}^{\prime}} \\
H_{f}=\frac{\overline{\dot{m}_{f}}}{\overline{\dot{Q}}}\left[\frac{\dot{Q}^{\prime}}{M^{\prime}} \cdot \frac{M^{\prime}}{p_{\text {meas }}^{\prime}} \cdot \frac{p_{\text {meas }}^{\prime}}{\delta^{\prime}} \cdot \frac{\delta^{\prime}}{\dot{m}_{f}^{\prime}}\right]
\end{gathered}
$$

For the flame transfer function reconstruction several devices and sensors are employed. The mass flow rate of the fuel is perturbed by the MOOG valve. The MOOG valve obtains information about set amplitude and frequency of pulsation by means of voltage changes ( $\mathrm{V}_{\text {exc }}$ ) from the Siglab device. The voltage signal is translated to displacement $(\delta)$ of the piston located inside the MOOG valve. The movement of the piston results in fluctuations in the fuel mass flow rate, and as a consequence in heat released by the flame. Since heat released by the flame is the main source of sound in the combustion chamber, see (Crighton, Dowling, Ffowcs Williams, Heckl, \& Leppington, 1992) any oscillations in heat released are related directly to the acoustic sound source (M). These oscillations can be 
measured with the use of a chemiluminescence camera and pressure transducers ( $\left.\mathrm{p}_{\text {meas }}\right)$. The layout of the techniques employed to reconstruct the FTF is presented on Figure 2-11.

To retrieve the flame transfer function, steady and pulsating conditions are used. The dynamic measurements are done for the frequency fluctuation in a range from $40 \mathrm{~Hz}$ to 400 $\mathrm{Hz}$ and amplitude of pulsation equal to $7.5 \%$ of the mean equivalence ratio. For the frequency change a sweep-sinusoidal signal generated by the Siglab is used. The resultant transfer function is saved with frequency intervals equal to $5 \mathrm{~Hz}$ (only for the Desire configuration, $7.5 \mathrm{~Hz}$ intervals were used for frequencies below $80 \mathrm{~Hz}$ ). To distinguish the correlated flame response from the flow noise, only results with coherence equal to 0.85 and above are taken into consideration. Both dynamic and steady flow data is averaged over 20 loops. Measurements of $\mathrm{CH}^{*}$ intensity are performed with a sampling frequency equal to $1000 \mathrm{~Hz}$ which limits the $\mathrm{CH}^{*}$ spectrum to $500 \mathrm{~Hz}$. Investigations include different liner configurations and operating points presented in Table 2-1. Since air factor, preheating temperature and mean flow velocity in all cases are the same, the influence on the flame transfer function spectra is limited to the liner geometry and absolute pressure.

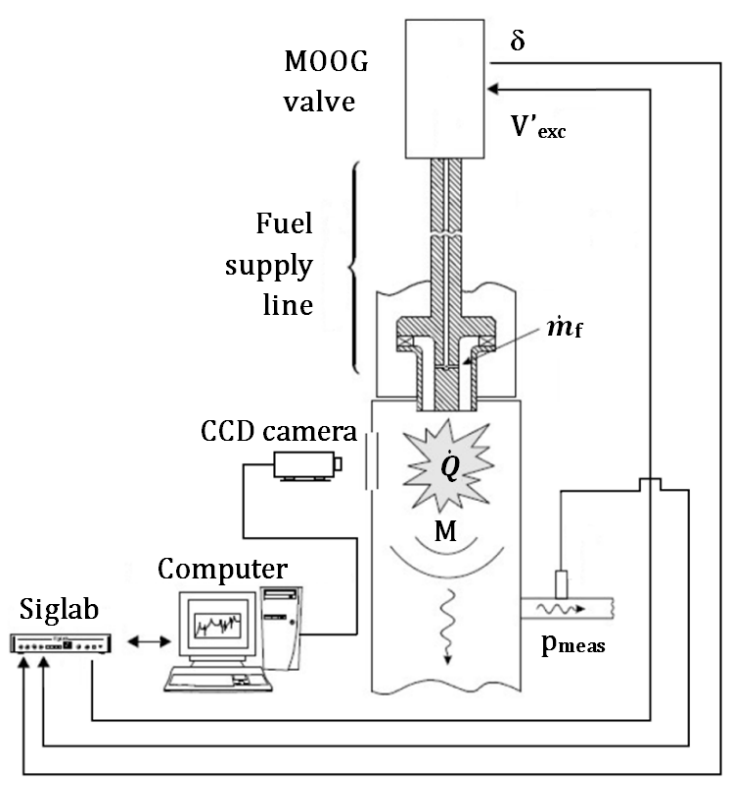

Figure 2-11: Scheme of the main components used to retrieve the FTF 
The first ratio in the bracket in Equation 2-3 is known from thermodynamics as $\frac{\dot{Q}^{\prime}}{M \prime}=$ $\frac{c_{0}^{2}}{\gamma-1} V\left[\frac{m^{5}}{s^{2}}\right]$. It is assumed that the factor $\frac{c_{0}^{2}}{\gamma-1}$ is evaluated at the adiabatic flame temperature and is constant over the flame. This assumption is not entirely correct, however as it has been shown in (Van Kampen, 2006), the induced error is minor.

The second factor i.e. $\frac{M^{\prime}}{p^{\prime} \text { meas }}\left[\frac{s}{m^{2}}\right]$ represents the transfer function between acoustic mass flow and local pressure perturbation per time unit. This function characterises the acoustic behaviour of the combustion system. It can be obtained by performing an experiment at steady flow conditions. Since the concentration of $\mathrm{CH}^{*}$ radicals is linearly proportional to the rate of heat release in the flame, see (Van Kampen, 2006), the spectral shape of the acoustic source is determined from the chemiluminescence measurements of light emitted by excited $\mathrm{CH}^{*}$ radicals. For the spectral shape of pressure oscillations, the auto-spectrum of pressure transducer P1 is used. This auto-spectrum can be contaminated by flow noise. Since the noise, born at a location upstream of the flame e.g. in the burner mouth or at the fuel pipe exit, is observed at both measurement locations, this type of noise is correlated. However, in the auto-spectrum signal also uncorrelated noise created by the flow at the location of the pressure transducer is observed. This flow noise is typically a quadrupole source noise and the flame is a monopole source, see also Chapter 3. Because the contribution of the monopole source in the total sound generation during the combustion process with low Mach number is much bigger than the quadrupole source, as shown in (Klein, 2000), the effect of the flow noise on the obtained results is minor. Furthermore, information about the phase are not included in this transfer function. This because the speed of sound during the combustion process exceeds $900 \mathrm{~m} / \mathrm{s}$ and the distance from the flame to the pressure transducer is about $0.1 \mathrm{~m}$. Hence this phase shift can be neglected for the investigated frequency range.

The transfer function between acoustic flow and local pressure perturbation presented for the Desire and Fluistcom configuration in Figure 2-12 (left) and Figure 2-13 (left), respectively, show similar results. In all cases two peaks in the spectrum are visible around frequencies of $50 \mathrm{~Hz}$ and $150 \mathrm{~Hz}$. They might appear due to acoustic modes of the whole setup, however their magnitude is most likely over-estimated since the flow noise in the low frequency region has higher impact on the results than in the high frequency region.

In the chemiluminescence spectrum see Figure 2-12 (right) and Figure 2-13 (right) the thermo-acoustic instabilities are present in the frequency range $410 \mathrm{~Hz}-460 \mathrm{~Hz}$. This agrees well with the signal obtained from the pressure transducers and presented in Figure 2-8. The frequency and amplitude change of the instabilities are observed even for the same liner geometry. This behaviour is an effect of different temperature distribution inside the combustion chamber during particular measurements. The remaining part of the spectrum 
presents a shape typical to the turbulence noise with a few peaks around the acoustic eigenfrequencies.
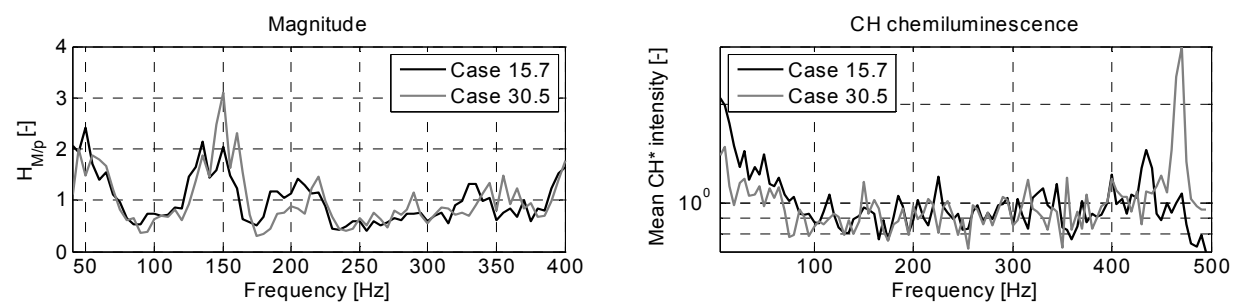

Figure 2-12:Transfer function $\frac{M^{\prime}}{p^{\prime} \text { meas }} / \frac{\bar{M}}{\overline{p_{\text {meas }}}}$ (left) together with $\mathrm{CH}^{*}$ spectrum( right) obtained during investigation of the Desire configuration
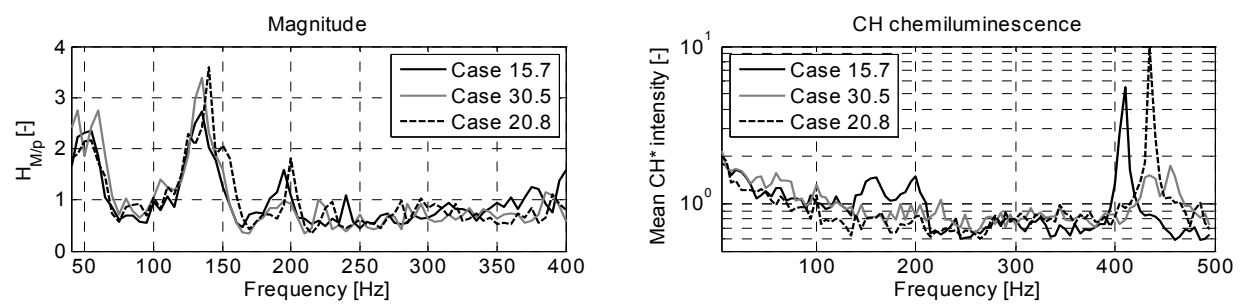

Figure 2-13:Transfer function $\frac{M^{\prime}}{p^{\prime} \text { meas }} / \frac{\bar{M}}{\overline{p_{\text {meas }}}}$ (left) together with $\mathrm{CH}^{*}$ spectrum( right) obtained during investigation of the Fluistcom configuration

The next factor $\frac{p^{\prime} \text { meas }}{\delta \prime}\left[\frac{\mathrm{kg}}{\mathrm{m}^{2} \mathrm{~s}^{2}}\right]$ is obtained from the dynamic combustion experiment. The ratio between the cross-spectrum and auto-spectrum is taken to determine the transfer function between pressure fluctuations at position of pressure transducer $\mathrm{P} 1$ in the combustion chamber and the MOOG valve displacement per time unit. Since the transfer function is calculated as a ratio of the cross-spectrum between two signals and autospectrum of the reference signal, the coherence function can be used to check the quality of the measured data. The signal from the auto-spectrum is assumed to be noise-free due to active perturbation of this channel with the MOOG valve. When the value of the coherence function measured between $\mathrm{P} 1$ and MOOG valve is equal to or higher then 0.85 , than the signal from the transfer function is taken into consideration. In the other case, the results are not evaluated at this particular point. For the Desire data, the coherence was always above the limit. In case of the Fluistcom configuration, $1 \%$ of the results for the operating point 30.5 and $3 \%$ for the operating point 15.7 are not taken into account due to poor coherence. Coherence at operating point 20.8 presents only values above 0.85 . 
The magnitude profile of the $\frac{p^{\prime} \text { meas }}{\delta \prime}$ transfer function is alike for all measurements, see Figure 2-14 and Figure 2-15. Only minor differences in the amplitude of peaks are observed. Introduction of the flexible liner structure and higher absolute pressure introduce minor dissimilarities between the profiles.
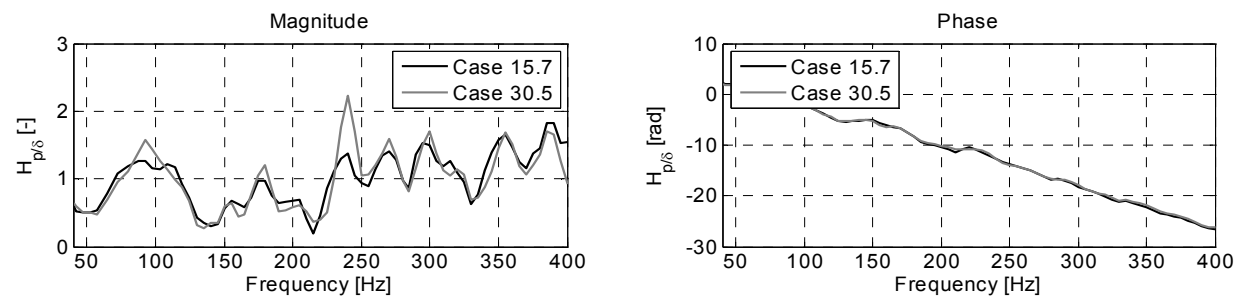

Figure 2-14: Transfer function $\frac{p_{\text {mmeas }}}{\delta \prime} / \frac{\overline{p_{\text {meas }}}}{\bar{\delta}}$ obtained during the Desire investigation
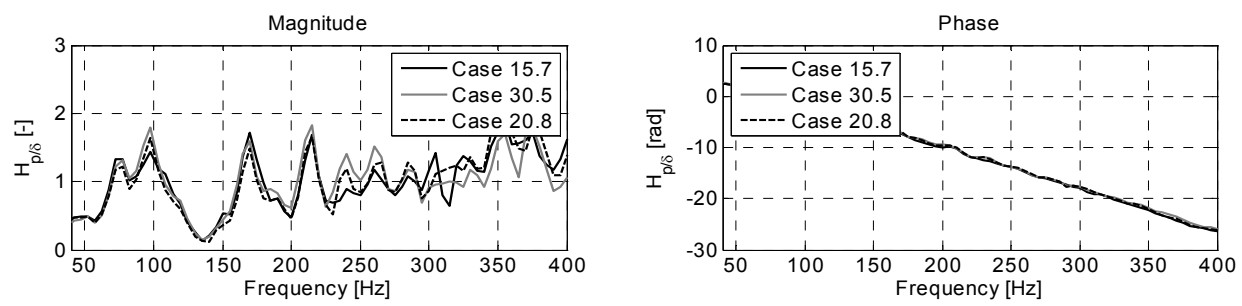

Figure 2-15: Transfer function $\frac{p^{\prime} \text { meas }}{\delta \prime} / \frac{\overline{p_{m e a s}}}{\bar{\delta}}$ obtained during the Fluistcom investigation

The phase spectrum investigated during various conditions presents almost constant time delay behaviour with no clear differences between the investigated operating points and geometries. For all spectra exists a constant time delay between system excitation and response. This can be explained from the fact that all measurements are performed at power to pressure ratio of $83 \mathrm{~kW} / \mathrm{bar}$; hence the flow residence time is constant.

The last transfer function $\frac{\delta \prime}{\dot{m}_{f}}\left[\frac{\mathrm{m} \cdot \mathrm{s}}{\mathrm{kg}}\right]$ represents the transfer of the signal transmitted from the MOOG valve to the mass flow rate of the fuel. This data is received from separate experiment performed and described by (Kleinlugtenbelt, 2005). 

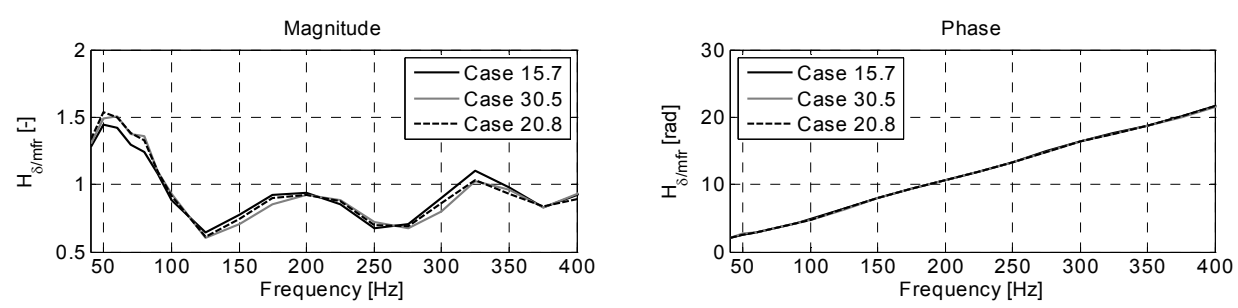

Figure 2-16: Complementary transfer function $\frac{\delta^{\prime}}{\dot{m}_{f}^{\prime}} / \frac{\bar{\delta}}{\dot{\bar{m}}_{f}}$ for both liner configurations

Here again it can be commented that the phase shift depends on the power/pressure ratio (here $83 \mathrm{~kW} / \mathrm{bar}$ ) and the length of the fuel line. Because in Fluistcom the fuel line was longer then in Desire, the results presented in (Kleinlugtenbelt, 2005) have been corrected for this.

The product of all aforementioned transfer functions results in the flame transfer function of the investigated combustion setup presented in Figure 2-17 and Figure 2-18. Here, similar to the earlier investigated transfer functions, only a small difference is observed in the magnitude spectrum. This behaviour is in a good agreement with typical behaviour of transfer functions obtained for combustion systems see e.g. (Rajaram \& Lieuwen, 2002) and (Van Kampen, 2006).

The phase spectrum shows almost linear dependence between phase change and frequency. Again, there is no major difference noticed for all five investigated cases. A constant convective time delay can be calculated for each spectrum by fitting the linear function. For the Desire liner configuration and operating point 15.7 the predicted time delay between mass flow rate fluctuations and flame response is equal to $3.69 \mathrm{~ms}$, whereas for the operating point 30.5 this is $3.60 \mathrm{~ms}$. The same operating points investigated with the use of the Fluistcom liner show time delays equal to $3.77 \mathrm{~ms}$ and $3.71 \mathrm{~ms}$ respectively. In case of the investigation with operating point 20.8 , the time delay is equal to $3.72 \mathrm{~ms}$. From this results it appears that in case of the stiff liner configuration the signal travels faster to the flame front than in case of the Fluistcom geometry. The same behaviour is also observed when experiments are done at higher pressure level. This suggests that for the high absolute pressure and stiff liner configuration the flame is located closer to the burner mouth than during the remaining investigated cases. Nevertheless these differences, it can be concluded that at constant mean velocity, air factor and preheated air temperature, the effect of pressurizing the combustion chamber and increasing liner flexibility is not significant for the FTF. In all investigated cases, the magnitude and spectrum of the flame transfer function is almost identical. 

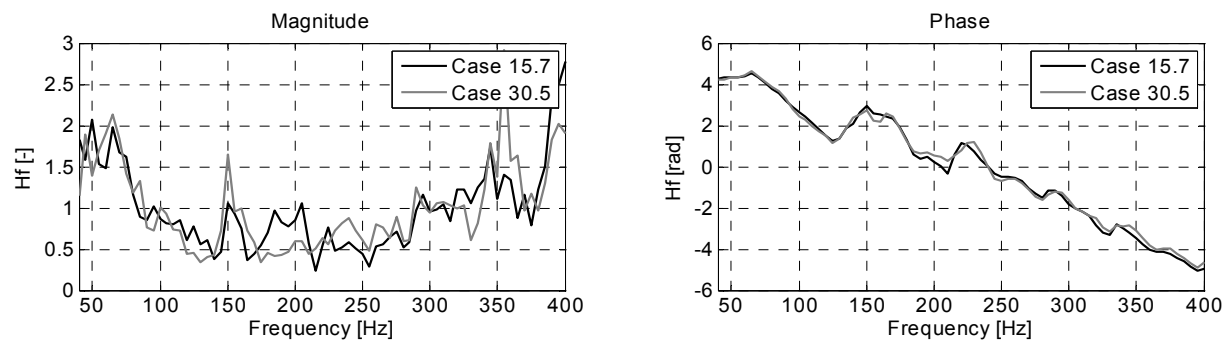

Figure 2-17: Flame transfer function of the Desire configuration
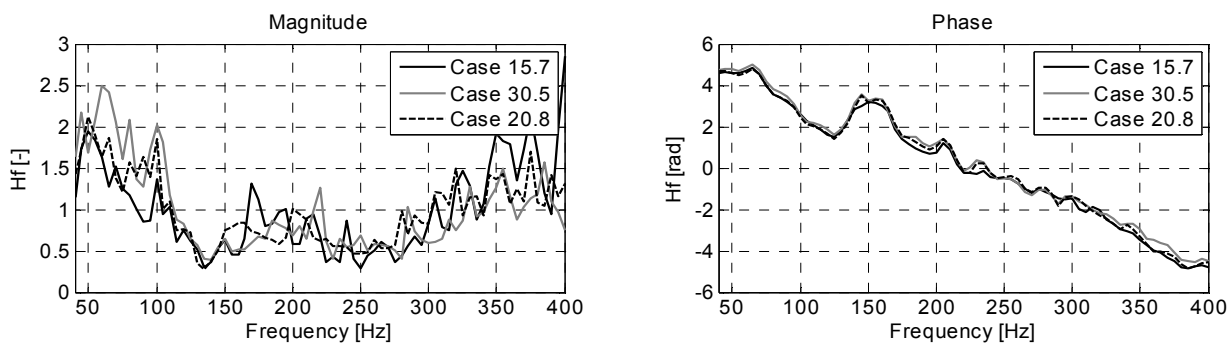

Figure 2-18: Flame transfer function of the Fluistcom configuration

\subsection{Conclusions}

The experimental results of the combustion process under steady and pulsating flow conditions are presented here. Two liner configurations and several operating points are investigated. The influence of the liner geometry on the vibration level and the acoustic waves inside the combustion chamber and cooling passage is explored. Furthermore, the flame transfer function at various elevated absolute pressures is retrieved. Since in the current setup configuration it is not possible to measure the flame transfer function directly, the relation between flow perturbation and heat released by flame is reconstructed from the transfer functions measured at various locations in the test rig.

The results of the pressure fluctuations inside the combustion chamber and cooling passage are similar for both liner configurations. The thermo-acoustic instabilities in case of the flexible liner configuration (Fluistcom) are shifted slightly to lower frequencies in comparison to the stiff liner geometry (Desire). Since during the experiment some changes in the instantaneous temperature profile inside the combustion chamber and cooling passage are observed, the thermo-acoustic instabilities even during investigation of the 
same operating conditions and liner configuration vary slightly. This behaviour is observed for both, steady and pulsating flow measurement. Nevertheless these inaccuracies, the instabilities visible during the investigation of the Fluistcom geometry are located within a frequency range of $405 \mathrm{~Hz}-431 \mathrm{~Hz}$, whereas for the Desire liner they are placed between $433 \mathrm{~Hz}$ and $458 \mathrm{~Hz}$.

The liner vibrations present a similar behaviour as the pressure fluctuations, i.e. the main vibrations of the Fluistcom liner are located at lower frequencies in comparison to the Desire liner. Furthermore, due to lower stiffness of the Fluistcom liner, the vibrations are also well visible at acoustic eigenfrequencies. This is not always the case for the Desire geometry.

In the cooling chamber, the pressure spectrum signal is much undulated. However, it still resembles the acoustic profile visible in the combustion chamber. Therefore, the sound is probably transmitted from the combustion chamber to the cooling passage not only by the liner vibrations (flexible section and the whole liner) also by the bypass connection for the exhaust gas cooling. The undulation of the signal is induced by obstacles present in the cooling passage (e.g. thermocouples, connections for the side tubes etc.). These obstacles make the flow more turbulent and as a result the acoustics inside the cooling passage is enhanced.

The flame transfer function is reconstructed from the individual transfer function factorizations between various points in the combustion system. The experiments show minor influence of the liner configuration and pressure level on the obtained results. All obtained spectra have similar profiles in magnitude and phase. This is explained by the fact that all operating points have a constant power to pressure ratio. Furthermore, there is almost a linear correlation between phase change and frequency. These results interpreted for the time delay show that the Desire liner configuration and higher absolute pressure reduce slightly time delay necessary to transfer disturbances from the source to the front of the flame. 


\section{CFD OF The Combusting Flow}

\subsection{INTRODUCTION}

This chapter is devoted to combustion process modelling in the CFD analysis. The RANS approach is used to resolve the turbulent, combustible flow field. Two different combustion models are investigated, which are standard available in the CFX commercial code, the Eddy Dissipation and Finite Rate Chemistry model and the UT in-house combustion model CFI. The effect of turbulence is calculated using k- $\varepsilon$ and SAS turbulence models. Steady-state and transient calculations are performed to obtain information about flame properties and acoustic wave propagation. The results of the transient calculations are used as input conditions for further fluid-structure interaction and acousto-elastic analyses. Pulsation of the equivalence ratio during the transient flow is performed in order to match with the experimental conditions. The CFX commercial code from Ansys, versions 5.7, 10 and 11 are used for the CFD computations. Numerical results are compared to experimental data for one of the operating points for validation.

\subsection{BACKGROUND}

The results presented in this chapter are obtained using Computational Fluid Dynamics (CFD). With CFD complicated fluid flow problems are resolved by numerical methods. In CFD, flow features are described by a set of partial differential equations (PDE). Distribution of the flow properties is obtained by domain discretization and numerical solution of the PDEs. Therefore to get an accurate solution the mathematical model of the flow domain with boundary conditions must be properly formulated. This requires specification of the governing equations, boundary conditions and coordinate system. If all conditions are satisfied, the mathematical problem which has to be solved is considered as well posed, and can be solved numerically. 
Most of the numerical codes are based on simplifications of the Navier-Stokes equations which are being solved using the Finite Volume Method, Finite Element Method (FEM) or Finite Difference Method. The latter is not investigated in this thesis. Information about the Finite Difference Method can be found in (Mitchell, 1980). In this thesis, the FEM is limited to computations of the liner vibrations and to the acousto-elastic analysis. The application of the FEM in the CFD is described by (Baker, 1983), (Zienkiewicz, Taylor, \& Zhu, 2005).

One of the well-known and common methods to resolve the PDEs in the CFD analysis is the Finite Volume Method, see e.g. (Versteeg \& Malalasekera, 2007), (Wesseling, 2001). In this method instead of discretization of the PDEs, the integral, fundamental form of the equations is solved. Moreover, the governing equations are not approximated at the grid points, but approximation is done by means of balances over the element volume. The governing equations describing a time-dependent viscous combustible flow problem are the following:

Continuity:

$$
\frac{\partial \rho}{\partial t}+\frac{\partial \rho u_{i}}{\partial x_{i}}=0
$$

Momentum:

$$
\frac{\partial \rho u_{j}}{\partial t}+\frac{\partial}{\partial x_{i}}\left(\rho u_{i} u_{j}\right)=-\frac{\partial p}{\partial x_{j}}+\frac{\partial \tau_{i j}}{\partial x_{i}}
$$

Species:

$$
\frac{\partial \rho Y_{k}}{\partial t}+\frac{\partial}{\partial x_{i}}\left(\rho u_{i} Y_{k}\right)=-\frac{\partial}{\partial x_{i}}\left(V_{k, i} Y_{k}\right)+\dot{\omega}_{k}
$$

Enthalpy:

$$
\frac{\partial \rho h_{s}}{\partial t}+\frac{\partial}{\partial x_{i}}\left(\rho u_{i} h_{s}\right)=\frac{D p}{D t}-\frac{\partial q_{i}}{\partial x_{i}}+\tau_{i j} \frac{\partial u_{i}}{\partial x_{j}}+\dot{\omega}_{T}
$$

The conservation of energy equation here is written in the form of enthalpy conservation, see Equation 3-4. The flow viscous tensor and the energy heat flux are indicated with $\tau_{i j}$ and $q_{i}$ respectively. They can be modelled with the classic Newtonian fluid constitutive equation and the Fourier heat transfer law, as shown in Equation 3-5 for $\tau_{i j}$ and Equation 3- 6 for $q_{i}$.

$$
\tau_{i j}=-\frac{2}{3} \mu \frac{\partial u_{k}}{\partial x_{k}} \delta_{i j}+\mu\left(\frac{\partial u_{i}}{\partial x_{j}}+\frac{\partial u_{j}}{\partial x_{i}}\right)=-\left(P_{i j}-p \delta_{i j}\right)
$$




$$
q_{i}=-\lambda \frac{\partial T}{\partial x_{i}}+\rho \sum_{k=1}^{N} V_{k, i} Y_{k} h_{s, k}
$$

Where: $\delta_{i j}$ is the Kronecker symbol equal to 1 when $i=j$, otherwise $\delta_{i j}=0 . Y_{K}$ is the mass fraction, $V_{k, i}$ are the diffusion velocities, $\dot{\omega}_{k}$ is reaction rate of species $k, \dot{\omega}_{T}$ is heat release due to combustion described as: $\dot{\omega}_{T}=-\sum_{k=1}^{N} \Delta h_{f, k}^{0} \dot{\omega}_{k}$. Enthalpy $h_{s}$ is the sensible enthalpy written in the form given by (Poinsot \& Veynante, 2005).

\subsection{Turbulent Combustion}

Providing a numerical solution up to the smallest turbulent structures, the NavierStokes equations are capable to predict the turbulent flow in all its complexity. Unfortunately up to now, such accuracy is extremely time-consuming even for a relatively simple case. The barrier is an insufficient computational power to resolve non-linear, second-order, multidimensional and highly interrelated equations. Methods like DNS (Direct Numerical Simulations) which solve the exact Navier-Stokes equations are limited mostly to very simple cases without combustion at relatively modest Reynolds numbers. Thus, in order to predict the high turbulent reacting flow behaviour in complex geometries, models based on the original Navier-Stokes equations were developed. Instead of representing the turbulence exactly in time and spatial scale, models for the statistical approximation of the effect of the turbulence on the mean or averaged flow properties are used. The introduction of the turbulence models reduces the accuracy of the solution to the accurateness of the turbulence model, but at the same time decreases significantly computational effort and overall calculation costs. The two most popular models used for numerical computations are Large Eddy Simulation (LES) and Reynolds Averaged NavierStokes (RANS). Both require less computational effort than DNS, but they introduce approximations in the resolved equations. The effect of different turbulence models on the turbulence field is presented in Figure 3-1. 


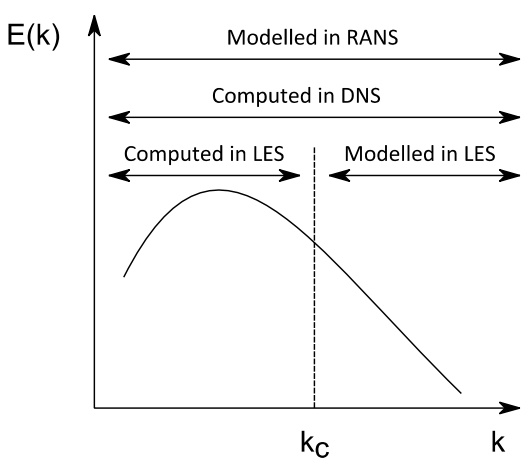

Figure 3-1: DNS, LES and RANS in CFD. Here: $E(k)$ is turbulence energy spectrum, $k$ is wave number and $k_{C}$ is cut-off wave number (taken from (Poinsot \& Veynante, 2005))

\subsubsection{LES}

In the LES, the problem which is investigated is described by filtering the transient governing equations. Filtering is basically a manipulation of the exact Navier-Stokes equations to limit the spatial resolution to phenomena (e.g. eddies) larger than the size of the filter, which is usually taken as the mesh size, as it is shown in (Poinsot \& Veynante, 2005). The filtered operation done on the flow quantities: $Q(\boldsymbol{x}, t)$ is defined by Equation 3-7:

$$
\bar{Q}(x)=\int Q\left(x^{*}\right) F\left(x-x^{*}\right) d x^{*}
$$

Where: $F$ is the LES filter.

The filtering operation allows the LES to resolve turbulence structures larger than the mesh size directly, whereas small turbulence structures are modelled by the use of subgrid closure rules. The assumption is made that the large eddies of the turbulent flow have a major anisotropic effect on the flow due to their three-dimensionalities and timedependence. Therefore they have to be calculated directly. Whereas, the small eddies, which derive their energy from the large ones, are of such a small scale (isentropic) and disappear so quickly that is impractical to resolve them directly a reasonably small time scale and grid size. It means that it is not necessary to decrease the mesh size further to capture small eddies, but the small structures can be modelled by the subgrid-scale stress model. 
The main advantage of the LES formulation in comparison to the RANS approach is a reduced impact of the modelling in the resolved flow. However, necessity of modelling a $3 \mathrm{D}$ domain with high grid resolution to capture all geometry-dependent eddies in time dependent flow make this calculation still very expensive in the meaning of CPU time and storage space.

The LES approach is not performed during the current investigation and therefore it is not described here in details. Detailed information about the LES scheme can be found in (Poinsot \& Veynante, 2005).

\subsubsection{RANS}

The computational effort to perform the RANS computation is much smaller than LES. Simplifications of the flow and the flow domain by introduction of 2D flows, symmetry or periodic boundary conditions, increasing the numerical grid size, reduces even more the cost of calculations. The significant drawback of the RANS approach is that all turbulence phenomena have to be model instead of resolving them directly. The high robustness and short calculation time combined with reasonable accuracy compensate this drawback and make the RANS method one of most used numerical tools for industrial and scientific computations. Taking into consideration that simultaneously calculations of fluid and structural domain can be combined with data exchange between solvers, through interface connection, it makes sense why the work presented in this thesis is limited to the RANS approach. The same calculations using the LES method would enormously increase the total calculation time.

The idea behind the RANS model is to split the turbulent quantities $Q(x, t)$ in their ensemble averaged values $(\bar{Q})$ and the turbulent fluctuating components $\left(Q^{\prime}\right)$ according to Equation 3-8. This is called Reynolds decomposition.

$$
\begin{gathered}
Q=\bar{Q}+Q^{\prime} \\
\bar{Q}(t, x)=\frac{1}{T} \int_{-\frac{T}{2}}^{\frac{T}{2}} Q(t+\tau, x) d \tau
\end{gathered}
$$

In Equation 3-9 the quantity $T$ is a period that is large compared with the time scale of turbulence, but small in relation to other time dependent features of the flow, see 
(Wesseling, 2001). In fully developed turbulent flows the time scale of the turbulent fluctuations is often very small compared to the time scale of other unsteady processes. In order to describe accurately flows with large density changes, like in combustion, the density-weighted mean or so-called Favre average quantity $(\tilde{Q})$ together with corresponding fluctuations $\left(Q^{\prime \prime}\right)$ are introduced, see Equation 3-10 to Equation 3-12.

$$
\begin{gathered}
\tilde{Q}=\frac{\overline{\rho Q}}{\bar{\rho}} \\
\bar{Q}(t, x)=\frac{1}{\bar{\rho} T} \int_{-\frac{T}{2}}^{\frac{T}{2}} \rho Q^{\prime}(t+\tau, x) d \tau \\
Q^{\prime \prime}=Q-\tilde{Q}
\end{gathered}
$$

The definition of period $T$ in Equation 3-9 allows the occurrence of low frequency pulsations and oscillations in the resolved flow. Those fluctuations have no influence on the turbulence structures, as the latter occur with much higher characteristic frequency. Therefore the large-scale organised unsteady motions are resolved by the numerical scheme, and the small structures of the turbulences are captured by RANS modelling. The unsteady or transient RANS is called URANS or TRANS.

The ensemble averaged Navier-Stokes equations can be rewritten in terms of the averaged quantities. For the adiabatic, compressible and unsteady flow of reactive gases, the RANS equations for conservation of mass, momentum, species and enthalpy can be rewritten (in this thesis always in Cartesian coordinates) in the form:

$$
\begin{gathered}
\frac{\partial \bar{\rho}}{\partial t}+\frac{\partial \bar{\rho} \widetilde{u}_{l}}{\partial x_{i}}=0 \\
\frac{\partial \bar{\rho} \tilde{u}_{j}}{\partial t}+\frac{\partial}{\partial x_{i}}\left(\bar{\rho} \tilde{u}_{i} \widetilde{u}_{\jmath}\right)=-\frac{\partial \bar{p}}{\partial x_{j}}+\frac{\partial}{\partial x_{i}}\left(\bar{\tau}_{i j}-\overline{\rho u_{\imath}^{\prime \prime} u_{\jmath}^{\prime \prime}}\right) \\
\frac{\partial \bar{\rho} \tilde{Y}_{k}}{\partial t}+\frac{\partial}{\partial x_{i}}\left(\bar{\rho} \tilde{u}_{i} \tilde{Y}_{k}\right)=-\frac{\partial}{\partial x_{i}}\left(\overline{V_{k, l} Y_{k}}+\bar{\rho} \widetilde{u_{l}^{\prime \prime} Y_{k}^{\prime \prime}}\right)+\overline{\omega_{k}}
\end{gathered}
$$




$$
\begin{aligned}
\frac{\partial \bar{\rho} \tilde{h}_{s}}{\partial t}+\frac{\partial}{\partial x_{i}}\left(\bar{\rho} \tilde{u}_{i} \tilde{h}_{s}\right) & \\
= & \frac{\overline{D p}}{D t}+\frac{\partial}{\partial x_{i}}\left(\overline{\lambda \frac{\partial T}{\partial x_{\imath}}}-\overline{\rho u_{\imath}^{\prime \prime} h_{s}^{\prime \prime}}-\rho \sum_{k=1}^{N} \overline{V_{k, l} Y_{k} h_{s, k}}\right) \\
& +\overline{\tau_{l \jmath} \frac{\partial u_{l}}{\partial x_{\jmath}}}+\overline{\dot{\omega}_{T}}
\end{aligned}
$$

The unclosed terms contain products of fluctuating values i.e. $\overline{\rho u_{\imath}^{\prime \prime} u_{\jmath}^{\prime \prime}}$ and $\bar{\rho} \widetilde{u_{l}^{\prime \prime} Y_{k}^{\prime \prime}}$, that need to be modelled. These terms are the Reynolds stress term and the turbulent species flux. The closure relations make generally use of extra transport equations which involve averaged flow quantities. One of the most common ways to model the Reynolds stresses is the assumption that turbulent stresses are proportional to the mean velocity gradient via the concept of a turbulent eddy viscosity (eddy viscosity modelling). According to the Boussinesq approximation, defined by Equation 3-17, the problem is then moved to the definition of the turbulent eddy viscosity. Species fluxes are closed with the use of the classical gradient assumption defined in Equation 3-18 linking the diffusion coefficient to the eddy viscosity by means of a turbulent Schmidt number.

$$
\begin{gathered}
-\overline{\rho u_{\imath}^{\prime \prime} u_{\jmath}^{\prime \prime}}=\mu_{t}\left(\frac{\partial \widetilde{u_{l}}}{\partial x_{j}}+\frac{\partial \widetilde{u_{j}}}{\partial x_{i}}\right)-\frac{2}{3}\left(\tilde{\rho} k+\mu_{t} \frac{\partial \widetilde{u_{k}}}{\partial x_{k}}\right) \delta_{i j} \\
-\bar{\rho} \widetilde{u_{\imath}^{\prime \prime} Y_{k}^{\prime \prime}}=\frac{\mu_{t}}{S c_{k t}} \frac{\partial \tilde{Y}_{k}}{\partial x_{i}}
\end{gathered}
$$

The eddy viscosity has the dimension of a square length per time unit and is hence univocally determined by a typical turbulent velocity and length scale. The velocity scale is often modelled using an extra transport equation for the turbulent kinetic energy defined as: $k=\frac{1}{2} \widetilde{u_{\imath}^{\prime \prime} u_{\imath}^{\prime \prime}}$. The length scale is calculated as a function of the turbulence kinetic energy dissipation $(\varepsilon)$ or the turbulent kinetic frequency $(\omega)$. Two-equation family models: $k-\varepsilon$ and $\mathrm{k}-\omega$ are considered depending on the viscosity formulation. The results of calculations with various turbulent models are compared with the experimental and literature data in this thesis. Therefore they are shortly described below. Numerical constants of the model are presented in Chapter 6, where results of calculations for the simple geometry with the use of the k- $\varepsilon, k-\omega$ and SST model are compared. For the CFD part of the fluid-structure interaction, mainly the $\mathrm{k}-\varepsilon$ and SAS models are applied. 
- The k- $\varepsilon$ model described by (Launder \& Spalding, 1974) assumes that the eddy viscosity is related to the turbulence kinetic energy and turbulence dissipation rate according to Equation 3-19. Values of the turbulent kinetic energy and turbulence dissipation rate are obtained directly from the differential transport equations of both variables.

$$
\begin{gathered}
\mu_{t}=C_{\mu} \bar{\rho} \frac{k^{2}}{\varepsilon} \\
\frac{\partial}{\partial t}(\bar{\rho} k)+\frac{\partial}{\partial x_{i}}\left(\bar{\rho} \widetilde{u}_{\imath} k\right)=\frac{\partial}{\partial x_{i}}\left[\left(\mu+\frac{\mu_{t}}{\sigma_{k}}\right) \frac{\partial k}{\partial x_{i}}\right]+P_{k}-\bar{\rho} \varepsilon \\
\frac{\partial}{\partial t}(\bar{\rho} \varepsilon)+\frac{\partial}{\partial x_{i}}\left(\bar{\rho} \tilde{u}_{i} \varepsilon\right)=\frac{\partial}{\partial x_{i}}\left[\left(\mu+\frac{\mu_{t}}{\sigma_{\varepsilon}}\right) \frac{\partial \varepsilon}{\partial x_{i}}\right]+C_{\varepsilon 1} \frac{\varepsilon}{k} P_{k}-C_{\varepsilon 2} \bar{\rho} \frac{\varepsilon^{2}}{k}
\end{gathered}
$$

The source term $P_{k}$ is given by: $P_{k}=-\bar{\rho} \overline{u_{\imath}^{\prime \prime} u_{\jmath}^{\prime \prime}} \frac{\partial \widetilde{u_{l}}}{\partial x_{j}}$

The robustness and high accuracy, as well as low computational costs make the model an important tool, especially in industrial applications. The drawback of the model is a poor prediction of the flow behaviour in case of non-equilibrium boundary layers. The reattachment point during separation flow calculations is usually under-predicted, as shown in (Pozarlik, Panara, Kok, \& van der Meer, 2008). Errors occur in the magnitude of the local heat transfer and as a consequence the overall performance is not predicted correctly.

- The k- $\omega$ model see for example (Wilcox, 1986) was developed in order to improve the predictions in the near wall region and reduce the errors in adverse pressure gradient calculations. In order to define the turbulent eddy viscosity, the k- $\omega$ model uses a frequency scale $(\omega)$ called also specific turbulent dissipation rate. The eddy viscosity in this model is defined as Equation 3-22. Both turbulent kinetic energy and specific dissipation rate are obtained from the solution of the following transport equations formulated by Wilcox:

$$
\mu_{t}=\bar{\rho} \frac{k}{\omega}
$$




$$
\begin{gathered}
\frac{\partial}{\partial t}(\bar{\rho} k)+\frac{\partial}{\partial x_{i}}\left(\bar{\rho} \tilde{u}_{\imath} k\right)=\frac{\partial}{\partial x_{i}}\left[\left(\mu+\frac{\mu_{t}}{\sigma_{k}}\right) \frac{\partial k}{\partial x_{i}}\right]+P_{k}-\beta^{\prime} \bar{\rho} k \omega \\
\frac{\partial}{\partial t}(\bar{\rho} \omega)+\frac{\partial}{\partial x_{i}}\left(\bar{\rho} \tilde{u_{\imath}} \omega\right)=\frac{\partial}{\partial x_{i}}\left[\left(\mu+\frac{\mu_{t}}{\sigma_{\omega}}\right) \frac{\partial \omega}{\partial x_{i}}\right]+\alpha \frac{\omega}{k} P_{k}-\beta \bar{\rho} \omega^{2}
\end{gathered}
$$

The major advantage of the $\mathrm{k}-\omega$ model is the robust and simple way how the near wall region is handled. Contrary to the $\mathrm{k}-\varepsilon$ model, the $\mathrm{k}-\omega$ model does not involve complex non-linear damping functions to take into account the near wall lowReynolds effects. Due to the non-linearities of the low-Re damping functions, the $\mathrm{k}-\varepsilon$ models typically require a higher near wall resolution than the $\mathrm{k}-\omega$ model (i.e. for the $k-\varepsilon$ low-Re models, a near wall resolution of $y+<0.2$ is often required whereas for the $\mathrm{k}-\omega$ model a less strict condition of $\mathrm{y}+<2$ is sufficient). In complicated industrial applications it is often impossible to resolve with such a grid resolution the boundary layer. Therefore the wall function approach is still very popular. In order to improve the accuracy of industrial calculations the k- $\omega$ model implemented in the CFX code has the ability to smoothly shift between low-Re number formulation and a wall function scheme depending on the wall $\mathrm{y}+$ resolution. The main weakness of the $\mathrm{k}-\omega$ model is the strong sensitivity of the solution to the free stream $\omega$ values.

- In order to gather the best from the k- $\varepsilon$ and the k- $\omega$ models, a new blended model called SST (Shear Stress Transport) model was developed. The SST model calculates the flow in the near wall region using a $k-\omega$ formulation whereas in the bulk flow the high Reynolds k- $\varepsilon$ formulation is employed. A smooth transition between the two formulations is ensured by the use of additional blending factors which are functions of the wall distance. To avoid excessive shear stress values in adverse pressure gradient conditions, the turbulent shear stress in the boundary layer is limited based on the Bradshaw assumption of direct proportionality with the kinetic energy $\tau=\rho a_{1} k$. In the SST model eddy viscosity is resolved according to Equation 3-25. As in the k- $\omega$ model, the CFX implementation of the SST model permits the automatic shift from the low-Re number formulation to the wall function scheme according to the grid resolution.

$$
\mu_{t}=\frac{a_{1} k}{\max \left(a_{1} \omega ; S F_{2}\right)}
$$




\subsubsection{SAS}

Recently, a new turbulence model/approach was implemented into the CFX commercial code. This approach is the Scale Adaptive Simulation also known as SAS. The model was introduced firs by Menter et al. (Menter, Kuntz, \& Bender, 2003). The SAS simulation is a URANS approach but with behaviour like LES. Therefore a new section is here dedicated to this model. The main advantage of this model is a high ability to capture unsteady flow features, more precisely than standard URANS, see for example: (Menter \& Egorov, 2005), (Withag, 2007), (Pozarlik \& Kok, 2008). At the same time, the model provides standard RANS capabilities in stable flow regions (CFX Ansys 11, 2007). In the SAS model the van Karman length-scale, in the form presented in Equation 3-26, was introduced into the exact transport equation for the integral length-scale as derived by (Rotta, 1972). The information provided by the von Karman length-scale allows the model to dynamically adjust in the URANS simulation. The transport equations of the SAS model are Equation 3-27 and Equation 3-28.

$$
\begin{gathered}
L_{v K}=\kappa\left|\frac{U^{\prime}}{U^{\prime \prime}}\right| \\
\frac{\partial \rho k}{\partial t}+U_{j} \frac{\partial \rho k}{\partial x_{j}}=P_{k}-c_{\mu}^{3 / 4} \rho \frac{k^{2}}{\Phi}+\frac{\partial}{\partial y}\left[\frac{\mu_{t}}{\sigma_{k}} \frac{\partial k}{\partial y}\right] \\
\frac{\partial \rho \Phi}{\partial t}+\frac{\partial \rho U_{j} \Phi}{\partial x_{j}}=\xi_{1} \frac{\Phi}{k} P_{k}-\xi_{2} \mu_{t} S\left|U^{\prime \prime}\right| \frac{\Phi^{2}}{k^{3 / 2}}-\xi_{2} \rho k+\frac{\partial}{\partial y}\left[\frac{\mu_{t}}{\sigma_{\Phi}} \frac{\partial \Phi}{\partial y}\right]
\end{gathered}
$$

Where: $\left|U^{\prime}\right|=S$ and $\left|U^{\prime \prime}\right|=\sqrt{\frac{\partial^{2} U_{i}}{\partial x_{k}^{2}} \frac{\partial^{2} U_{i}}{\partial x_{j}^{2}}}, S$ is the absolute value of the strain rate. Moreover $\Phi=\sqrt{k} L$ and $v_{t}=c_{\mu}^{1 / 4} \Phi$. Constant used in the SAS model are as follows:

\begin{tabular}{ccccccc}
$\boldsymbol{\xi}_{\mathbf{1}}$ & $\boldsymbol{\xi}_{\mathbf{2}}$ & $\boldsymbol{\xi}_{\mathbf{3}}$ & $\boldsymbol{\sigma}_{\boldsymbol{k}}$ & $\boldsymbol{\sigma}_{\boldsymbol{\Phi}}$ & $\boldsymbol{\kappa}$ & $\boldsymbol{c}_{\boldsymbol{\mu}}$ \\
\hline 0.8 & 0.0326 & 3.51 & $2 / 3$ & $2 / 3$ & 0.41 & 0.09
\end{tabular}

Table 3-1: Model constants 
Contrary to standard URANS models, the SAS formulation provides a turbulent lengthscale, which is not proportional to the thickness of the turbulent layer, but it is proportional to the local flow structure. Due to the fact, that the SAS-relevant term is $\left|U^{\prime \prime}\right|$, the length scale $L$ predicted by the model is largely proportional to the von Karman length scale. The reason is that $L_{v K}$ adjusts to the already resolved scales in a simulation and provides a length-scale, which is proportional to the size of the resolved eddies. Standard turbulence models, on the other hand always provide a length-scale proportional to the thickness of the shear layer. They do not adjust to the local flow topology and are therefore overly diffusive, as shown by (Menter, Kuntz, \& Bender, 2003).

In order to provide SAS capability to the SST model, Equation 3-28 is transformed to the $\mathrm{k}-\omega$ framework using the relation: $\Phi=\frac{1}{c_{\mu}^{1 / 4}} \frac{k}{\omega}$. The resulting equation is as follows:

$$
\begin{aligned}
\frac{\partial \rho \Phi}{\partial t}+\frac{\partial \rho U_{j} \omega}{\partial x_{j}}= & \alpha \rho S^{2}-\beta \rho \omega^{2}+\frac{\partial}{\partial x_{j}}\left(\frac{\mu_{t}}{\sigma_{\omega}} \frac{\partial \omega}{\partial x_{j}}\right) \\
& +\frac{2 \rho}{\sigma_{\Phi}}\left(\frac{1}{\omega} \frac{\partial k}{\partial x_{j}} \frac{\partial \omega}{\partial x_{j}}-\frac{k}{\omega^{2}} \frac{\partial \omega}{\partial x_{j}} \frac{\partial \omega}{\partial x_{j}}\right)+\widetilde{\xi}_{2} \kappa \rho S^{2} \frac{L}{L_{v K}} \\
& +\left[\frac{\rho \omega}{k} \frac{\partial}{\partial x_{j}}\left(\frac{v_{t}}{\sigma_{\omega}} \frac{\partial k}{\partial x_{j}}\right)\left(\frac{1}{\sigma_{k}}-\frac{1}{\sigma_{\Phi}}\right)\right]
\end{aligned}
$$

The first three terms on the RHS are standard terms of the original k- $\omega$ model, see (Wilcox, 1986). Term: $\frac{2 \rho}{\sigma_{\Phi}}\left(\frac{1}{\omega} \frac{\partial k}{\partial x_{j}} \frac{\partial \omega}{\partial x_{j}}\right)$ is the cross-diffusion term and it is already included in the SST model to help the model prevent the free stream sensitivity. The last term: $\left(\frac{1}{\sigma_{k}}-\frac{1}{\sigma_{\Phi}}\right)$ is equal to zero, as both the k- $\omega$ and the $\mathrm{k}-\Phi$ model use identical diffusion constants in both equations. The remaining terms are therefore defined as:

$$
F_{S A S-S S T}=-\frac{2 \rho}{\sigma_{\Phi}}\left(\frac{k}{\omega^{2}} \frac{\partial \omega}{\partial x_{j}} \frac{\partial \omega}{\partial x_{j}}\right)+\widetilde{\xi}_{2} \kappa \rho S^{2} \frac{L}{L_{v K}}
$$

It is the goal of the transformation to preserve the SST model in the RANS regime and to activate the SAS capability in URANS regions. In the RANS regime both terms on the RHS of Equation 3-30 are of the same size, whereas the $L_{v K}$ term dominates in the SAS regime: 
RANS regime

$\frac{2 \rho}{\sigma_{\Phi}}\left(\frac{k}{\omega^{2}} \frac{\partial \omega}{\partial x_{j}} \frac{\partial \omega}{\partial x_{j}}\right) \approx \widetilde{\xi}_{2} \kappa \rho S^{2} \frac{L}{L_{v K}}$

SAS regime

$$
\frac{2 \rho}{\sigma_{\Phi}}\left(\frac{k}{\omega^{2}} \frac{\partial \omega}{\partial x_{j}} \frac{\partial \omega}{\partial x_{j}}\right)<\widetilde{\xi}_{2} \kappa \rho S^{2} \frac{L}{L_{v K}}
$$

In order to preserve the SST model in the RANS region, the $\mathrm{F}_{\mathrm{SAS}-\mathrm{SST}}$ term is modelled according to Equation 3-33.

$$
\begin{aligned}
& F_{S A S-S S T}=\rho F_{S A S} \max {\left[\widetilde{\xi_{2}} \kappa \rho S^{2} \frac{L}{L_{v K}}-\frac{2 k}{\sigma_{\Phi}}\right.} \\
&\left.\cdot \max \left(\frac{1}{\omega^{2}} \frac{\partial \omega}{\partial x_{j}} \frac{\partial \omega}{\partial x_{j}}, \frac{1}{k^{2}} \frac{\partial k}{\partial x_{j}} \frac{\partial k}{\partial x_{j}}, 0\right)\right]
\end{aligned}
$$

Where: $\widetilde{\xi}_{2}=\xi_{2} c_{S A S}, c_{S A S}=1.755, F_{S A S}=1.25$. The constant $F_{S A S}$ has been introduced for the calibration of the SAS term in the SST environment.

Because the SAS model is combined always with the SST model, for convenience, it is called SAS in the further part of the thesis.

\subsection{Combustion Models}

Two different combustion models were used for combustion process modelling: Eddy Dissipation/Finite Rate Chemistry model, available in the CFX code and the CFI combustion model developed in the Group of Thermal Engineering.

For the reacting flow calculations, the standard CFX single-step model of a reaction can be used. The model determines the rate at which a component is consumed or created in a single reaction step during the combustion process. The Eddy Dissipation/Finite Rate Chemistry (ED/FRC) model can be used to calculate reactions occurring in the flame. This model is a combination of the features of two different models, namely the Eddy Dissipation model (ED) and Finite Rate Chemistry (FRC) model.

The Eddy Dissipation model is based on the concept that chemical reaction is fast compared to the transport processes in the flow. When reactants mix, the product is 
instantaneously formed. The model assumes that the reaction rate is related directly to the time required to mix reactants at the molecular level. In turbulent flows, this mixing time is dominated by the eddy properties and therefore, the rate is proportional to a mixing time defined by the turbulent kinetic energy, $k$, and dissipation $\varepsilon$, i.e. rate $\propto \frac{\varepsilon}{k}$. This concept of reaction control is applicable in many industrial combustion problems where reaction rates are fast compared to reactant mixing rates.

The Finite Rate Chemistry model uses the Arrhenius law to calculate the chemical reaction rate. This allows to compute reaction rates described by the molecular interaction between the components in the fluid. Usually the FRC model is combined with the ED approach for flames, with chemical reaction rates slow compared to the reactant mixing rate.

In ED/FRC model, the reaction rate is limited by turbulence quantities or by mixing. Therefore it can be used for calculations in which reaction rates are limited by turbulent mixing in one area of the domain and limited by kinetics somewhere else (CFX Ansys 11, 2007). Combining the ED and FRC approach allows the ED/FRC model to capture the whole range of Damköhler numbers in the reacting flow. Depending on the local Damköhler number defined as: $D a=\frac{\tau_{t}}{\tau_{c}}$, the Eddy Dissipation model ( $\left.\mathrm{Da}>1\right)$ or Finite Rate Chemistry model $(\mathrm{Da}<1)$ is applied. The effective reaction rate, for the combined model, is computed to be the minimum of the Finite Chemistry Rate and the Eddy Dissipation rate. This procedure is applied for each reaction step separately, so while the rate for one step may be limited by the chemical kinetics, some other step might be limited by turbulent mixing at the same time and physical location. The whole range of methane-air flames starting from lean and ending in stoichiometric regime can be calculated with the use of ED/FRC model.

Next to the relatively simple combustion model, the UT in-house combustion model called CFI is tested. However, because of the high grid resolution and time step demands, the CFI model is used only for the steady-state calculations. Thus no information about acoustic wave propagation was obtained and furthermore the model was not included in the fluidstructure calculations which are the scope of this thesis. The CFI model is described in detail by (Derksen, 2005), (Jager, 2007), (Pater, 2007). Results of calculations using the CFI model and a short description of the model itself are presented in Appendix D.

\subsection{Aero-ACOustics}

The acoustic phenomenon in the gas turbine combustors originates from different sources. Vibrating mechanical structures, regions of turbulent flow, mixing of fluids with 
different temperature are only a few examples of sound generation mechanisms. The first one to identify and classify those mechanisms was Sir James Lighthill in the early fifties, see (Lighthill, 1952), (Lighthill, 1954). He distinguished three main acoustic sources which exist in the fluid flow, namely the monopole, dipole and quadrupole source. Because the acoustic wave is generated in a flowing medium, the name of aero-acoustics was accepted to describe the new branch of science. The three acoustic sources were derived from the mass conservation law and the momentum equation. First, the time derivative of the mass conservation equation, see Equation 3-1, and the divergence of the momentum equation, see Equation 3-2, as it is shown on Equation 3-34 and Equation 3-35, are taken.

$$
\begin{gathered}
\frac{\partial^{2} \rho}{\partial t^{2}}+\frac{\partial^{2} \rho v_{i}}{\partial t \partial x_{i}}=0 \\
\frac{\partial^{2} \rho v_{i}}{\partial t \partial x_{i}}+\frac{\partial^{2} \rho v_{i} v_{j}}{\partial x_{i} \partial x_{j}}=-\frac{\partial^{2} P_{i j}}{\partial x_{i} \partial x_{j}}+\frac{\partial f_{i}}{\partial x_{i}}
\end{gathered}
$$

Then, Equation 3-35 is substituted from Equation 3-34. To obtain the acoustic wave equation, to both sides of the outcome equation the term $\frac{1}{c_{o}^{2}} \frac{\partial^{2} p}{\partial t^{2}}$ is added. After the rearrangement of the equation components and taking into account that the viscous stress tensor is expressed as shown in Equation 3-5, the non-homogenous wave equation in the form presented in Equation 3-36 is obtained. This equation is valid for any value of the velocity $c_{o}$, therefore further introduction of the perturbation (marked as "') from the reference state (marked as ${ }^{\prime}{ }^{\prime}$ ), i.e. $\rho^{\prime}=\rho-\rho_{o} ; p^{\prime}=p-p_{o}$ etc. is necessary. In a uniform, stagnant fluid this leads finally to the exact Lighthill analogy where three main sources of sound are on the right hand side (RHS) of Equation 3-37.

$$
\begin{gathered}
\frac{1}{c_{o}^{2}} \frac{\partial^{2} p}{\partial t^{2}}-\frac{\partial^{2} p}{\partial x_{i}^{2}}=\frac{1}{c_{o}^{2}} \frac{\partial^{2} p}{\partial t^{2}}-\frac{\partial^{2} \rho}{\partial t^{2}}-\frac{\partial f_{i}}{\partial x_{i}}+\frac{\partial^{2}\left(\rho v_{i} v_{j}-\tau_{i j}\right)}{\partial x_{j} \partial x_{i}} \\
\frac{1}{c_{o}^{2}} \frac{\partial^{2} p^{\prime}}{\partial t^{2}}-\frac{\partial^{2} p^{\prime}}{\partial x_{i}^{2}}=\frac{\partial^{2}}{\partial t^{2}}\left(\frac{p^{\prime}}{c_{o}^{2}}-\rho^{\prime}\right)-\frac{\partial f_{i}}{\partial x_{i}}+\frac{\partial^{2}\left(\rho v_{i} v_{j}-\tau_{i j}\right)}{\partial x_{j} \partial x_{i}}
\end{gathered}
$$


The first term in Equation 3-37: $\frac{\partial^{2}}{\partial t^{2}}\left(\frac{p^{\prime}}{c_{o}^{2}}-\rho^{\prime}\right)$ corresponds to a monopole source. This type of acoustics source radiates acoustics energy equally well in all directions. Therefore it behaves like a pulsating sphere, i.e. expanding and shrinking with various frequencies. The acoustic energy radiated by a monopole source is presented on Figure 3-2 (left). In the combustion chamber a flame is a monopole acoustic source. Depending on the temporary flame positions and wrinkling, hot gases expand or contract in each direction simultaneously.
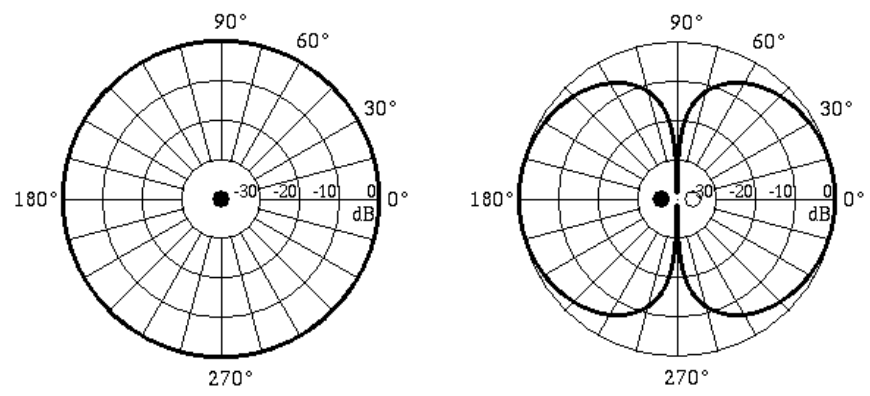

Figure 3-2: Acoustic monopole (left) and dipole (right); pictures taken from (Russell, 2008)

A dipole source is represented by term $\frac{\partial f_{i}}{\partial x_{i}}$. This source radiates energy well only in two directions and in two other no sound is emitted, as it is shown in Figure 3-2 (right). The source consists of two monopole sources separated by a small distance from each other with respect to the wave length. Both monopoles have the same strength but they are opposite in phase, therefore when one "sphere" is expanding the other is shrinking. An oscillating back and forth sphere is an example of a dipole source. In the combustion chamber a dipole source can be induced by an external force field. This non-uniform force exerted on the gas inside the combustion chamber originates mainly from the vibrating walls.

The last term: $\frac{\partial^{2}\left(\rho v_{i} v_{j}\right)}{\partial x_{j} \partial x_{i}}$ in Equation 3-37 is a quadrupole source. Two dipoles opposite in phase make a quadrupole source. Depending on the localization of those dipoles, a lateral or linear quadrupole source can be distinguished. Both types of quadrupole source differ with respect to the sound radiation. The lateral source radiates well sound in the front of each monopole source, but there are places where sound is cancelled (see directivity pattern presented on Figure 3-3 left). The linear source radiates well sound along the line where 
individual monopoles are placed and less well in the direction perpendicular to this line. Similar to the lateral source, the sound is not radiated equally well around the source, and there are some places where sound is not present (see Figure 3-3 right). The turbulence field inside the combustion is generated by the second order gradient of the velocity and therefore acts as a quadrupole source. The viscous stress tensor $\tau_{\mathrm{ij}}$ present in Equation 3-37 can be neglected due to the high Reynolds number at which the combustion chamber operates.
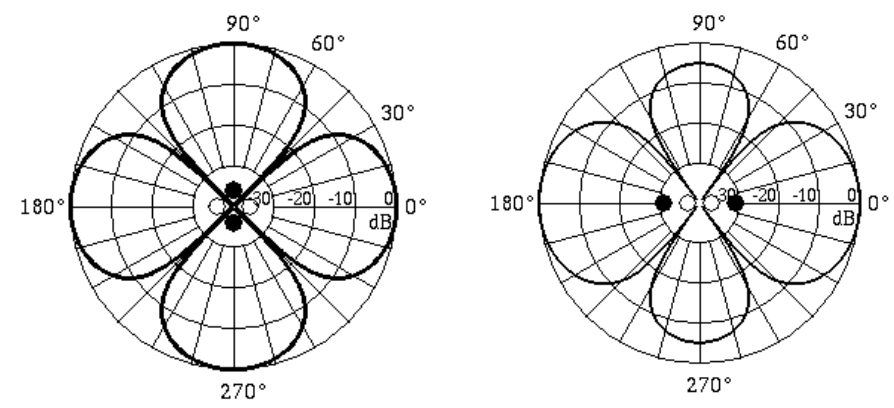

Figure 3-3: Different types of quadrupole source: lateral (left) and linear (right); pictures taken from (Russell, 2008)

The analysis performed on the different types of sources in the combustion chamber, see (Klein, 2000) shows that the acoustic power from the combustion process (monopole sound) is the strongest acoustic source. It is bigger by about the order of $1 / \mathrm{Ma}^{4}$ (here $M a \cong 0.1$ ) than the acoustic power generated by the unsteady turbulence field (quadrupole source). The quadrupole source can be neglected, because during the combustion process, the device in order to keep pressure loss induced by high speed flows low, is operating on low Mach number, as presented in (Lefebvre, 1983). The effect of the dipole source on the acoustic field in the combustion chamber strongly depends on the stiffness of the walls and it is described in the remaining chapters of this thesis.

\subsection{Combustion Noise}

The acoustic equation (Equation 3-37) described above is valid for the medium in rest. The equation is derived directly from the Navier-Stokes equation, thus the effect of the flow is incorporated in the equation in the form of sources which move through the stagnant 
fluid. A more appropriate way to describe the flame in the combustion chamber seems to be the acoustic equation which includes convection due to the flow and stagnant source. Especially because the movement of the flame is considered small here compared to the acoustic wavelength observed in the chamber. The homogenous wave equation in the convective form is presented in Equation 3-38. This equation does not include any acoustic source thus is valid for regions far from the acoustic source. When a thermo-acoustic source, i.e. a flame, is included and other sources of acoustic wave are neglected (due to low Mach number), the non-homogenous convective acoustic equation in the form of Equation 3-39 is obtained.

$$
\begin{gathered}
\frac{1}{c_{o}{ }^{2}} \frac{D^{2} p^{\prime}}{\partial t^{2}}-\frac{\partial^{2} p^{\prime}}{\partial x_{i}{ }^{2}}=0 \\
\frac{1}{c_{o}{ }^{2}} \frac{D^{2} p^{\prime}}{\partial t^{2}}-\frac{\partial^{2} p^{\prime}}{\partial x_{i}{ }^{2}}=\frac{\partial}{\partial t}\left[\frac{\gamma-1}{c_{0}^{2}} q_{f}^{\prime}\right]
\end{gathered}
$$

Where: $q_{f}^{\prime}$ is the heat release rate in $\mathrm{W} / \mathrm{m}^{3}$ and it is proportional to the acoustic mass flow source via relation: $m_{s}^{\prime}=\frac{\gamma-1}{c_{0}^{2}} q_{f}^{\prime}$. The factor $\frac{\gamma-1}{c_{0}^{2}}$ is assumed to be constant and it can be evaluated at adiabatic flame temperature, see (Van Kampen, 2006). For the high Reynolds number flows, the main term which leads to the pressure fluctuations is the fluctuation of temperature due to formation of reactants and products. The other terms associated with heat conduction, i.e. viscous heating and laminar diffusion, can be neglected. Rewriting the heat release rate perturbations as a function of temperature variations, see (Van Kampen, 2006), leads finally to Equation 3-40.

$$
q_{f}^{\prime}=-\rho c_{p} \frac{D T^{\prime}}{D t}
$$

As an effect of flame temperature perturbations, the flame acts as an acoustic source.

The flame can produce two types of combustion noise. The first one is called combustion roar and it takes place when the flame acts as an autonomous source of sound. In this case fluctuations in heat released by the flame are independent of the acoustic pressure fluctuations inside the combustion chamber. Random fluctuations, local changes of equivalence ratio or vortex shedding are only a few of the mechanisms responsible for combustion roar. However, combustion roar has a minor influence on the combustion 
performance. More dangerous is the second mechanism of noise production by the flame, namely the mutual influence between the acoustic pressure fluctuations inside the combustion chamber and fluctuations in heat release. This type of the flame interaction is well-known as combustion instability and it is briefly discussed in Chapter 1 of this thesis.

Focus of this thesis is placed on the combustion instability however combustion roar is of course always present during experiment and numerical analyses.

\subsection{One-Dimensional ACOUSTICS}

The noise produced by the flame propagates in the combustion chamber. Due to the small characteristic diameter of the investigated duct in relation to the length of the duct and to the acoustic wave length, the acoustic field in the combustion chamber typically has a one dimensional pattern for the low frequency phenomena explored here. The general solution of the one-dimensional version of the convective, homogenous wave equation (see Equation 3-38) is presented in Equation 3-41.

$$
p^{\prime}(x, t)=A^{+}\left(x-\left(c_{0}+u_{0}\right) \cdot t\right)+A^{-}\left(x+\left(c_{0}-u_{0}\right) \cdot t\right)
$$

Equation 3-41 describes the propagation of two acoustic waves. One is travelling upstream (i.e. negative $x$ direction) with velocity of $\mathrm{c}_{0}-\mathrm{u}_{0}$ and the other downstream with velocity of $\mathrm{c}_{0}+\mathrm{u}_{0}$, as shown on Figure 3-4. The initial and boundary conditions give information about amplitudes $A^{+}$and $A^{-}$.

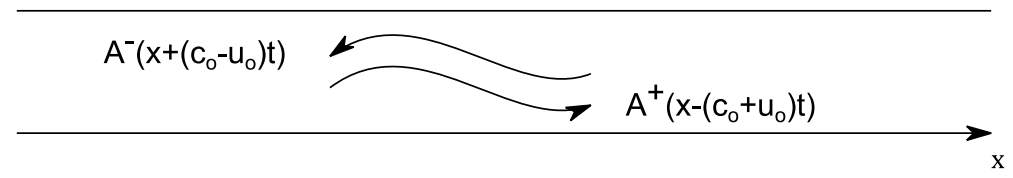

Figure 3-4: One-dimensional waves in a duct

The assumption that waves are planar is valid for all frequencies below a cut-off frequency $f_{C}$. The cut-off frequency is the lowest frequency at which transverse resonance can occur. In case of the square cross-section channel like the one investigated here, the cut-off 
frequency is determined from relation: $f_{c}=\frac{c_{0}}{2 d}$, as shown in (Rienstra \& Hirschberg, 2001). Where: $d$ is a cross sectional dimension of the square tube.

\subsection{Numerical Model}

The main target of this work is to describe the mutual interaction between combustion, acoustics and structural vibrations inside the combustion chamber. To investigate simultaneously the interaction between those phenomena, the numerical grid applied on the combustion flow domain is used for all interaction analyses. It is important to emphasise that the CFD computations are always the first step in each of the coupling investigations shown here. Therefore, on one hand the numerical grid resolution must be dense enough to capture flame features, but on the other hand, because coupling CFD and CSD is a very time-consuming process, the computations cannot take too long. To save computational time and at the same time to increase the number density of elements within the calculated model, the computational domain is reduced to a quarter section of the real combustion chamber, with periodic boundary conditions. A total number of 720000 unstructured elements is used for the calculations. The investigated flame is stabilized by two recirculation zones, an outer and inner. To capture the flame properties, both recirculation regions should be predicted accurately. Therefore most of the elements used for the numerical model are placed in the flame and recirculation region zones. Behind this region the mesh has a lower density. The near-wall region is created with the use of prism elements to avoid generating highly distorted tetrahedral elements at the face. The thickness of the first prism element is equal to $3 \mathrm{~mm}$. The velocity and turbulence profiles at the inlet of the combustion chamber are taken from steady-state calculations of the geometry including the pressure decouplers and plenum area. This geometry is presented in Figure 3-5 (left), whereas in Figure 3-5 (right) the reduced computational domain used for combustible flow calculations is shown. 

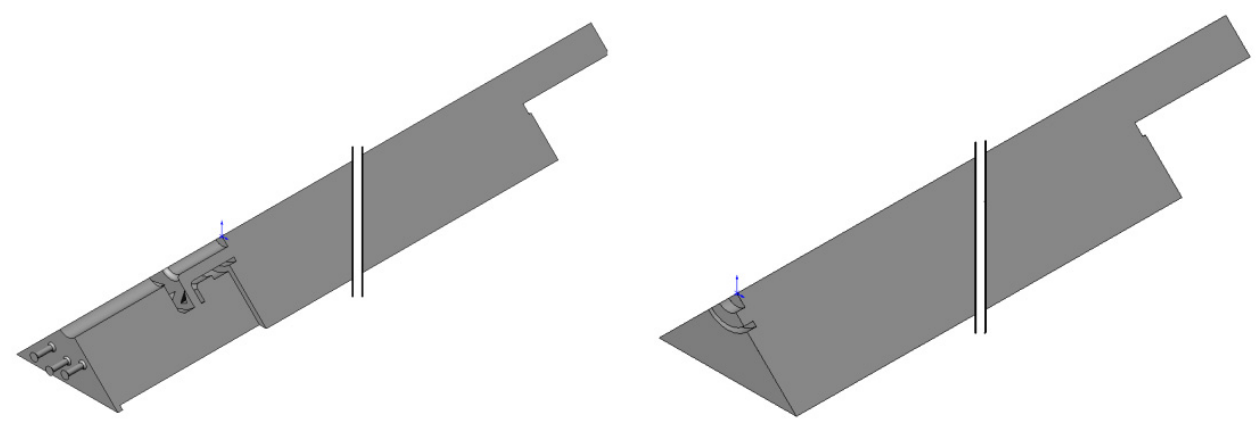

Figure 3-5: Computational: full setup geometry (left) and reduced geometry (right)

The calculations of the full geometry are done only for the isothermal case (without combustion). The results of the isothermal calculations are exported later as the inlet boundary conditions to the reacting flow model which includes only the combustion chamber. A static average pressure is imposed at the combustion chamber outlet. For the transient combustion calculations, forced oscillations are investigated. In this case, the fuel to air equivalence ratio is oscillating with frequency $300 \mathrm{~Hz}$ and amplitude $8.5 \%$ of the mean. On the wall, where structural vibrations are investigated, a heat transfer coefficient is imposed. Other walls have adiabatic no-slip boundary conditions. The influence of the pressure fluctuations in the cooling passage are neglected because measurements have shown these to be at least $6 \mathrm{~dB}$ less than in the combustor (see Chapter 2). The investigated flame is the premixed natural gas flame under conditions presented in Table 2-2. If it is not mentioned otherwise, the mesh and boundary conditions described in this section are used for the calculations.

Several factors have a crucial impact on the accuracy of the numerical calculations. The influence of the adiabatic and non-adiabatic walls, turbulence and combustion model as well as CFL number are presented in the following part of this chapter.

\subsection{Steady-State Calculations}

The results of steady-state computations of the combustible flow are compared to the experimental data. Computed during steady-state calculation heat transfer coefficients, velocity and turbulence profiles are used as boundary conditions in the transient investigation. 
The k- $\varepsilon$ turbulence model is used for the calculations. This model provides a sufficiently well approximation of the recirculation region and flow properties, see (Pozarlik, Panara, Kok, \& van der Meer, 2008), and it is not time consuming. The Eddy Dissipation/Finite Rate Chemistry and CFI combustion model are used both for the steady-state calculations.

\subsubsection{Adiabatic vs Non-Adiabatic Wall}

The acoustic phenomena strongly depend on the combustion induced temperature field inside the combustion chamber. To predict the (hazardous) frequencies at which instabilities occur, the temperature distribution inside the combustion chamber has to be known. To calculate the temperature profile, the heat transfer coefficient instead of the adiabatic condition must be imposed on the investigated wall. To get information about the heat transfer coefficient, the model of the combustible flow, described above (and presented in Figure 3-5), is modified. The geometry composed of the combustion chamber together with the liner wall and cooling passage is investigated. The ratio, between two mass flow rates: the cooling air in the cooling passage and the combustible mixture in the combustion chamber is set to 1 . The outer wall of the cooling passage is adiabatic. The heat transferred to the environment and any radiation from the combustion chamber, are not included in the calculations. The cooling and combustible flows are separated by a solid region - the liner wall. The solid region was created in the CFX code, and its properties are those of stainless steel. The heat transfer equation for the liner wall is simplified because there is no flow in the solid domain. Heat transfer is present only as conduction. Thus a conjugated heat transfer problem is solved. The grid size is changed, however; like in the main case described above, the elements are non-uniformly distributed over the entire domain. Most of the elements are placed in the flame and recirculation regions. Other parameters of the flow and boundary conditions are unchanged.

The results, for the temperature at the central axis obtained from the investigation of the case with and without cooling of the chamber and liner wall, differ significantly, as shown in Figure 3-6 and in Figure 3-7 (for convenience both pictures in Figure 3-7 have the same legend scale). 


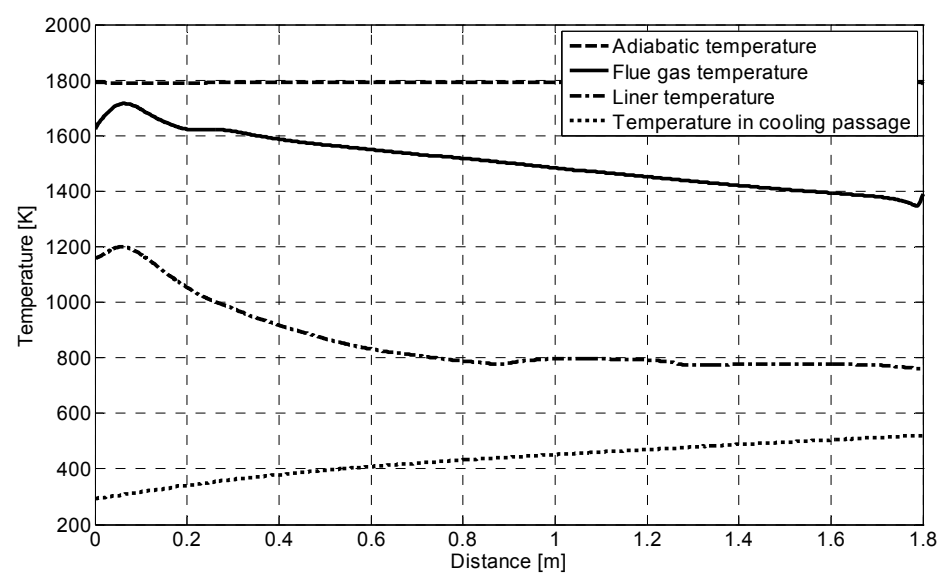

Figure 3-6: Temperature profiles in the adiabatic and non-adiabatic model

The adiabatic model shows a uniform temperature distribution along the combustion chamber with exception of the near-flame region. Sound waves predicted by this model would travel with almost constant high speed through the chamber.

The non-adiabatic model presents a maximum temperature at the flame location, followed by a substantial decrease with distance. The cooling flow reduces the temperature in the combustion chamber significantly. This reduction is almost linear from the flame region to the outlet of the chamber. The difference in temperature magnitude between investigated adiabatic and non-adiabatic model is depending on the location. The highest difference is at the end of the chamber, approximately $400 \mathrm{~K}$. The smallest difference is next to the flame region and it is equal to $50 \mathrm{~K}$, as shown in Figure 3-6. In the non-adiabatic model itself, variations in temperature at both ends of the combustion chamber reach $350 \mathrm{~K}$. These high temperature changes influence significantly the speed of sound in the chamber and as a consequence they have impact on the instabilities which occur there.

The temperature predicted by the applied model at location close to the exit from the combustion chamber is equal to approximately $1350 \mathrm{~K}$. The temperature measured at the same location during the experiment was equal to $1250 \mathrm{~K}$. In the cooling passage, the temperature is rising with distance from the flame. The maximal temperature observed near the outlet from the cooling passage does not exceed $520 \mathrm{~K}$ and it is about $30 \mathrm{~K}$ smaller than the one observed during experiment. The differences between numerical and experimental data have a source in the assumption of the adiabatic walls of the cooling passage, as well as, in neglecting radiation in the investigated numerical model. 


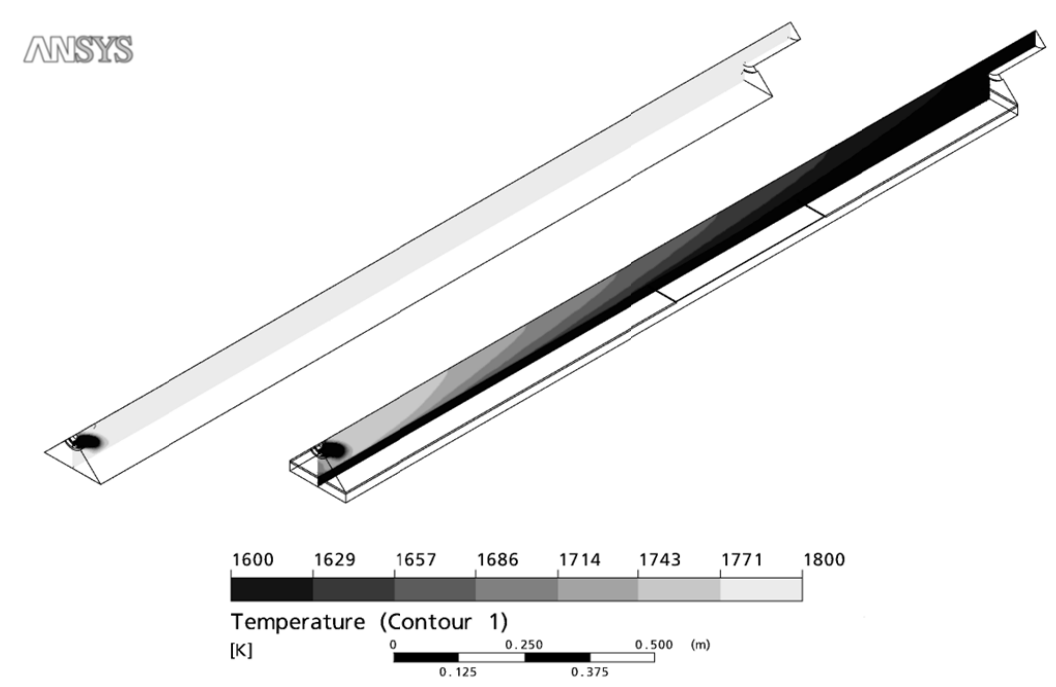

Figure 3-7: Comparison of the results for the model with adiabatic walls (left) and with nonadiabatic conditions (right)

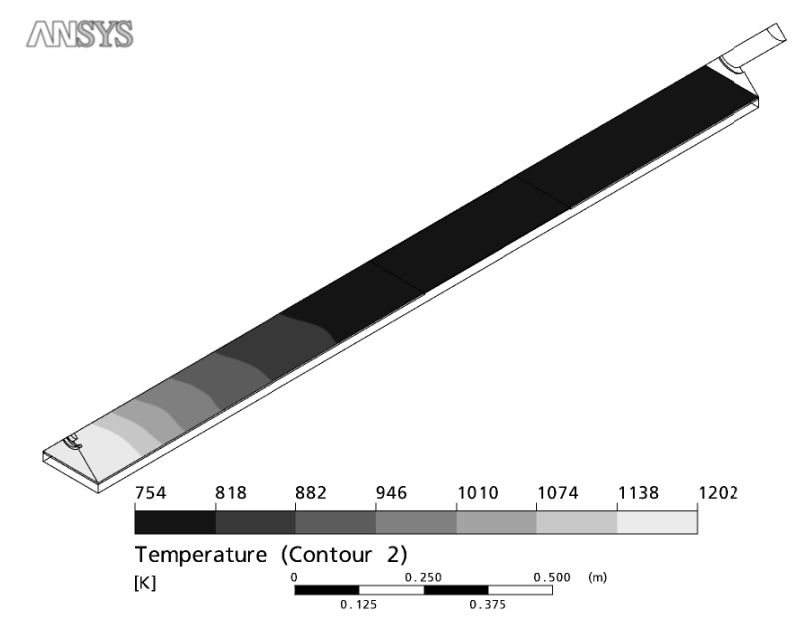

Figure 3-8: Wall temperature during steady-state calculations

The temperature distribution over the liner length is presented in Figure 3-6 and Figure 3-8. This information is very important for the next fluid-structure interaction analysis, because the temperature profile of the liner and the average liner temperature are obtained. Moreover, the flexible section of the liner is directly responsible for the structural 
calculation, because any changes in the temperature have influence on the material properties of the liner as well as on the amplitude and frequency of vibrations. Similarly to the temperature distribution inside the combustion chamber and cooling passage, the liner temperature also is varying significantly. However, the melting point of the stainless steel, which is around $1650 \mathrm{~K}$, according to (Matweb, 2008), is never reached. Two temperature bulges are visible, one at the location of flame (around $0.08 \mathrm{~m}$ from the burner) and second at the position where the liner flexible section is located $(0.9-1.3 \mathrm{~m}$ from the burner). It is also visible that the temperature profile, downstream the location $0.8 \mathrm{~m}$ from the burner, stays almost constant. The information obtained during the non-adiabatic analysis served later for the fluid-structure interaction and acousto-elastic computations. For both, the model of the combustion chamber with the heat transfer coefficient and outside temperature prescribed on the investigated wall is used.

\subsubsection{STEADY-STATE RESUltS}

The validation of the steady-state numerical results is done for a different operating point than later investigated in pulsating flow. This discrepancy was caused by the fact that this experiment was conducted earlier. To gather information about the flame during experimental tests, laser diagnostic techniques were used, as presented in (Harleman, 2005). $\mathrm{OH}$ and $\mathrm{CH}$ species were used as a marker of the flame front, see Figure 3-9. $\mathrm{CH}$ is formed during the combustion process and has a maximum concentration in the flame region. This makes this species a good indicator for the location and intensity of maximum heat release. $\mathrm{OH}$ is formed by brake down of $\mathrm{H}_{2} \mathrm{O}$ under influence of high temperature. Thus the high concentration zone of $\mathrm{OH}$ can be extended to exhaust gases from the flame. As a consequence, the location of the maximal heat release is more difficult to predict. Simultaneous measurements of both species can give information about the flame front and flame propagation.

Two techniques were used for the experimental investigations, namely PLIF and chemiluminescence. More information about both techniques can be found in e.g. (Linne, 2002) and (Steijger, 1998). Molecules electronically excited from the thermal equilibrium are denoting by '*'. Comparison of the experimental data and numerical results is presented in Figure 3-9 and Figure 3-10. Unfortunately, the ED/FRC combustion model used here does not predict the $\mathrm{OH}$ and $\mathrm{CH}$ field. Therefore a comparison is done for the turbulence kinetic energy, velocity and temperature profiles instead.

The numerical results show a good agreement with experimental data. The maximal values of the kinetic energy are found at locations with high RMS and mean $\mathrm{OH}$ concentration, as 
shown in Figure 3-9 (left and middle) and Figure 3-10 (left). This indicates that indeed strong correlation between $\mathrm{OH}$ concentration and turbulence kinetic energy exists. Numerical results give also indications of a high velocity close to the wall. This velocity transports $\mathrm{OH}$ out of the flame front, thus the distribution pattern of $\mathrm{OH}$ and $\mathrm{CH}^{*}$ during the experiment varies slightly in the near-wall region. Both experimental results of $\mathrm{OH}$ and $\mathrm{CH}^{*}$ show the same opening angle of the flame. Moreover they show that the flame is mainly formed close to the inner side of the chamber. This behaviour is a consequence of the strong inner recirculation region which transports hot combustion products to the centre of the conical flame. The outer recirculation region has smaller influence on the combustion process because of the heat transfer through the wall to the cooling passage. The overall temperature in that corner of the combustion chamber is smaller. Both recirculation regions stabilize the flame. The borders of the recirculation regions can be read from the velocity vector profiles, as presented in Figure 3-10 (left).

The dominant flame formation location is marked correctly by turbulent kinetic energy. The inner profile of the $k$ field is much stronger than the outer which is in good agreement with the experimental data. However, the shape of the kinetic energy field is slightly different from the experimental one. Also the temperature profile solved by CFX shows a more uniform and shorter flame than observed during the experiment.

The under-prediction of the results is mainly caused by limitations of the combustion model used for the flame calculations. The one-step model of the combustion which uses only two reactants $\left(\mathrm{CH}_{4}\right.$ and $\left.\mathrm{O}_{2}\right)$ and products $\left(\mathrm{H}_{2} \mathrm{O}\right.$ and $\left.\mathrm{CO}_{2}\right)$ should be substituted by a more complex model. In spite of these limitations, the results obtained here show good agreement with experimental data. Therefore conditions described here were used in further investigation of the combustion instabilities that occur in the combustion chamber.
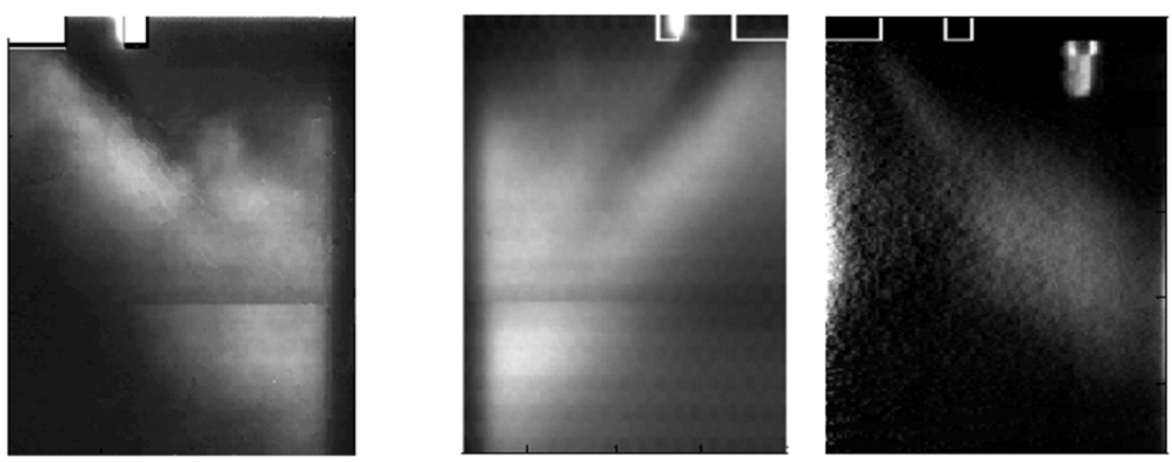

Figure 3-9: Experimental results $\mathrm{RMS}$ mean $\mathrm{OH}$ (left), mean $\mathrm{OH}$ (middle) and Abel transform of mean $\mathrm{CH}^{*}$ (right) from (Harleman, 2005) 


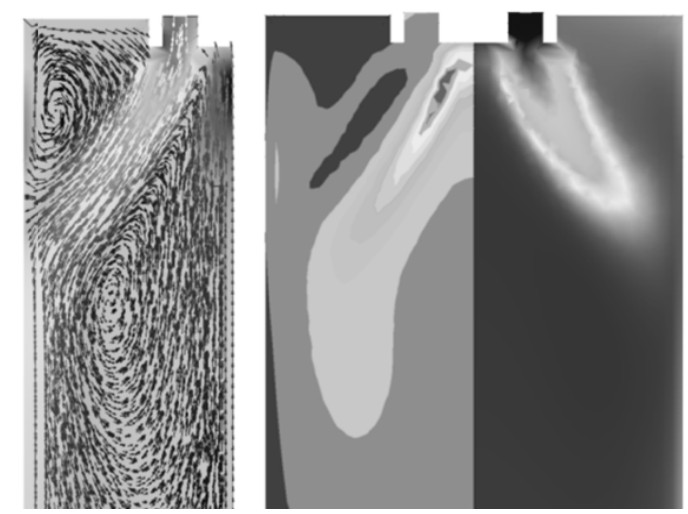

\section{aNSYS}

Figure 3-10: Steady-state numerical profiles of: axial velocity (left), turbulence kinetic energy (middle) and temperature (right)

\subsection{Transient Calculations}

In this section, the transient calculations performed with the pulsating inlet velocity are presented. In the transient line of investigation, the URANS k- $\varepsilon$ and SAS numerical approach are used for combustion flow prediction. Both models are compared with each other. The less dissipative one is used for most of the further numerical calculations. The results of pressure fluctuations during the transient flow are collected downstream in the combustion chamber, at a position corresponding to the location of the pressure sensor during the experiment. Transient investigation is done only with application of the ED/FRC model as it demands less computational effort in comparison to the CFI model.

This section is devoted to the CFD computation results only. The simultaneously investigated CSD model is presented in Chapter 4. Also, the two-way coupling model is investigated later as the CFD model includes moving mesh. The displacement of the mesh is directly related to the results of structural calculations and has influence on the investigated flow.

\subsubsection{CFL Number INFLUENCE}

The direct prediction of acoustics by the CFD computation is difficult to achieve, mainly due to requirements of meshing and time step. In most of aeroacoustics problems, large variations in the length scales of resolved structures are involved. However, as shown 
in (Polifke, Poncet, Paschereit, \& Doebbeling, 2001) and (Van Kampen, 2006), it is possible to obtain good predictions in acoustic waves propagation from the CFD calculations.

In the fluid-structure interaction in the combustion system, a high grid resolution and small time step must be used to resolve accurately both combustion and turbulent flow. Resolving accurately the compressible Navier-Stokes equations for acoustics, with such a high grid density, demands even a smaller time step. The relation between the mesh resolution and time step for acoustics is given by the Courant-Friedrichs-Lewy correlation also known as CFL number. The CFL number is defined as in Equation 3-42.

$$
C F L=\frac{(\mathrm{u}+\mathrm{c}) \Delta \mathrm{t}}{\Delta x}
$$

Where: $\Delta t$ is the time step and $\Delta x$ stands for the length interval. This number gives the stability conditions during the CFD calculations. The explicit computations are especially highly sensitive on the CFL number. In the simulation of the reacting flow in the combustion chamber where acoustics plays an important role, to resolve the acoustic wave accurately, the CFL number should be below unity. Moreover, the CFD mesh must span all the way to the reception points with enough spatial resolution to directly resolve acoustic waves over the propagation distance with minimal to no numerical damping. Those requirements make resolving acoustic directly in CFD codes very costly. The computational costs are even higher when the CFD is combined with the CSD. For the structural calculations, time step and mesh resolution can be bigger than used in the CFD, however because the CFD analysis is the leading one, the time steps used for both computations is the same.

Calculations with the CFL number above unity are possible for the implicit numerical schemes (like CFX), but it entails higher uncertainties in the results. Numerical dissipation and dispersion increase together with the CFL number. They change the travelling acoustic wave in an unphysical manner. The longer the propagation distance and the higher the frequencies involved the more significant effect of those two is observed. Numerical dissipation damps the vibration amplitude and numerical dispersion changes the propagation velocity (Blom, 2003). An example of the increasing of numerical errors together with increasing time step for constant mesh resolution (thus increased CFL number) is presented in Figure 3-11. Introduction of higher than second-order methods in the CFD analysis can decrease errors made by numerical damping (Tam, 1995), but also will influence computational time. 


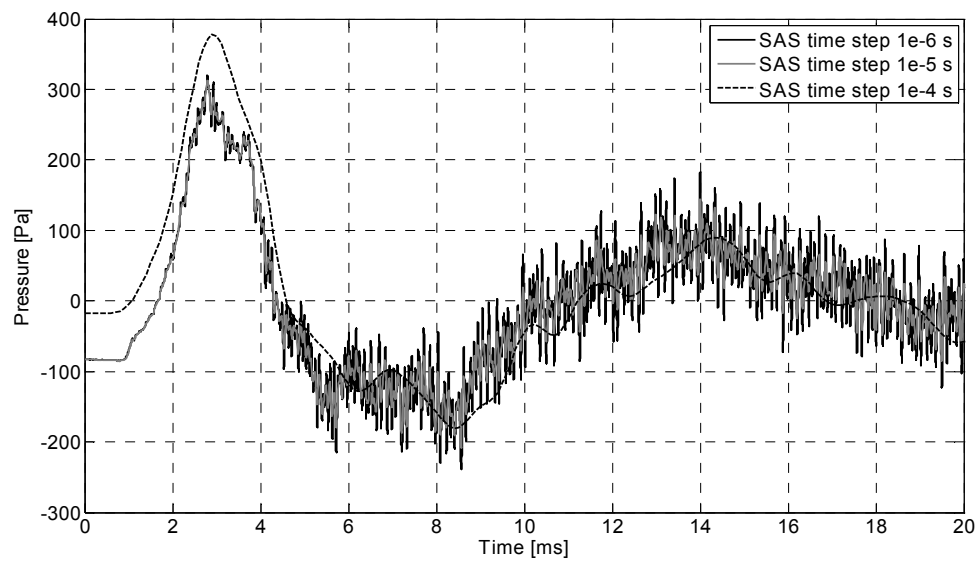

Figure 3-11: Effect of increase time step on the pressure inside the combustion chamber (here for SAS turbulence model at location P2)

To reduce the computational effort, calculations with the CFL number higher than unity are performed. For the non-adiabatic model, based on the characteristic length scale of elements ( $3 \mathrm{~mm}$ in vicinity of the wall, $3 \mathrm{~mm}$ in the flame zone and up to $8 \mathrm{~mm}$ in the rest of the investigated domain), and time step (1e-4 s), the corresponding volume average CFL number is approximately equal to 11 . The CFX code is an implicit scheme thus is stable even during calculations with high CFL number. It is expected, that the instability frequencies are not located at high frequency range, thus introduced here numerical dispersion has minor influence on the results. The numerical computations are highly time consuming therefore only 80-100 milliseconds from starting flow pulsations are calculated

\subsubsection{Turbulence Model Influence}

The model used for the fluid-structure interaction calculations must be able to capture the instabilities occurring during the combustion process in the combustion chamber. To examine this ability, two standard turbulence models available in the CFX-11 commercial code are investigated. Those models are: URANS k- $\varepsilon$ and SAS, see (CFX Ansys 11, 2007). For the research purpose, a very fine mesh and time step are used. The geometry is reduced to $1 / 8$ of the combustion chamber. The pulsations of the equivalence ratio are neglected. Although the simplifications were introduced, the calculations were still significantly long, therefore only the first $12 \mathrm{~ms}$ of the flow was simulated. 
The results received during numerical computations show that the SAS turbulence model is less dissipative for the acoustic energy than the earlier standard k- $\varepsilon$ model, as presented in Figure 3-12.

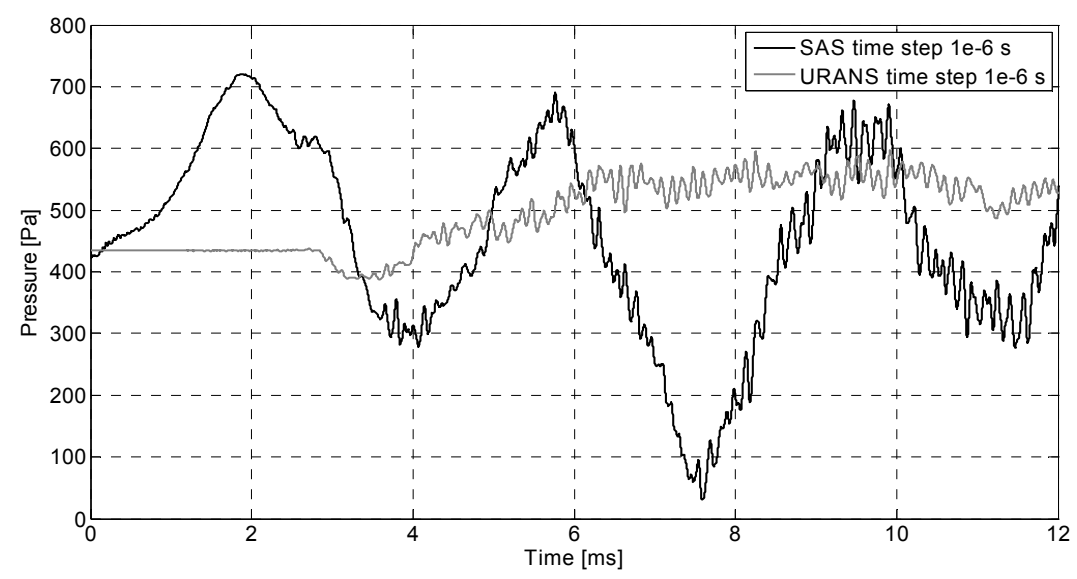

Figure 3-12: Comparison SAS-SST and URANS k-E turbulence model

This behaviour confirms observations of (Menter \& Egorov, 2005) which state that SAS can capture much better the flow instabilities than the $k-\varepsilon$ model. Therefore, most of the further analysis of mutual interaction between different phenomena during the combustion process is performed using the SAS turbulence model. Because the k- $\varepsilon$ model is widely used in industry and science, one of the fluid-structure calculations is carried out with both turbulence models. Validation of both models against the experimental data using the pulsating case is obtained this way.

\subsubsection{Numerical Data VALIDATION}

Results obtained by means of numerical computation are compared with available experimental data. The comparison is done for the unsteady pressure spectrum inside the combustion chamber. Numerical data is collected from the same location as the one during the experiment. It was already mentioned that the CFD data is also used for the fluidstructure calculations therefore to observe influence of pressure changes on the liner vibration, the numerical pressure spectrum is recorded at the location of the pressure 
transducers in the vicinity of the flexible section, i.e. P2 in Figure 2-2. Detailed data about the performed experiment is available in Chapter 2.

Based on the forcing frequency, 33 samples per period of oscillations are collected. The frequency spectrum obtained from the short calculation time (total calculation time was equal to approximately $80 \mathrm{~ms}$ ) is characterized by a relatively poor resolution due to time step and total calculation time restrictions.

The amplitudes of the maximal pressure fluctuations obtained during the experiment and during the numerical calculations show some differences, see Figure 3-13. The URANS k- $\varepsilon$ and SAS model did not capture well the amplitude of the acoustic wave propagated inside the combustion chamber. Both turbulence models present a similar pressure distribution pattern and both show a departure from harmonic behaviour. The amplitude of the predicted pressure fluctuations suggest that part of the acoustic wave is dissipated during calculations. The SAS model is less dissipative, therefore predicts higher amplitudes of pressure fluctuations with more significant departures from the harmonic behaviour compared to the k- $\varepsilon$ model. The short calculation time influenced the results because the instabilities have no time to fully develop inside the combustion chamber yet. During the experiment, the MOOG valve was switched on for few seconds after data recording was started. Information about the pressure field were collected in 10 - 20 cycles, each lasted for $3 \mathrm{~s}$, before they were averaged. This procedure assures that the pressure signal is not disturbed by other operation points which were investigated earlier. The few seconds when the MOOG valve was triggered allows also instabilities to grow up to the recorded level. Although the calculation time could have an influence on the results, it is not visible that the recorded numerical signal grows significantly with time, therefore most likely the errors are introduced by the high CFL number connected with numerical dissipation of the acoustic waves. Furthermore, the results of the numerical computations present a pressure node at location $0.87 \mathrm{~m}$ from the burner mouth, as shown in Figure 3-14. This pressure node is in the vicinity of the data recording point $\mathrm{P} 2$, thus the results from this point present only minor pressure fluctuation and lead to reduced accuracy. 

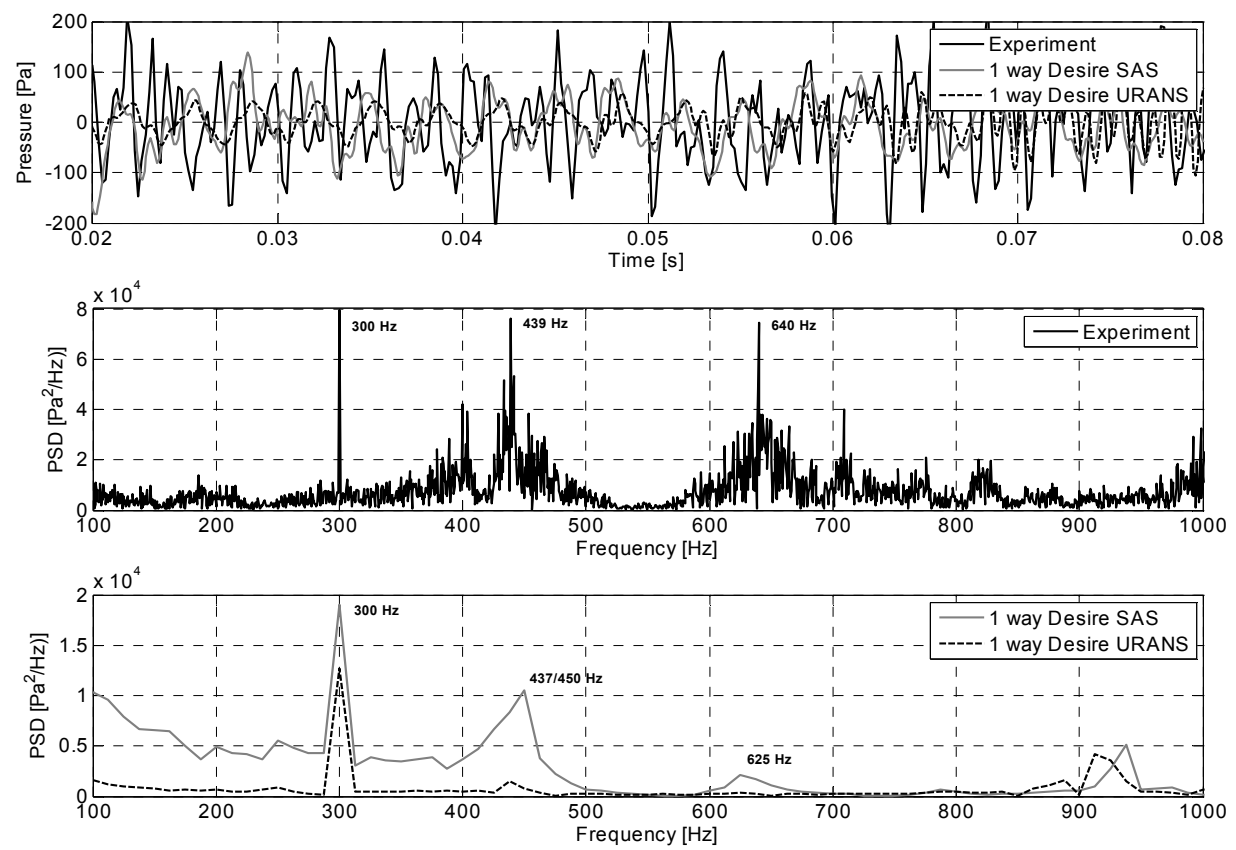

Figure 3-13: Pressure comparison at point P2

The frequency spectrum presents good agreement with the experimental data. The forcing and hazardous frequencies are predicted correctly by both turbulence models. The forcing peak at $300 \mathrm{~Hz}$ is clearly visible. For the numerical calculations this peak represents the dominant frequency which is not always a case for the experiment. The major instabilities obtained from the experimental data are located at frequency $439 \mathrm{~Hz}$. Those are also predicted accurately by the numerical computations. Similarly to the results of the pressure amplitude, also here, the SAS turbulence model presents good agreement with the experimental data. The first frequency of instability, shown by the SAS model, is located at $450 \mathrm{~Hz}$. The thermo-acoustic instability predicted by the k- $\varepsilon$ model is placed at $437 \mathrm{~Hz}$ but amplitude is much smaller than the experimental one. Instabilities visible during the experiment around $640 \mathrm{~Hz}$, are marked by the SAS model at locations $650 \mathrm{~Hz}$. The k- $\varepsilon$ model hardly presents any peak around $600-700 \mathrm{~Hz}$. 


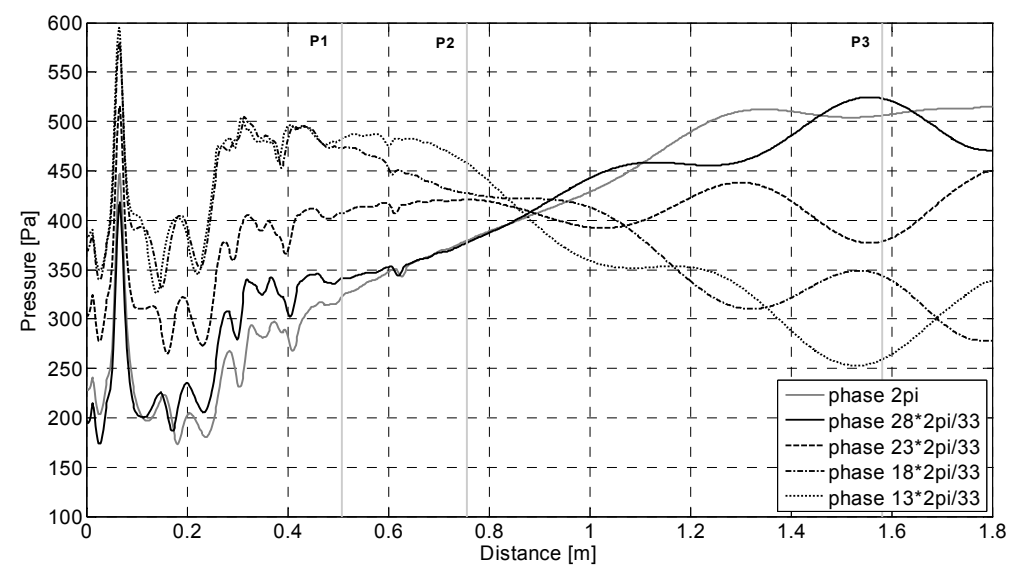

Figure 3-14: Pressure fluctuations (33 samples/period) during computations along centre line

It can be observed that the predicted frequencies of instabilities are slightly higher than those measured. This behaviour suggests that the mean temperature field inside the combustion chamber, and thus the speed of sound are slightly over-predicted with comparison to the experimental data. To improve the frequency prediction, the heat transferred not only from the combustion chamber to the cooling passage but also further on to the environment should be taken into account. Moreover, the constant heat transfer coefficient from the steady-state analysis was imposed as boundary condition of the transient analysis. Thus, the effect of the pulsating equivalence ratio on the instantaneous heat transfer is not included in the calculations.

\subsection{ConClusions}

The steady-state results were validated against chemiluminescence and PLIF data. Results show good agreement with the experimental data. The location where the flame is born, as well as the flame opening angle and recirculation regions were predicted correctly. However, the flame length predicted by the single-step combustion model is slightly shorter than observed during experiment.

The calculations using non-adiabatic wall conditions improved the numerical results. Both the temperature profiles inside the combustion chamber and the cooling passage were in good agreement with the experimental data. However, a minor over-prediction of the temperature profile was observed. This error influences also the acoustic wave propagation 
resolved during the transient calculations by moving instabilities slightly into high frequencies.

Strong influence on the numerical results by the turbulence model and numerical settings used in the computation was observed. The complex SAS-SST model was able to capture more unstable flow features than the commonly used k- $\varepsilon$ model. As a consequence results given by the SAS model were in better agreement with experimental data.

The influence of the time step and CFL number was directly related to dispersion and dissipation of the numerical wave by the numerical scheme. Both, the amplitude and frequency of the acoustic wave were damped during computations. Decreasing the time step improved results but had a huge impact on the total calculation time.

The pressure magnitudes obtained numerically present under-prediction of the results with comparison to the experiment. Numerical dissipation plays in this process the main role. Close the location of the pressure transducer P2, a pressure node was present. Although, the pressure amplitude was under-predicted, all hazardous frequencies were marked correctly. The pressure peaks around $300 \mathrm{~Hz}, 439 \mathrm{~Hz}$ and $640 \mathrm{~Hz}$ were visible in the CFD computations. This shows that for the combustion chamber configuration as presented here, the simple combustion model combined with a more complex turbulence model and relatively rough mesh was able to point out the acoustic instabilities which occur in the system. 



\section{FLUID-STRUCTURE INTERACTION}

\subsection{INTRODUCTION}

In this chapter, the mutual interaction between various phenomena occurring in the combustion chamber is investigated. Three numerical codes are combined into one calculation process to explore the coupling between natural gas combustion, acoustic wave propagation and liner vibrations inside the gas turbine combustion chamber. For the combustion process of the reacting mixture, turbulent flow and heat transfer calculations in the combustion chamber, Ansys CFX 11 is used. Acoustic waves initiated by the oscillating flame are computed by the CFD code, as well. The structural vibrations analysis is performed using Ansys Multiphysics 11. The interface connection between both codes is ensured by the MFX coupling interface code. The data is exchanged in the one-way and two-way manner using a partitioning approach. The one-way interaction method allows sending information only from the CFD code to the CSD, whereas the two-way coupling supports full information exchange between the solvers. Results of the numerical simulations are compared to available experimental data for the pressure and vibration signals.

\subsection{BACKGROUND}

The numerical simulation of the coupled problems occurs in many engineering and scientific applications, ranging from airfoil oscillations, e.g. (Guerri, Hamdouni, \& Sakout, 2008), hemodynamics, see (Penrose \& Staples, 2002), blade flutter analysis in turbomachinery, see (Sadeghi \& Liu, 2005), to power generation in the design of gas turbine combustors, presented in (Tinga, van Kampen, de Jager, \& Kok, 2007). By definition the coupled system is the system in which physically or computationally heterogeneous components interact dynamically, see (Felippa, 2004). To make numerical computations possible, the original coupled system is divided in the 
interacting computational domain fields, which are further discretized in space and time. Many interaction fields are possible, for example, fluid-structure interaction, thermal-structure interaction, electro-thermo-mechanical interaction. These are only a few types of the coupled fields which can occur in a single coupled problem. A detailed division of coupled systems can be found in (Hameyer, Driesen, De Gersem, \& Belmans, 1999). Each coupled field can be divided further with respect to numerical procedure used for solving coupled fields (monolithical and partitioned approach), direction of data transfer (one-way and two-way coupling), and physics behind the coupled processes (weak or strong coupling). The partitioned approach can be divided further on parallel and serial coupling.

\subsubsection{Monolithical vs Partitioned ApProach}

There are two main numerical approaches for simulations of the coupled system, i.e. monolithical and partitioning approach. A graphical interpretation of both, monolithical and partitioned approach is presented in Figure 4-1, where the exchange information loop between the fluid and structural code is called a stagger loop. The stagger loop can include many CFD coefficient iterations. The monolithical or socalled simultaneous treatment approach solves fluid and structure equations in a single computational domain using a single computer code. In this approach all coupled fields exchange information up to the moment the convergence is obtained. Main advantage of this method is the lack of a partitioning error which occurs when the problem is solved by two or more codes at the same time. However, usually it is necessary to developing a new code in order to capture well the interaction between all processes occurring in the investigated system. ADINA FSI is an example of a commercial code which uses the monolithical approach. However, in ADINA FSI the fluid-structure interaction calculation process is limited only to non-reacting flows, see (ADINA $R \& D, 2008)$. More information about computations using the monolithical approach can be found in (Blom, 1998), (Hron \& Turek, 2006).

The second method for solving the coupled system is the partitioned approach. In this approach the fluid and structural model are considered as isolated entities and the boundary solutions of one process are used as the boundary conditions for the other process. Separate codes can work independently and exchange information every iteration or time step up the convergence point. This allows using independent techniques to solve fluid and structure subdomains along the fluid-structure interface at a given time, see (Felippa, Park, \& Farhat, 2001), (Hermann \& Steindorf, 2003). Thus, the partitioned approach can take the full advantage of existing, well developed and 
tested codes for both, fluid and structure analysis. Furthermore, separate computations of each coupled field can be prepared by research units which are experts in different problems. To avoid degradation in stability and accuracy, the partitioned procedure requires careful formulation and implementation, see (Felippa, 2004). Drawback of this method is also the necessity of using an additional code, which provides the coupling interface between investigated fields. Nowadays, there are several commercial interface codes on the market. Examples of the most popular are MpCCI, OASIS4, FLECS and MFX. Detailed information about them can be found in (MPCCI, 2008), (OASIS4, 2008), (FLECS, 2008), (Ansys, 2007), respectively. The data exchanged between the numerical solvers through the interface connection, during the partitioning approach can be contaminated by errors (called partitioning errors). These errors are created during the interpolation and mapping procedures and should be investigated separately to examine their influence on the obtained results.
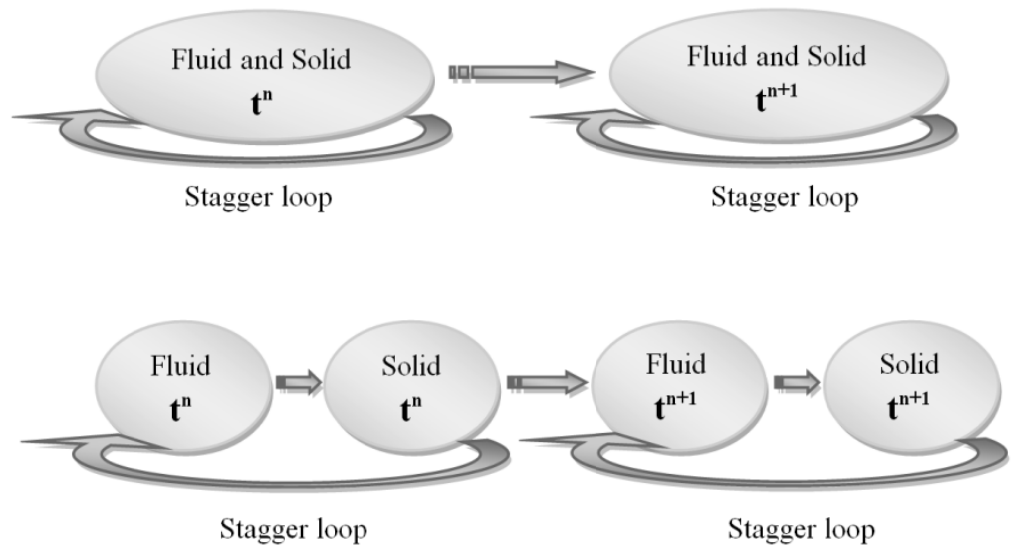

Figure 4-1: Monolithical (top) and partitioning (bottom) approach

The monolithical approach is not investigated here due to the high complexity of the problem under research and as a result of the difficulties with creating a new monolithical solver able to compute in the complex geometry interaction between turbulence, heat transfer, combustion, acoustics and vibrations. Instead, the partitioned approach was chosen. For the calculations, two commercial codes from Ansys: Ansys CFX 11 for the reacting flow calculations and Ansys Multiphysics 11 FEM package for the structural analysis are used. For convenience, later in this thesis Ansys Multiphysics 11 is called Ansys and Ansys CFX 11 is referred as CFX. Exchange of 
information between CFX and Ansys is possible with the use of the MFX coupling code, available within the Ansys FEM package.

\subsubsection{ONE-WAY VS TWO-WAY INTERACTION}

The partitioning process can be divided with respect to data exchange direction, in one-way and two-way interaction. The one-way interaction is designed for the coupled system where no feedback between interacting fields exists or the feedback is negligible due to the physics of the process. Thus during the numerical calculations, the data is sent only from one code to the other without reverse data transfer. An example of the one-way coupling system is a heat exchanger, where the thermal stresses arising in the solid material do not affect the flow of the liquid inside the heat exchanger.

When changes in one of the coupled fields directly influence the other field and vice versa, the one-way coupling strategy is not valid any more. For these strongly dependent mechanisms, the two-way coupling is necessary. In the two-way interaction calculations, process data is exchanged between all solvers every iteration or every prescribed time step, depending on the solution strategy. The interaction between blood flow to the heart and position of the heart valve presents a typical two-way interaction problem.

In case of gas turbine combustor technology, the coupled system involves interaction between combustion, acoustics and structural vibrations. Furthermore, the combustion process is associated with turbulent flow and heat transfer which makes the investigated system even more complicated. Heat transfer through the wall is investigated separately during steady-state calculations of the reacting flow inside the combustion chamber, see Chapter 3. Turbulent models are studied for steady and unsteady conditions using different geometries in Chapter 3 and Chapter 6. Therefore here, the influence of heat transfer and turbulence model on the coupled system is merged with the combustion process and it is considered further as the combustion process only. In this chapter, two different configurations of the combustion chamber: a stiff and flexible, are presented. For both, the one-way and two-way fluid-structure interaction analysis is performed. The idea behind the one-way and two-way interaction is presented in Figure 4-2. 

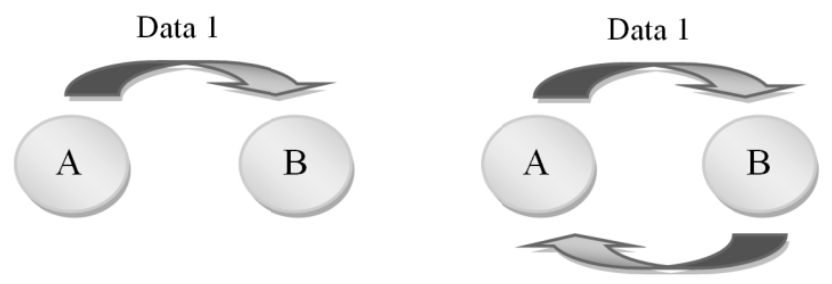

Data 2

Figure 4-2: One-way (left) and two-way (right) interaction

\subsubsection{Strong vs Weak Coupling}

The information exchange between fluid and structural solver during the partitioning approach can be done in a parallel or a serial way. The parallel method solves all equations for the flow and structural problem simultaneously, according to the scheme presented in Figure 4-3. When the iteration loop between solvers is repeated until convergence of the interacting forces, the strong coupling is achieved. The parallel strong coupling should be used to describe systems that consist of coupled fields, which cannot be solved apart, see (Hameyer, Driesen, De Gersem, \& Belmans, 1999). Significant structural displacements induced by the incompressible flow enclosed by the structure or considerable fluid mass moving together with the structure are some examples when the strong coupling algorithm should be applied (Hermann \& Steindorf, 2002), (Hubner \& Seidel, 2007).

In the serial coupling, the fluid and structural solvers work alternately as presented in Figure 4-4. All fields are solved in successive steps and the coupling is done at the end of the iteration or time step. During the call, all necessary loads are collected from one field and sent to the other solver. In case the stagger loop is done only once per few iterations or every time steps the coupling process is called a weak or loose coupling. The weak coupling is a very efficient technique, as calculation time and computer power demands are much smaller than in the case of strong coupling. The weak coupling method is applicable for coupled fields which have significant difference in density or in the characteristic timescale, see (Hubner \& Seidel, 2007).

The weak computations using a parallel method are also possible. The parallel weak coupling calculations have shorter calculation time, as solvers work independently from each other. However, this approach may also destabilize the solution process as loads obtained in less recent results are applied in each field solver (Ansys, 2007). 

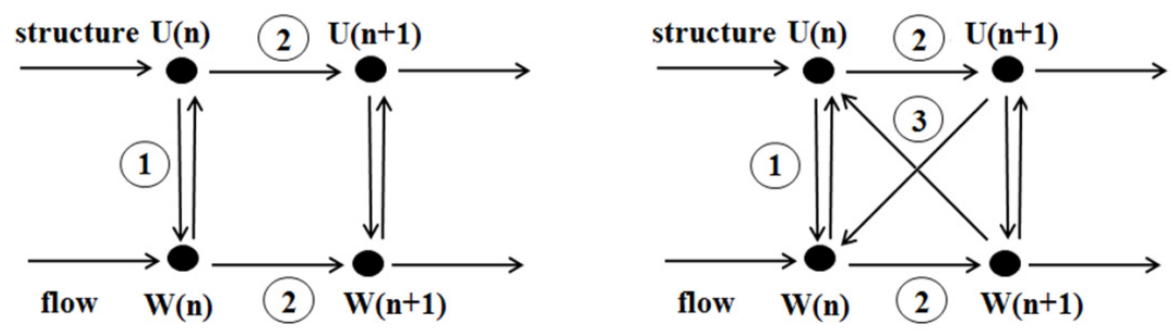

Figure 4-3: Parallel (left) and sub-iterated parallel coupling
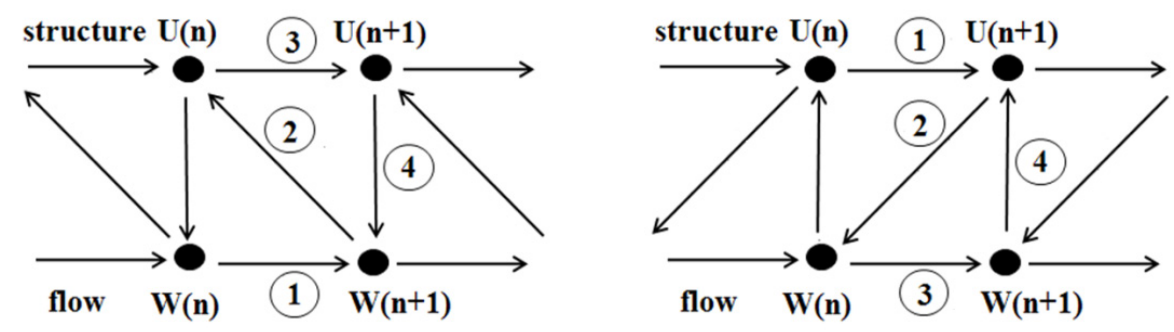

Figure 4-4: Serial $F \rightarrow S$ (left) and serial $S \rightarrow F$ coupling (right)

$U^{n}$ and $W^{n}$ are structure and fluid state at $t_{n}$, respectively.

In this work the serial $\mathrm{F} \rightarrow \mathrm{S}$ partitioned procedure as shown in Figure 4-4 (left) is performed. The problem investigated here is characterized by a significant difference in density and time scale between fluid and structural domains. The ratio between density of the hot gas and density of the hot structure is of the order of $\frac{\rho_{f}}{\rho_{s}}=\mathcal{O} \sim 10^{-5}$. The computational time step of the turbulent, combustible flow, including propagation of the acoustic wave, depends on the mesh resolution (the CFL number should be below unity). However in most of cases the time step used for turbulent flow calculations is of the order of $\mathcal{O} \sim 10^{-5} \mathrm{~s}$, whereas for the structural analyse the time step in the order of $\mathcal{O} \sim 10^{-2} \mathrm{~s}$ is sufficient. Furthermore, from experiment it is known that vibration amplitudes are small. The recorded displacement was of the order of $\mathcal{O} \sim 10^{-4} \mathrm{~m}$. This hints to the use of the weak coupling method for the numerical calculations described in this chapter.

The physical process investigated here is driven by thermo-acoustic instabilities, thus the combustible fluid flow process is resolved first. Each time step in the CFD computation consists of several iterations. When convergence is reached, the CFD 
solver sends information about the near-wall forces to the structural code and goes into a waiting phase. Up to this moment, the CSD solver was inactive. However, a signal from the CFD solver forces the FEM code to calculate the structural displacement. The calculated displacement together with its derivatives is sent back to the fluid solver via the interface which has been created earlier. In the CFD solver the changes in the geometry according to data obtained from the FEM code are performed and the next iteration step is calculated. The whole data exchange process is repeated every time step.

\subsection{LoAd Transfer Through Dissimilar Grids}

In this work, we are interested in enforcing the interface boundary condition by transferring the fluid loads to the structure and back, the mesh displacement to the fluid code, as shown in (Farath, Lesoinne, \& Le Tallec, 1998). The meshes on both sides of the interface connection are usually not matching. In most of the cases, the elements density and the elements shape of fluid and structural mesh are not coinciding spatially. Thus, robust, three dimensional matching and interpolation tools are necessary. Various methods have been developed for the problem of data transfer with nonmatching meshes. A detailed review of data transfer methods is given in (Forsythe, 2006). Two interpolation schemes are used here. Both are standard available in the FEM Ansys package. The first one is the globally conservative interpolation method, as described in (Ansys, 2007). This method is used for the one-way interaction calculations. In the globally conservative interpolation, each node on the sender side maps onto an element on the receiver side. Thus, the variable which is transferred splits into quantities that are added to the receiver nodes. This interpolation is valid when the fine mesh is on the sending side and the coarse grid on the receiver side. In the opposite situation when the finer mesh is on the receiver side and the globally conservative interpolation is performed, the load distribution on the receiver surface might not be captured well even though, the total force on the receiver is equal to the total force on the sender, as shown on Figure 4-5. 

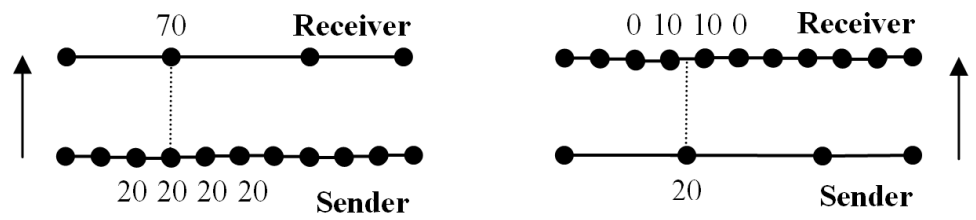

Figure 4-5: Globally conservative interpolation: fine mesh on sending side (left) and fine mesh on receiver side (right)

In the two-way interaction analysis, the data are exchanged in both directions, from the coarse mesh to the fine and vice versa. Thus, next to globally conservative interpolation, a profile preserving interpolation must be employed. The profile preserving interpolation is appropriate for the data transfer from the rough mesh model to the fine mesh model. During this interpolation, each node on the receiver side maps on an element on the sender side $\left(\alpha_{\mathrm{i}}\right)$. The transferred variable is then interpolated at $\alpha_{\mathrm{i}}$ to $\varphi\left(\alpha_{i}\right)$. Thus, all nodes on the receiver side query the sender side. The profile preserving interpolation has an advantage only during transferring data from the rough mesh to the fine grid model, as presented in Figure 4-6.
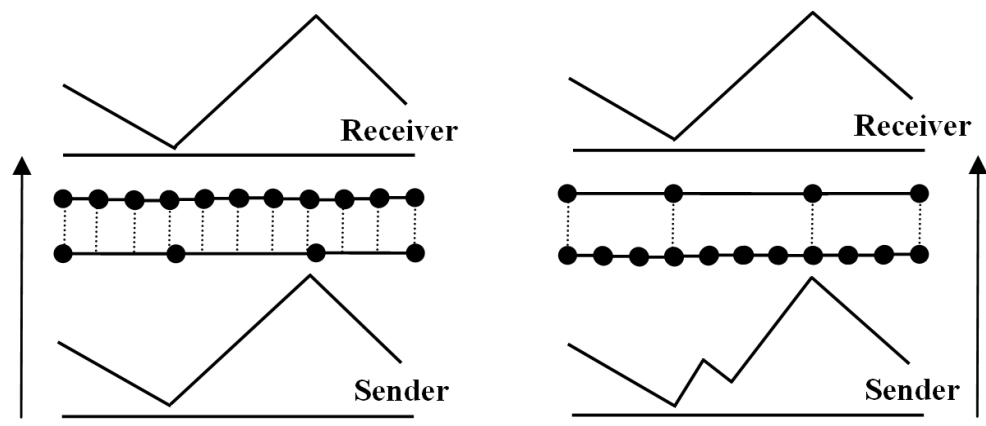

Figure 4-6: Profile preserving interpolation: fine mesh on the receiver side (left), fine mesh on the sender side (right)

In order to transfer loads across the dissimilar mesh interface, the nodes from one mesh must be mapped to an element in the other mesh. The bucket search algorithm as available in Ansys for mapping a node from one grid to element face on the other grid is used, see (Ansys, 2007). The node in question is mapped onto elements placed in Cartesian boxes. The node in question is also in the box, but it is mapped only on the 
elements in the same box. This way for a given node the algorithm restricts elements over which it loops.

To assure loss-less transfer of mechanical loads through the fluid-structure interface, the faces which share information must have the same dimensions and global coordinates. Transfer of information between faces that are separated, rotated or have significant difference in geometry is not possible. The data is transferred through the interface every time step and it includes displacement and pressure components. The information is exchanged according to the Euler equation and Cauchy stress, see Equation 4-1 and Equation 4-2, respectively.

$$
\begin{gathered}
\rho_{f} \cdot \vec{n} \cdot \ddot{\vec{u}}=-\nabla p \cdot \vec{n} \\
\vec{t}=-p \cdot \vec{n}
\end{gathered}
$$

Where $n$ is the normal direction vector, $t$ is the Cauchy stress vector in the solid, $u$ is the displacement.

\subsection{NUMERICAL MODELS}

Two numerical models, one for the combustible flow and a second for the structural vibration of the liner are created. Both models are solved using two different numerical approaches. For the flow model, the finite volume method is used, whereas the structural model is resolved using the finite element method. Detailed information about the CFD model together with the combustion model, turbulent scheme, and boundary conditions data can be found in Chapter 3. Parallel to the CFD model, the structural model of the combustion chamber wall is executed in the implicit FEM code Ansys 11. The structural model together with the boundary conditions is presented later in this chapter.

The liner of the combustion setup consists of two parts connected by a sliding connection (see Chapter 2). This configuration assures small thermal stresses during the work at elevated temperature, as both liner parts can freely expand in axial directions. The only thermal stresses come from the mutual influence of the expanding plates at the flanges and at the corners of the combustion chamber. The magnitude of these stresses is too low in any place of the liner to damage the walls, see Appendix $C$ for thermal stress calculations. Therefore, during numerical computations it is assumed that the influence of temperature 
on thermal stresses is minor. The material properties adequate to a uniform liner temperature of $760^{\circ} \mathrm{C}$ are used for calculations. Similar to the combusting flow model where only a quarter of the real combustion chamber was investigated, the structural model is reduced to one wall. The holes for pressure transducers and thermocouples, as well as the sliding connection between liner sections are located on the stiff part of the liner and their influence on the vibration amplitude is minor. Thus the investigated model is simplified to the wall without any holes, but with a flexible part located between rigid plates, see Figure 4-7.

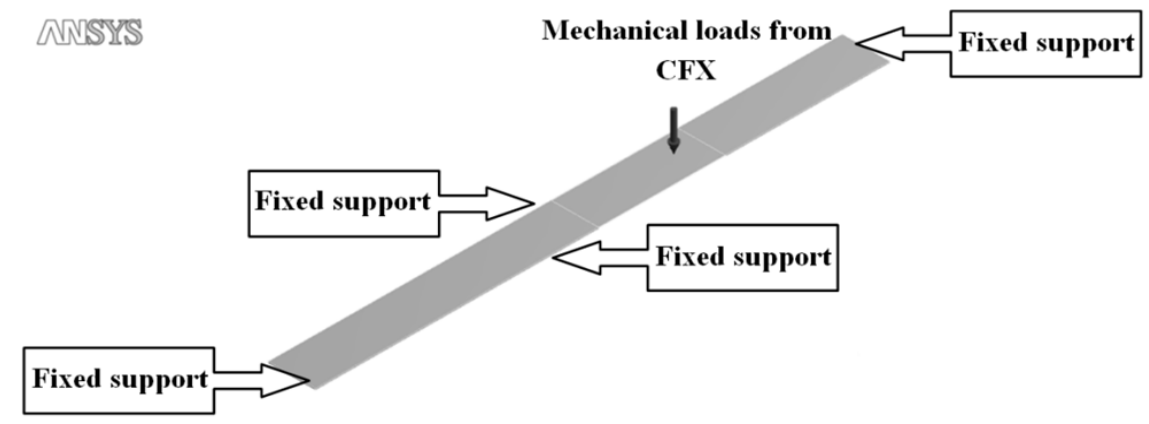

Figure 4-7: Boundary conditions used for structural analysis

Localization of the flexible part between much stiffer bodies assures well defined boundary conditions for the numerical simulation. The side parts of the liner are treated as clamped. On the inside face, the mechanical loads, i.e. pressure and shear from the CFD calculation are transferred and applied as boundary conditions, see Figure 4-7.

A total number of 19000 equally distributed 10-nodes SOLID92 tetrahedral elements, is used for the dynamic calculation. These elements have a quadratic displacement behaviour and can be used for different types of structural analyses, including plasticity, creep, swelling, stress stiffening, large deflection, and large strain capabilities, see (Ansys, 2007). In the case investigated here, the mechanical loads from the CFX calculation are transferred to the internal liner face, however, the SOLID92 elements are also capable to capture the temperature field which could be sent by the CFD code. Material properties of stainless steel 310 are adequate to conditions at elevated temperature: material density $\rho=7800 \frac{\mathrm{kg}}{\mathrm{m}^{3}}$, Young's modulus $E=138 \mathrm{GPa}$, and Poisson's ratio $v=0.3$. Two different configurations of the liner geometry are investigated, namely the Desire and the Fluistcom configuration. Geometrical dimensions of the Desire and Fluistcom configuration of the walls are 
presented in Chapter 2. The Desire configuration is characterized by higher stiffness in comparison to the Fluistcom configuration. The plate stiffness $D$ is defined according to Equation 4-3, where: $E$ is Young's modulus, $v$ is Poisson's ratio, $h$ plate thickness.

$$
D=\frac{E h^{3}}{12\left(1-v^{3}\right)}
$$

Both liner models are investigated for one-way and two-way interaction. Having two different wall configurations allows investigating the influence of the wall stiffness on the process of thermo-acoustic instabilities arising and on the overall performance of the combustion chamber. Furthermore, the influence of the interaction model on the results can be investigated. The exchange information during the coupled analysis is performed every time step. The structural and fluid field share the same time step and total calculation time. Due to the relatively short calculation time and big time step (explained in Chapter 3), the frequency sampling is around few Hz. In the current setup configuration it is impossible to measure pressure changes at the same location as the liner vibrations. Numerical results are compared with the experimental measurements at two positions: for pressure data at location of the pressure transducer $\mathrm{P} 2$, and for the liner vibrations in the middle of the flexible section (see Figure 2-2 in Chapter 2).

\subsubsection{ONE-WAY INTERACTION}

The concept of the dynamical exchange of data between the fluid and structural part through the interface connection is reduced now to one-way interaction. This means that only the pressure field from the near wall region of the CFD calculation is sent through the interface connection to the Ansys code and no information is sent back to the CFX analysis. This process is shown schematically in Figure 4-8.

Due to the significant stiffness of the Desire liner configuration it is difficult to induce a high vibration amplitude of the liner. At the same time the vibrating plate should not change significantly the acoustic pressure inside the combustion chamber. Thus, the assumption is made that the solid displacement has an insignificant effect on the fluid flow prediction. The vibration amplitude is calculated only on basis of information from the CFD program. In case of the Fluistcom liner configuration, e.g. when the liner is much more flexible, it is possible that the vibrating wall can give a feedback to the acoustic 
pressure. This possibility is further investigated by modelling it with use of both the oneway and two-way interaction.

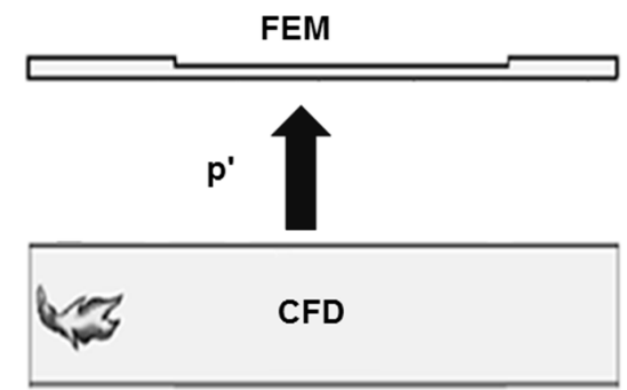

Figure 4-8: One-way interaction

\subsubsection{TWO-WAY INTERACTION}

To investigate if the assumption made for the one-way interaction is valid for the geometries presented in this thesis, also the two-way interaction analysis is performed. During the two-way interaction process, the CFX and Ansys software are forced to dynamically exchange information every time step, as shown on Figure 4-9.

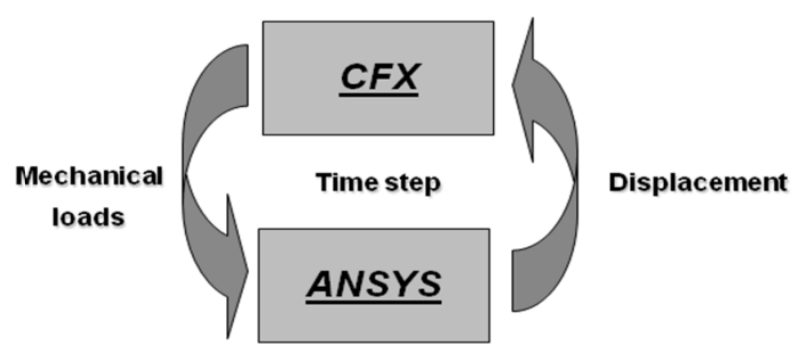

Figure 4-9: Two-way interaction

Therefore, with comparison to the one-way coupling, additional information about displacement and displacement derivatives is sent through the interface connection from Ansys to CFX. In the CFX, the nodes of the fluid grid are shifted to the position calculated in the FEM code. For the calculations without moving mesh in the CFX, the governing 
equations must be integrated over the control volume on the element. After integration and applying the Gauss Divergence Theorem to change some volume integrals into surface integrals, the mass and momentum equations can be written in the form of Equation 4-4 and Equation 4-5, respectively.

$$
\begin{gathered}
\frac{d}{d t} \int_{V} \rho d V+\int_{S} \rho u_{j} d n_{j}=0 \\
\frac{d}{d t} \int_{V} \rho u_{i} d V+\int_{S} \rho u_{i} u_{j} d n_{j}=-\int_{S} P d n_{j}+\int_{S} \mu_{e f f}\left(\frac{\partial u_{i}}{\partial x_{j}}+\frac{\partial u_{j}}{\partial x_{i}}\right) d n_{j} \\
+\int_{V} S_{u_{i}} d V
\end{gathered}
$$

However, these equations must be modified as the control volume depends on time. Therefore the term $W_{j}$ representing the velocity of the control volume boundary is introduced, see (CFX Ansys 11, 2007). After modification the resulting equations are in the form of Equation 4-6 and Equation 4-7.

$$
\begin{gathered}
\frac{d}{d t} \int_{V(t)} \rho d V+\int_{S} \rho\left(u_{j}-W_{j}\right) d n_{j}=0 \\
\frac{d}{d t} \int_{V(t)} \rho u_{i} d V+\int_{S} \rho u_{i}\left(u_{j}-W_{j}\right) d n_{j} \\
=-\int_{S} P d n_{j}+\int_{S} \mu_{e f f}\left(\frac{\partial u_{i}}{\partial x_{j}}+\frac{\partial u_{j}}{\partial x_{i}}\right) d n_{j}+\int_{V} S_{u_{i}} d V
\end{gathered}
$$

The transient term accounts for the rate of change of storage in the deforming control volume, and the advection term accounts for the net advective transport across the control volume moving boundaries, see (CFX Ansys 11, 2007). 
In Equation 4-4 to Equation 4-7, $V$ is the volume, $S$ is the surface, $d n_{j}$ are the differential Cartesian components of the outward normal surface vector, $\mu_{e f f}$ is the effective viscosity defined as $\mu_{e f f}=\mu+\mu_{t}$ where $\mu$ is the dynamic viscosity and $\mu_{t}$ the turbulent viscosity.

The regions of nodes with the same degrees of freedom are determined by the mesh stiffness according to Equation 4-8.

$$
\nabla \cdot\left(\Gamma_{\text {disp }} \nabla \delta\right)=0
$$

Where $\Gamma_{\text {disp }}$ is the mesh stiffness and $\delta$ is the displacement related to the previous mesh position.

In the calculations presented here, an uniform mesh stiffness was used and therefore the displacement is homogenously spread through the mesh. The remaining internal nodes have their position adjusted while the mesh topology is preserved. Thus, the regions with higher elements density after upgrading the mesh, according to the data from CSD, stay finer than the rest of the grid. Equation 4-8 and Equation 4-7 are solved at the start of each iteration or time step for the CFD computations, see (CFX Ansys 11, 2007).

\subsection{Results}

The results of the fluid-structure interaction are divided into the results obtained for the Desire liner configuration and for the Fluistcom geometry. Furthermore, predictions of the pressure field inside the combustion chamber and the vibration pattern of the liner wall are discussed. Because during the one-way interaction there are no forces transferred back to the CFD code the results of the pressure field inside the combustion chamber obtained using the Desire and Fluistcom liner configurations are the same. These results are presented and discussed in Chapter 3. Therefore the pressure field obtained during the oneway interaction is discussed here, only in comparison to the two-way interaction. This way the influence of the feedback loop on the system can be investigated. In case of the liner vibrations, both one-way and two-way coupling are discussed and validated against experimental data.

While coupling various calculations into one computation process, the partitioning error, which appears during data exchange through the interface, can have significant influence on the obtained results. Therefore, first the error created on the interface connection during the interpolation and mapping process is investigated. The inaccuracy is calculated during the 
two-way coupling process. Taking into account that the mesh distortion process in CFD requires more computational effort (changes in the Eulerian mesh) than forces transfer, the displacement on both side of the interface connection is compared. The numerical data, both in the CFD and FEM calculation should be the same in case the interface is constructed correctly. As can be seen on Figure 4-10 the coupling algorithm works well. Displacements on the structural and fluid face are similar. The percentage error observed during transferring data between codes is of the order of a few percent. Therefore the impact on the results is minor.

The one-way and two-way coupling is performed using a transient analysis with the CFX commercial code and dynamic calculations with the Ansys code. The result of coupling correctly these two dynamical calculations should be that the liner displacement pattern is similar not only for the first structural mode shape (the first shape in Figure 4-11) but also for the higher order modes. Thus, the current position of the liner depends not only on the last pressure data sent through the interface connection but also on the time-history of the previous liner positions. As shown in Figure 4-11, during the numerical calculations, the higher structural modes are visible. It confirms that the interaction process was coupled correctly.

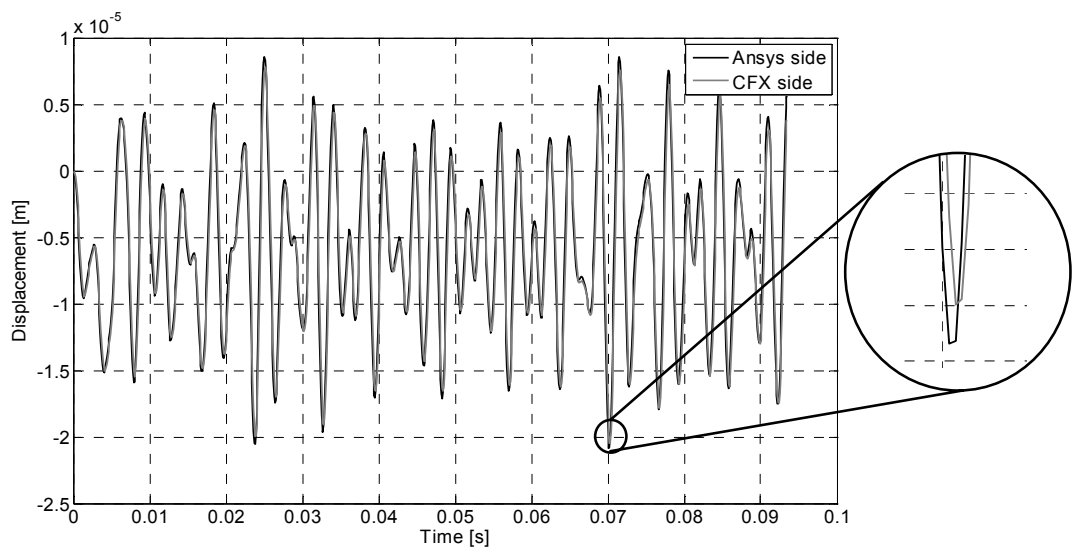

Figure 4-10: Displacements comparison on the fluid and structure side (zoomed to improve visibility of the differences) 


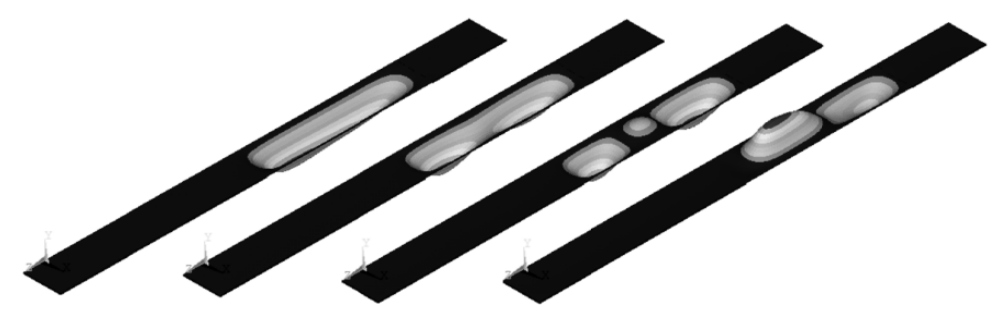

Figure 4-11: Illustration of the different structural modes observed during the one-way and two-way coupling

These results show that the coupling between the structural and fluid code is done correctly. Data is dynamically exchanged every time step and transferred between solvers with negligible errors. Therefore further analysis using one-way and two-way coupling is conducted.

\subsubsection{Desire Results}

Numerical calculations based on the Desire geometry are presented here with fuel flow forcing at $300 \mathrm{~Hz}$. These calculations are validated against available experimental data. Results of pressure fluctuations and velocity fluctuations shown in Figure 4-12 and Figure 4-13 indicate that numerical and experimental results compare well. The pressure amplitude, see Figure 4-12, is under-predicted by both numerical models, probably due to numerical damping of the acoustic waves (this problem was explained in Chapter 3). Furthermore, due to high computational costs, only $0.08-0.1 \mathrm{~s}$ of the combustion process is investigated during the numerical calculations. Within such a short time the amplitude of the acoustic instabilities does not grow up to the saturated level. 

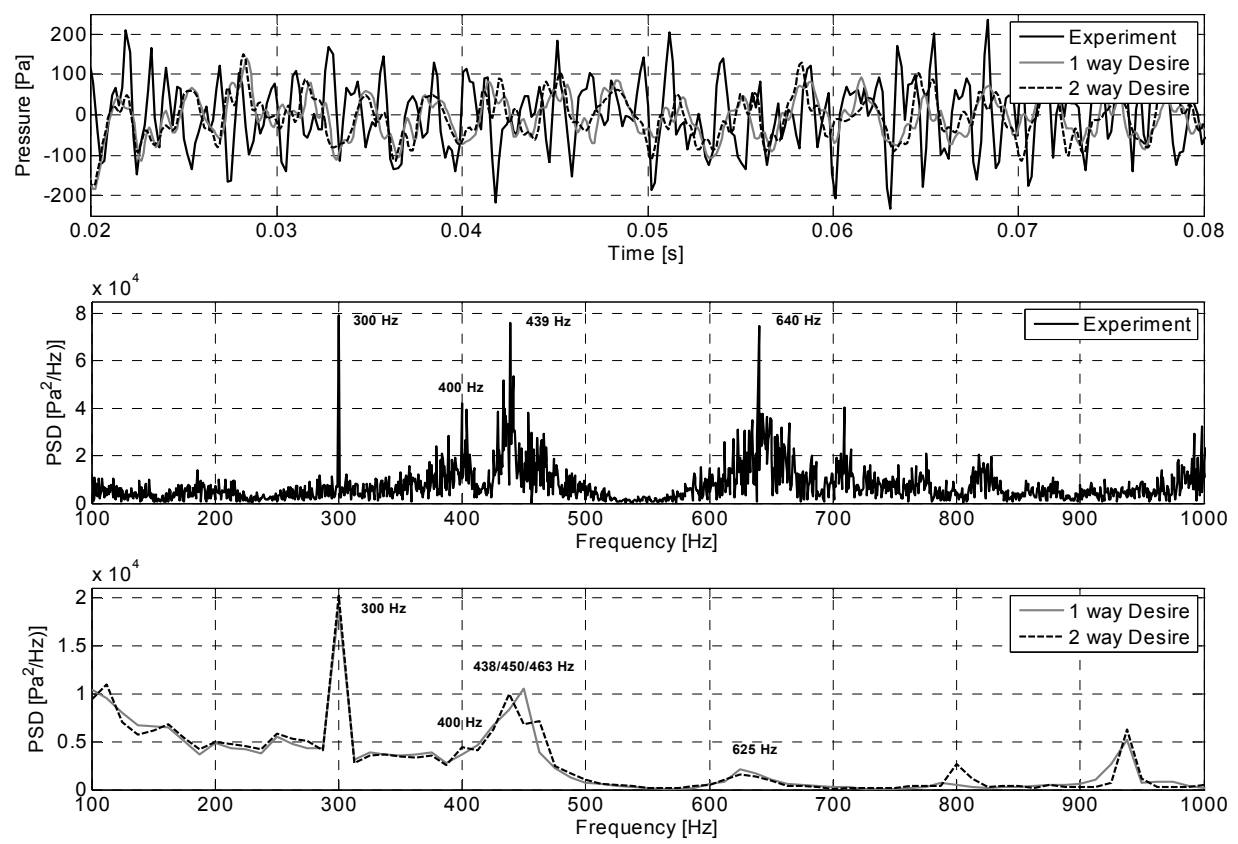

Figure 4-12: Comparison of the numerical results of the pressure signal during one-way and two-way coupling with experimental data for the Desire configuration

The two-way interaction model does not introduce significant improvement of the results. Only minor influence on the overall pressure field in the combustion chamber with comparison to the one-way interaction is observed. Both models predict the same amplitude of pressure fluctuations, which is about $43 \%$ lower than observed during the experiment (based on RMS value). However, the signal obtained from the two-way coupling includes more unstable components. This behaviour is also visible in the frequency domain, where the two-way interaction model shows additional instabilities at $400 \mathrm{~Hz}$ and $463 \mathrm{~Hz}$, compared to the one-way interaction scheme. These peaks are matched well with the experimental spectrum. The most visible instabilities observed during the experiment are at $300 \mathrm{~Hz}, 439 \mathrm{~Hz}$ and $640 \mathrm{~Hz}$. The $300 \mathrm{~Hz}$ peak is the forcing frequency the fuel mass flow is fluctuated with. The $439 \mathrm{~Hz}$ and $640 \mathrm{~Hz}$ peaks come from the thermo-acoustic instabilities arising in the combustion chamber. The first peak, slightly bigger and observed during experiments is the main instability frequency. The two-way interaction numerical results show the forcing peak at $300 \mathrm{~Hz}$ and the main instabilities at $438 \mathrm{~Hz}$. This shows that the main thermo-acoustic instability frequency was predicted correctly. Also the next peak, predicted by the numerical computations at $463 \mathrm{~Hz}$ can be recognized during the experiment as a zone where the activity of instabilities is high $(430-470 \mathrm{~Hz})$. The second 
instability frequency at $625 \mathrm{~Hz}$ is in the vicinity of experimental results $(640 \mathrm{~Hz})$. The 15 $\mathrm{Hz}$ difference in frequency can be a consequence of the temperature field obtained during the numerical calculations, as well as more significant numerical dispersion at high frequencies. Nevertheless, the prediction of the main instability frequency almost without error and the secondary instability with $2.5 \%$ error is a satisfactory result. Going to the high frequency range it can be noticed that the error between the CFD calculations and experimental results increases up to $6 \%$ as an effect of above mentioned inaccuracies. There is also a minor difference between both numerical schemes. The two-way interaction behaviour is closer to the experimental one, with similar frequencies of the instabilities. The one-way interaction also predicted well the main instabilities: the primary peak at $450 \mathrm{~Hz}$ and secondary at $625 \mathrm{~Hz}$, however some of the minor instability peaks visible in the experimental spectrum are not present in the one-way interaction analysis. The amplitude of the one-way frequency spectrum is similar to the two-way interaction. However, both numerical models under-predicted the magnitude of the instabilities, which in case of the experiment is the same as at the forcing frequency. Due to numerical dissipation and dispersion of acoustic waves during CFD computations, the main changes in the pressure field originate mostly from the changes in the main flow caused by fuel to air equivalence ratio pulsation.

In Figure 4-13 experimental and numerical results for the wall velocity are compared. Here, the velocity amplitude predicted by the numerical codes and based on the RMS value is also smaller than the experimental one. This could be explained by the under-predicted pressure amplitudes, which are the main driving force for the wall vibration. But there are additional factors that can lead to inaccuracies. For example the cooling passage, which could influence the vibrating wall, is not included in the numerical calculations. The other factor which could have influence on the smaller velocity amplitude obtained during numerical analysis is the assumption of the uniform temperature along the liner. Temperature variations change material properties and therefore affect the amplitude of vibrations. These factors are mainly responsible for smaller vibration amplitude than observed during the experiment. A comparison of results between the one-way and twoway interaction shows under-prediction of the velocity amplitude by $24 \%$ and $52 \%$, respectively. 

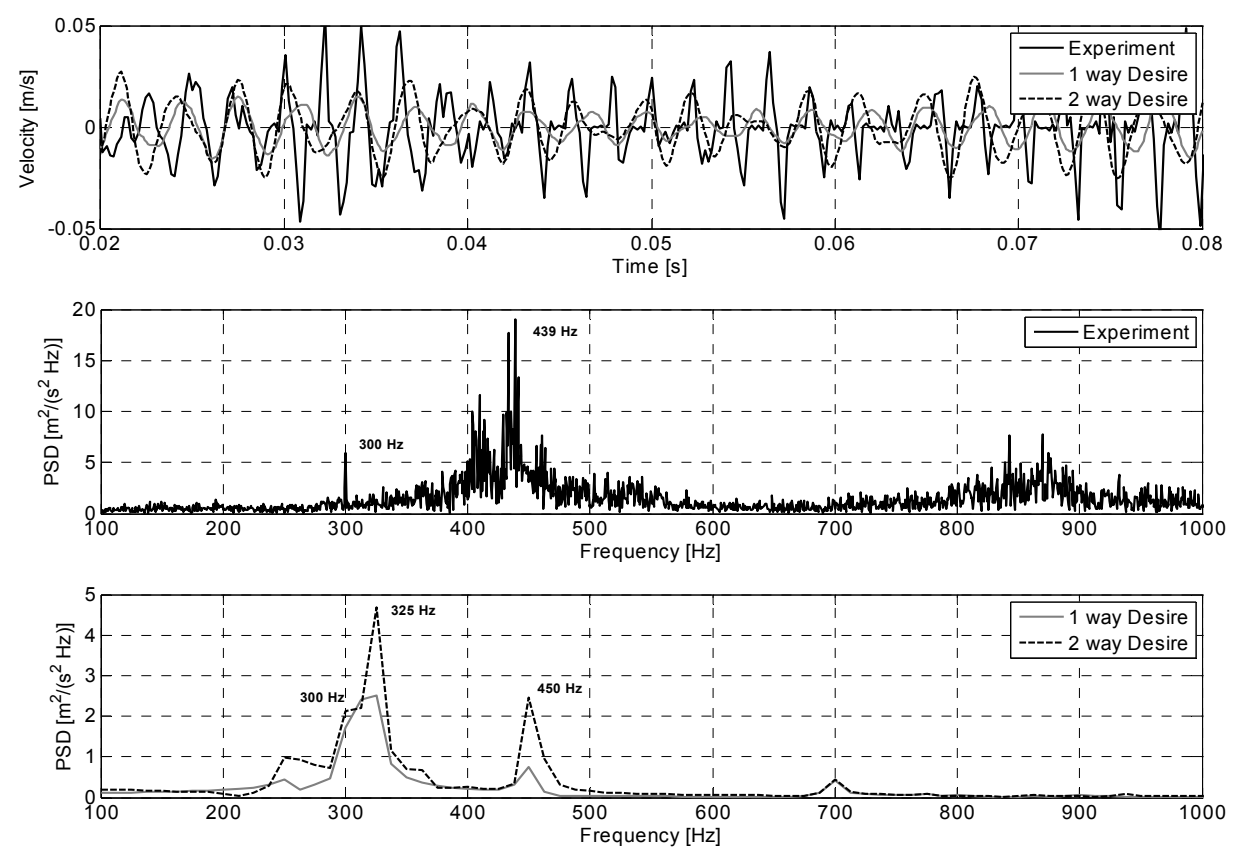

Figure 4-13: Comparison of the experimental and numerical data of the velocity signal in time and frequency domain for the Desire configuration

In the frequency domain the measured liner vibrations induced by the acoustic field show the forcing peak at $300 \mathrm{~Hz}$, and a main instability at $439 \mathrm{~Hz}$. This matches well with the frequency of pressure instabilities inside the combustion chamber. The one-way and twoway numerical simulations predict almost the same frequencies. The instability peak is predicted at $450 \mathrm{~Hz}$ which gives a $2.5 \%$ error with comparison to the numerical data. Unexpectedly, the strongest peak predicted by the liner velocity calculations appears at a frequency of $325 \mathrm{~Hz}$, for both: one-way and two-way interaction analysis. This peak is hardly visible in the experimental results. However, the modal analysis (presented in Chapter 5) shows in the vicinity of this frequency a strong first mode at $310 \mathrm{~Hz}$. Knowing that the velocity signal is recorded in the middle of the flexible section, it is most likely that the observed peak at $325 \mathrm{~Hz}$ comes from this mode. Since the pressure and velocity signal are evaluated at different locations this peak is not visible in the pressure spectrum. It shows only that the influence of the vibrating wall one the pressure spectrum is minor.

Generally, the results of the one-way and two-way interaction are in good agreement with the experimental data, for both pressure and velocity signal. Since the thermo-acoustic instabilities are driven by combined fluctuations of the combustion and acoustic field and 
these fields are not changed significantly by the vibrating wall, the pressure data obtained from both numerical simulations is similar. More significant changes are observed in case of the velocity computations. However, here the effect of the vibrating walls is only local and does not have major impact on the overall instabilities in the system. Nevertheless these differences the errors in the predicted peaks do not exceed $6 \%$.

In the work of (Huls, 2006) and (Khatir, Pozarlik, Cooper, Watterson, \& Kok, 2006) a oneway coupling between LES and FEA is performed. Those results have shown similar discrepancies as these in the present work.

\subsubsection{Fluistcom RESUlts}

Next to the Desire configuration, a much more flexible combustion chamber geometry is investigated. The Fluistcom combustion chamber has lower wall stiffness, therefore the feedback from the wall vibrations to the acoustic pressure and further to heat released by the flame is more significant than in case of the Desire geometry. The detailed analysis of the Desire and Fluistcom experimental data can be found in Chapter 2. Here, the experimental signal is compared only against numerical data.

Similar differences between numerical results and experimental data, as during examination of the Desire configuration, are observed for the amplitude of the pressure signal in case of the Fluistcom geometry. The numerical results based on the RMS value show about $40 \%$ under-prediction of the pressure field inside the combustion chamber, as shown in Figure 4-14. In the frequency domain, experimental data presents, next to the forcing peak at 300 $\mathrm{Hz}$, primary thermo-acoustic instabilities at $431 \mathrm{~Hz}$ and $458 \mathrm{~Hz}$ and secondary at $614 \mathrm{~Hz}$. The same peaks are observed in the numerical spectrum. Both computations predict the primary instability at $450 \mathrm{~Hz}$, which gives $4 \%$ or $2 \%$ error, depending on which frequency of instabilities (e.g. $431 \mathrm{~Hz}$ or $458 \mathrm{~Hz}$ ) will be promoted with time during the experiment. Also the valley free of instabilities between $500 \mathrm{~Hz}$ and $600 \mathrm{~Hz}$ is well visible in the numerical models. The secondary instability peak depends on the investigated numerical model and is located at $625 \mathrm{~Hz}$ for the one-way interaction and $638 \mathrm{~Hz}$ for the two-way interaction, which gives $2 \%$ and $4 \%$ discrepancy from experimental results, respectively. Also in the case of Fluistcom calculations, the error between experiment and numerical computations grows with increasing frequency. Up to the frequency of $1000 \mathrm{~Hz}$, the maximum observed error is equal to $6 \%$. The peaks visible in the frequency spectrum of the numerical models, for frequency range $209 \mathrm{~Hz}-263 \mathrm{~Hz}$ are in the vicinity of the first acoustic mode of the combustion chamber, which is located at $220 \mathrm{~Hz}$. Those peaks are visible in the modal analysis of coupled fields (see Chapter 5) as acoustically dominated 
modes and therefore they are also visible in the numerical pressure spectrum of the combustion chamber. No significant differences are observed between the two-way and one-way interaction model. The only visible difference is at the points of the instabilities, where the two-way interaction model predicts the ratio between thermo-acoustic instability and forcing peaks better.
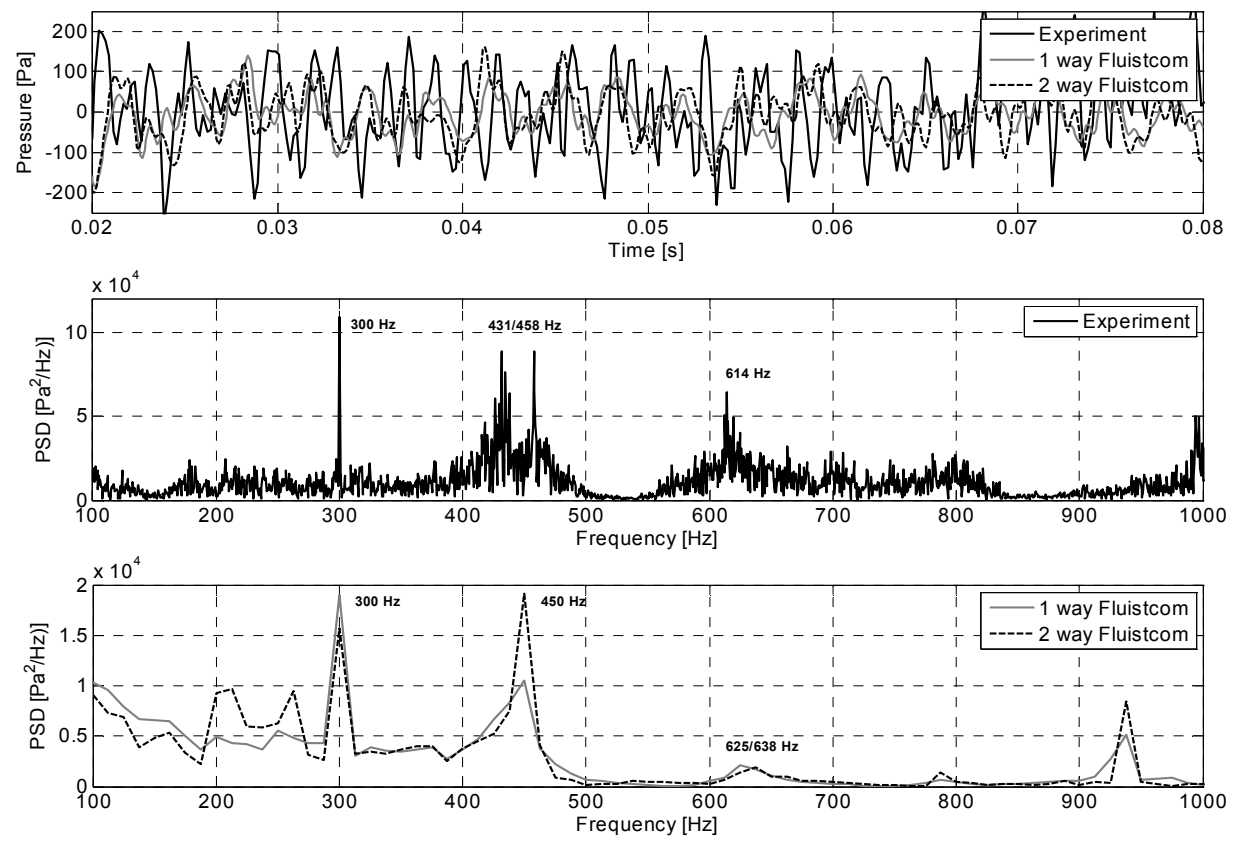

Figure 4-14: Comparison of the experimental and numerical results of the pressure signal in time and frequency domain for the Fluistcom configuration

In case of velocity predictions, the one-way interaction model overestimated the liner velocity magnitude about $92 \%$, as presented in Figure 4-14. This behaviour is in opposition to the pattern of pressure fluctuations which was under-predicted. However, since there is no physical damping involved in the one-way interaction, the higher velocity fluctuations than in reality can be induced by the numerical scheme. For the two-way interaction, where the pressure field is under-predicted with comparison to the experimental data, the RMS velocity results are also under-predicted. The difference with the experimental data is equal to $13 \%$. It means that the geometry changes induced some damping in the system. 

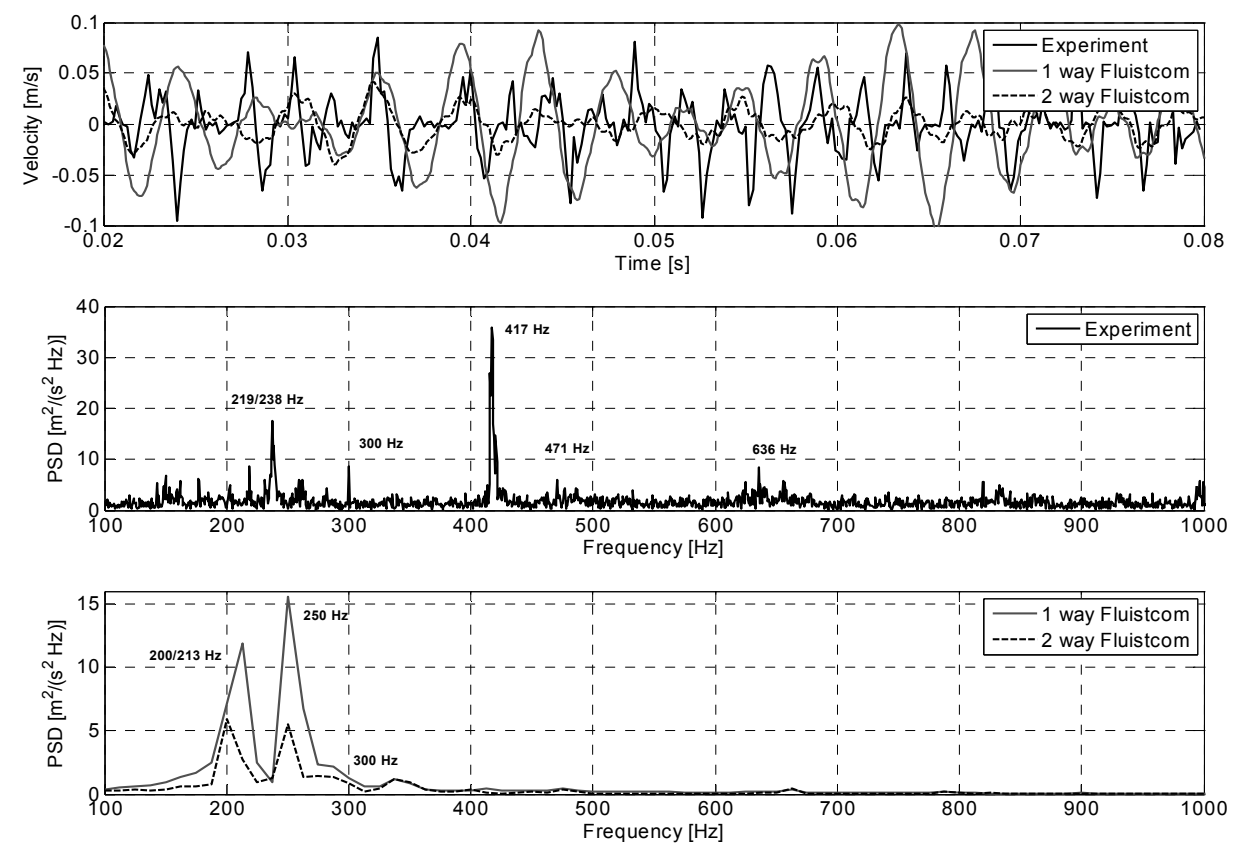

Figure 4-15: Comparison of the experimental and numerical results of the velocity signal in time and frequency domain for the Fluistcom configuration

In the frequency spectrum, the dominant experimental vibration frequency is $417 \mathrm{~Hz}$, followed by $238 \mathrm{~Hz}$ and forcing frequency at $300 \mathrm{~Hz}$. Primary and secondary pressure instabilities are located at locations $431 \mathrm{~Hz}-458 \mathrm{~Hz}$ and $614 \mathrm{~Hz}$ are almost invisible in the velocity spectrum, except small peaks at $471 \mathrm{~Hz}$ and $636 \mathrm{~Hz}$, respectively. The numerical schemes hardly captured the main instability at $417 \mathrm{~Hz}$. This region has to be zoomed to see a small peak. However, at different positions, e.g. in the lower quarter of the liner, the instability peak at $413 \mathrm{~Hz}$ is more visible, see Figure 4-16. This suggests that the numerical liner vibrates with a different mode than the experimental one.

The main vibration frequencies predicted by the numerical codes are located in the range $200 \mathrm{~Hz}-275 \mathrm{~Hz}$. In this region also experimental results show a few significant peaks, see Figure 4-15. The main experimental instability peak was observed at $417 \mathrm{~Hz}$, which is not the case in the numerical prediction. The numerical computations show main peaks at 200 $\mathrm{Hz}, 213 \mathrm{~Hz}$ and $250 \mathrm{~Hz}$. However, the experimental results obtained from the same operation point as the one presented in Figure 4-14 and Figure 4-15, show that depending on some circumstances different vibration frequencies can be induced, see Figure 4-17. In 
this case the main instability frequency is measured at $236 \mathrm{~Hz}$ which is in the neighbourhood of the numerical results, which placed it at $250 \mathrm{~Hz}$.

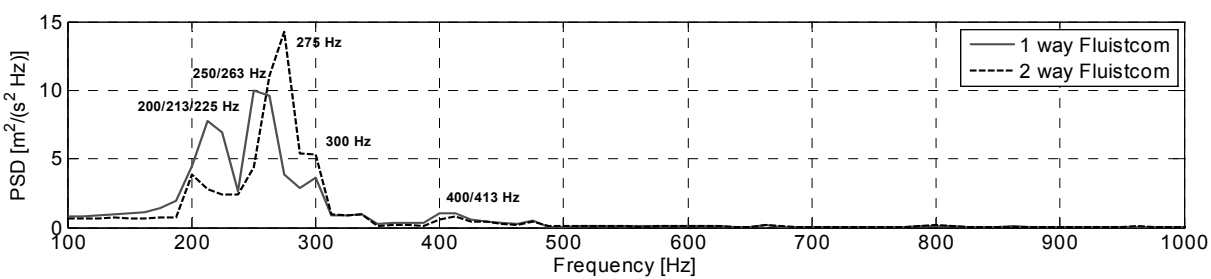

Figure 4-16: Power spectrum density of velocity predicted by the numerical model at location $78 \mathrm{~mm}$ from the bottom edge of the liner
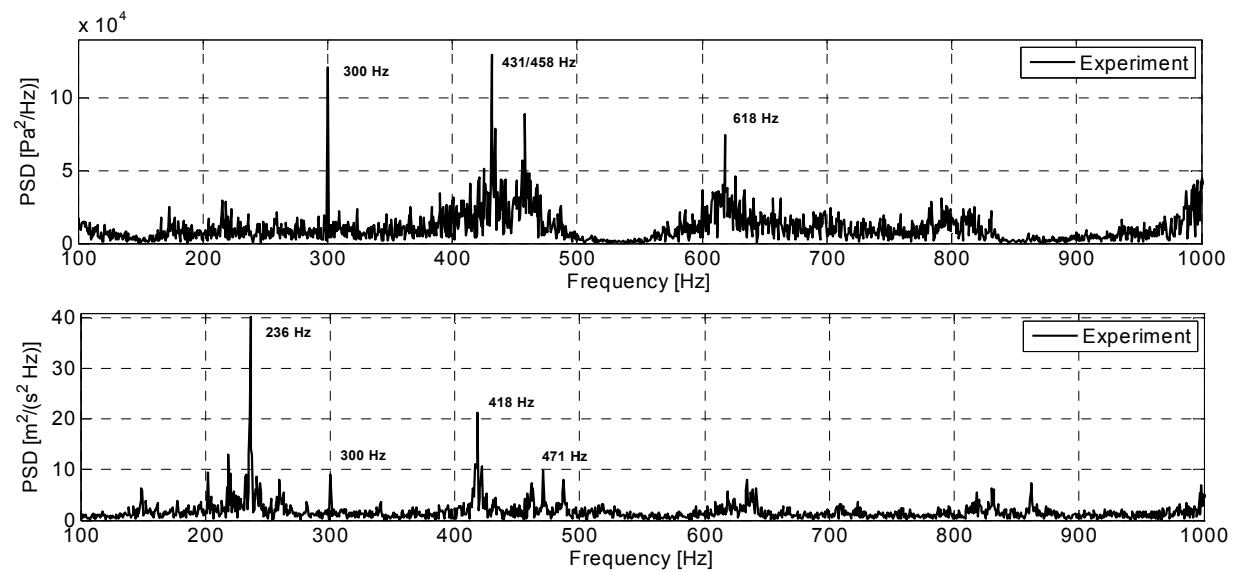

Figure 4-17: Power spectrum density of pressure (top) and velocity (bottom) field obtained during the other experiment

\subsection{Conclusions}

Numerical investigation of the fluid-structure interaction between the combusting flow inside the combustion chamber, acoustics induced by the flame fluctuations and liner walls vibrations has been performed. Two different fluid-structure interaction schemes and two different geometries were employed to predict pressure fluctuations and liner vibrations. The results of the CFD analysis were successfully linked with the FEM code. 
Numerical results have revealed an under-prediction of the amplitude of the pressure fluctuations compared to the results obtained during experiment. The inaccuracy is attributed to damping of the acoustic waves in the CFD code and to a computed physical time interval that was too short. In the aftermath of this error, also smaller mechanical loads are transferred to the CSD code and as result smaller liner velocities are predicted. In all investigated cases, no physical damping was involved in the calculation of the structural wall. Therefore in case of calculation of the Fluistcom geometry, where the wall stiffness was reduced significantly, the one-way interaction presents low frequency modulation with over-prediction of the vibrations amplitude.

Even though the amplitude of the signal predicted by the numerical codes was generally under-predicted, the hazardous frequencies were marked correctly. This suggests a linear behaviour of the system. Next to the forcing peak at $300 \mathrm{~Hz}$, all thermo-acoustic instabilities located in the frequency range $430 \mathrm{~Hz}-450 \mathrm{~Hz}$ were visible on the pressure spectrum. Also the secondary instabilities at around $610 \mathrm{~Hz}-640 \mathrm{~Hz}$ are present in the numerical results. Similar behaviour was observed for the velocity signal. All the major frequencies of instabilities were recognized well by the numerical schemes. For the Fluistcom configuration the main instabilities recognized by the numerical codes were located around $220 \mathrm{~Hz}-250 \mathrm{~Hz}$, whereas the experimental results predicted a higher peak at $417 \mathrm{~Hz}$. However, further experiments showed that under some circumstances the main velocity peak can be shifted to the frequency of $236 \mathrm{~Hz}$. This is in good agreement with the numerical predictions. Apparently the modes of $236 \mathrm{~Hz}$ and $417 \mathrm{~Hz}$ are in competition and the system can switch between these two modes.

In the Desire configuration minor differences between the one-way and two-way interaction model were observed. More differences show the Fluistcom model where the one-way interaction over-predicted the velocity amplitude while the pressure signal was smaller than the experimental one. It is not clear how this discrepancy can be explained. The positions of the instability peaks predicted by both schemes were alike. The two-way interaction model, however presents a higher instability influence on the system. 


\section{ACOUSto-elastic Analysis}

\subsection{INTRODUCTION}

This chapter is devoted to the acousto-elastic analysis. In this analysis mutual interaction between acoustic wave propagation inside the combustion chamber and structural vibrations is investigated. During the computations pressure fluctuations in the combustion chamber are calculated first with the use of the CFD code. Then the CFD results are exported to the FEM code where mutual interaction between acoustic waves and wall vibration is investigated. To reduce the influence of numerical dispersion and dissipation of acoustic waves in the CFD code, only the pressure recorded near the flame region is transferred for the Finite Element Analysis (FEA). Furthermore, the acoustoelastic model is extended and the influence of the cooling passage on the system is taken into account. To simulate acoustic waves next to the vibrating liner, the investigated model is equipped with acoustic elements designed to recognize a structure on one side and a fluid on the other side of the element. Next to the acousto-elastic computation, a modal analysis to mark the hazardous structural, acoustic and coupled modes and eigenfrequencies is performed. Results of the computations using the FEM code are compared with the experimental data.

\subsection{BACKGROUND}

In the previous chapters it was shown, that acoustic waves which travel through the combustion chamber are partially damped by numerical dissipation during the computation process. To avoid restrictions because of the mesh resolution and time step i.e. a fine mesh to simulate the combustion process that would lead to a very small time step in order to resolve the acoustic wave correctly, the acousto-elastic analysis is performed. In this analysis the combustion process is skipped and instead pressure fluctuations caused by the oscillating flame are exported to the FEM code, where further interaction between vibrating 
walls and acoustic pressure inside the combustion chamber is investigated. The acoustoelastic interaction is an analysis of the coupling between acoustic waves and elastic structure deformation, see (Huls, 2006), (Breard, Sayma, Vahdati, \& Imregun, 2002), (Hannink, 2007). This analysis is devoted to an investigation of the mutual interaction between moving walls and the acoustic field, which takes place when a thin, light structure is exposed to the acoustic wave. The interaction is most significant when the acoustic fluid is enclosed by the elastic structure. Then acoustic waves induced in the chamber produce an external pressure loads on the wall surface. Changes in the pressure field induce vibrations of the wall. The vibrating structure is another source of acoustic waves and induces pressure fluctuations in the surrounding fluid, see (Kaczor \& Sygulski, 2005). As a result of interaction between these waves, the acoustic field inside the cavity can be modified as presented e.g. in (Davis, 2008)

The acousto-elastic coupling depends on the properties of the medium through which the acoustic wave travels. For air, only compressibility and inertia effects are taken into account. However, in some cases when the acoustic domain is smaller or of the same order as the viscous layer, the viscous effect should be considered. This exception has also application to the thermal boundary layer, which for gases has a similar thickness as the viscosity layer. When both layers are included in the calculations and when the acoustic domain is small, additional energy dissipation is observed by acoustic wave conversion into heat. This process is examined in detail in (Beltman, 1998) and (Fox \& Whitton, 1980). Knowing that the investigated gas is air, and the Reynolds numbers are high, both viscous and thermal boundary layers have minor influence on the acoustic field in the combustion chamber. Thus the viscous and thermal effects are neglected in the computation process presented here.

\subsubsection{Air InfLuence ON System BeHAViour}

The acousto-elastic model investigated in this chapter considers air at a temperature equal to the temperature of the hot gases inside the combustion chamber. The presence of air trapped by the surrounding structure has its influence. Air introduces additional mass, stiffness and damping, which modifies the performance of a system with vacuum, see (Basten, 2001). The effect of air enclosed by the structure is shortly discussed below:

- Added stiffness - is an effect of compression or rarefaction of air inside the structural domain. It exists for the modes that introduce changes in the total volume, thus it is observed during symmetric modes only, as shown in Figure 5-1. While a symmetric mode is present, the pressure disturbances inside the air cavity 
are in phase with the plate motion. This behaviour results in an increase of the eigenfrequencies of the chamber.

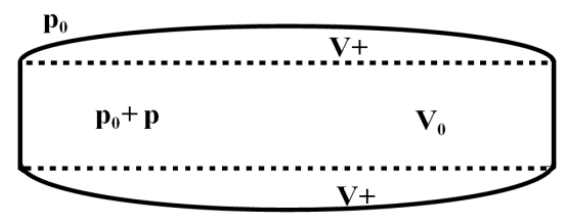

Figure 5-1: Effect of added stiffness

- Added mass - is an effect of air motion. The motion introduces additional inertia forces. The added mass effect is especially important for the asymmetric modes, where changes in the shape pump air from one side to another, as shown in Figure $5-2$. As a result of added mass the eigenfrequencies of the surrounded structure are decrease.

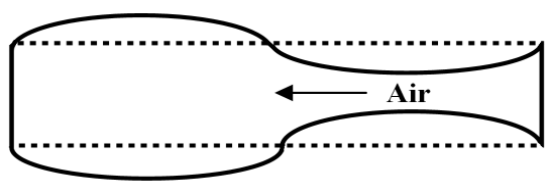

Figure 5-2: Effect of added mass

- Added damping - is an effect of the introduction of a viscous and thermal layer, which decrease the acoustic energy by changing it into heat. Additional damping is also caused by air resistance to the wall movement. Because both, the thermal and viscous layer have are small compared to the enclosure, and the density of air is insignificant compared to the density of the material from which the walls are made of, the added damping does not change the system performances significantly during the present investigations. 


\subsubsection{NEAR AND FAR Field ACOUSTICS}

The acoustic field created by the acoustic source can be divided into two main regions: the near field and the far field acoustics. Figure 5-3 shows the location of both fields in the neighbourhood of the acoustic source. The near field acoustics is located in the vicinity of the acoustic source and strongly depends on the source properties and distance. The far field zone starts at some distance from the source. Beyond the point of transition between the near field and far field acoustics, the wave distribution is mainly independent on the distance from the source.

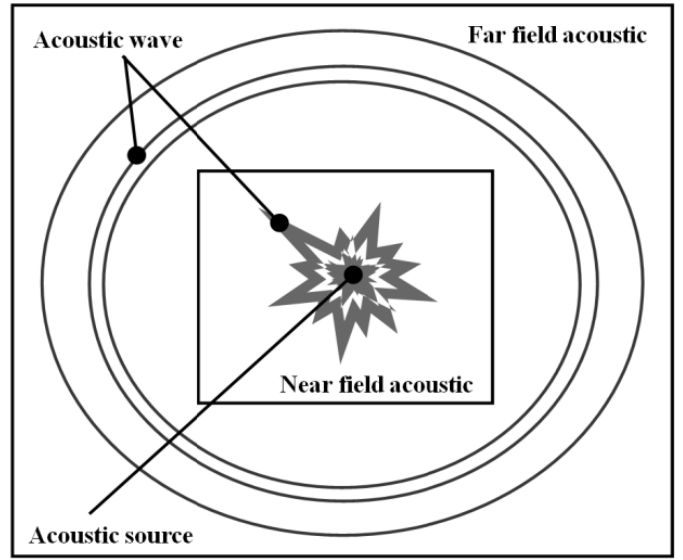

Figure 5-3: Acoustics near and far field

Several definitions of the transition point between near and far field acoustics exist depending on the relation between dimensions of the source, the wave length, diffraction and the type of the acoustic source. An extensive discussion is presented in (Dowling \& Ffowcs Williams, 1983), (Crighton, Dowling, Ffowcs Williams, Heckl, \& Leppington, 1996). As it is pointed out by Dowling and Williams, see (Dowling \& Ffowcs Williams, 1983) a dipole source has a near field but a monopole has not. A monopole sound field decays inversely with distance, whereas a dipole has an intense near field, but most of the pressure fluctuations do not radiate further. The results of the CFD calculation presented in Figure 5-4 and discussed in Chapter 3, show that in the investigated combustion chamber, at some distance from the monopole source, which is the flame, the pressure fluctuations are one-dimensional. Therefore, it is possible to truncate the acoustic domain and export the mean pressure fluctuations from the CFD calculation to the FEA without contamination of 
the investigated signal. This is restricted to the low frequency phenomena as explored in this thesis.

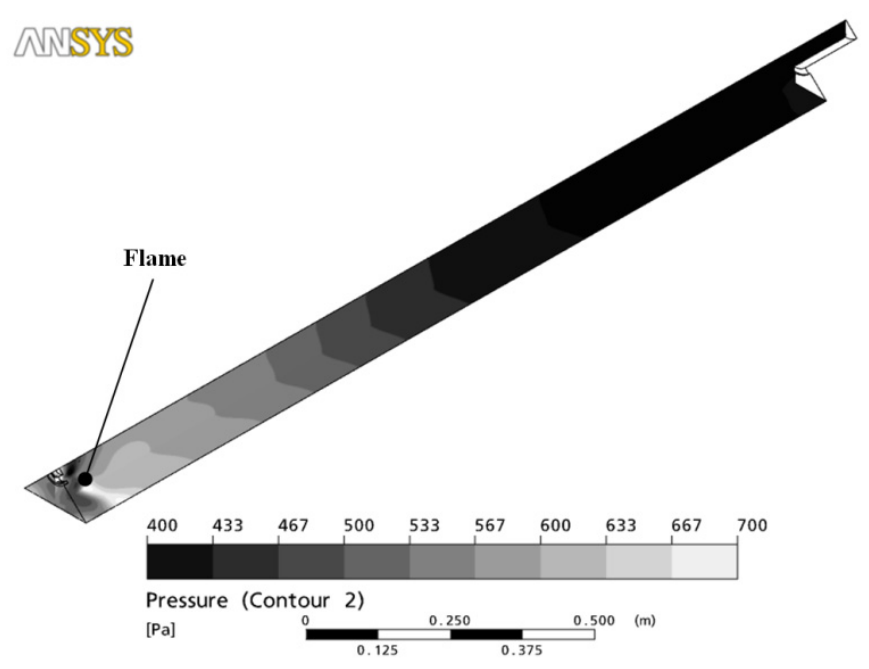

Figure 5-4: A pattern of pressure distribution inside the combustion chamber (from CFD)

\subsubsection{Propagating AND EVANESCENT WAVES}

Depending on the ratio between the two acoustic wave lengths, one produced by the flame and the other produced by the vibrating wall, a different resulting wave is obtained, see (Visser, 2004) and Figure 5-5.
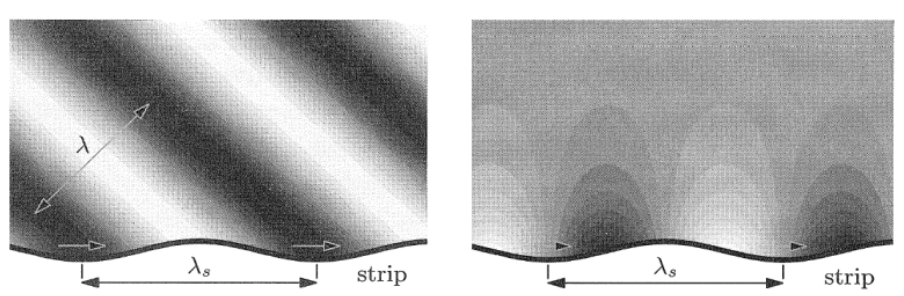

Figure 5-5: Propagating (left) and evanescent wave (right), from (Visser, 2004) 
When the wave length of a transversal wave produced by a flame source $(\lambda)$ is smaller than the length of the wave produced by a vibrating wall $\left(\lambda_{\mathrm{s}}\right)$ a propagation wave is emitted, see Figure 5-5 (left). In contrast, when $\lambda>\lambda_{\mathrm{s}}$ an evanescent wave in the vicinity of the wall is formed, as presented in Figure 5-5 (right). The evanescent type of acoustic wave has only a local character and decays exponentially with the distance from the source.

\subsection{Numerical Model}

As opposed to the calculations presented in Chapter 3 and Chapter 4, not only the combustion chamber and the liner are resolved during the acousto-elastic computations, also the cooling passage can be taken into account. In the cooling passage the flow is not very turbulent and with the absence of a flame, the vibrating walls are the main source of acoustic waves.

For all calculations presented here, the CFD analysis of the combustible flow with time step $1 \mathrm{e}-4 \mathrm{~s}$ is done first in the CFX commercial code (this process is described in detail in Chapter 3). Next, the pressure results from the near flame field are transferred to the FEM code where the wave equation is being solved using acoustic elements designed for this application. This hybrid approach allows combining two separate codes for the investigation. During the acousto-elastic computations, unlike the FSI analysis, where the exchange of information is performed every time step, the data is transferred after the CFD computations are finished. This procedure reduces the computation time, since the timeconsuming data exchange process through the interface connection between the different codes is neglected. Because the truncation is done near the flame front, it is assumed that the transferred acoustic waves are not contaminated by numerical errors and the numerical dissipation and dispersion have minor influence on the acoustic waves. At the location where the truncation is performed, the acoustic waves present already a one-dimensional pattern. Thus, errors introduced during truncation and data transfer between the codes are reduced to a minimum. In the Ansys Multiphysics code, the acoustic waves created by the (flame induced) source in the combustion chamber cavity surrounded by the flexible walls are simulated. The mutual interaction between acoustic waves travelling inside the fluid with vibration of the liner walls is observed. An effect that the modified acoustic waves exert on the flame is not taken into account due to non-availability of combustion and turbulent models in the FEM code. The data exchange process between Ansys CFX and Ansys Multiphysics code is presented schematically in Figure 5-6. 


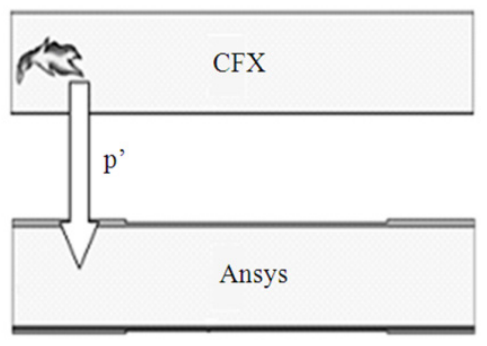

Figure 5-6: Data transfer from CFD to FEM code

The pressure fluctuations taken from the CFX computation are resolved in the Ansys Multiphysics code using the homogenous wave equation, defined as:

$$
\frac{1}{c_{0}^{2}} \frac{\partial^{2} p^{\prime}}{\partial t^{2}}-\frac{\partial^{2} p^{\prime}}{\partial x_{i}^{2}}=0
$$

Equation 5-1 is valid for a medium without mean flow and without temperature gradient. Furthermore, viscous effects are neglected and the medium is assumed to be a continuum. Assuming a harmonic wave of shape $p^{\prime}=p_{o} e^{i \omega t}$, Equation 5-1 simplifies to the Helmholtz equation defined as:

$$
k^{2} p_{0}+\frac{\partial^{2} p_{0}}{\partial x_{i}^{2}}=0
$$

Where: $k$ is a wavenumber, $k=\frac{\omega}{c_{0}}$

Using the Galerkin procedure for discretization and taking into account dissipation of energy due to damping being present at the fluid boundary Equation 5-3 is obtained.

$$
\int_{V} \delta p^{\prime} \frac{1}{c^{2}} \frac{\partial^{2} p^{\prime}}{\partial t^{2}} d V-\int_{V} \delta p^{\prime} \nabla \cdot\left(\nabla p^{\prime}\right) d V+\int_{S} \delta p^{\prime}\left(\frac{r}{\rho_{0} c}\right) \frac{1}{c} \frac{\partial p^{\prime}}{\partial t} d S=0 \quad 5-3
$$


Where: $r$ is the characteristic impedance.

The FEM code employed for the acousto-elastic interaction analysis includes not only acoustic elements for the acoustic pressure waves travelling in the chamber but it is also equipped with solid elements. These elements serve to simulate displacements and stresses inside the walls surrounding the gas cavity, see (Ansys, 2007). The connection between the fluid and structural domain is modelled using additional elements which recognize the structure on one side and fluid on the other side. The interaction between the fluid and structure at the mesh interface includes the acoustic pressure exerting a force on the structure and the structural motion producing an effective fluid load, according to Equation 5-4 and Equation 5-5.

$$
\begin{gathered}
{\left[\boldsymbol{M}_{\boldsymbol{S}}\right]\{\ddot{\boldsymbol{U}}\}+\left[\boldsymbol{C}_{\boldsymbol{S}}\right]\{\dot{\boldsymbol{U}}\}+\left[\boldsymbol{K}_{\boldsymbol{S}}\right]\{\boldsymbol{U}\}=\left\{\boldsymbol{F}_{\boldsymbol{S}}\right\}+[\boldsymbol{R}]\{\boldsymbol{P}\}} \\
{\left[\boldsymbol{M}_{\boldsymbol{F}}\right]\{\ddot{\boldsymbol{P}}\}+\left[\boldsymbol{C}_{\boldsymbol{F}}\right]\{\dot{\boldsymbol{P}}\}+\left[\boldsymbol{K}_{\boldsymbol{F}}\right]\{\boldsymbol{P}\}=\left\{\boldsymbol{F}_{\boldsymbol{F}}\right\}-\rho_{0}[\boldsymbol{R}]^{\boldsymbol{T}}\{\ddot{\boldsymbol{U}}\}}
\end{gathered}
$$

In Equation 5-4 and Equation 5-5, the coupling matrix $\mathbf{R}$ takes into account the direction of the normal vector defined for each pair of coincident fluid and structural element faces. The positive direction of the normal vector is defined to be outward from the fluid mesh and towards to the structure, see (Ansys, 2007). $\mathbf{F}_{\mathbf{S}}$ and $\mathbf{F}_{\mathbf{F}}$ are structural and fluid forces, respectively.

Both the structural and fluid load quantities that are produced at the fluid structure interface are functions of unknown nodal degrees of freedom. The governing finite element matrix equations then become:

$$
\left[\begin{array}{cc}
\boldsymbol{M}_{\boldsymbol{S}} & \mathbf{0} \\
\rho_{0} \boldsymbol{R}^{T} & \boldsymbol{M}_{\boldsymbol{F}}
\end{array}\right]\left[\begin{array}{c}
\ddot{\boldsymbol{U}} \\
\ddot{\boldsymbol{P}}
\end{array}\right]+\left[\begin{array}{cc}
\boldsymbol{C}_{S} & 0 \\
0 & \boldsymbol{C}_{\boldsymbol{F}}
\end{array}\right]\left[\begin{array}{l}
\dot{\boldsymbol{U}} \\
\dot{\boldsymbol{P}}
\end{array}\right]+\left[\begin{array}{cc}
\boldsymbol{K}_{S} & -\boldsymbol{R} \\
0 & \boldsymbol{K}_{F}
\end{array}\right]\left[\begin{array}{l}
\boldsymbol{U} \\
\boldsymbol{P}
\end{array}\right]=\left[\begin{array}{c}
\boldsymbol{F}_{S} \\
\boldsymbol{F}_{F}
\end{array}\right]
$$

As can be seen in the above equation, the nodes on the fluid structure interface are characterized by both the displacement and pressure degrees of freedom. For more details about coupling between fluid and structure loads in the FEM Ansys package see (Ansys, 2007). 


\subsubsection{GEOMETRY AND BOUNDARY CONDITIONS}

The spatially averaged pressure fluctuations in the CFX calculations are taken from the near flame region and implemented to the acousto-elastic model as boundary conditions. The combustion chamber geometry is simplified to a rectangular shape without the burner and exhaust pipe. A boundary absorption coefficient $\beta=\frac{r}{\rho_{0} c}$ is calculated based on changes in the cross-section area and imposed as the outlet boundary conditions. At the inlet, an acoustically hard wall is imposed. Three different types of elements are used. For the structural modelling, SHELL63 elements with the element real constant equal to the thickness of the individual liner sections are employed. The SHELL63 elements are based on the Kirchhoff-Love theory, as presented in (Belov, Kaplunov, \& Nolde, 1999). The transverse shear stresses are not taken into account. Due to the small ratio between the thickness of the liner wall and other wall dimensions (always below 0.1) and due to a bending stress which changes linearly with the body thickness, the Kirchhoff-Love theory is valid for the liner configuration presented in this thesis. The air cavities of the combustion chamber and cooling passage are represented by the FLUID30 acoustic elements. Two types of FLUID30 elements are used. The major part of the acoustic domain is modelled with regular FLUID30 acoustic elements. One layer of the FLUID30 elements, with ability to recognize structure on one side and fluid on the other, is placed next to the wall. The governing acoustic equation is discretized taking into account the coupling between acoustic pressure and structural motion at the interface, see Equation 5-6. Moreover, for the case with cooling passage geometry, in order to prevent direct connection of the pressure nodes from the combustion chamber with nodes from the cooling passage at the structure interface, the model of the liner is divided into two equal parts. These parts are connected together by stiff degrees of freedom and they have exactly the same properties as the original model. Therefore, any change in one of the structural sections forces the same behaviour in the other section. This configuration assures correct transfer of loads between the acoustic fluid and structural elements. The cross-section of the numerical model with pointed different types of elements and connections is presented in Figure 5-7.

The model includes all four liner walls. The walls are clamped on both ends. Material properties of the stainless steel and the temperature field inside the combustion chamber are equal to the average temperature calculated during the CFD analysis, see Chapter 3 . The speed of sound inside the combustion chamber and in cooling passage is taken at the average temperature field, observed during previous research. Physical damping is not included into the computation model. However, the liner vibrations can be affected by air resistance inside the combustion chamber. The effect of added stiffness and mass (similar to the two-way interaction analysis) are taken into account. In case of the model with the 
cooling passage, additional damping can be provided by the cooling flow. A longitudinal cross-section of the numerical model geometry (without cooling passage) together with the boundary conditions is presented in Figure 5-8.

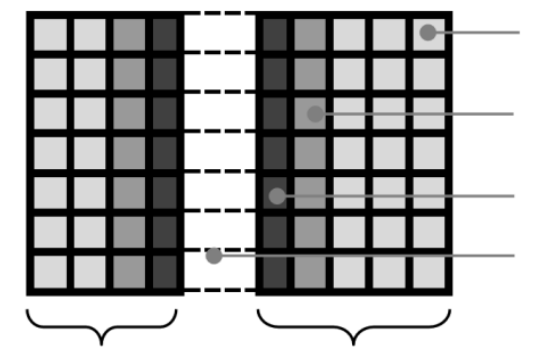

Cooling passage Combustion chamber

Fluid elements (FLUID30)

Fluid elements with ability

to recognize structure

(FLUID30)

Structural elements (SHELL63)

Stiff connection between structural parts

Figure 5-7: Transverse cross-section of the acousto-elastic model

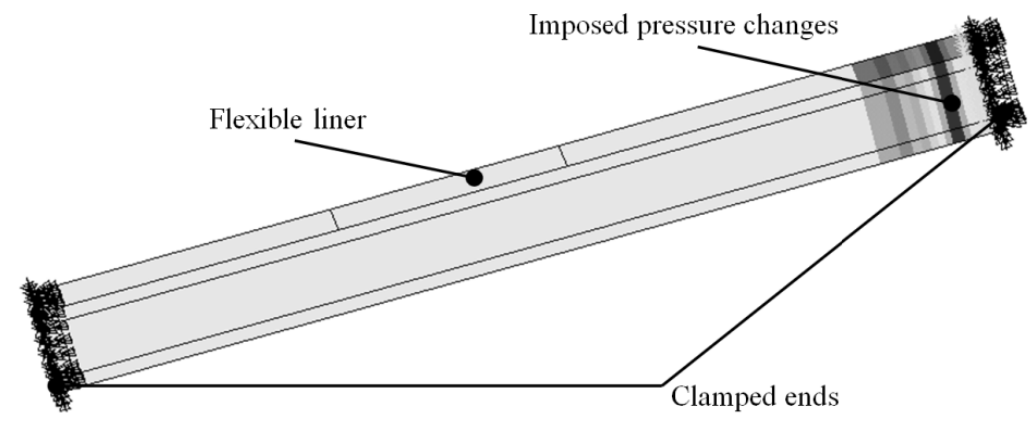

Figure 5-8: Numerical acousto-elastic model with boundary conditions (for better visibility the cooling passage is not included)

Two different liner configurations are investigated here, the Fluistcom configuration with thin and long flexible part and the Desire configuration with a stiff flexible part. For detailed dimensions of both configurations see Chapter 2 . 


\subsection{Modal Analysis}

To determine the vibration characteristics of the combustion chamber walls and the influence of the hot gases trapped inside, a modal analysis is performed. The eigenfrequencies and shapes of the structural, acoustics and coupled modes are compared to each other and a relation between them is observed.

The same numerical model as for the acousto-elastic analysis is employed to investigate the coupled modes. The difference lies only in the boundary conditions used for the model. For the modes investigation there is no need to transfer pressure fluctuations from the CFX code, therefore this data is not included in the modal analysis. Also the impedance at the outlet from the combustion chamber is not taken into account. Computations of the coupled modes include structural and fluid domains together. The structural domain has clamped conditions on the top and bottom edges. For the acoustic modes investigation, the numerical domain is reduced to a fluid part only surrounded by acoustically hard walls. This configuration assures full reflection of acoustic waves without possibility of the liner walls to vibrate.

In the coupled modes analysis, the points where the structural and acoustic eigenfrequencies intersect are called isochronism points. The isochronism points are hazardous for combustion systems because the acoustic and structural eigenfrequencies can be fed simultaneously and they can finally lead to unstable combustion and to liner failure. The uncoupled acoustic eigenfrequencies for the model with rigid walls are defined according to Equation 5-7. The structural resonance frequencies of a plate with clamped degrees of freedom can be calculated using Equation 5-8.

$$
\begin{gathered}
f_{l m n}=\frac{c_{0}}{2} \sqrt{\frac{l_{a}^{2}}{l_{x}^{2}}+\frac{m_{a}^{2}}{l_{y}^{2}}+\frac{n_{a}^{2}}{l_{z}^{2}}} \\
f_{l m}=\frac{\lambda_{l m}^{2}}{2 \pi w_{p}^{2}} \sqrt{\frac{E t_{p}^{2}}{12 \gamma\left(1-v^{2}\right)}}
\end{gathered}
$$

Where: $l_{a}, m_{a}, n_{a}$ is the number of half acoustics waves in $x, y$ and $z$ direction. $w_{p}$ and $t_{p}$ are plate width and thickness, respectively. $\gamma$ is mass of the plate per unit area and $\lambda_{l m}$ is tabularized factor, depending on the type of a support and dimension of the plate. Factor $\lambda_{l m}$ can be found in (Blevins, 1984). 
Validation of the numerical data against the analytical results for a given plate with clamped edges is presented in Appendix A.

For both geometries, i.e. Desire and Fluistcom, the total length of the liner and its cross section area are kept unchanged. Due to the assumption of acoustically hard walls, the acoustic mode shape and natural frequencies stay the same for both models. In case of coupled modal analysis, the influence on the natural modes and frequencies has to be caused by the flexible section located between the stiff liner parts. This section is different with respect to the length and thickness for both models. That is why the structural and coupled eigenfrequencies and modes are different for the Desire and Fluistcom combustion chamber configuration. The frequencies of the isochronism points are similar for both geometries, however, different structural modes are observed in the vicinity of the isochronism point. Thus the effect of the coupled modes on the system behaviour can be different.

\subsection{Numerical Results}

The results of the acousto-elastic and modal analysis are presented here in two sections, one for the Desire combustion chamber configuration and a second for the Fluistcom geometry. Furthermore, these results are divided into acousto-elastic results and modal analysis. In both cases the geometries with combustion chamber only and with combustion chamber and cooling passage are discussed. All numerical data is compared with experimental results.

\subsubsection{Desire - ACOusto-elastic Analysis}

Two different geometries i.e. with and without cooling passage are investigated during the acousto-elastic analysis of the Desire configuration. As can be seen in Figure 5-9, the amplitude of pressure changes during both acousto-elastic computations agrees well with the experimental results. 

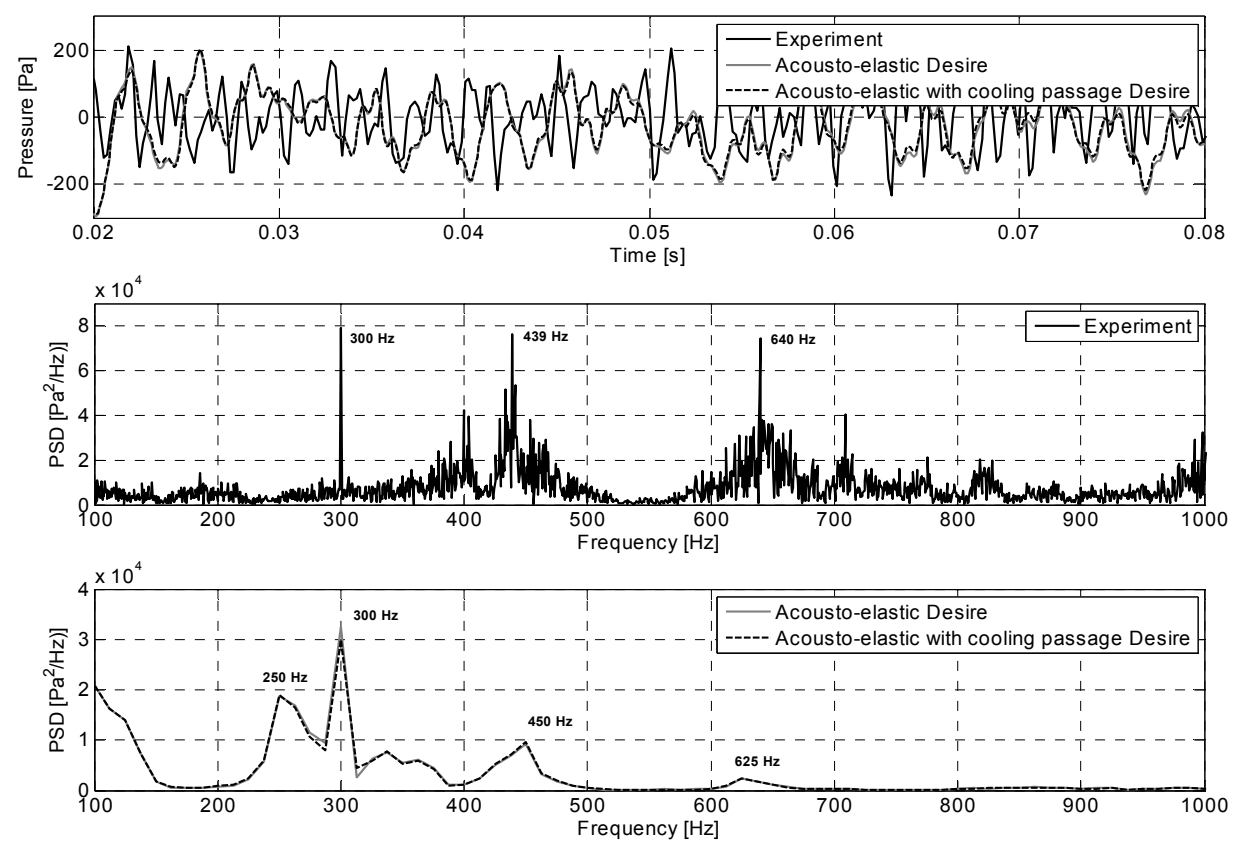

Figure 5-9: Comparison of the numerical pressure data with experimental results for the acoustoelastic analysis

This is an improvement compared to the fluid-structure interaction model, where the pressure amplitude was under-predicted due to numerical dissipation. Also the frequency of main instabilities is predicted correctly. The experimental peaks predicted at $300 \mathrm{~Hz}, 439$ $\mathrm{Hz}$ and $640 \mathrm{~Hz}$ are visible in the numerical spectrum. The forcing frequency of $300 \mathrm{~Hz}$ is the dominant peak. Primary instabilities are predicted at $450 \mathrm{~Hz}$ and a secondary at $625 \mathrm{~Hz}$, which gives in both investigated cases a percentage error below 3\%. The instabilities predicted by the numerical scheme at $250 \mathrm{~Hz}$ are not visible in the experimental spectrum due to feedback from the acoustic field to the flame. Since the flame is not modelled this feedback is not included in the model. However, as can be seen in the work of (Van Kampen, 2006) the thermo-acoustic instabilities under some circumstances can occur during experiment at two possible frequencies, $441 \mathrm{~Hz}$ and $239 \mathrm{~Hz}$. The latter frequency is pointed by the acousto-elastic model at $250 \mathrm{~Hz}$. Similar to the fluid-structure interaction analysis (see Chapter 4) also in the acousto-elastic analysis the forcing peak dominates the thermo-acoustic instabilities. Improvement of the pressure signal resolved by the CFD code, as well as introduction of the pressure source which depends on the incoming wave should improve obtained results. 
In the velocity field, both the experimental and numerical data show similar fluctuation amplitudes, see Figure 5-10. The forcing frequency at $300 \mathrm{~Hz}$ and instability frequency at $439 \mathrm{~Hz}$ are present in the numerical spectrum. The forcing frequency is exactly at $300 \mathrm{~Hz}$ and the vibrations coming from the thermo-acoustic instabilities are placed at $425 \mathrm{~Hz}$. This gives an error less than $3 \%$ in case of the prediction of instabilities Similar to the pressure results, also here the dominant peak comes mainly from the forcing frequency. The instability presented in the numerical pressure spectrum at $250 \mathrm{~Hz}$ is hardly visible in the velocity spectrum. However, at this frequency there is no coupling between acoustic mode and structural mode, see Table 5-2, thus the effect of pressure changes on the vibration pattern is insignificant.
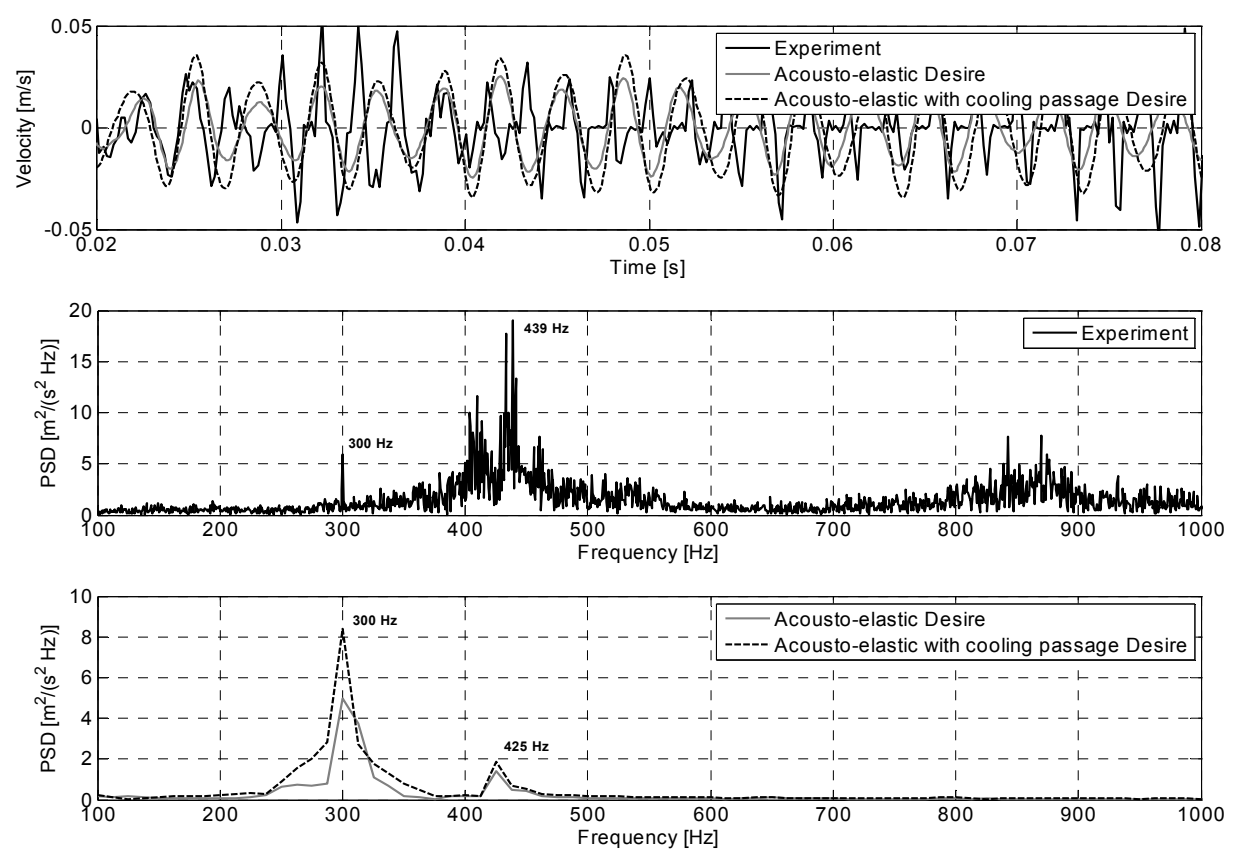

Figure 5-10: Comparison of the numerical velocity with the velocity obtained during the experiment

Only minor differences between results for model with and without cooling passage are observed, see Figure 5-9. Both models predicted the same amplitude of pressure fluctuations and frequency of the instabilities (it is difficult to distinguish the numerical profiles since they are on top of each other). More significant differences are visible in case of the velocity results, where the additional mass of air induces more significant vibrations of the liner. However, these changes are not translated to a frequency shift. Also they do not 
induce additional pressure changes in the acoustic and velocity fields. Thus both models predicted the same instability frequency at $425 \mathrm{~Hz}$.

The advantage of the acousto-elastic analysis is the opportunity to simulate pressure oscillations accurately inside the cooling passage which is difficult to achieve using the fluid-structure interaction model described in Chapter 4. Since the flame is not present in the cooling channel and the flow of the coolant is not very turbulent, the acoustic waves present in the cooling channel are created mainly by the vibrating walls and sound transmission by connected channels. In Figure 5-11, experimental and numerical data of pressure oscillations at the location of pressure transducer P5 (see Chapter 2) is presented. This pressure transducer is one of the two placed in the cooling passage. Because the transducer is located close to the vibrating wall and far from the cooling air inlet it was chosen for pressure data comparison. The amplitude of the pressure fluctuations predicted by the numerical code is slightly bigger than the experimental. In the experimental setup the acoustic wave is damped more than in the numerical model. Here pre-stress and additional stiffness from the welded parts have influence. However, as mentioned earlier, a damping coefficient is not included in the material properties of the liner walls.

The main frequencies observed during experiment and computations in the cooling passage cavity are related more to the pressure changes inside the combustion chamber than to the frequencies of the vibrating flexible walls. This behaviour indicates that a significant part of the acoustic waves can travel to the cooling passage by the bypass connection between cooling passage and cooling chamber. Examination of the vibration of the stiff liner part at the location where pressure transducer P5 is placed shows that the vibration pattern here is different from the flexible section, see Figure 5-12. Moreover, this vibration pattern resembles more the acoustic wave recorded in the cooling passage. Most likely, the acoustic waves created inside the cooling passage are a product of the entire structure which vibrates, and not only of the flexible part. Additionally, connections of pressure transducers, thermocouples and the construction of the bypass between cooling passage and cooling chamber induce additional acoustic sound. 

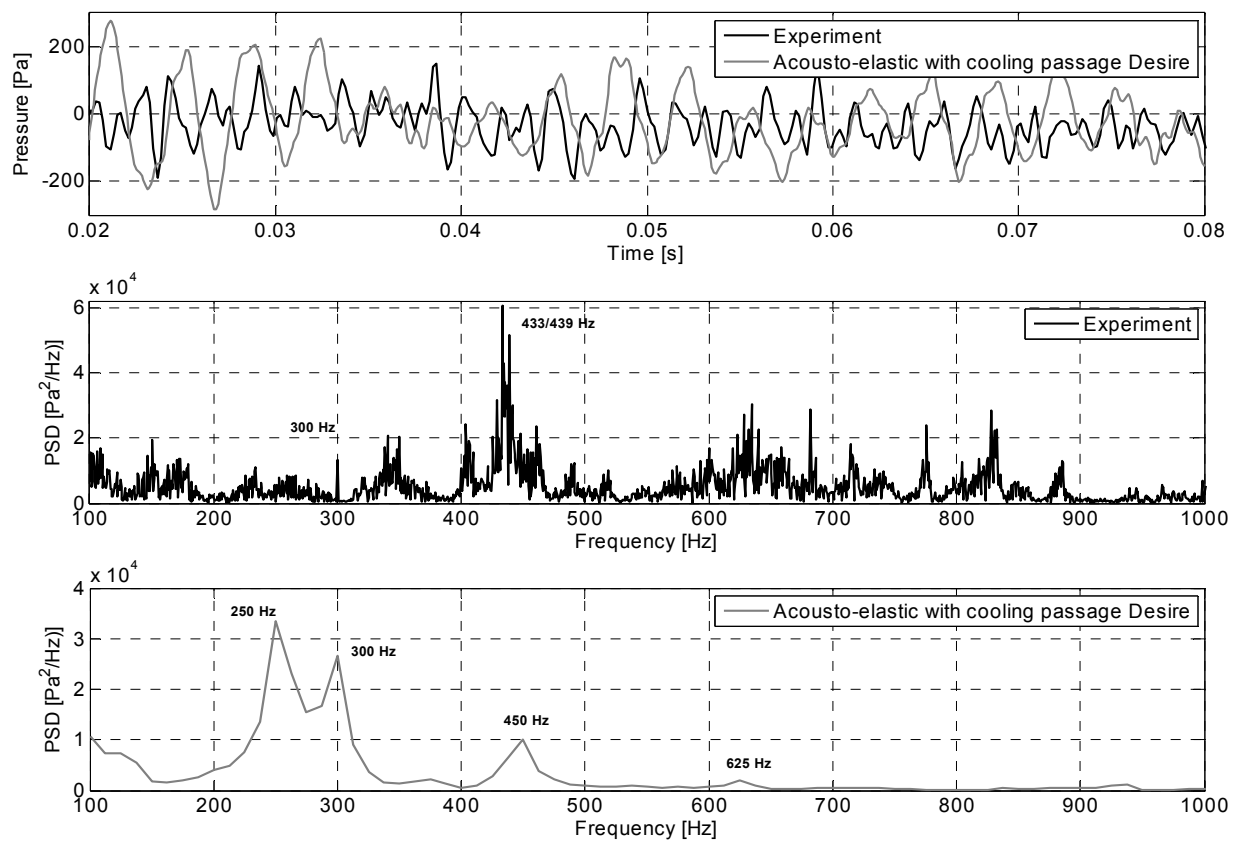

Figure 5-11: Experimental and numerical pressure inside the cooling passage, at location of pressure transducer P5

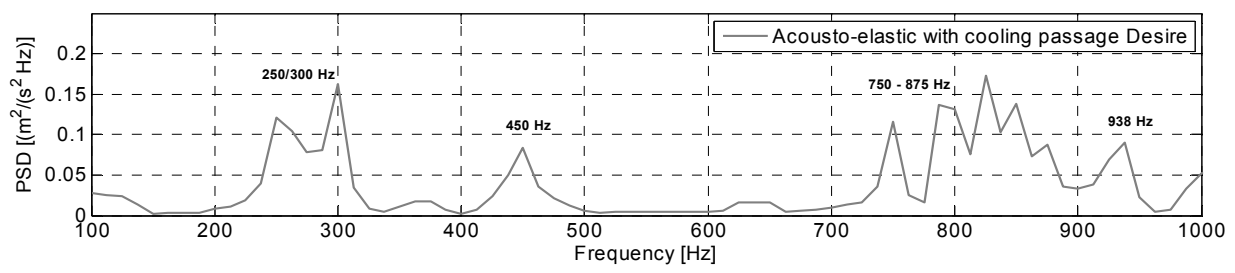

Figure 5-12: Velocity spectrum at position of pressure transducer P5

\subsubsection{FluISTCOM - ACOUSTO-ELASTIC ANALYSIS}

In this section the Fluistcom liner configuration is investigated. The more flexible liner section should be able to change the pressure distribution over the liner. However, as is shown in Figure 5-9 and Figure 5-13 only minor pressure differences, between the Desire and Fluistcom configuration, due to the vibrating liner, are observed in the combustion chamber during numerical computations. This behaviour suggests that 
vibrating walls have only a local effect on the pressure field and this effect is not translated to the pressure changes in the upstream part of the combustion chamber. Thus, interaction between the acoustic wave induced by the flame and the one coming from the vibrating walls resembles an evanescent wave. Also experimental results of both liner configurations are very similar. Therefore, the acousto-elastic analysis of the Fluistcom configuration presents similar errors as the acousto-elastic analysis of the Desire liner geometry presented in the previous section. This means that the forcing peak is exactly at the position of the experimental one, and the primary and secondary instabilities are predicted with small error, with respect to the experimental results.
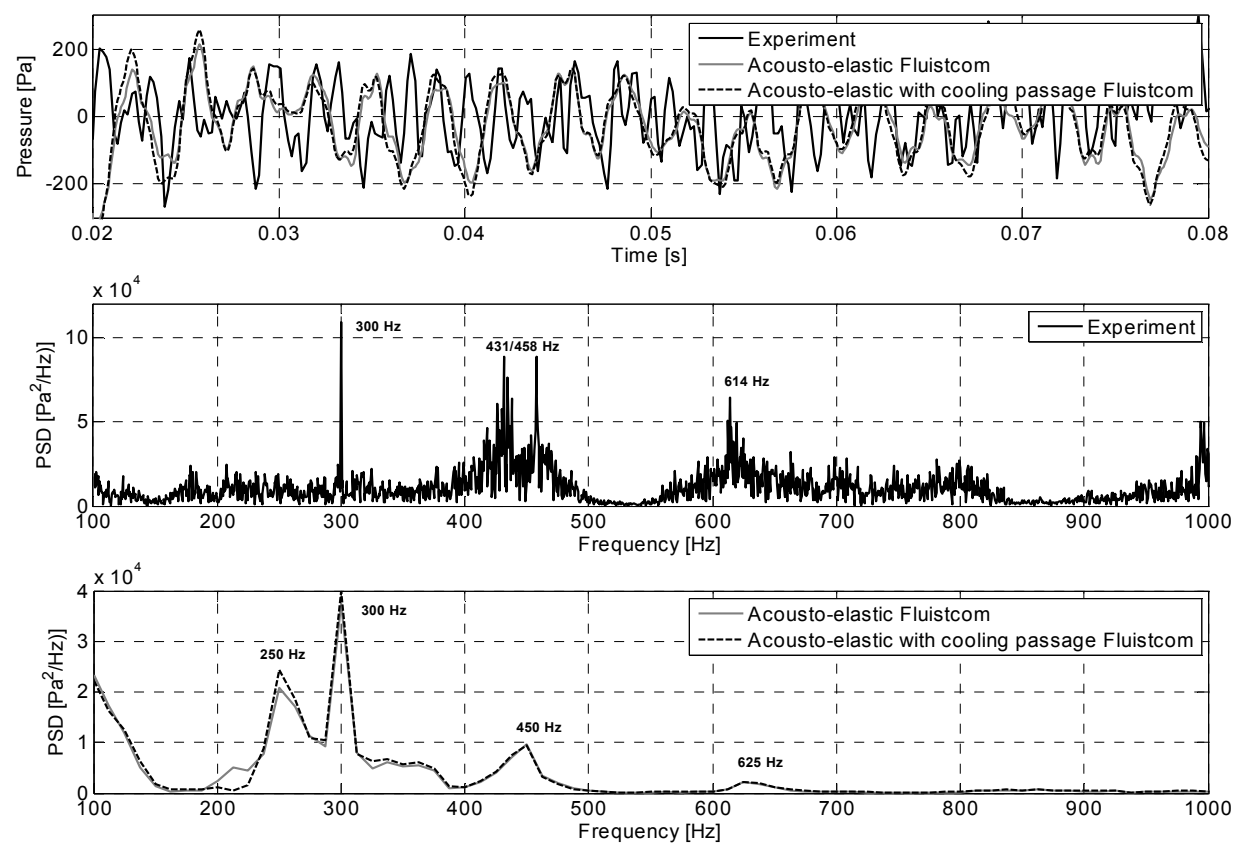

Figure 5-13: Comparison of the numerical pressure data with experimental results for Fluistcom liner configuration

The wall velocity amplitude predicted by the acousto-elastic models with and without cooling passage is under-predicted with respect to the experimental results (based on RMS values), see Figure 5-14. Nevertheless, the acousto-elastic model including the cooling passage places the main vibration frequency at $438 \mathrm{~Hz}$. This is in the vicinity of the major experimental velocity peak located at $417 \mathrm{~Hz}$. The acousto-elastic model without cooling passage missed this frequency of vibrations entirely. Thus, the vibrations at $438 \mathrm{~Hz}$ are 
induced by local pressure changes inside the combustion chamber in the neighbourhood of the vibrating wall. The remaining frequencies of vibrations in both models are predicted similarly. The forcing frequency is located at $300 \mathrm{~Hz}$ which is also exactly the case of the experimental setup. Furthermore, in the range $200 \mathrm{~Hz}-300 \mathrm{~Hz}$, several velocity peaks are predicted at frequencies $213 \mathrm{~Hz}, 225 \mathrm{~Hz}, 250 \mathrm{~Hz}$ and $275 \mathrm{~Hz}$. During the experiment the observed vibrations in this frequency range are pointed at $219 \mathrm{~Hz}, 238 \mathrm{~Hz}$ and $260 \mathrm{~Hz}$, which is in good agreement with the numerical data.
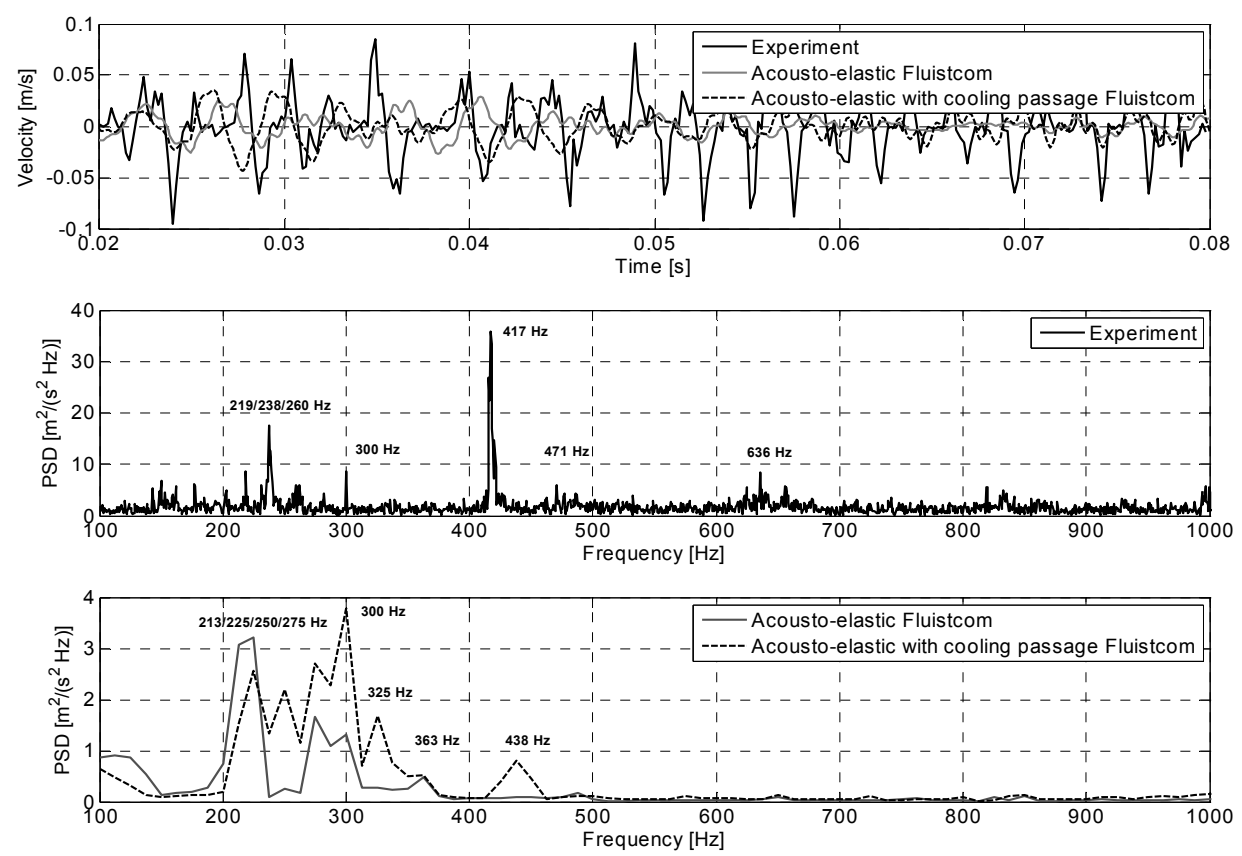

Figure 5-14: Comparison of the numerical velocity data with experimental results for Fluistcom liner configuration

In the cooling passage the main pressure oscillations are predicted well by the acoustoelastic model, see Figure 5-15. The instabilities at $239 \mathrm{~Hz}$ and $431 \mathrm{~Hz}$ are predicted by the model at $250 \mathrm{~Hz}$ and $450 \mathrm{~Hz}$, respectively. Similar to the Desire configuration, also here the acoustic waves originating from the cooling passage are induced by wall vibration, see Figure 5-16. However, the participation of the flexible part in this process is more significant. 

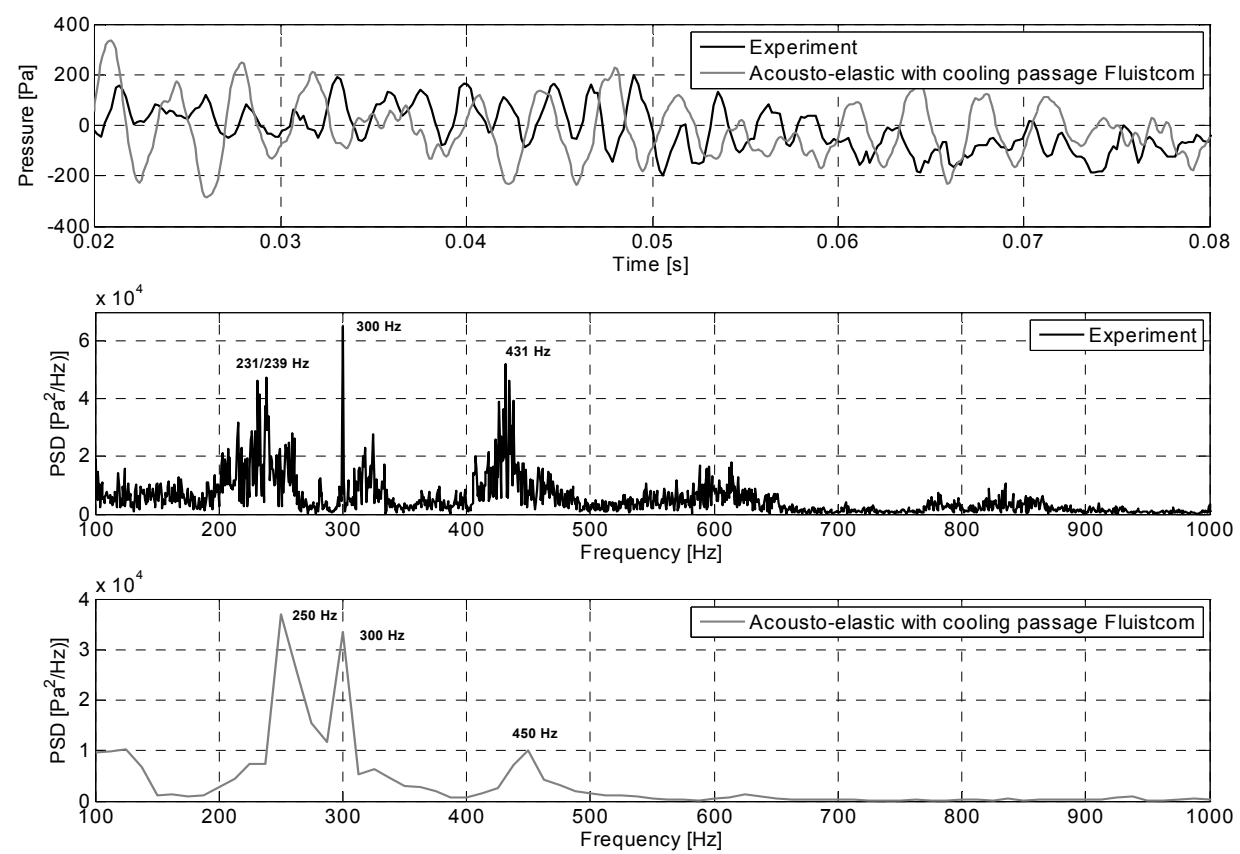

Figure 5-15: Pressure comparison in the cooling passage (P5)

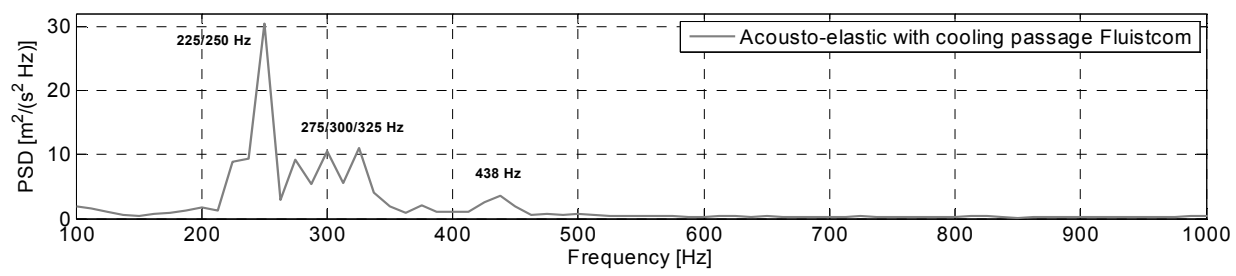

Figure 5-16: Liner velocity spectrum at location of pressure transducer P5

\subsubsection{MOdAl ANALYSIS OF DESIRE}

Next to the acousto-elastic analysis, the computation of acoustic and coupled modes is performed. Due to the assumption that the wall thickness has no influence on the pressure field inside the combustion chamber, the acoustic modes are the same for the Desire and Fluistcom liner configuration. Furthermore, since the combustion chamber geometry is 
characterized by high a ratio between length and width/depth of the walls, acoustic modes in the investigated frequency range up to $1000 \mathrm{~Hz}$ present only longitudinal shape.

Four acoustic modes are distinguished in the investigated frequency range. They are presented in Table 5-1 and Figure 5-17. The modes show a different pressure pattern over the combustion chamber. The first acoustic mode at $222 \mathrm{~Hz}$ has a uniform pressure distribution over the length. This mode represents a half of the acoustic wave in longitudinal direction. The other acoustic modes show a pattern of one acoustic wave, one and a half acoustic wave and two acoustic waves, respectively, see Figure 5-17. Depending on the number of half waves in the longitudinal direction, the acoustic modes are numbered as $1 \mathrm{a}, 2 \mathrm{a}$ etc, and structural modes as $1 \mathrm{~s}, 2 \mathrm{~s}$ etc. For the geometry configuration presented in this thesis, the acoustic modes presenting local air compression or rarefaction (modes $2 \mathrm{a}$, $3 \mathrm{a}$ etc.) are hazardous. These modes are particularly dangerous, because local significant pressure changes are able to induce strong liner vibrations.

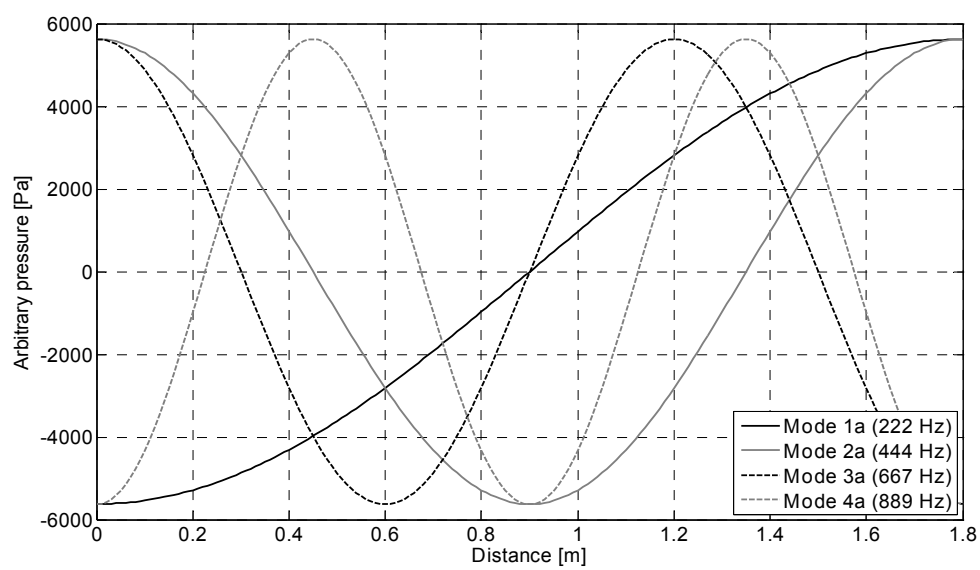

Figure 5-17: Acoustic modes (pressure distribution along centreline)
Mode number
1
2
3
4

$\begin{array}{lllll}\text { Eigenfrequency [Hz] } & 222 & 444 & 667 & 889\end{array}$

Table 5-1: Acoustic eigenfrequencies of the combustion chamber

The influence of the acoustic modes on the liner structure and vice versa is taken into consideration in the coupled modes analysis. During the coupled modes computations the structural domain is coupled with the fluid cavities according to the scheme explained in 
the earlier part of this chapter. Since the number of modes in the frequency range up to $1000 \mathrm{~Hz}$ can be significant, the analysis is restricted to the first fifty coupled modes. During the coupling modal analysis, due to the possible interaction between acoustic and structural modes, mainly the modes in the vicinity of the isochronism frequencies are examined. Furthermore, modes which present global longitudinal pressure changes are taken into consideration. The pressure distribution inside the combustion chamber and the liner displacement pattern of the chosen modes are presented in Figure 5-18 and Figure 5-19, respectively. Furthermore, in Table 5-2 the natural frequencies of the modes are listed.

The most energetic modes giving the strongest feedback to the system are the coupled modes, which present significant air compression or rarefaction in the vicinity of the flexible section. These volumetric or synchronized modes are characterized by major volume changes. In the investigated combustion chamber configuration the synchronized modes appear at frequencies of $429 \mathrm{~Hz}, 446 \mathrm{~Hz}, 657 \mathrm{~Hz}$ and $670 \mathrm{~Hz}$. Two first modes located at $429 \mathrm{~Hz}$ and $446 \mathrm{~Hz}$ present a symmetrical pressure distribution with one local air rarefaction or compression (thus pattern of 2 a symmetrical mode). Two other located at 657 $\mathrm{Hz}$ and $670 \mathrm{~Hz}$ are unsymmetrical 3a modes, see Figure 5-18. These four modes are coupled only with symmetrical structural modes (3s and 5s), as presented in Figure 5-19. These modes can lead to high amplitude oscillations when excited. They are indicated by underlining them in Table 5-2.

\begin{tabular}{ccccccccc} 
Number & 1 & 2 & 3 & 4 & 5 & 6 & 7 & 8 \\
\hline Eigenfrequency [Hz] & 219 & 312 & 354 & $\underline{\mathbf{4 2 9}}$ & $\underline{\mathbf{4 4 6}}$ & 542 & $\underline{\mathbf{6 5 7}}$ & $\underline{\mathbf{6 7 0}}$
\end{tabular}

Table 5-2: Eigenfrequencies of selected coupled modes

At a frequency equal to $219 \mathrm{~Hz}$ another isochronism point exists. The structural mode at that frequency is coupled with an acoustic mode. As it was explained above, this acoustic mode due to the uniform pressure distribution gives only minor feedback to the system.

The other modes at $312 \mathrm{~Hz}, 354 \mathrm{~Hz}$ and $542 \mathrm{~Hz}$ are modes dominated by the structure but they present a similar pressure distribution as the acoustic modes. However, because there is no acoustic mode in the vicinity, these modes present only a weak coupling between structural and fluid domain. As a consequence, they do not have a significant influence on the thermo-acoustic instabilities. 

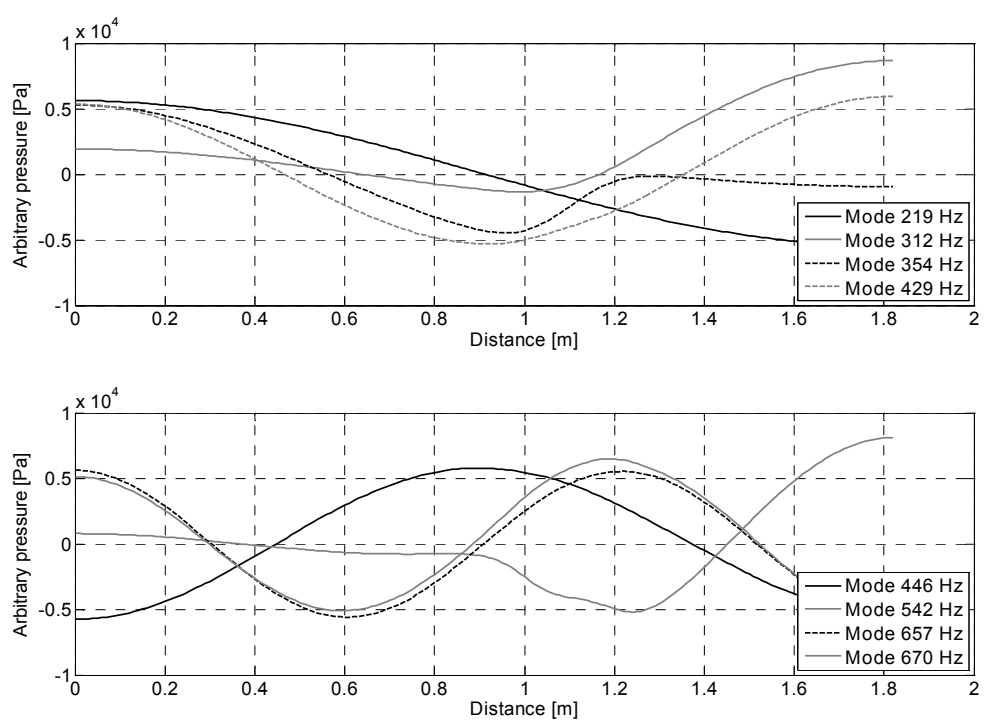

Figure 5-18: Selected coupled modes (pressure distribution pattern along centreline)

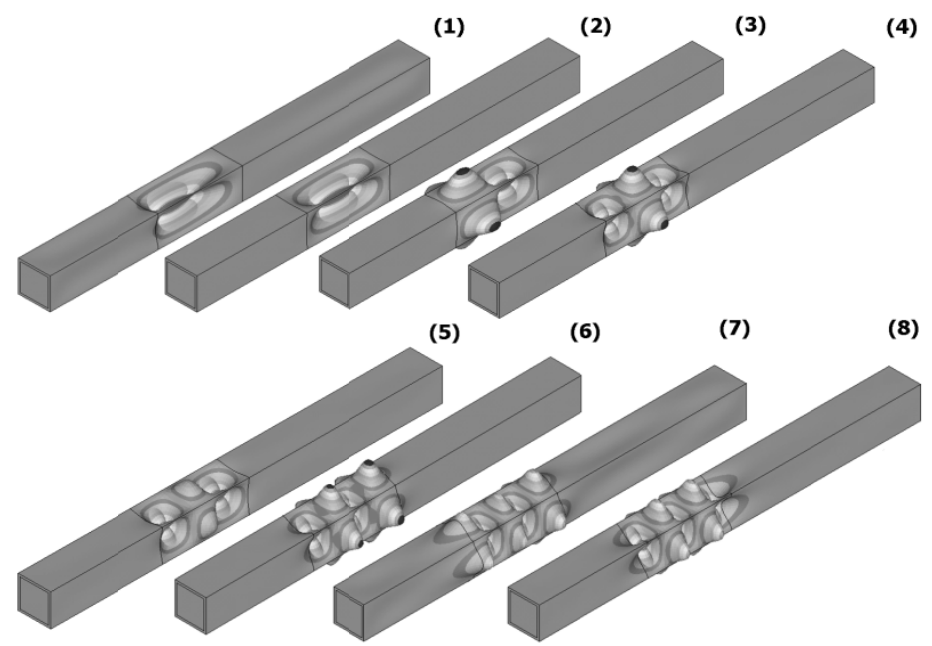

Figure 5-19: Selected coupled modes (structural pattern)

Mapping the modal results onto the experimental pressure spectrum it is found that synchronised modes cover the regions where instabilities occur. The main instability peak is located directly between two synchronised modes at $439 \mathrm{~Hz}$. The secondary instability is 
located around $660 \mathrm{~Hz}$. The predicted instability is shifted more towards the high frequency range than the one obtained during the experiment (at $640 \mathrm{~Hz}$ ). The error can be caused by the assumption of uniform speed of sound within the combustion chamber which is not the case during the experiment. Thermal stresses and pre-stress in the liner are not taken into account, but they may change the mode spectrum as well. The influence of the welds made during the liner manufacturing can have a direct impact on the structural modes, thus also affect the coupled modes.

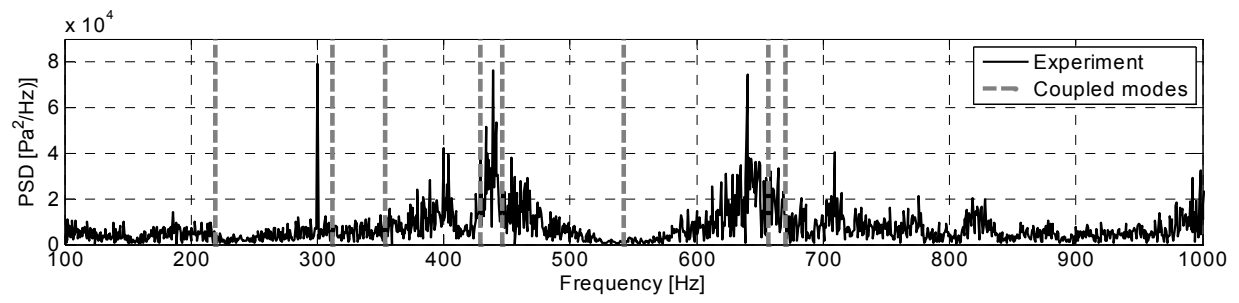

Figure 5-20: Comparison of the experimental results of pressure with data from modal analysis

\subsubsection{Modal ANALYSIS OF Fluistcom}

Due to the same acoustic modes for the Fluistcom and Desire liner configuration, in this section only the coupled modes of the Fluistcom geometry are considered.

In the investigated frequency range, ten coupled modes which present a potentially hazardous pressure pattern are found. In comparison to the Desire configuration, due to lower liner stiffness, the number of possible hazardous frequencies increased about a factor of two. These coupled modes are listed in Table 5-3 and presented in Figure 5-21.

\begin{tabular}{ccccccccccc} 
Number & 1 & 2 & 3 & 4 & 5 & 6 & 7 & 8 & 9 & 10 \\
\hline Eigenfrequency [Hz] & 194 & 207 & 223 & 236 & 249 & 280 & 320 & 370 & $\underline{\mathbf{4 2 8}}$ & $\underline{\mathbf{4 4 5}}$
\end{tabular}

Table 5-3: Eigenfrequencies of the Fluistcom coupled modes

The most energetic modes found during the modal analysis are the synchronised ones at frequencies of $428 \mathrm{~Hz}$ and $445 \mathrm{~Hz}$. These two modes are located in the vicinity of main acoustic instabilities, see Figure 5-23. In contrast to the stiff liner configuration, both are coupled here with unsymmetrical structural modes (8s), see Figure 5-22. However, the 
shape change of the structural modes does not influence the thermo-acoustic instabilities. This behaviour shows that the instabilities are closely related to the location and shape of the acoustic modes and the influence of the structural modes in the investigated case is minor.
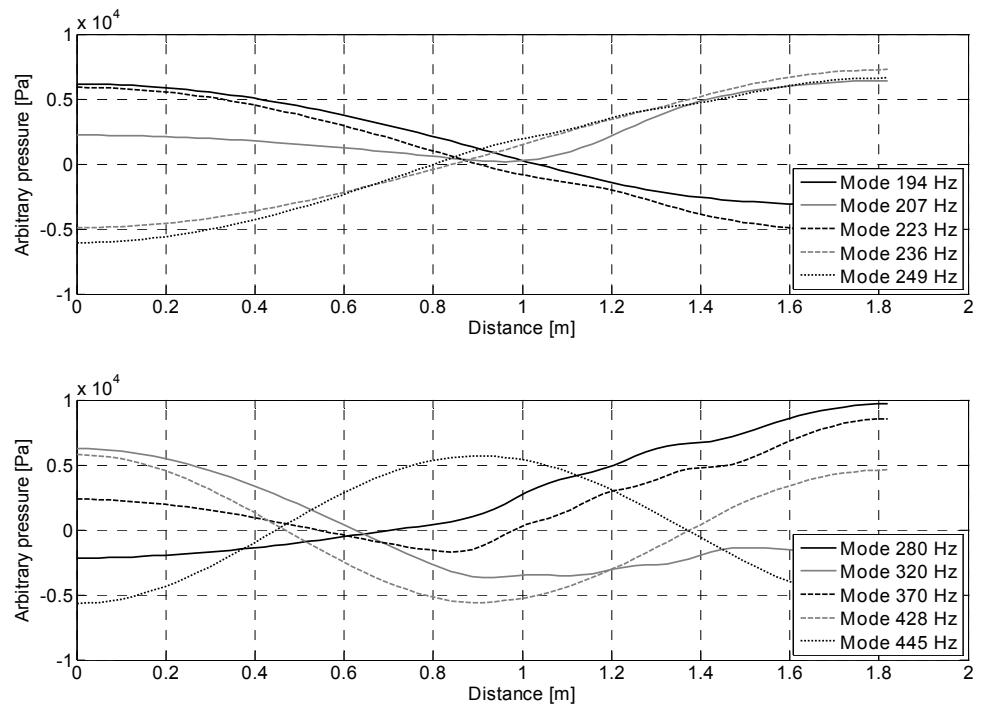

Figure 5-21: Coupled modes (pressure pattern)

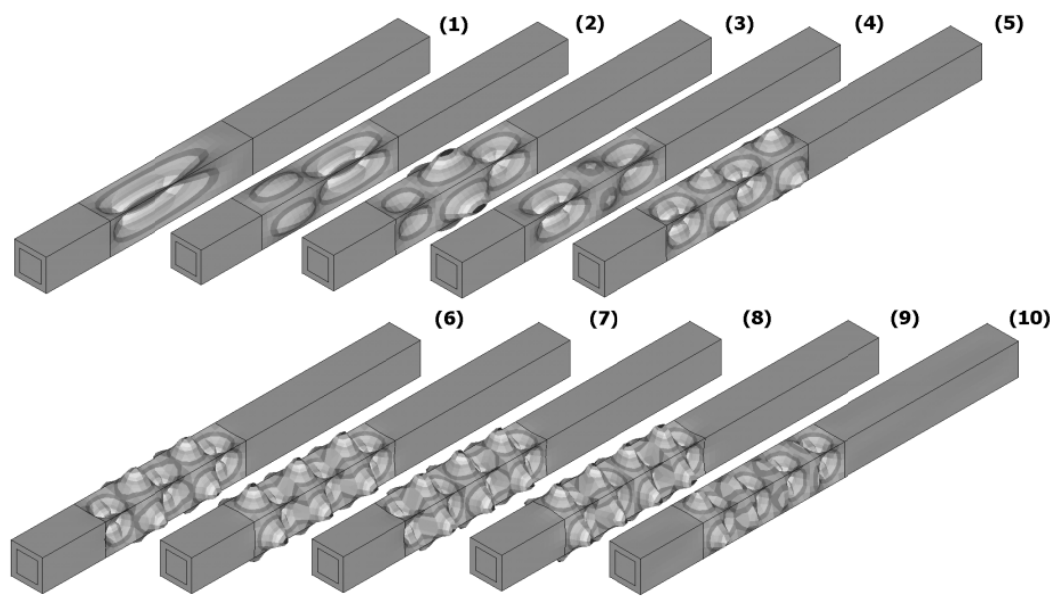

Figure 5-22: Coupled modes (displacement pattern) 

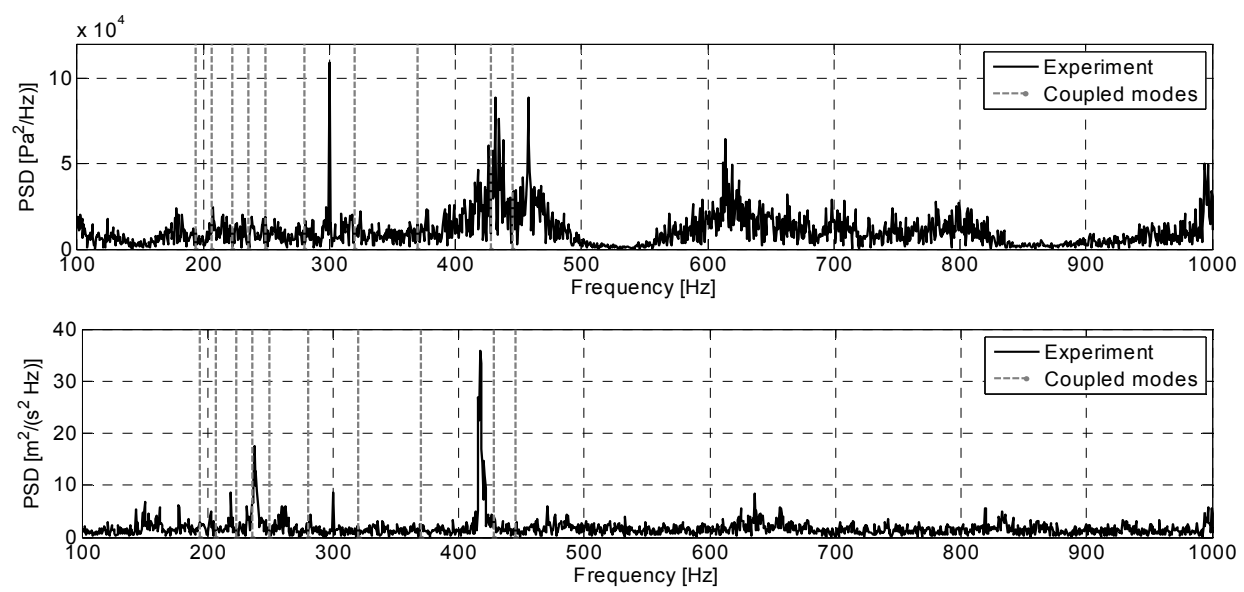

Figure 5-23: Acoustic and coupled modes mapped on pressure (top) and velocity (bottom) spectrum

The rest of the possibly hazardous coupled modes come from the structural vibrations and therefore their effect on the thermo-acoustic instabilities is not significant. Only the mode with eigenfrequency $223 \mathrm{~Hz}$ is created as a result of coupling of the structural mode with the first acoustic mode. Similar to the Desire configuration, the contribution of this mode to pressure instabilities is weak. Nevertheless, due to the very flexible liner configuration, the influence of the first acoustic mode combined with the structural modes (1s-4s), it is visible on the experimental spectrum of the velocity signal, see Figure 5-23 (bottom).

\subsection{Conclusions}

The acousto-elastic and modal analysis are presented in this chapter for two different configurations of the combustion system. For the acousto-elastic calculation a hybrid approach was used. In this approach the pressure field is calculated first in the CFD code. Then the recorded signal is transferred to the FEM code for further analysis. During the FEA interaction between acoustic waves and vibrating walls is observed. In the modal analysis the influence of the coupled structural and acoustic field on the eigenmodes and eigenfrequencies is investigated.

The acousto-elastic analysis shows good agreement of the numerical and experimental results. The amplitude of pressure changes is predicted almost exactly by the numerical scheme, whereas the velocity amplitude shows a minor under-prediction of the experimental results. 
The main frequency of pressure and velocity oscillations is marked by the acousto-elastic model correctly. Next to the forcing frequency visible at all spectra at $300 \mathrm{~Hz}$, the main instabilities are predicted with an error below 3\% for the pressure spectrum and $5 \%$ for the velocity spectrum. Also the secondary instabilities, when they appear, are characterized by a similar error. An exception is the acousto-elastic analysis of the Fluistcom liner configuration without cooling passage, which did not show the main velocity peak in the vicinity of $417 \mathrm{~Hz}$. Therefore, for the simulations with the use of very flexible walls it is preferred to use the model with the cooling passage included. This model gives additional mass, stiffness and damping into the system and these properties can have influence on the system performance.

Due to the implementation of the cooling channel domain into the computation process it was possible to compare pressure signals inside the cooling channel obtained during the numerical and experimental investigation. Also in this case the numerical results predicted the experimental behaviour well.

The difference in the pressure signal between all four acousto-elastic models i.e. Desire and Fluistcom, with and without cooling passage is minor. Increasing the liner flexibility and introducing the cooling passage have impact only on the liner vibration amplitude and frequency.

In case of modal analysis, the most energetic modes which give significant feedback to the system are the synchronised modes. For the combustion chamber configurations investigated in this thesis the synchronised modes are located in the vicinity of the second acoustic mode. 


\section{BACKWARD FACING STEP}

\subsection{INTRODUCTION}

In this chapter the transient and oscillatory heat transfer in a situation typical for a gas turbine combustor is investigated in more detail and in isolation of other difficult to model processes like combustion, swirling flow and acoustics. To this end the combustor is reduced to its most elementary geometry, namely a backward facing step. In a real gas turbine combustion the temperature gradient is vice versa, but it is assumed that for model validation this is not important. The flow over the backward facing step with heat transfer is well documented with experimental data. Experimental data used here are by Vogel \& Eaton, see (Vogel \& Eaton, 1985). Investigated is the simulation of the gas-wall heat transfer in a turbulent flow over the step, with specific attention to the accurate prediction of the flow, thermal boundary layer and resulting wall friction coefficient and heat transfer coefficient.

\subsection{BACKGROUND}

The life time of a gas turbine combustor depends critically on the heat transfer between the liner and the hot combusting flow. An important design characteristic of these combustors is the flow of air and fuel entering at high axial and tangential velocity and small radius the combustor and expanding to larger radii. This determines the flame stabilization, and results in a short flame length. A major complication here is that the hot combusting flow is forced outward due to centripetal forces and is directed towards the liner wall. Hence the hot gases flow at high velocity and thin boundary layer past the liner wall, inducing a high rate of heat transfer, heating the liner to temperatures of $800{ }^{\circ} \mathrm{C}$ or more. The equilibrium liner temperature depends on the balance between heat loss to the cooling air at the cold liner side and heat input at the hot liner side. To complicate the situation, the hot side heat transfer can be enhanced by a transient and oscillating boundary 
layer due to spontaneous oscillations of the main stream hot flow. These can be caused by coupled flame to burner acousto/aero dynamic feedback processes.

The characteristic attribute of the flow over a backward facing step is a separation of the boundary layer at the edge of the step, as presented in (Jovic \& Driver, 1994). Behind the step, as an effect of an adverse pressure gradient, a primary recirculation region is formed, see (Jovic \& Driver, 1995). The length of this region is specified by factors related to the flow properties as well as geometrical dimensions of the sudden expansion channel, as presented in (Rhee \& Sung, 2007) and (Yoshikawa, Suga, \& Ota, 2005), respectively. Typically for the backward facing step, the maximum heat transfer coefficient is observed within the recirculation region close to the reattachment point, see (Valencia, 1997) and (Vogel \& Eaton, 1985). This position and value of the peak in the heat transfer coefficient is correlated in stationary flows to the position and value of the skin friction coefficient. These phenomena are investigated with URANS modelling and application of various turbulence models for stationary and oscillatory flows.

To investigate the influence of the flow and geometrical backward facing step parameters on the variations in the heat transfer and wall friction coefficient, several stationary and transient calculations were performed. The stationary solutions obtained using various turbulent models were compared with experimental data. For stationary calculations the standard k- $\varepsilon$, k- $\omega$ and shear stress transport (SST) models as available in the Ansys CFX code were used. Detailed information about implementation of these models can be found in (CFX-10, 2005). Constants used for all calculations are presented in Table 6-1.

\begin{tabular}{|c|c|c|c|c|c|}
\hline \multicolumn{2}{|c|}{$k-\varepsilon$} & \multicolumn{2}{|c|}{$k-\omega$} & \multicolumn{2}{|c|}{$S S T$} \\
\hline $\mathrm{C}_{\mu}$ & 0.09 & $\beta$, & 0.09 & $\overline{a_{1}}$ & 0.31 \\
\hline $\mathrm{C}_{\varepsilon 1}$ & 1.44 & $\alpha$ & $5 / 9$ & - & - \\
\hline $\mathrm{C}_{\varepsilon 2}$ & 1.92 & $\beta$ & 0.075 & - & - \\
\hline$\sigma_{\mathrm{k}}$ & 1.00 & $\sigma_{\mathrm{k}}$ & 2.00 & - & - \\
\hline$\sigma_{\varepsilon}$ & 1.30 & $\sigma_{\omega}$ & 2.00 & - & - \\
\hline
\end{tabular}

Table 6-1: Model constants

The transient calculations were performed with the turbulence model that presented the best agreement with the experimental results in the stationary situation. The influence of a pulsating inlet velocity and of an oscillatory moving wall on the recirculation region and the rate of heat transfer coefficient was explored. The axial inlet velocity was oscillated in 
the frequency range $10-1000 \mathrm{~Hz}$. The behaviour of a stationary flow over an oscillatory moving wall was explored at a frequency of $10 \mathrm{~Hz}$.

The presentation of the results in this chapter is done on basis of dimensionless numbers, i.e. Stanton number and skin friction coefficient, which are defined in terms of the heat transfer coefficient $(h)$ and wall shear stress $\left(\tau_{\mathrm{w}}\right)$, respectively. The Stanton number, defined as in Equation 6-1 describes the ratio of the heat transferred into the fluid to the thermal capacity of the fluid itself. Whereas the skin friction coefficient, defined by Equation 6-2 is the ratio of the shearing stress exerted by the fluid on the wall surface over which it flows, to the fluid kinetic energy. The heat transfer coefficient is the ratio of heat flux $\left(\mathrm{q}_{\mathrm{w}}\right)$ to temperature difference between temperature at the wall $\left(T_{w}\right)$ and external boundary temperature ( $\left.\mathrm{T}_{0}\right)$, see Equation 6-3.

As already mentioned, for steady flow a correlation between the Stanton number and the skin friction coefficient exists, which might not hold for unsteady flow. Thus in unsteady flows both factors are of interest. In stationary flow this correlation was found to be defined as in Equation 6-5.

Stanton number

Skin friction coefficient

Heat transfer coefficient

Wall shear stress

Reynolds analogy

$$
S t=\frac{h}{C p \cdot \rho \cdot U}
$$

$$
C f=\frac{\tau_{w}}{0.5 \cdot \rho \cdot U^{2}}
$$

$$
h=\frac{q_{w}}{T_{w}-T_{0}}
$$

$$
\tau_{w}=\mu \frac{\partial U}{\partial y}
$$

$$
\frac{C f}{2}=S t \cdot P r^{2 / 3}
$$




\subsection{Numerical Domain}

The geometry of the two-dimensional sudden expansion channel and the location of the coordinate system are depicted in Figure 6-1. Air enters the computational domain upstream of the step. Across the bottom wall, downstream of the step, a constant heat flux is prescribed. The top wall was considered adiabatic and no-slip velocity boundary conditions were employed. In order to obtain the developed turbulent velocity profile upstream of the step, a separate calculation of the developing turbulent flow was performed using a rectangular duct. At the position where numerical data matched with data presented in (Vogel \& Eaton, 1985), i.e. boundary layer thickness $\delta_{99}$ is equal to $4.05 \mathrm{~cm}$, the inlet velocity profile was obtained. However for further calculations, the length of the upstreamstep geometry was reduced to decrease the number of the elements used for the analysis, and as a consequence computational effort. The inlet velocity profile adequate to the shorter upstream-step geometry therefore was used subsequently. Moreover, the inlet velocity profile was compared with data of Oskam, see (Oskam, 1999). Both profiles were in good agreement.

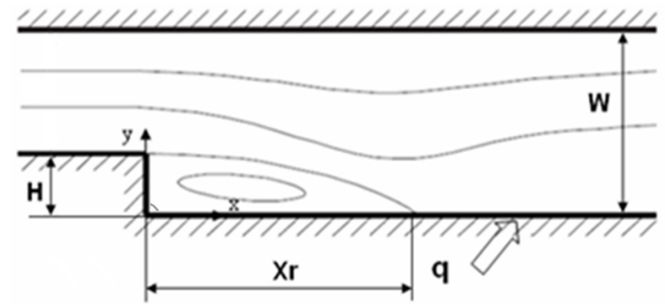

Figure 6-1: Geometry and flow pattern for the backward-facing step calculations

The geometry parameters based on the step height $(\mathrm{H})$ are the following: the length of the domain is $40 \mathrm{H}$, the height upstream of the step is equal to $4 \mathrm{H}$ and downstream $5 \mathrm{H}$ for an expansion ratio $E R=W /(W-H)$ of 1.25 . In the calculations the following value was taken for $\mathrm{H}: \mathrm{H}=3.8 \mathrm{~cm}$, similar to (Vogel \& Eaton, 1985).

The Reynolds number based on the step high (defined as: $R e_{h}=\frac{H U}{v}$ ) during the steady-state calculation was fixed to 28000 and for transient computations with pulsating velocity it varies in the range from 23000 to 33000 .

The CFX code operates always in three dimensional calculations. Thus the numerical model used here for investigation has a third dimension in a spanwise direction with a 
thickness equal to the size of one numerical element. The side walls are periodic. The computational domain consists of 230000 unstructured elements. Most of them are placed in the region close to the step and heated wall. The near-wall region itself is created with the use of prism elements in order to avoid generating highly distorted tetrahedral elements on next to the wall face. The resolution of the grid has a significant effect on the numerical results, thus several trial meshes with different element density and distribution were investigated.

\subsection{Stationary Flow}

Numerical steady-state results with different turbulence models show similar general behavior of the flow over the backward facing step. The skin friction coefficient is predicted negative in the recirculation zone and positive downstream, as shown in Figure 6-2. The reattachment point is defined as the axial position where the skin friction coefficient changes from negative to positive values, see (Le, Moin, \& Kim, 1997) and (Vieser, Esch, \& Menter, 2002). Downstream of the reattachment point, a recovery zone with monotonically increasing skin friction coefficient is observed. The presence of a secondary recirculation bubble, close to the step corner is also visible. The reattachment length based on the step height obtained by the experiments was approximately equal to $6.67 \mathrm{H}$, as shown in (Vogel \& Eaton, 1985). Both the k- $\omega$ and SST turbulence models predicted the reattachment length with an error less than $3 \%$ by respectively $6.74 \mathrm{H}$ and $6.82 \mathrm{H}$. The position of the maximum negative skin friction coefficient was also predicted correctly, as well as its magnitude. More significant errors are observed for the k- $\varepsilon$ model. The reattachment point is predicted about one step height closer to the step, at a position of $5.66 \mathrm{H}$. The secondary recirculation bubble which appears close to the step corner is hardly visible despite the grid resolution. The overall error it is of the order of $15 \%$ (consistent with other numerical investigations see (Thangam \& Speziale, 1992)), but it can be much higher downstream in the recovery region. All models presented significantly underestimation of the skin friction coefficient in the recovery zone. This behaviour is observed also by other researchers, see (Vieser, Esch, \& Menter, 2002). The explanation for this is at the moment unclear, which points to more research necessary at this topic.

The behavior of the Stanton number is generally opposite to the skin friction coefficient. The investigated models predicted the same, typical behavior. A sudden drop of the heat transfer coefficient just downstream of the step is followed by a sharp increase till its maximum value near the reattachment point and by a monotonic decrease further downstream in the recovery region. The maximum value of the heat transfer coefficient is almost twice the value in the recovery zone downstream, where the flow has developed 
parallel to the wall again. Both, k- $\omega$ and SST models predicted almost exactly in line with experiments the position of the maximal Stanton number peak, as illustrated in Figure 6-3. The SST model also predicted correctly the peak magnitude, whereas the other models gave a significant underestimation. The k- $\varepsilon$ model Stanton number values seem to be shifted, similar to the skin friction coefficient results, upstream.

In steady flow conditions, the heat transfer coefficient can be correlated to the skin friction coefficient and the level of turbulence intensity. This correlation, the Reynolds analogy, is given in Equation 6-3. The flow over the backward facing step just behind the step is almost stagnant and not very turbulent. Downstream of the step, near the heated wall, the flow turbulence suddenly increases to its maximal value up to the point of maximal heat transfer coefficient. In the recovery region, the turbulence level decreases slowly. The behaviour of the Stanton number is very similarly increasing to a maximum and decreasing to a stationary value over an identical range in $\mathrm{x} / \mathrm{H}$, see Figure 6-4.

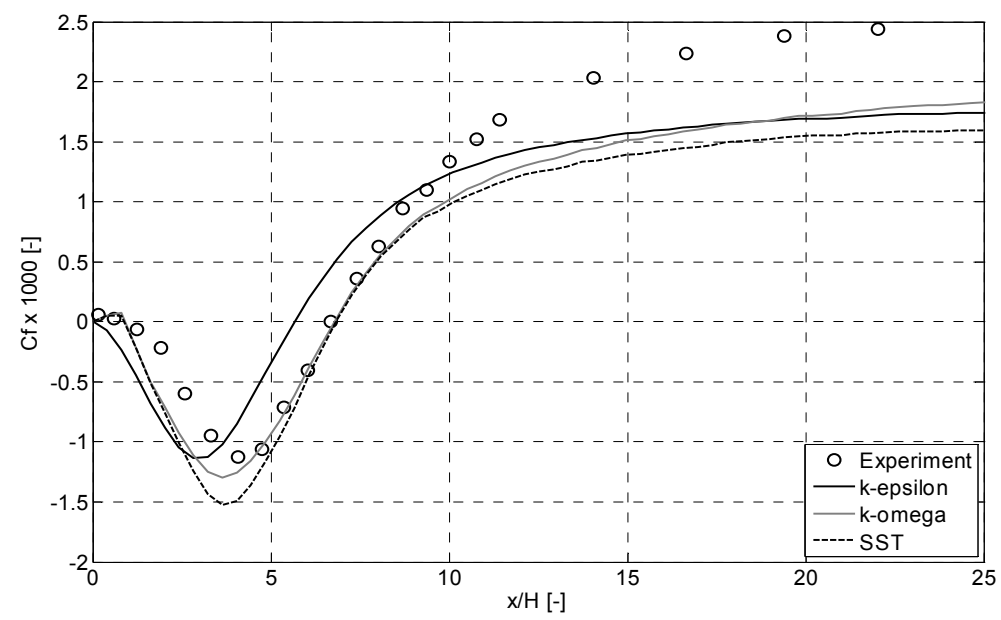

Figure 6-2: Comparison of the skin friction coefficient for the $k$ - $\varepsilon, k$ - $\omega$ and SST turbulence model 


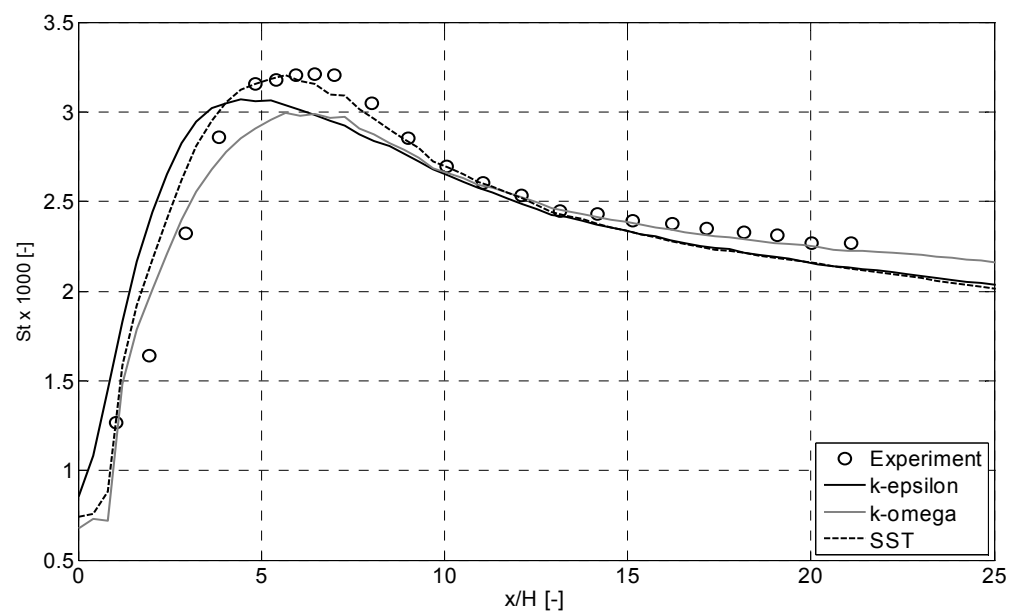

Figure 6-3: Comparison of the Stanton number for the $k-\varepsilon, k-\omega$ and SST turbulence model

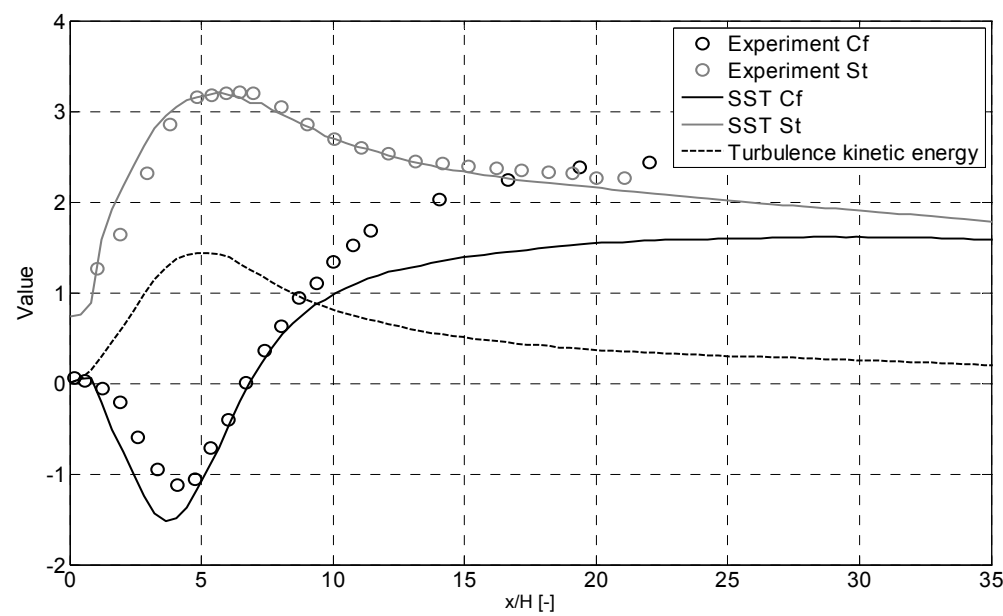

Figure 6-4: Turbulence kinetic energy (at position $0.005 \mathrm{~m}$ from the bottom wall), the skin friction coefficient and Stanton number profiles for the SST turbulence model

The locations of the maximum peaks in the Stanton number and turbulence level are very close: about 0.7 of the step height. This suggests that the Stanton number and the turbulence level are very much correlated. This correlation between the skin friction coefficient and turbulence cannot be observed, as the former changes sing at the edge of the recirculation bubble. Therefore the Reynolds analogy does not hold in the recirculation region, in which poor agreement between the Stanton number and skin friction coefficient exists. 
Downstream in the recovery region $(x / H>30)$, the skin friction coefficient and the Stanton number behave as in the turbulent flat plate solution and the validity of the Reynolds analogy is recovered. The poor prediction of the skin friction coefficient in the recovery region by all turbulent models moved the position of Reynolds analogy application downstream of the investigated channel.

\subsection{Transient Flow - Inlet Velocity Pulsation}

In this section transient calculations performed with a pulsating inlet velocity are presented. The turbulence model used was the SST model which gave the best prediction in the steady-state configuration. In order to investigate the influence of the pulsating flow on the recirculation region and the heat transfer coefficient, several calculations were performed at different forcing frequencies: 10, 100, 400 and $1000 \mathrm{~Hz}$. The corresponding Strouhal number, defined as $S r=f \cdot H / U$ ranges from 0.035 for the $10 \mathrm{~Hz}$ excitation to 3.5 for the excitation equal to $1000 \mathrm{~Hz}$. The forcing amplitude of pulsation was fixed to $20 \%$ of the initial velocity profile. The Reynolds number based on the step height is varied in the range of 22000 - 33000 . The calculations time step has been adjusted depending on the frequencies and 10 or 20 samples per cycle were taken. The mean values of the skin friction coefficient and Stanton number are obtained by averaging the instantaneous values over one cycle. Other geometrical and boundary parameters, as well as grid resolution were undisturbed. Unfortunately no experimental data are available for the unsteady case and the steady-state experimental data are plotted just for comparison.

The pulsations of the inlet velocity caused a variation in the reattachment length. In all investigated cases the primary recirculation bubble increases together with the decreasing inlet velocity. This behavior was observed also by (Valencia, 1997). The secondary bubble placed near the step corner is increasing together with the primary recirculation region, as illustrated in Figure 6-5.

As shown above the length of the recirculation region significantly affects the skin friction coefficient and Stanton number. Thus both profiles oscillate according to the recirculation bubble configuration. In all cases the instantaneous maximum negative peak for $\mathrm{Cf}$ was several times higher than in the steady-state calculations, as presented in Figure 6-6. Also its axial position varied together with the changes in the recirculation zone. The instantaneous changes in the magnitude and location of the maximum skin friction coefficient did not affect significantly its mean value for frequencies of $100 \mathrm{~Hz}$ and higher. For the Strouhal numbers investigated in the range $0.35-3.5$, the mean maximum peak in the skin friction coefficient has almost the same magnitude and position as the one in the 
steady-state calculations. The main average trend is as well in good agreement with the steady-state results for frequencies above $100 \mathrm{~Hz}$, as shown in Figure 6-7.
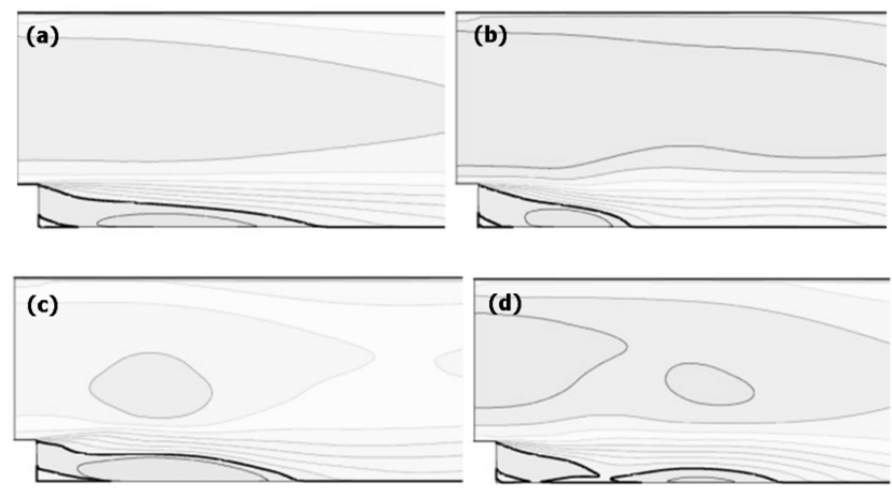

Figure 6-5: Axial velocity profiles (zero axial velocity marked by bold line; inside the bubbles velocity has negative direction) of the SST model for the steady state calculations (a), and for pulsations with the excitation frequency $10 \mathrm{~Hz}$ : phase $5 / 10 \pi(b)$, phase $13 / 10 \pi(c)$, phase $1 / 10 \pi(d)$

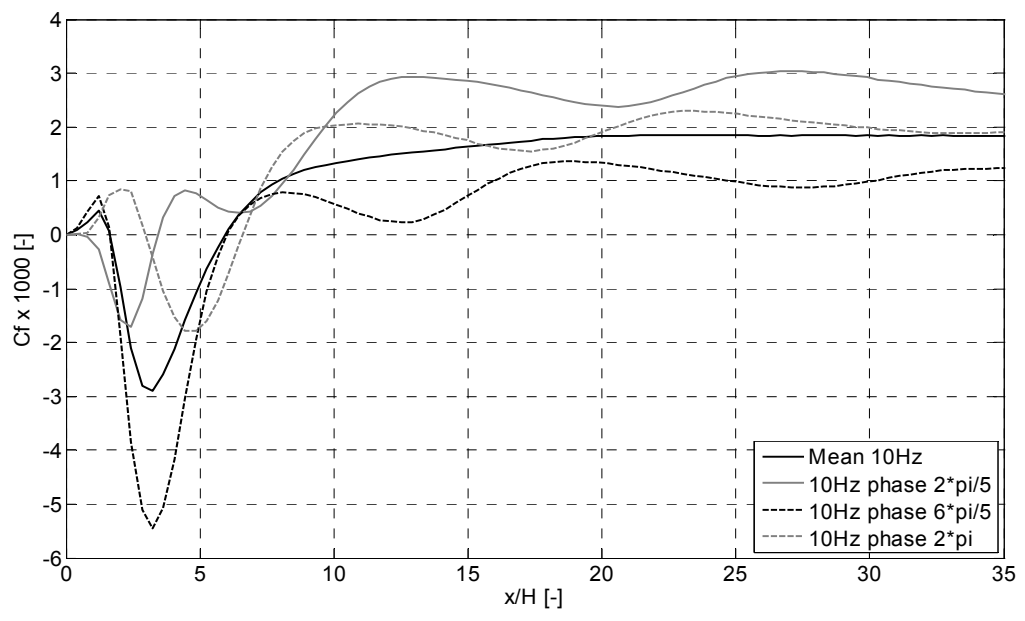

Figure 6-6: Transient results of the skin friction coefficient -10 Hz case

In the $10 \mathrm{~Hz}$ frequency case however, the mean profile differs significantly from the steadystate results. The mean value of the maximum negative peak in the skin friction coefficient is two times higher and shifted upstream of the domain in comparison to the steady-state profile, as depicted in Figure 6-7. Thus the mean recirculation region is also shorter. The 
secondary bubble formed near the step corner with positive axial velocity is clearly visible. In the recovery region, the mean skin friction coefficient profile presents similar behavior as in the steady-state case. This behavior of the mean skin friction coefficient profile can be explained as a result of significant changes in the instantaneous velocity profiles. The secondary bubble formed near the step has a major influence on the skin friction coefficient. During the excitation the secondary bubble increases together with the decreasing of the inlet velocity till its maximal length. At this point, the velocity starts to increase again. As a result of the time delay between changes in the inlet velocity profile and response of the recirculation region and the secondary bubble, the recirculation zone is broken up in two separate parts. Thereafter, the part close to the step behaves like the main recirculation region. Inside this region the secondary bubble is formed again. The part far from the step disappears with time advancing and a whole cycle is repeated again. During the $10 \mathrm{~Hz}$ excitation cycle, it has not been possible to specify exactly the instantaneous reattachment point because of the simultaneous presence of two separate recirculation bubbles in the near wall region. A similar behavior was observed in low frequency structures in a DNS of the flow over the backward facing step without heat transfer by (Le, Moin, \& Kim, 1997).

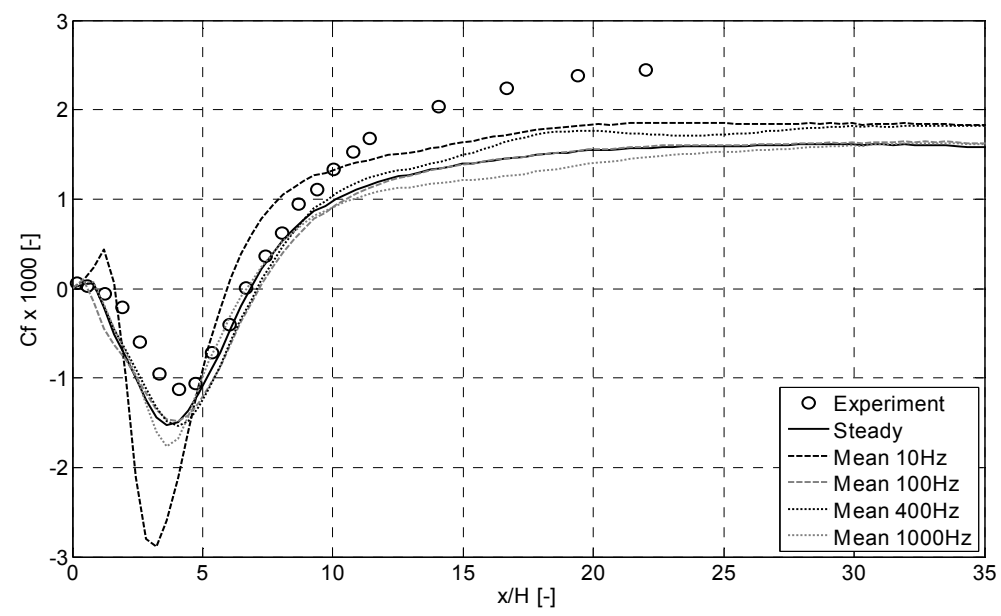

Figure 6-7: Mean results of the skin friction coefficient

For the $10 \mathrm{~Hz}$ excitation, the transient changes in the heat transfer coefficient are significant. The maximum peak in the Stanton number corresponds exactly to the maximum peak of the skin friction coefficient. Due to the recirculation bubble phenomena described above, the maximum peaks in skin friction and heat transfer are moving up and down, 
backward and forward, depending on the recirculation zone position, as presented in Figure 6-8. As a consequence, the mean Stanton number profile for $10 \mathrm{~Hz}$ excitation frequency is significantly changed, see Figure 6-9.

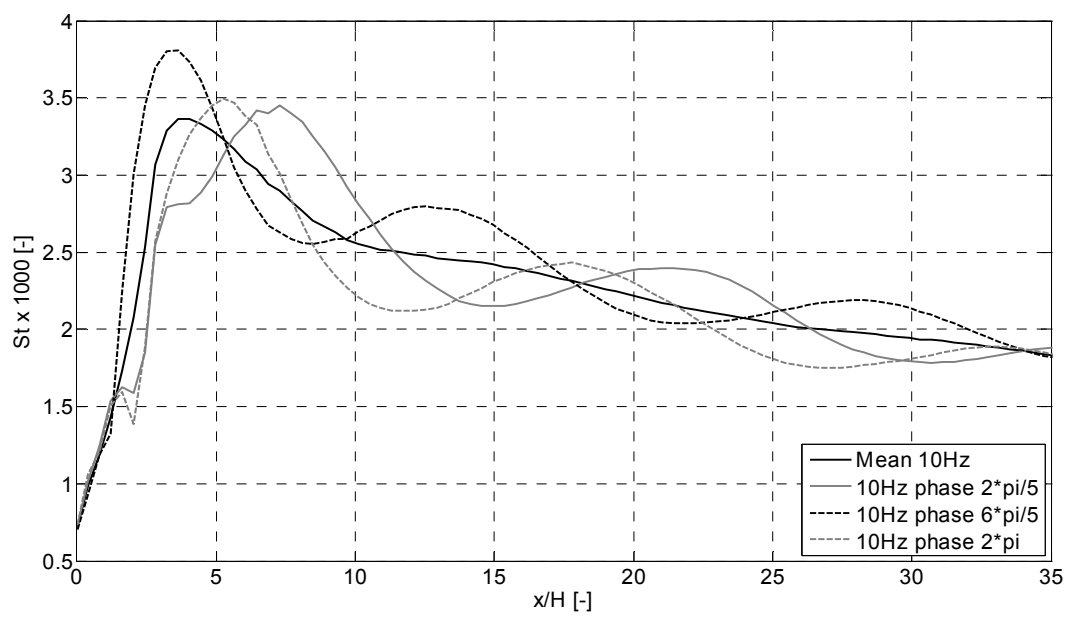

Figure 6-8: Transient results of the Stanton number - $10 \mathrm{~Hz}$ case

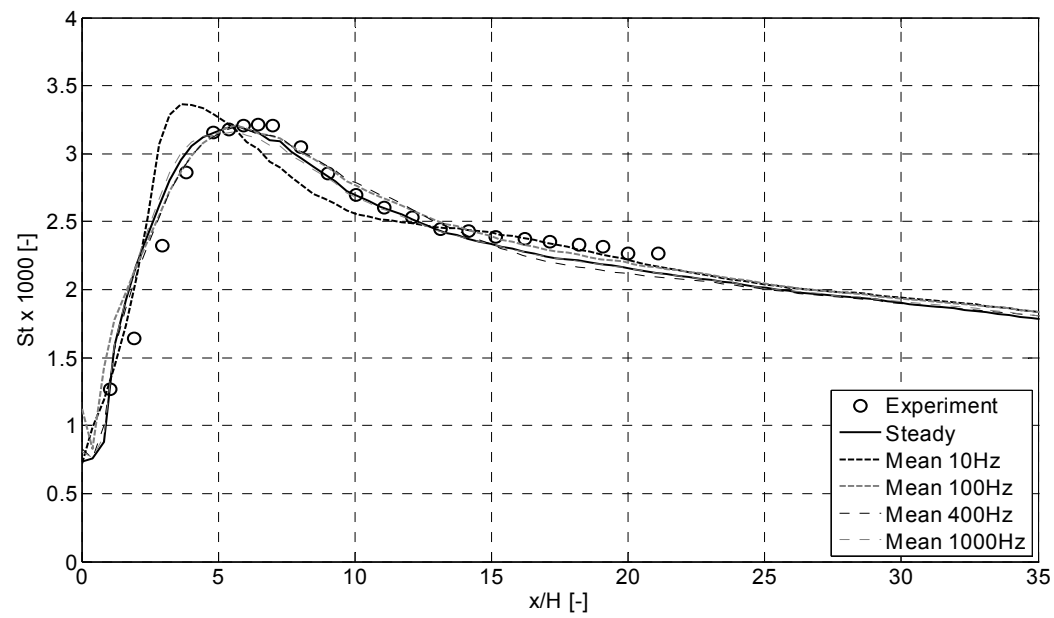

Figure 6-9: Mean results of the Stanton number

The maximum peak in mean Stanton number is higher and shifted upstream. The mean temperature field near the wall is however little affected by the fast, transient changes in the velocity field at frequencies of $100 \mathrm{~Hz}$ and above. Hence, the mean values of the Stanton 
number profiles agree well with steady-state numerical results for high frequency, see Figure 6-9.

\subsection{Transient Flow - Oscillating Wall}

The transient calculations of the flow over the backward facing step with the oscillating bottom wall are done for the same configuration of the domain as for investigated in earlier cases. The inlet velocity profile is undisturbed. Instead, the heated bottom wall is oscillating with a frequency equal to $10 \mathrm{~Hz}$. The maximum amplitude of the vertical displacement is equal to $2 \mathrm{~mm}$. This leads to a variation of the expansion ratio, based on the geometrical dimensions of 1.24-1.26. The time step was fixed to $5 \mathrm{~ms}$ and 10 samples per cycle were collected. The mean values of the skin friction coefficient and Stanton number were obtained by averaging results over one cycle. Other flow and geometrical properties are left unchanged. For calculations with the moving wall, a model with a moving grid was used. The total number of elements was kept constant. The region near the heated wall was made with the use of prism elements, thus any changes in the domain during wall movement did not cause significant element distortion.

The instantaneous changes in the position of the bottom wall have a significant effect on the reattachment region. The length of the recirculation zone varies according to the changes in the step height, as shown in Figure 6-10. When the heated wall is moving upward and the step height is decreasing, the reattachment point is moving downstream of the domain. When the step height is increasing, the reattachment region is decreasing. The instantaneous maximum negative peak in the skin friction coefficient is about twice as high as the steady-state value. The peak in the skin friction coefficient is moving downstream and upstream depending on the transient reattachment region length. However, the changes are not as significant as in the case of the pulsating inlet velocity. The secondary bubble is not growing to dimensions which could threaten the breaking up of the recirculation region. The mean velocity profile is not much different from the steady-state one, only the maximum mean skin friction coefficient peak is higher than the one observed during steady-state calculations. The mean length of the recirculation region is the same as during the steady-state investigation. Also the behavior of the mean skin friction coefficient profile in the recovery region matches the steady results. 


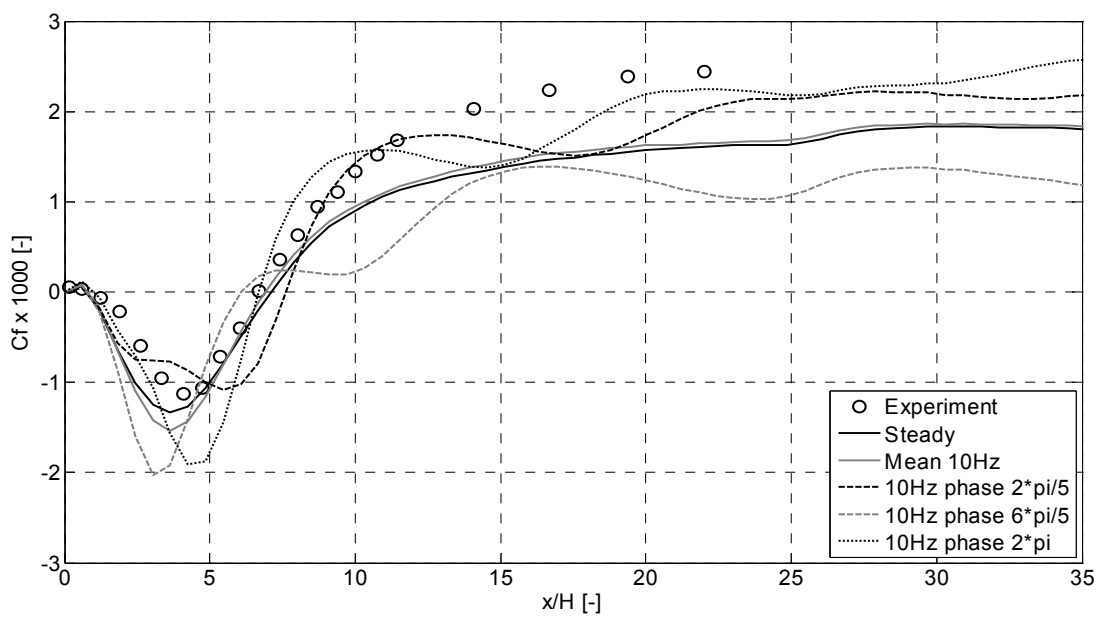

Figure 6-10: Instantaneous and mean values of the skin friction coefficient during calculations with moving wall

The Stanton number follows the changes in the skin friction coefficient, as illustrated in Figure 6-11. Because of the small frequency of excitation, the heat transfer is affected by changes in the recirculation length. The instantaneous, maximum peak in the Stanton number is located at the position which corresponds to the transient location of the maximum peak of the skin friction coefficient. However, the general increasing or decreasing tendency of the heat transfer coefficient is affected by a certain time delay. The location of maximum value of the Stanton number is moved towards the location of maximum value of the skin friction coefficient. The time delay is equal approximately to 30 ms.

These changes are not high enough to change significantly the mean Stanton number profile. Only minor changes in the vicinity of the maximum peak of Stanton number are observed. At this position mean value of the transient Stanton number is slightly bigger with respect to the steady-state profile. It can be concluded, that despite the high amplitude of vibration of $2 \mathrm{~mm}$, the bottom wall does not have a significant effect on the mean skin friction coefficient and heat transfer. 


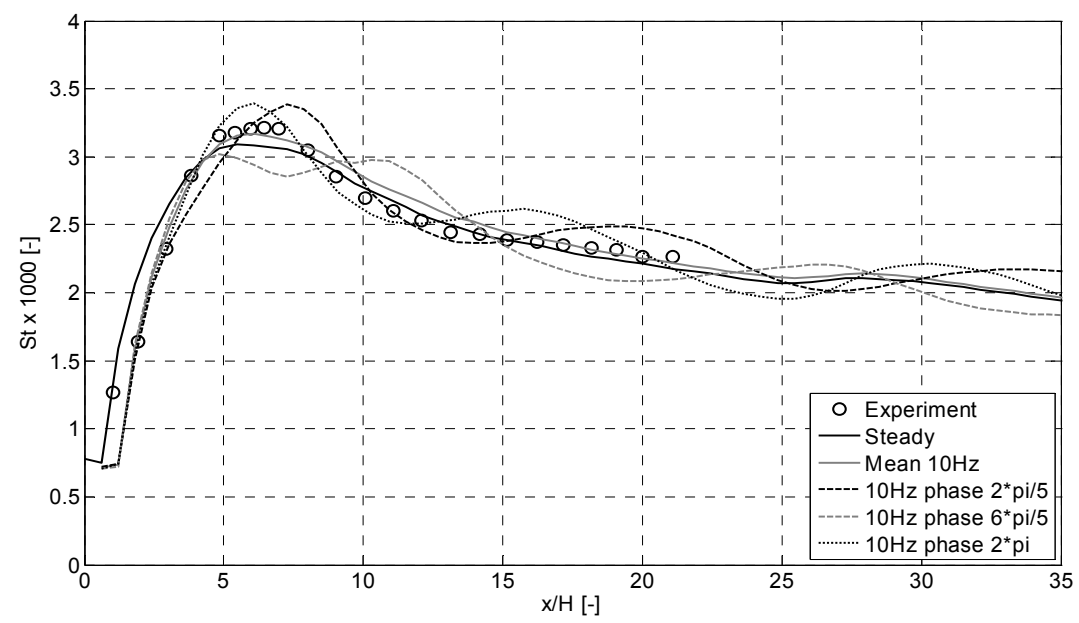

Figure 6-11: Instantaneous and mean values of the Stanton number during calculations with moving wall

\subsection{CONCLUSIONS}

For steady inlet flow conditions, the k- $\omega$ and SST turbulent models investigated showed good agreement with the experimental data on the skin friction coefficient and the Stanton number. The k- $\varepsilon$ model, however, revealed a $15 \%$ under-prediction of the reattachment length at locally even much more. The $\mathrm{k}-\omega$ and SST turbulence models presented an error smaller than $3 \%$.

The effect of the pulsating inlet velocity is especially significant at the excitation frequency of $10 \mathrm{~Hz}$. In this case the secondary recirculation bubble formed in the corner of the step is broken up in two parts. In all cases the instantaneous maximal negative skin friction coefficient was several times bigger than in the steady-state calculations. This means the wall is more exposed to temperature, increasing the risk of fatigue. At frequencies above $100 \mathrm{~Hz}$, the effect of a mean Cf and St is absent.

The variations in the wall position affected the transient values of both the skin friction coefficient and the Stanton number, but the time mean values were left almost unchanged at frequencies above $100 \mathrm{~Hz}$. Only in the case of the $10 \mathrm{~Hz}$ excitation frequency minor changes were observed. 
The changes in the wall position affected the instantaneous values of the skin friction coefficient and the Stanton number. However, the influence of those changes on the position of the main peaks as well as on the main profiles is not significant.

A certain time delay of the peak position between instantaneous profiles of the skin friction coefficient and the Stanton number was observed. 



\section{CONCLUSIONS AND RECOMMENDATIONS}

The focus of this thesis is on the mutual interaction between thermo-acoustic instabilities and wall vibrations in modern gas turbine combustors. Experimental and numerical investigations were performed to have the insight and possibility to predict the behaviour of the combustion system working in lean premixed combustion regime. Of the highest importance was to predict the frequency spectrum of both: thermo-acoustic instabilities and vibrations of the liner. The strong coupling between these phenomena may lead to gas turbine engine destruction. The multi-disciplinary character of the conducted investigations has reflection in the numerical tools and techniques used for study. To capture all phenomena interacting together, different numerical approaches were used. The Computational Fluid Dynamics and structural Finite Element Analysis were coupled together in direct (Fluid-Structure Interaction analysis) and indirect (Acousto-Elastic analysis) manner. They were supported additionally by separate CFD computations of the combustible flow and modal analysis of the structural liner. The results, obtained using numerical approach, were validated against the experiment. Different operating points and liner configurations were analysed. The conclusions $(\bullet)$ and recommendations $(\circ)$ obtained during these studies are organised in two main sections i.e. experimental measurements and numerical techniques.

\section{EXPERIMENTAL MEASUREMENTS}

- In the current configuration of the setup, the thermo-acoustic instabilities are located around the second acoustic mode of the combustion chamber.

- The spectrum of the pressure fluctuations is similar for both liner configurations. However, the thermo-acoustic instabilities in case of the flexible liner configurations are moved slightly to lower frequencies $(405-431 \mathrm{~Hz})$ with 
comparison to the stiff liner $(433-458 \mathrm{~Hz})$. This behaviour suggests that the flexibility of the liner may have influence on the thermo-acoustic instabilities frequency but in the current setup configuration this effect is minor. Furthermore, change in frequency of thermo-acoustic instabilities at given liner configuration suggests that thermo-acoustic instabilities can be coupled to different structural modes located in the vicinity of acoustic mode.

- The vibration characteristics of the liner follow the pressure spectrum. In case of the flexible liner also peak around first acoustic eigenfrequency of the combustion chamber is visible. This is an indication that for a more flexible liner, the first acoustic eigenfrequency of the system might take a lead of the thermo-acoustic instabilities.

- The acoustic spectrum in the cooling passage resembles the pressure spectrum inside the combustion chamber, but it is several $\mathrm{dB}$ smaller. The acoustic signal measured there comes from both: walls vibrations and channels connection. The undulation of the signal is made by obstacles located there for data acquisition.

- The flame transfer function reconstructed from factorized transfer functions shows similar behaviour for different operating points and geometries. However, here the power to pressure ratio is kept almost constant at $83 \mathrm{~kW} / \mathrm{bar}$.

- Further investigations of the interaction between thermo-acoustic instabilities and liner vibrations should be conducted at modified liner. The new liner should have lower stiffness. Preferably, since the flexible section is located far from the flame and it gives only a minor influence to the flame behaviour, the new flexible section should be located in the vicinity of the flame. Otherwise, the liner should have uniform thickness (approximate $2 \mathrm{~mm}$ ) over its entire length.

- If the configuration presented above due to the construction limits cannot be achieved, a new atmospheric test rig with flexible walls can be applied for this type of investigations. Here, the stiffness of the liner can be adjusted by the external supports. This way the effect of vibrating liner and modes at which it vibrates on the flame characteristics can be investigated.

\section{NuMERICAL TECHNIQUES}

- All investigated here computational models presents a good agreement with experimental data. Fluid-structure interaction and acousto-elastic analysis allows predicting whether and at which frequency, for a given operating conditions, the 
thermo-acoustic instabilities occur, with recorded error below $6 \%$. Thanks to direct linking between wall vibrations with acoustic field and combustion process (FSI) or with acoustic field only (AE), the effect of vibrating walls on the combustion system may be observed.

- Both models show new directions of solving coupled fields problem in gas turbine system. In the fluid-structure interaction model an interface connection between CFD and FEA is created for data transfer every time step, whereas the AE model uses a hybrid approach, where information is exchanged after the CFD run is finished.

- The CFD analysis is crucial for the accuracy of the coupled system investigation. Any errors which appear here have a major impact on the coupled system study.

- Due to a short computed physical time (around $80 \mathrm{~ms}$ ) and acoustic wave dissipation, the amplitudes of instabilities resolved by the CFD and FSI models are under-predicted. Even though, the hazardous frequencies are marked correctly. This suggests a linear behaviour of the system.

- In the aftermath of abovementioned, the liner velocities are also under-predicted.

- Similar results of FSI one-way and two-way interaction are obtained. The one-way interaction model profits from shorter calculation time, however, the two-way interaction model is more sensitive to the instabilities, especially in case of more flexible liner. Therefore, for computations of a fluid-structure interaction with very flexible walls or in case of nonlinearities in the system (e.g. for limit cycle oscillations) the two-way interaction appears to have a priority.

- Introduction of the cooling passage to FSI analysis is possible. However, the benefits of more precise results may not be pay off by the costs of the computational time.

- The prediction of the instability frequencies by the acousto elastic model (indirect coupling) is done with similar errors as during the FSI computations. Only exception is a velocity spectrum of the flexible liner configuration without cooling passage where the instabilities are hardly visible at frequency observed during experiments. Therefore application of this model to a very flexible liner configuration without cooling passage should be done carefully.

- The amplitude of pressure changes is predicted almost exactly by the numerical scheme, whereas the velocity amplitude shows a minor under-prediction of the experimental results.

- Modal analysis of the combustion chamber geometry will give the hazardous frequencies at which the instabilities may occur. However, separate CFD, FSI or $\mathrm{AE}$ analysis must be performed in order to investigate whether at given operating point the instabilities are built up. 
- Inlet velocity pulsations at various frequencies and amplitude equal to $20 \%$ of the mean velocity investigated during the BFS analysis have shown only minor effect on the time average heat transfer (except of $10 \mathrm{~Hz}$ excitation frequency). Also wall vibrations with amplitude of $2 \mathrm{~mm}$ and frequency equal to $10 \mathrm{~Hz}$ did not disturb the mean Stanton number.

- Both fluid-structure interaction and acousto-elastic analysis may be used by industry to reduce the effect of the thermo-acoustic instabilities on the system, however further investigations should be done to reduce and optimize the required calculation time.

- Further investigations with temperature profile transferred between codes should be done in order to capture well modes of liner vibrations during FSI and AE analysis.

- The acousto-elastic model represents only the one-way coupling between acoustic waves and flame i.e. it is not possible to modify the pressure signal exported from CFD by incoming waves. In order to improve the AE model, a two-way data transfer from walls to pressure field imported from CFD must be done. The pressure boundary conditions must be a function of the incoming wave at a certain location in the combustion chamber.

- Calculations of the coupled problems which involve acoustics should be performed with non-adiabatic walls to reduce the effect of an over-predicted, uniform temperature distribution along the combustion chamber. This may disturb the propagation of acoustic waves in the system.

- The SAS turbulent model or even LES to capture well the instabilities in the system are advised.

- Calculations with CFL number above 10 have shown a good prediction of thermoacoustic instabilities. However, the CFL number in range of 0.1 is necessary for fundamental investigations of the instabilities which may occur in the system. This restricts the computations to very small time step and elements size, but numerical dispersion and dissipation of acoustic wave will be reduced significantly.

- Extending the computational time to few seconds should improve the numerical results. The same applies to the temperature profile of the liner which can be exported into the FEA. Both however, will extend the calculation time significantly. 


\section{ROMAN}

$\begin{array}{cll}A & \text { Wave amplitude } & {[\mathrm{Pa}]} \\ \boldsymbol{C} & \text { Damping matrix } & {[\mathrm{kg} / \mathrm{s}]} \\ C_{f} & \text { Skin friction coefficient } & {[-]} \\ D a & \text { Damköhler number } & {[-]} \\ E & \text { Young's modulus } & {[\mathrm{Pa}]} \\ \boldsymbol{F} & \text { Force matrix } & {[\mathrm{N}]} \\ H_{f} & \text { Flame transfer function } & {[-]} \\ \boldsymbol{K} & \text { Stiffness matrix } & {[\mathrm{N} / \mathrm{m}]} \\ M & \text { Acoustic source term } & {\left[\mathrm{kg} /\left(\mathrm{s}^{2} \mathrm{~m}^{3}\right)\right]} \\ \boldsymbol{M} & \text { Mass matrix } & {[\mathrm{kg}]} \\ M a & \text { Mach number } & {[-]} \\ \boldsymbol{P} & \text { Pressure vector } & {[\mathrm{Pa}]} \\ P r & \text { Prandtl number } & {[-]} \\ \dot{Q} & \text { Power } & {[\mathrm{W}]} \\ \boldsymbol{R} & \text { Coupling matrix } & {\left[1 / \mathrm{m}^{2}\right]} \\ R e & \text { Reynolds number } & {[-]} \\ S & \text { Area } & {\left[\mathrm{m}^{2}\right]} \\ S c & \text { Schmidt number } & {[-]} \\ S r & \text { Strouhal number } & {[-]} \\ S t & \text { Stanton number } & {[-]}\end{array}$




\begin{tabular}{|c|c|c|}
\hline$T$ & Temperature & {$[\mathrm{K}]$} \\
\hline$U$ & Velocity & {$[\mathrm{m} / \mathrm{s}]$} \\
\hline $\boldsymbol{U}$ & Displacement vector & {$[\mathrm{m}]$} \\
\hline$V$ & Volume & {$\left[\mathrm{m}^{3}\right]$} \\
\hline$W$ & Width of a channel & {$[\mathrm{m}]$} \\
\hline$X r$ & Reattachment length & {$[\mathrm{m}]$} \\
\hline$Y$ & Mass fraction & {$[-]$} \\
\hline$c$ & Speed of sound & {$[\mathrm{m} / \mathrm{s}]$} \\
\hline$c_{p}$ & Specific heat at constant pressure & {$[\mathrm{J} /(\mathrm{kg} \mathrm{K})]$} \\
\hline$c_{v}$ & Specific heat at constant volume & {$[\mathrm{J} /(\mathrm{kg} \mathrm{K})]$} \\
\hline$f$ & Frequency & {$[\mathrm{Hz}]$} \\
\hline$f_{C}$ & Cut-off frequency & {$[\mathrm{Hz}]$} \\
\hline$h$ & Enthalpy & {$[\mathrm{J} / \mathrm{kg}]$} \\
\hline$h$ & Thickness & {$[\mathrm{m}]$} \\
\hline$h$ & Heat transfer coefficient & {$\left[\mathrm{W} /\left(\mathrm{m}^{2} \mathrm{~K}\right)\right]$} \\
\hline$k$ & Turbulence kinetic energy & {$\left[\mathrm{m}^{2} / \mathrm{s}^{2}\right]$} \\
\hline$k$ & Wave number & {$[1 / \mathrm{m}]$} \\
\hline$m, n$ & Number of half acoustic waves in $\mathrm{x}, \mathrm{y}, \mathrm{z}$ direction & {$[-]$} \\
\hline$\dot{m}$ & Mass flow rate & {$[\mathrm{kg} / \mathrm{s}]$} \\
\hline$n$ & Normal direction vector & {$[-]$} \\
\hline$p$ & Pressure & {$[\mathrm{Pa}]$} \\
\hline$q$ & Heat flux & {$\left[\mathrm{W} / \mathrm{m}^{2}\right]$} \\
\hline$r$ & Characteristic impedance & {$\left[\mathrm{kg} /\left(\mathrm{m}^{2} \mathrm{~s}\right)\right]$} \\
\hline$t$ & Time & {$[\mathrm{s}]$} \\
\hline$t$ & Cauchy stress & {$[\mathrm{Pa}]$} \\
\hline$t_{p}$ & Plate thickness & {$[\mathrm{m}]$} \\
\hline$u$ & Velocity & {$[\mathrm{m} / \mathrm{s}]$} \\
\hline$w_{p}$ & Plate width & {$[\mathrm{m}]$} \\
\hline
\end{tabular}




\section{GREEK}

$\Gamma \quad$ Mesh stiffness

$\left[\mathrm{m}^{2} / \mathrm{s}\right]$

$\Delta t \quad$ Discrete time step

[s]

$\Delta x \quad$ Characteristic grid size

[m]

$\phi \quad$ Equivalence ratio

[-]

$\alpha \quad$ Thermal diffusivity

$\left[\mathrm{m}^{2} / \mathrm{s}\right]$

$\gamma \quad$ Specific heat ratio

$[-]$

$\gamma \quad$ Mass of a plate per unit area

$\left[\mathrm{kg} / \mathrm{m}^{2}\right]$

$\delta \quad$ Displacement

[m]

$\varepsilon \quad$ Turbulence eddy dissipation

$\left[\mathrm{m}^{2} / \mathrm{s}^{3}\right]$

$\lambda$ Thermal conductivity

$[\mathrm{W} /(\mathrm{m} \mathrm{K})]$

$\lambda \quad$ Wave length

[m]

$\lambda \quad$ Air factor

$[-]$

$\lambda_{l m} \quad$ Tabularized factor for modal analysis

[-]

$\mu \quad$ Dynamic viscosity

$[\mathrm{kg} /(\mathrm{m} \mathrm{s})]$

$\mu_{\text {eff }} \quad$ Effective viscosity

$[\mathrm{kg} /(\mathrm{m} \mathrm{s})]$

$\mu_{t} \quad$ Turbulence viscosity

$[\mathrm{kg} /(\mathrm{m} \mathrm{s})]$

$v \quad$ Poisson's ratio

[-]

$v \quad$ Kinematic viscosity

$\left[\mathrm{m}^{2} / \mathrm{s}\right.$ ]

$\rho \quad$ Density

$\left[\mathrm{kg} / \mathrm{m}^{3}\right]$

$\sigma_{r} \quad$ Reduced stress

[Pa]

$\tau \quad$ Time delay

[s]

$\tau \quad$ Viscous stress tensor

$\left[\mathrm{kg} /\left(\mathrm{m}^{2} \mathrm{~s}\right)\right]$

$\tau_{w} \quad$ Wall shear stress

[Pa]

$\omega \quad$ Angular frequency

$[\mathrm{rad} / \mathrm{s}]$ 


\section{AbBreviations}

$A E \quad$ Acousto-elastic analysis

$B S \quad$ Beam splitter

$C C D \quad$ Camera

CFD Computational Fluid Dynamics

CFL Courant-Friedrichs-Lewy number

DNS Direct Numerical Simulation

ED/FRC Eddy Dissipation/Finite Rate Chemistry combustion model

FEA Finite Element Analysis

FEM Finite Element Method

FFT Fast Fourier Transform

FSI Fluid-structure interaction

FTF Flame Transfer Function

LES Large Eddy Simulation

$L D V \quad$ Laser Doppler Vibrometer

PLIF Planar Laser Induced Fluorescence

RANS Reynolds Average Navier-Stokes

RMS Root Mean Square

SAS Scale Adaptive Simulation

SPL $\quad$ Sound Pressure Level

URANS Unsteady Reynolds Average Navier-Stokes

\section{SUPERSCRIPTS AND SUBSCRIPTS}

$\begin{array}{cl}0 & \text { Mean value } \\ 1,2,3 & \text { Components } \\ +,- & \text { Directions }\end{array}$




$\begin{array}{cl}\cdot & \text { Perturbations } \\ - & \text { Mean value } \\ a & \text { Transpose } \\ a & \text { Air } \\ f & \text { Fuel } \\ f & \text { Fluid } \\ i, j, k, l, m, n & \text { Components } \\ p & \text { Plate } \\ s & \text { Structure } \\ s t & \text { Stoichiometric value } \\ w & \text { Wall } \\ x, y, z & \text { Directions }\end{array}$





\section{APPENDiX A}

\section{Numerical and Analytical Model of a Plate}

To validate results obtained during numerical calculations of combustion chamber geometry, a model of a plate is investigated first. The plate is supported on four sides by clamped boundary conditions as presented in Figure 1. The width/length ratio of the plate is equal to 0.4 which corresponds to data given for $\lambda^{2}{ }_{\text {ij }}$ by (Blevins R. , 1984), see Table 1 . Natural frequencies of the plate are calculated according to Equation 1. Material properties of stainless steel SS 310 are presented in Table 2. For numerical model, the Shell 63 elements equally distributed over the plate are employed. Isentropic material properties are assumed.

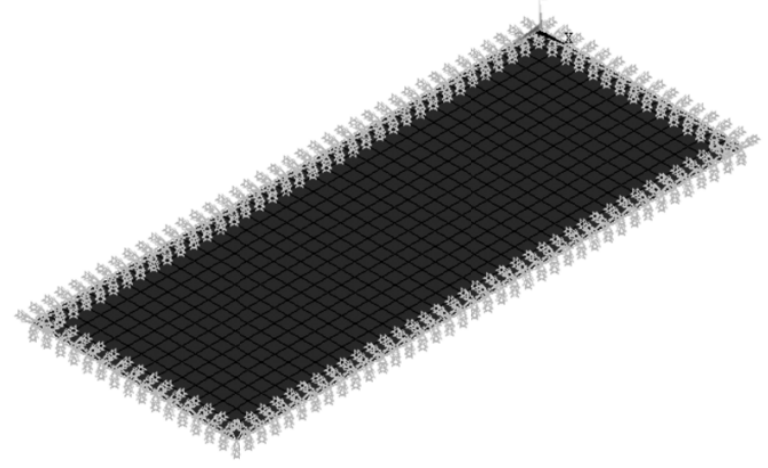

Figure 1: Model of the plate 


$$
f_{i j}=\frac{\lambda_{i j}^{2}}{2 \pi a^{2}} \sqrt{\frac{E h^{3}}{12 \gamma\left(1-v^{2}\right)}}
$$

\begin{tabular}{cc} 
Mode & $\lambda_{i j}{ }^{2}$ \\
\hline 1 & 23.65 \\
2 & 27.82 \\
3 & 35.45 \\
4 & 46.70
\end{tabular}

Table 1: $\lambda_{\mathrm{ij}}{ }^{2}$ value for plate with clamped boundary conditions

\begin{tabular}{ccc} 
Symbol & Description & Value \\
\hline$E$ & Young's modulus & $210[\mathrm{GPa}]$ \\
$h$ & Plate thickness & $0.001[\mathrm{~m}]$ \\
$a$ & Plate width & $0.15[\mathrm{~m}]$ \\
$\gamma$ & Mass per unit area & $7.8\left[\mathrm{~kg} / \mathrm{m}^{2}\right]$ \\
$v$ & Poisson's ratio & $0.3[-]$ \\
$\rho$ & Density & $7800\left[\mathrm{~kg} / \mathrm{m}^{3}\right]$
\end{tabular}

Table 2: Material properties and geometrical dimensions of the plate

Numerical and analytical results are in good agreement, see Table 3. Based on this fact, it is assumed that the eigenfrequency results obtained from numerical computations of the liner entire geometry will match well the real system behaviour.

Analytical calculations

$\begin{array}{ll}262.68 & 262.30 \\ 308.99 & 308.19 \\ 393.74 & 392.18 \\ 518.69 & 516.49\end{array}$

Table 3: Analytical and numerical results of plate eigenfrequencies 


\section{APPENDIX B}

\section{NATURAL FREQUENCIES OF THE LINER}

To investigate fluid-structure interaction the liner with maximized flexibility and well controlled acoustic and structural boundary conditions is needed. To fulfil these requirements several liners with different shape, thickness and length of flexible section are considered, see Table 1. The influence of the combustion chamber and cooling passage cavities at elevated temperature is tested as well. Computations are done with the use of Ansys code based on the finite element method. For a geometry discretization, a uniform distributed mesh of Shell 63 (structure) and Fluid 30 (fluid - fluid and fluid - structure) element types is used. Properties of the stainless steel and air are taken at room temperature $\left(20^{\circ} \mathrm{C}\right)$ and at elevated combustion temperature (temperature of hot gases and steel equal to $1500^{\circ} \mathrm{C}$ and $760^{\circ} \mathrm{C}$, respectively). Pressure degrees of freedom from the combustion chamber and from the cooling passage are coupled directly to the structural nodes. To prevent a direct nodes connection from the air cavities, structural part is divided into two connected together segments. Both, inlet and outlet of air cavities is modelled as acoustically hard walls. As dynamic liner behaviour depends substantially on flexible part, see Figure 1 only this section is modelled in the Ansys code.

\begin{tabular}{ccc} 
Flexible liner & Rectangular & Square \\
\hline Cross-section $[\mathrm{mm} \times \mathrm{mm}]$ & $150 \times 50$ & $150 \times 150$ \\
Thickness $[\mathrm{mm}]$ & $0.8-1.2$ & $0.8-1.2$ \\
Length $[\mathrm{mm}]$ & $200-680$ & $200-680$
\end{tabular}

Table 1: Models geometry and dimension 


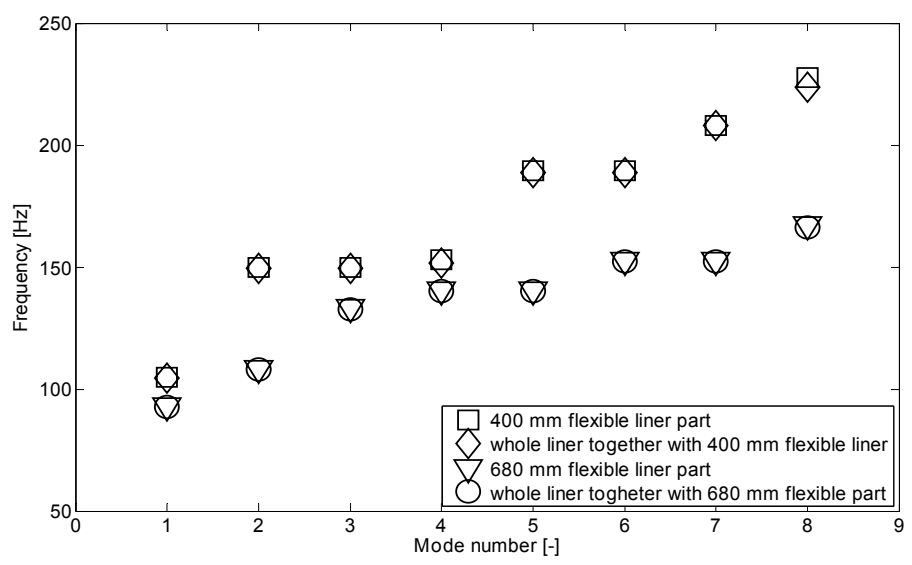

Figure 1: Eigenfrequency comparison of the flexible section of the liner only and entire liner (for two lengths)

The stiffness of the liner plays important role in fluid-structure interaction. Comparison of square and rectangular cross-section liner reveals that the rectangular liner with $0.8 \mathrm{~mm}$ thickness and length of $680 \mathrm{~mm}$ is less flexible than the square liner with the same dimensions, see Figure 2. The first eigenfrequency observed in case of the rectangular shaped liner is located at $142 \mathrm{~Hz}$, whereas for the square liner configuration at approximately $92 \mathrm{~Hz}$. Therefore, further analysis is restricted to the liner with square crosssection.

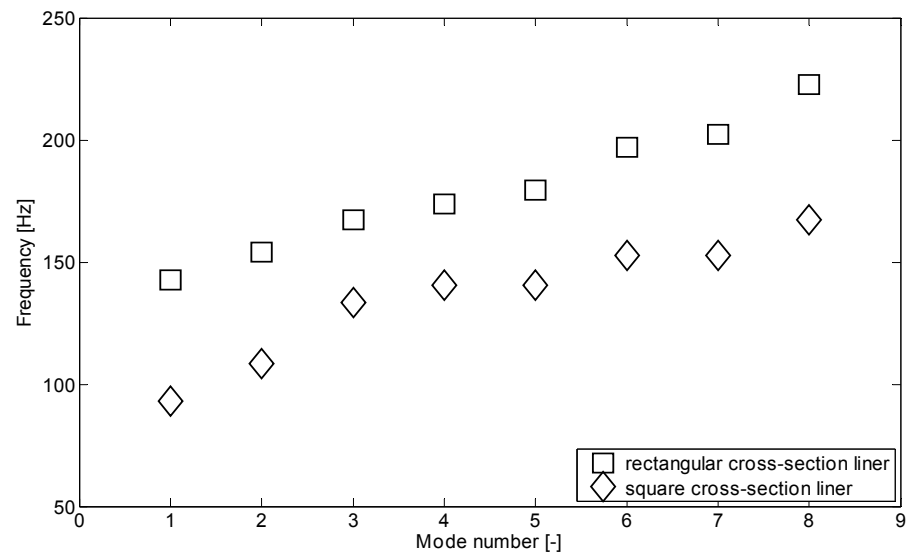

Figure 2: Eigenfrequency of liner with rectangular and square cross-section area (same length and thickness of the flexible part) 
The flexibility of the liner depends strongly on the flexible part thickness, as it is shown in Figure 3. Decreasing the wall thickness lowers the eigenfrequencies. However, it is not possible to decrease the wall thickness substantially due to significant negative effect on the liner strength and risk of the test rig damage. Additionally, problems with welding parts of the liner with very small thickness might appear. Therefore, it is assumed that the liner thickness of $0.8 \mathrm{~mm}$ is the lowest which assure safe operating conditions.

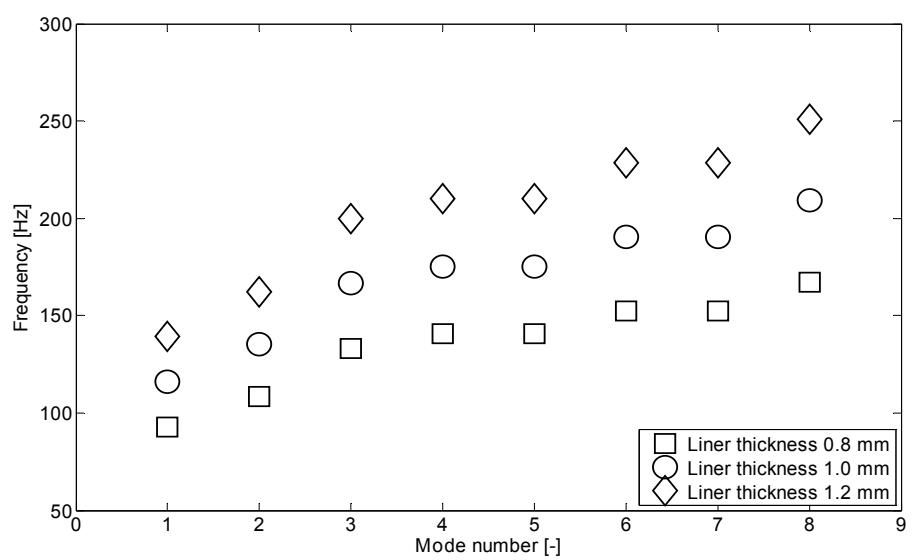

Figure 3: Liner thickness influence on the eigenfrequencies (square cross-section liner with constant walls length)

The investigation of the length influence on the eigenfrequencies is limited mainly by thermocouples and pressure transducers connections located in the vicinity of the flexible liner. To prevent vibration damping due to the connections of measurement devices to a flexible section, all thermocouples, pressure transducers etc. are located in some distance from vibrating wall. Since the position of these devices is already fixed, the maximal possible length of the thin liner is $680 \mathrm{~mm}$.

The eigenfrequencies pattern show significant difference in results between $200 \mathrm{~mm}$ and $400 \mathrm{~mm}$ length of the liner, see Figure 4. Increasing the length of the liner above $400 \mathrm{~mm}$ decreases the eigenfrequency of the walls but the effect is less significant. Since the flexibility is a very important issue for the fluid structure interaction the maximum possible liner with length equal to $680 \mathrm{~mm}$ is chosen for further research. 


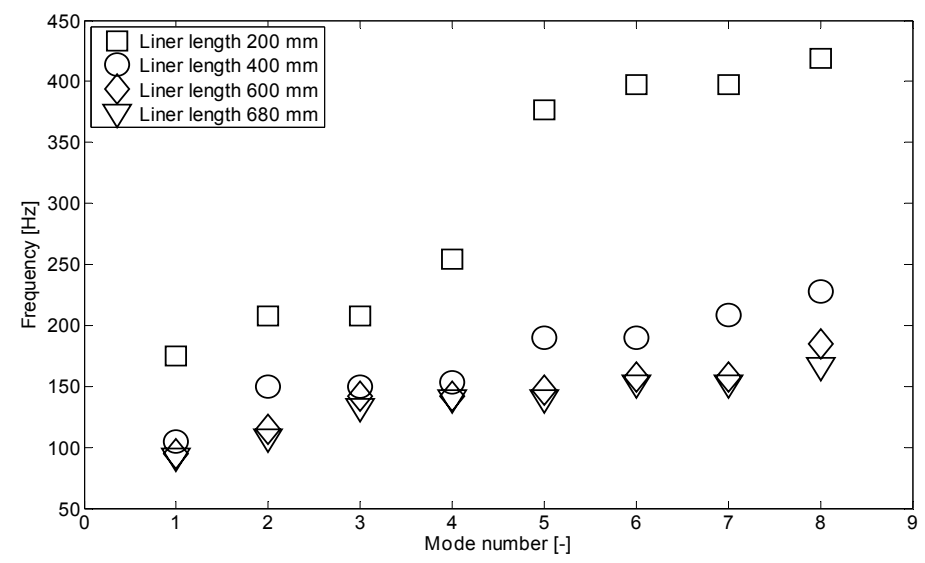

Figure 4: Comparison of eigenfrequencies for different liner lengths

The air located in the combustion chamber and cooling passage cavities due to different density might have also influence on the vibration patter. To predict the influence of air, the model of combustion chamber and cooling passage cavities coupled with wall structure is designed. More about the model can be found in Chapter 5.

The results presented in Figure 5 show a minor influence of the cavities on the vibration eigenfrequencies of the liner. The effect of air gaps due to small mass of air is minor with comparison to geometrical influence.

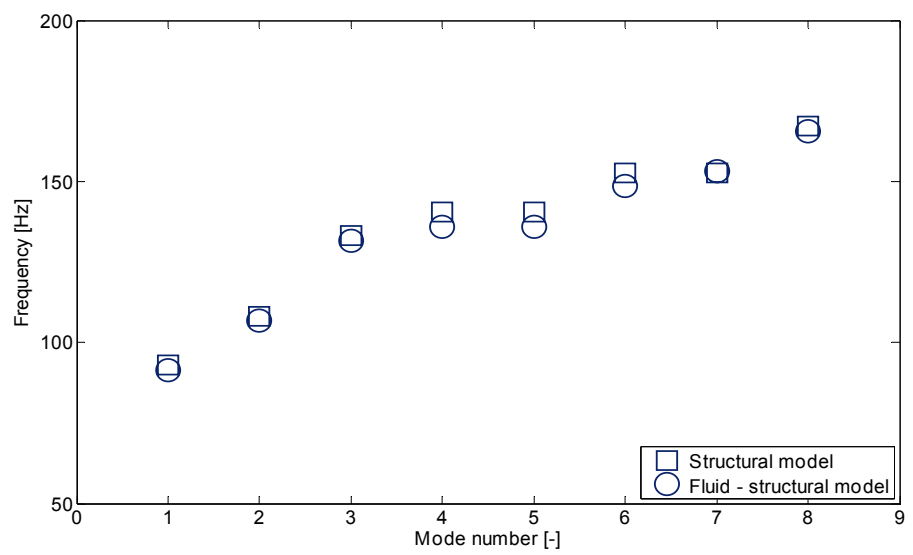

Figure 5: Eigenfrequency of structural and fluid-structural model 
All the aforementioned investigations are performed at room temperature. Because the combustion test rig operates at elevated temperature it is necessary to include it in the eigenfrequency prediction. Combustible gas inside the combustion chamber is not any more air only but a mixture of air, fuel and product gases. Due to excess of air during combustion process (lean combustion), it is assumed that inside both: combustion chamber and cooling there is air with temperature of $1500^{\circ} \mathrm{C}$ and $150^{\circ} \mathrm{C}$, respectively. Material properties of stainless steel SS 310 are equal to the properties at temperature of $760^{\circ} \mathrm{C}$. Calculations are done for two models: liner structural part only and liner together with combustion chamber and cooling passage.

A substantial decreasing of eigenfrequencies due to elevated temperature can be observed, see Figure 6 and Figure 7. This behaviour is mainly the consequence of changes in material properties of steel. Introduction of hot air inside the combustion chamber and cooling passage again did not affect the eigenfrequencies.

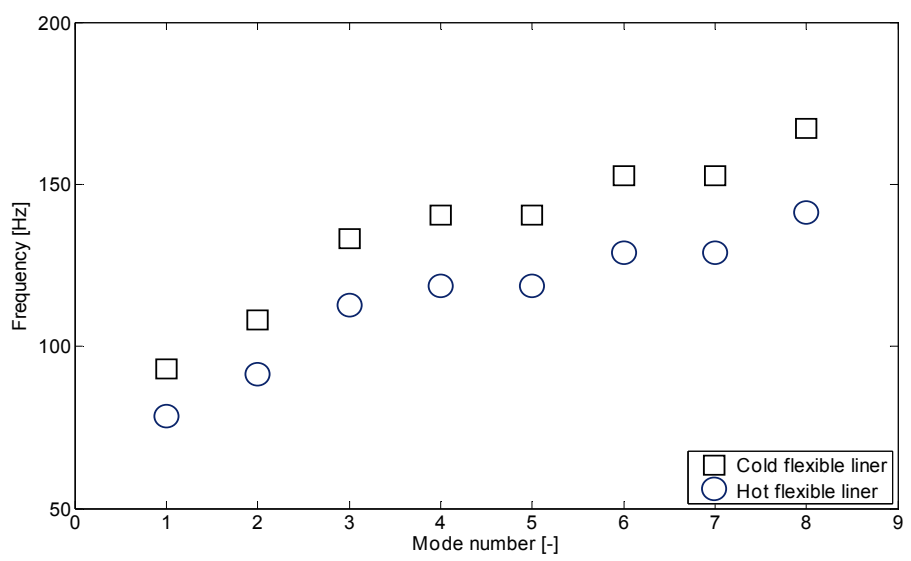

Figure 6: Temperature influence on eigenmodes - structural models

The results obtained during numerical investigation show that significant decrease of the liner eigenfrequencies can be obtained by the modification of liner shape or geometrical walls dimensions. Observed system behaviour is caused by variations in second moment of area and mass of solid body. Other source of diminishing eigenfrequencies is an elevated temperature which changes substantially material properties e.g. Young's modulus is lower about $30 \%$ in case of hot liner work conditions with comparison to room temperature. Influence of surrounding air cavities is not as significant as geometrical dimensions and material properties but it also affects eigenfrequencies. Vibrating body induces vibrations 
of the air in combustion chamber and cooling passage, which are source of additional inertia forces due to mass of air. The computations show that the most flexible liner is one with the square cross-section with $0.8 \mathrm{~mm}$ thickness and $680 \mathrm{~mm}$ length of the flexible part. However, due to the technical difficulties with welding thin parts of steel sheets, the liner with thickness of $1 \mathrm{~mm}$ is chosen.

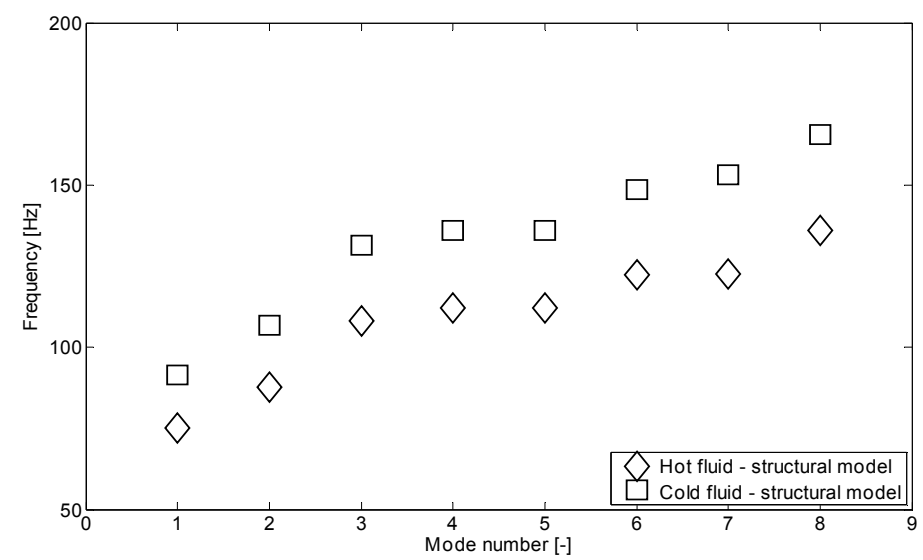

Figure 7: Temperature influence on eigenmodes - fluid-structural models

Solutions obtained from the Ansys analysis shows 'one dimensional' pattern of the eigenmodes, as shown on Figure 8. This is influence of small width/length ratio and also consequence of the clamped boundary conditions of the liner model.

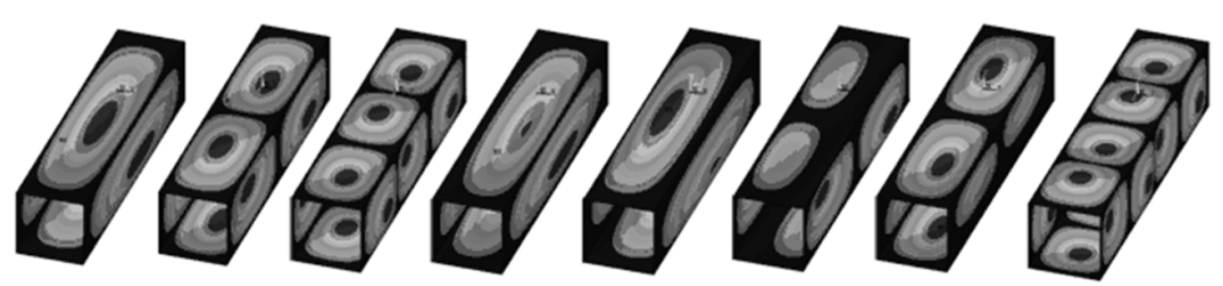

Figure 8: First $8^{\text {th }}$ eigenmodes of the flexible liner with length of $680 \mathrm{~mm}$ 


\section{APPENDIX C}

\section{STRESS AND DisPlaCEMENT ANALYSIS}

In order to verify strength of the liner (because of lower thickness Fluistcom liner configuration is more sensitive for high amplitude pressure oscillations) and to assure safety work conditions, a numerical investigation of stress and deformation pattern is done using FEM code - Ansys. The liner model is investigated at $760{ }^{\circ} \mathrm{C}$ which is a typical temperature of walls during the combustion process. Since the liner is split into two parts (top and bottom) by sliding connection to prevent mutual interaction between them, only the part of the combustion chamber with the flexible section is under research. The bottom edges of this part are clamped, whereas the top one can expand. This configuration resembles the experimental liner where a bottom section is restricted by flange and top one can expands inside the sliding connection, see Chapter 2. The numerical results are evaluated according to von Misses theory where a reduced stress $\left(\sigma_{\mathrm{r}}\right)$ is calculated as combination of principal stresses $\left(\sigma_{1}, \sigma_{2}, \sigma_{3}\right)$, see Equation 1 .

$$
\sigma_{r}=\sqrt{\frac{\left(\sigma_{1}-\sigma_{2}\right)^{2}+\left(\sigma_{2}-\sigma_{3}\right)^{2}+\left(\sigma_{3}-\sigma_{1}\right)^{2}}{2}}
$$

The results show maximal reduced stress in the range of several kPa mainly at the location of sharp edges of the liner. This stress is slightly overestimated due to rough corners of the walls (typically corners are rounded to reduce unphysical accumulation of stress at some nodes). Even though, the calculated stress is much below the plasticity limit of stainless steel 310 , which is approximately at about $160 \mathrm{MPa}$. Thermal expansion of the walls in longitudinal direction computed by Ansys due to heat transfer is predicted as $15.12 \mathrm{~mm}$, 
which means that the liner can expand freely without exerting additional stresses on the top part of the chamber. Therefore the thermal stresses which appear in material have minor effect on the liner performance and they are not investigated further.

To investigate the influence of pressure difference between combustion chamber and cooling passage on walls vibration it is assumed that the liner has uniform temperature of $760{ }^{\circ} \mathrm{C}$ and material properties of stainless steel adequate to this temperature. Furthermore, a pressure difference between both cavities equal to $5 \mathrm{kPa}$ is imposed on the liner walls. This is the maximal pressure difference at which the test rigs should stay in a safety mode; and it is about two to three times bigger than commonly observed pressure fluctuations during combustion experiment.

The maximal reduced stress obtained during calculations is located on the sharp edges of the liner model, see Figure 1. The highest observed value of stress is approximately 45 $\mathrm{MPa}$, which is far below the yield point for this kind of stainless steel. Static walls displacement equal to around $0.3 \mathrm{~mm}$ is recorded. Since the conducted research involves only steady analysis, the maximal displacement is observed in the middle of flexible liner section. However, exceeding the limit of pressure difference $(5 \mathrm{kPa})$ between combustion and cooling passage is not advisable. Under transient working conditions rapid changes in the pressure and stress field might occur. They can lead first to liner plastic deformation and latter to the system failure.
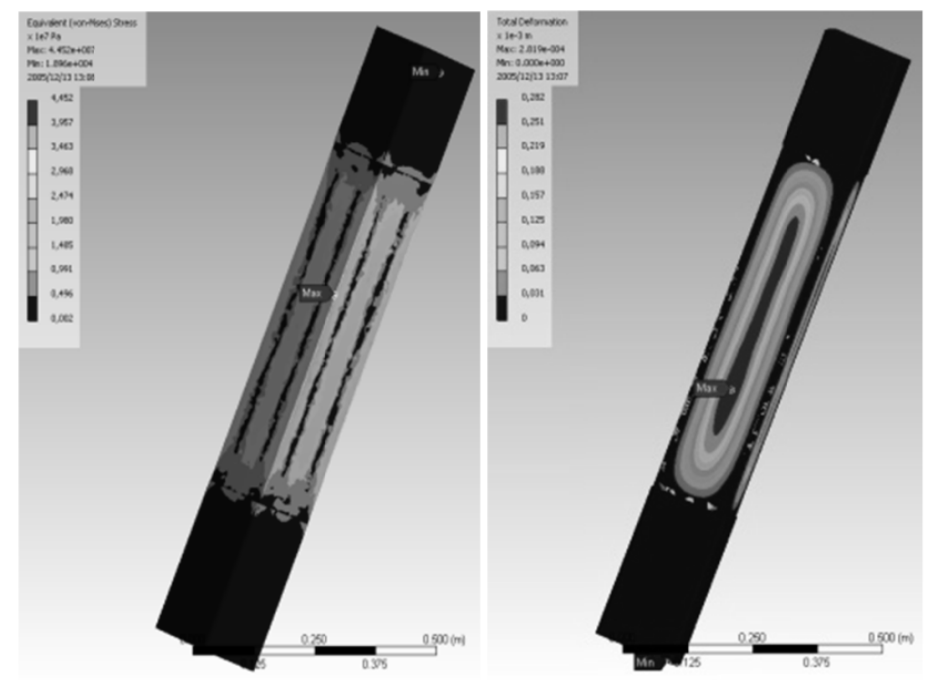

Figure 1: Stress (left) and displacement (right) pattern obtained during loads analysis 


\section{APPENDIX D}

\section{EXPERIMENT With Instabilities AT First ACOUSTIC Mode}

Next to the typically visible instabilities at around $440 \mathrm{~Hz}$ for Desire liner configuration, during one of the combustion experiment, instabilities at frequency of 247 $\mathrm{Hz}$ were observed, see Figure 1. They were recorded for both steady and pulsating flow conditions.

The behaviour of the system was unusual, since at that frequency the thermo-acoustic instabilities were never before visible. However, the experimental results were in good agreement with prediction of thermo-acoustic instabilities by (Van Kampen, 2006). According to his model, the instabilities might occur near first or second acoustic eigenfrequency of the combustion chamber. The results presented here show strong peak around the first acoustic eigenfrequency of the system. The secondary instabilities are located approximately at third and fourth acoustic mode. Only small disturbance in the signal is noticeable at second system eigenfrequency, thus the main instabilities were shifted entirely to lower frequency region. The overall pressure level in the combustion chamber is similar to the case with instabilities at $440 \mathrm{~Hz}$. The differences are only visible for pressure transducer P2 and velocity signal. Here, the SPL is significant low for the instabilities at $247 \mathrm{~Hz}$. Vibrations of the liner are also only a fraction of the vibrations during the other investigated case. This could be an effect of different vibration mode (point of measurements is located near the vibration node). The reason of instabilities appearing on the first eigenfrequency mode is not know. 

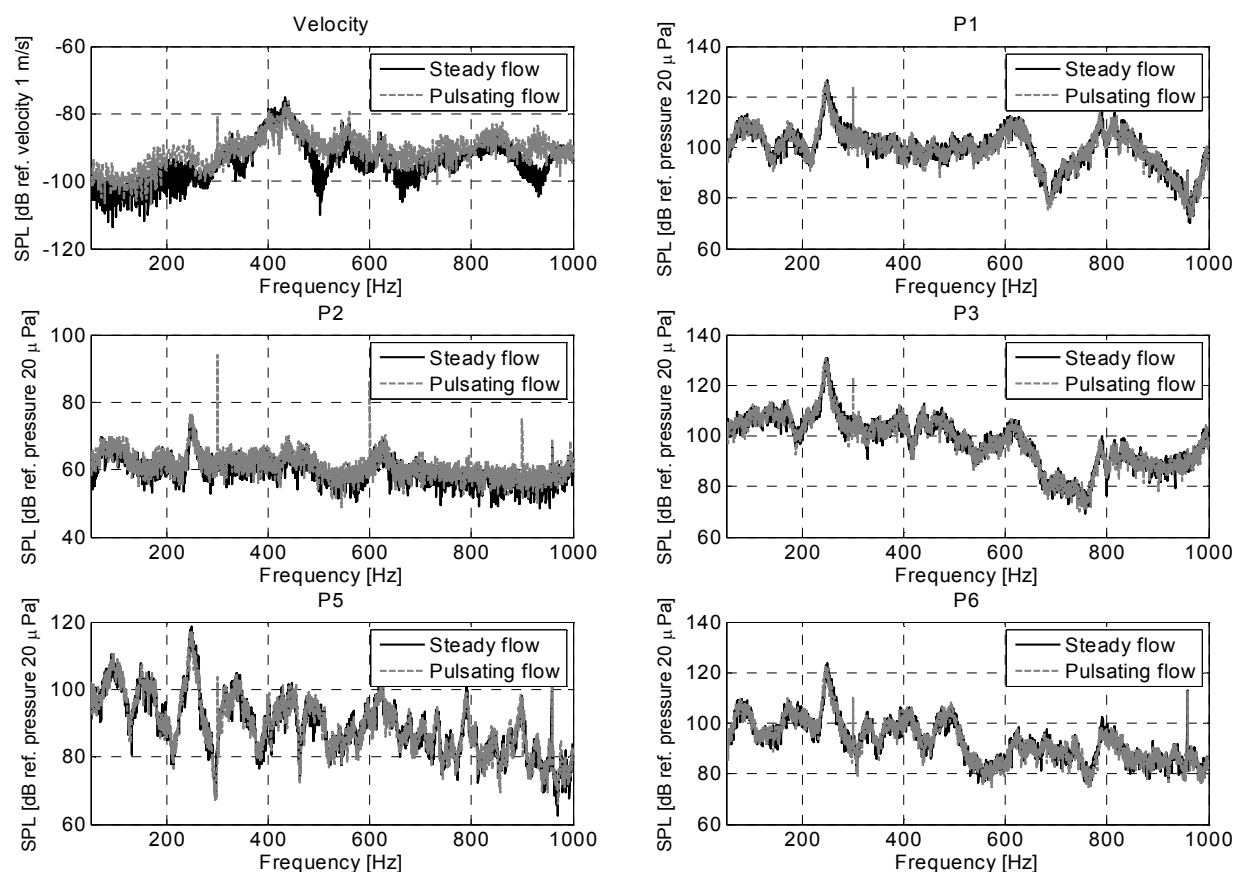

Figure 1: Combustion instabilities of the Desire liner configuration visible at around first acoustic eigenfrequency of the combustion chamber 


\section{APPENDIX E}

\section{CFI MODEL}

Next to standard available in the Ansys CFX code combustion models, a housemade CFI combustion model is employed for the combustion calculations. Using chemical models from the commercial codes with a very simplified chemistry can give quick and good solution, however those models do not take into account the interaction between various components of the fuel as well as an effect of turbulence on the chemistry. In order to study their effect on the combustion the CFI model based on the FIRST model by (Kok \& Louis, 1998) was developed by (Derksen, 2005). A detailed chemical mechanism GRI 3.0 which involves 325 reactions and 53 species is used for the combustion process modelling. However, solving all the necessary transport equations for the species needs a significant, additional computational effort with comparison to standard commercial combustion models. Therefore, in order to reduce this effort, the reaction progress variables (RPV), based on the CSP algorithm, see (Lam \& Goussis, 1994), is used. The RPV describes the detailed chemistry mechanism. In this process the fast reactions which occur during combustion are assumed to have a minor contribution to the overall process chemistry and they are removed from the mechanism. This, reduce the computational effort with simultaneously good approximation of the resolved chemical reaction rate. The CFI and FIRST models developed at the Laboratory of Thermal Engineering were successfully used for simulation of combustion of different types of fuels, see (Kok \& Louis, 1998), (Louis, 1997), (Derksen, 2005), (Van Kampen, 2006), (Pater, 2007), (De Jager, 2007). Detailed information about the CFI model can be found in the abovementioned references.

In the present study the comparison is made between a standard CFX-Ansys 11, ED/FRC combustion model and the CFI house made code. Since the main focus is the mutual interaction between acoustics, combustion and vibrations, the influence of the combustion model on the flame resolving is the main priority here. It can be seen in Figure 1 that both models predicted a maximum adiabatic temperature of the combustion process around 1800 
$\mathrm{K}$. They captured similarly the flame shape, flame opening angle and location where the flame is anchored. The velocity field and recirculation zones have shown also alike magnitude and shape. The only visible difference was the flame length which in case of the CFI combustion model is slightly shorter. Surprisingly, the commercial ED/FRC code predicted the length of the flame with higher accuracy than the CFI model. The reason is the CFI model originally was developed for the diffusion flames. Because of that, it predicts better the diffusion, stoichiometric and rich turbulent combustion. Here, the lower temperature occurring during a lean premixed combustion leads to an over-prediction of the source term (especially in the lean regime) and consequently to decrease in the flame length. It was possible to adjust this difference however, the manual interference in to the source term was necessary. Furthermore, the total time of implementation and computation of the CFI model was few times longer than ED/FRC model. On the other hand the $\mathrm{ED} / \mathrm{FRC}$ model has suffered from a lack of the possibility to calculate some of the species spectrum (e.g. $\mathrm{OH}, \mathrm{CH}$ etc.) involved in the process.

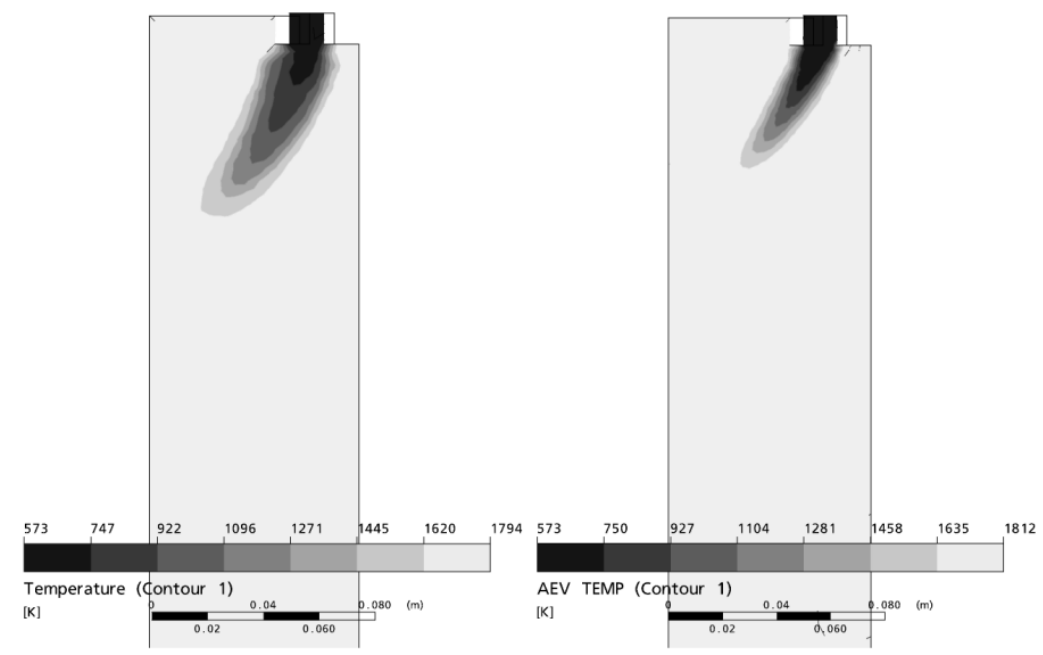

Figure 1: Comparison of the temperature field between the CFX-Ansys ED/FRC combustion model (left) and CFI combustion model (right)

Taking into account all the aforementioned benefits and drawbacks of both combustion models, and having in mind that for a prediction of thermo-acoustic instabilities the length of the flame and computational time are important issues, the ED/FRC combustion model was chosen for further multi-field interaction. 


\section{BIBLIOGRAPHY}

1. ADINA R\&D, I. (2008). Retrieved from http://www.adina.com/adinafsi.shtml

2. Ansys. (2007). HTML Online Documentation. Ansys Inc.: Ansys 11.

3. Baker, A. J. (1983). Finite Element Computational Fluid Mechanics. Washington: Hemisphere Publishing Corporation.

4. Bellucc, V., Rohr, P., Paschereit, C., \& Magni, F. (2004). On the use of Helmholtz resonators for damping acoustic pulsations in industrial gas turbines. J. Eng. Gas Turbines Power, 126, 271-275.

5. Belov, A. V., Kaplunov, J. D., \& Nolde, E. V. (1999). A Refined Asymptotic Model of Fluid-Structure Interaction in Scattering by Elastic Shells. Flow, Turbulence and Combustion , 61, 255-267.

6. Bird, R., Stewart, W., \& Lightfoot, E. (2002). Transport Phenomena, Second Edition. John Wiley \& Sons, Inc.

7. Blevins, R. D. (1984). Formulas for natural frequency and mode shape. New York: Robert E. Krieger publishing company.

8. Blom, C. (2003). Discontinuous Galerkin Method on Tetrahedral Elements for Aeroacoustics. Enschede: $\mathrm{PhD}$ thesis, University of Twente.

9. Blom, F. J. (1998). A monolithical fluid-structure interaction algorithm applied to the piston problem. Computer methods in applied mechanics and engineering , 167, 369391.

10. Breard, C., Sayma, A., Vahdati, M., \& Imregun, M. (2002). Aeroelasticity analysis of an industrial gas turbine combustor using a simplified combustion model. Journal of Fluids and Structures , 16(8), 1111-1126. 
11. Cabot, G., Vauchelles, D., Taupin, B., \& Boukhalfa, A. (2004). Experimental study of lean premixed turbulent combustion in a scale gas turbine chamber. Experimental Thermal and Fluid Science, 28, 683-690.

12. Chu, B. (1964). On the energy transfer to small disturbances in fluid flow (Part 1). Acta Mechanica , 1 (3), 215-234.

13. CFX Ansys 11. (2007). HTML Online Documentation.

14. CFX-10. (2005). Retrieved from CFX 10, Online Documentation.

15. Cho, J., \& Lieuwen, T. (2005). Laminar premixed flame response to equivalence ratio oscillations. Combustion and Flame, 140, 116-129.

16. Cohen, H., Rogers, G., \& Saravanamuttoo, H. (1996). Gas Turbine Theory (4th edition ed.). London: Longman Group Limited.

17. Crighton, D., Dowling, A., Ffowcs Williams, J., Heckl, M., \& Leppington, F. (1992). Modern Methods in Analytical Acoustics. London: Springer-Verlag.

18. Davis, R. (2008). Techniques to assess acoustic-structure interaction in liquid rocket engines. Durham: PhD thesis, Duke University.

19. De Jager, B. (2007). Combustion and noise phenomana in turbulent alkane flames. Enschede: PhD thesis, University of Twente.

20. Derksen, M. A. (2005). On the influence of steam on combustion. Enschede: $\mathrm{PhD}$ thesis, University of Twente.

21. Dowling, A., \& Morgans, A. (2005). Feedback control of acoustic instabilities. Annu. Rev. Fluid Mech. , 37, 151-182.

22. DSP-SigLab. (2001). User Guide. Spectral Dynamic Inc.

23. Ducruix, S., Schuller, T., Durox, D., \& Candel, S. (2003). Combustion Dynamics and Instabilities: Elementary Coupling and Driving Mechanisms. Journal of Propulsion and Power , 19, 722-734.

24. Engel, Z. (2001). Ochrona srodowiska przed drganiami i halasem. Warsaw: Wydawnictwo Naukowe PWN.

25. Farath, C., Lesoinne, M., \& Le Tallec, P. (1998). Load and Motion transfer Algorithms for Fluid/Structure Interaction Problems with Non-Matching Descrete Interfaces: Momentum and Energy Conservation, Optimal Discretization and Application to Aeroelasticity. Computer Methods in Applied Mechanics and Engineering , 157, 95-114. 
26. Felippa, C. A. (2004). ASEN 5519-006: Fluid-Structure Interaction. Retrieved from http://www.colorado.edu/engineering/CAS/courses.d/FSI.d/Home.html

27. Felippa, C. A., Park, K. C., \& Farhat, C. (2001). Partitioned analysis of coupled mechanical systems. Computer Methods in Applied Mechanics and Engineering , 190, 3247-3270.

28. FLECS. (2008). Retrieved from http://www.aero.lr.tudelft.nl/FLECS

29. Forsythe, N. (2006). A partitioned approach to fluid-structure interaction for artificial heart valves. Belfast: $\mathrm{PhD}$ thesis, Queen's University Belfast.

30. Gasparetti, M., Paone, N., \& Tomasini, E. (1996). Laser Doppler techniques for the combined measurement of inlet flow and valve motion in IC engines. Meas. Sci. Technol. , 7, 576-591.

31. Golliard, J. (2002). Noise of Helmholtz-resonator like cavities excited by a low Machnumber turbulent flow. Poitiers: PhD thesis, University of Poitiers.

32. Guerri, O., Hamdouni, A., \& Sakout, A. (2008). Numerical simulation of the flow around oscillating wind turbine airfoils, Part 1: Forced oscillating airfoil. International Journal of Multiphysics , 2 (4), 367-385.

33. Hameyer, K., Driesen, J., De Gersem, H., \& Belmans, R. (1999). The Classification of Coupled Field Problems. IEEE Transactions on Magnetics , 35 (3), 1618-1621.

34. Hannink, M. (2007). Acoustic resonators for the reduction of sound radiation and transmission. Enschede: PhD thesis, University of Twente.

35. Harleman, M. (2005). Characterizing flame behaviour in a lean premixed turbulent methan burner with optical techniques. Enschede: MSc thesis, University of Twente.

36. Heckl, M. (1988). Active control of noise form a Rijke tube. Journal of Sound and Vibration , 124, 117-133.

37. Hermann, M. G., \& Steindorf, J. (2003). Partitioned strong coupling algorithms for fluid-structure interaction. Computers and Structures , 81, 805-812.

38. Hermann, M. G., \& Steindorf, J. (2002). Strong Coupling Methods. Stuttgart Multifield Conference. Stuttgart.

39. Hirschberg, A. (2004). Introduction to aero-acoustics of internal flows. Enschede: Course materials.

40. Hobson, D., Fackrell, J., \& Hewitt, G. (2000). Combustion Instabilities in Industrial Gas Turbines - Measurements on Operating Plant and Thermoacoustic Modelling. ASME , 122, 420-428. 
41. Hron, J., \& Turek, S. (2006). A monolithic FEM/Multigrid solver for ALE formulation of fluid structure interaction with application in biomechanics. Ergebnisberichte des Instituts für Angewandte Mathematik, 311.

42. Hubbard, S., \& Dowling, A. (2001). Acoustic Resonances of an Industrial Gas Turbine Combustion System. Journal of Engineering for Gas Turbines and Power, 123, 766-773.

43. Hubner, B., \& Seidel, U. (2007). Partitioned solution to strongly coupled hydroelastic systems arising in hydro turbine design. 2th IAHR Internaitonal Meeting of the Workgoup on Cavitation and Dynamic Problems in Hydraulic Machinery and Systems, (pp. 55-64). Timisoara.

44. Huls, R. (2006). Acousto-Elastic Interaction in Combustion Chambers. Enschede: $\mathrm{PhD}$ thesis.

45. Jarosinski, J. (1996). Techniki czystego spalania. Warsow: Wydawnictwo NaukowoTechniczne.

46. Jovic, S., \& Driver, D. (1994). Backward-facing step measurement at low Reynolds number, Reh=5000. NASA Tech. Mem. , 108807.

47. Jovic, S., \& Driver, D. (1995). Reynolds number effect on the skin friction in separated flows behind a backward-facing step. Exps. Fluids , 18, 464-467.

48. Kaczor, A., \& Sygulski, R. (2005). Free vibration analysis of floating plates. 6th International Conference 'ENVIRONMENTAL ENGINEERING', (pp. 774-777). Vilnius.

49. Khatir, Z., Pozarlik, A., Cooper, R., Watterson, J., \& Kok, J. (2006). Numerical study of coupled fluid-structure interaction for combustion system. Proceedings of Conference on Numerical Methods for Fluid Dynamics. Reading.

50. Klein, S. (2000). On the acoustics of turbulent non-premixed flames. Enschede: $\mathrm{PhD}$ thesis, University of Twente.

51. Kleinlugtenbelt, J. (2005). Acoustic flow oscillations in fuel supply lines. Enschede: Master thesis, University of Twente.

52. Kok, J., \& Louis, J. (1998). Modeling turbulent combustion in CO/H2 diffusion flame using reaction progress variables. Combust. Sci. and Tech. , 131, 225-249.

53. Laherrere, J. (2006). Fossil fuels, what future? Workshop Global Dialogue on Energy Security. Beijing. 
54. Lam, S., \& Goussis, D. (1994). The CSP method for simplifying kinetics. International Journal of Chemical Kinetics , 26, 461-486.

55. Launder, B. E., \& Spalding, D. B. (1974). The numerical computation of turbulent flows. Computer Methods in Applied Mechanics and Engineering , 3, 269-289.

56. Le, H., Moin, P., \& Kim, J. (1997). Direct numerical simulation of trubulent flow over a backward-facing step. J. Fluid Mech. , 330, 349-374.

57. Lefebvre, A. (1983). Gas turbine combustion. Hemisphere Publishing Corporation.

58. Lieuwen, T. (2003). Combustion Driven Oscillations in Gas Turbines. Turbomachinery International, 44, 16-18.

59. Lieuwen, T., Torres, H., Johnson, C., \& Zinn, B. (2001). A Mechanism of Combustion Instability in Lean Premixed Gas Turbine Combustors. Journal of Engineering for Gas Turbines and Power, 123, 182-189.

60. Lighthill, M. J. (1952). On Sound Generated Aerodynamically, I. General Theory. Porceedings of the Royal Society of London, Series A, Mathematical and Physical Sciences , 211, 564-587.

61. Lighthill, M. J. (1954). On Sound Generated Aerodynamically, II. Turbulence as a Source of Sound. Proceedings of the Royal Society of London, Series A. Mathematical and Physcal Sciences, 222, 1-31.

62. Linne, M. A. (2002). Spectroscopic Measurement. An Introduction to the Fundamentals. London: Academic Press.

63. Louis, J. (1997). On turbulent combustion of coal gas. Enschede: University of Twente, PhD thesis.

64. Matweb. (2008). Retrieved from http://www.matweb.com

65. Menter, F. R., Kuntz, M., \& Bender, R. (2003). A scale-adaptive simulation model for turbulent flow prediction. AIAA.

66. Menter, F., \& Egorov, Y. (2005). Scale-Adaptive Simulation fuer Technische Stroemungen. Stuttgart: Ansys Germany.

67. Mitchell, A. R. (1980). The finite difference method in partial differential equations. Chichester: Wiley.

68. MPCCI. (2008). Retrieved from http://www.mpcci.de/

69. National Instrument, C. (2003). LabVIEW User Manual. Austin: National Instrument Corporate. 
70. Nicoud, F., \& Poinsot, T. (2005). Thermoacoustic instabilities: Should the Rayleigh criterion be extended to include entropy changes. Combustion and Flame, 142(1), 153-159.

71. OASIS4. (2008). Retrieved from http://www.prism.enes.org/PAEs/coupling_IO/software_OASIS4.php

72. Oskam, A. (1999). Flow and Heat Transfer in Residential Heating Systems. Enschede, The Netherlands: Master Thsesis, University of Twente.

73. Paone, N., \& Revel, G. M. (2000). Laser vibration measurements through combustive flows: application to an industrial burner in working conditions. Measurement , 28, 21-31.

74. Paschereit, C., Schuermans, B., Polifke, W., \& Mattson, O. (2002). Measurement of Transfer Matrices and Source Terms of Premixed Flames. Journal of Engineering for Gas Turbines and Power , 124, 239-247.

75. Pater, S. G. (2007). Acoustics of turbulent non-premixed syngas combustion. Enschede: PhD thesis, University of Twente.

76. Penrose, J. M., \& Staples, C. J. (2002). Implicit fluid-structure coupling for simulation of cardiovascular problems. International Journal for Numerical Methods in Fluids, 40, 467-478.

77. Poinsot, T., \& Veynante, D. (2005). Theoretical and Numerical Combustion. Second Edition. Philadelphia: Edwards.

78. Polifke, W., Poncet, A., Paschereit, C., \& Doebbeling, K. (2001). Reconstruction of acoustic transfer matrices by instationary computational fluid dynamics. Journal of Sound and Vibration, 245(3), 483-510.

79. PolytecGmbH. (2003). Measurement Solutions Made Possible by Laser Vibrometry. Polytec LM INFO Special .

80. Pozarlik, A. K., \& Kok, J. B. (2008). Numerical prediction of interaction between combustion, acoustics and vibration in gas turbines. Acoustics'08 (pp. 2749-2754). Paris: Proceedings of the Acoustics'08.

81. Pozarlik, A. K., Panara, D., Kok, J. B., \& van der Meer, T. H. (2008). Heat transfer in a recirculation zone at steady-state and oscillating conditions - the back facing step test case. 5th European Thermal-Sciences Conference, EUROTHERM2008. Eindhoven. 
82. Pozarlik, A., \& Kok, J. (2007). Numerical investigation of one- and two-way fluidstructure interaction in combustion systems. Proceedings of International Conference on Computational Methods for Coupled Problems in Science and Engineering, (pp. 610-613). Ibiza.

83. Putnam, A. (1971). Combustion driven oscillations in industry. New York: American Elsevier.

84. Rajaram, R., \& Lieuwen, T. (2002). Parametric Studies of Acoustic Radiation from Premixed Flames. AIAA, AIAA-2002-3864.

85. Rayleigh, J. (1878). The Explanation of Certain Acoustic Phenomena. Nature, 18 , 319-321.

86. Rhee, G., \& Sung, H. (2007). Enhancement of heat transfer in turbulent separated and reattaching flow by local forcing. Numerical Heat Transfer , 37, 733-753.

87. Rienstra, S. W., \& Hirschberg, A. (2001). An introduction to acoustics. Eindhoven: Technical Report, Technical University of Eindhoven.

88. Rijke, P. (1859). Notiz uber eine neue art, die luft in einer an beidenende offenen Rohre in schwingungen zu versetzen. Annalen der Physik, 107, 339-343.

89. Rotta, J. C. (1972). Turbulente Strömungen: eine Einführung in die Theorie und ihre Anwendung. Stuttgart: Teubner.

90. Russell, D. (2008). Retrieved from http://www.kettering.edu/ drussell/Demos/rad2/mdq.html

91. Sadeghi, M., \& Liu, F. (2005). Coupled Fluid-Structure Simulation for Turbomachinery Blade Rows. 43rd AIAA Aerospace Science Meeting and Exhibit,. Reno: Conference Proceedings.

92. Sengissen, A., Poinsot, T., Van Kampen, J., \& Kok, J. (2007). Response of a Swirled Non-premixed Burner to Fuel Flow Modulation. In Complex Effects in Large Eddy Simulations (Vol. 56, pp. 337-351). Berlin: Springer Berlin Heidelberg.

93. Steijger, O. M. (1998). Chemiluminescence and electrochemiluminescence : generation, optimization and application. Utrecht: $\mathrm{PhD}$ thesis, University of Utrecht.

94. Tam, C. K. (1995). Computational Aeroacoustics: Methods and Applications. AIAA , 33 (10), 1788-1796.

95. Thangam, S., \& Speziale, C. (1992). Turbulent flow past a backward facing step: a critical evaluation of two-equation models. AIAA, 30, 1314-1320.

96. The MathWorks, I. (2005). Matlab User Guide. The MathWorks, Inc. 
97. Tinga, T., Kampen, v. J., Jager, d. B., \& Kok, J. (2007). Gas Turbine Combustor Liner Life Assessment Using a Combined Fluid/Structural Approach. Journal of Engineering for Gas Turbines and Power, 129, 69-79.

98. Tsangalis, C., McLachlan, N., \& Trivailo, P. (2005). Fluid-structure interaction modelling of propellant combustion. ANZIAM J. , 47, 388-403.

99. Turns, S. R. (1996). An Introduction to combustion: concepts and applications. New York: McGraw-Hill.

100.UNFCCC. (1997). http://unfccc.int/kyoto_protocol/items/2830.php.

101.Valencia, A. (1997). Effect of pulsating inlet on the turbulent flow and heat transfer past a backward-facing step. Int. Comm. Heat Mass Transfer, 24, 1009-1018.

102. Van Kampen, J. (2006). Acoustic pressure oscillations induced by confined turbulent premixed natural gas flames. Enschede: $\mathrm{PhD}$ thesis, University of Twente.

103.Van Kampen, J., \& Kok, J. (2010). Characterisation of interaction between combustion dynamics and equivalence ratio oscillations in a pressurised combustor. International Journal of Spray and Combustion Dynamics , 2 (3), 219-252.

104.Versteeg, H. K., \& Malalasekera, W. (2007). An introduction to computational fluid dynamics : the finite volume method, 2nd edition. Harlow.

105.Vieser, W., Esch, T., \& Menter, F. (2002). Heat Transfer Predictions using Advanced Two-Equation Turbulence Models, CFX Validation Report. CFX-VAL 10/0602.

106.Vogel, J., \& Eaton, J. (1985). Combined Heat Transfer and Fluid Dynamics Measurements Downstream of a Backward-Facing Step. Journal of Heat Transfer , $107,922-929$.

107.Warnatz, J., Maas, U., \& Dibble, R. (2001). Combustion : physical and chemical fundamentals, modeling and simulation, experiments, pollutant formation. $3 r d$ edition. Berlin: Springer.

108. Wesseling, P. (2001). Principles of Computational Fluid Dynamics. Berlin: Springer.

109. Wilcox, D. C. (1986). Multiscale model for turbulent flows. AIAA .

110.Withag, J. A. (2007). Noise prediction for an oscillating lean premixed CH4/air flame. Enschede: MSc thesis, University of Twente.

111.Yoshikawa, H., Suga, T., \& Ota, T. (2005). Trubulent heat transfer around a downward-facing step - effects of step height. 6th World Conference on Experimental Heat Transfer, Fluid Mechanics and Thermodynamics. Miyagi, Japan. 
112.Ziada, S., \& Graf, H. (1998). Feedback control of combustion oscillations. Journal of Fluids and Structures, 12, 491-507.

113.Zienkiewicz, O. C., Taylor, R. L., \& Zhu, J. Z. (2005). The finite element method; its basis and fundamentals, 6th edition (Vol. 3). Oxford: Elsevier ButterworthHeinemann. 



\section{ACKNOWLEDGEMENTS}

Dear Reader, this is the last part of my $\mathrm{PhD}$ thesis. Here, I would like to show a gratitude to all people who have been involved in my life during these past few years. I realize that it is impossible to mention everybody, therefore thank all of you who have shared this time with me.

First of all, I would like to thank my supervisors: Prof. Theo van der Meer and Dr. Jim Kok. Both of them are excellent experts in the field but also very nice people. Jim was the person who gave me the opportunity to do my $\mathrm{PhD}$ in the EU project - Fluistcom. Jim, thank you for that but also for all the support you gave me during my study, for all interesting ideas which helped me in shaping this thesis; finally for the remarks and corrections that you made in my papers, presentations and of course this thesis. Thanks to you, the mystical but at the same time very interesting field of thermodynamics and gas turbines has been opened to me. I am very happy and proud that you were my supervisor and my mentor. Theo, there is one thing you should know... You are almost like father to all of us - ThW members. Thanks to you, working in this group is a real pleasure. I know that your doors are always open to me. Your opinion was always very important to me. I am grateful for all your remarks and corrections done to improve this thesis.

I am also very thankful to my project partners for all interesting and fruitful meetings which we had during my PhD research. My visits in Siemens, CERFACS, DLR, CIMNE, and QUB were very important experience for my study. Here, I would like to especially thank Postdoc/PhD's fellows from the project: Zinedine, Mauro, Daniele, Pavel and Jose. I am also very thankful to Jaap van Kampen and Rob Huls from Desire project for introducing me to the subject, discussions and good advice.

I would like to acknowledge all the ThW Group members but my special words of gratitude are addressed to: Prof. Gerrit Brem and Ir. Eddy Bramer (for familiarizing me with the biomass world), Dr. Genie Stoffels (for discussions about thermodynamics, sport, Dutch culture/grammar and geography, also for convincing me to run in the Batavierenrace - 
twice), Sally Kloost-Zimmerman van Woesik (for being such a nice and friendly person, and the best secretary ever), Henk-Jan Moed (for helping with the test rig and also for our morning meetings with cappuccino and talks about Dutch way of living, FC Twente, cars and Akita Inu). My Postdoc, PhD and MSc fellows (Uros, Timo, Marc, Anton V., Santhos, Nikola, Reddy, Lixian, Shanglong, Taha, Mehmet, Jerry, Miladin, Ashiq, Juan, Nutte, Ziad, Bram, Nijso, Marcel, Nick, Hans, Hubert, Bart, Arjen, Marten, Anton B., Desalegn, ...), I appreciate our discussions and jokes during coffee brakes, borrels and annual excursions. I have to mention however, some people particularly - my paranimfen: Jan - my roommate, excellent football player and man who take care of my cat (when I am abroad) and Maarten - musician and sportsmen who plays football, basketball and guitar (of course not at the same time). Both are great guys who tried to teach me Dutch; thank you for being such good friends. My roommates: Sjoerd, Ali and Anne; members of football teams Rotor and De Vlam (most of you I have already aforementioned, but have not mentioned yet - Rob and Denis, also here I would like to acknowledge those who played with us occasionally), basketball team, running and squash group - I have to say that I have spent great time with you at UT and after working hours. Additionally I would like to acknowledge: Bert Wolbert for his help with Siglab and LDV systems and Rudy Norder for having nice discussion about UT on late hours.

Zeljko, Karina, Izabela, Alex, Paweł, Agata, Łukasz, Marta, Michał, Emilka, Marcin, Mariusz and Ania, I am grateful for the time I have spent with you here and in Poland. Zeljko and Karina you have also special thanks for taking care of Kropeczka.

I would like to acknowledge my parents-in-law (Stanisław and Elżbieta), Paweł, grandmother Janina and whole family for their interest and support. I am grateful to my grandparents (Władysław and Sabina) for believing in me and encouraging me. Time which I have spent with you was wonderful. I am very sad that my grandfather Władysław passed away during my $\mathrm{PhD}$. I know that he will be always with me. I am thankful to my parents (Piotr and Rena). You are always in my life, supporting and helping me with important decisions. You have showed me how live and how to be a man. Thank you for everything you have done for me.

At the end I would like to thank my beloved wife, Magdalena. Madziu, you are always with me when I need you. Thank you for your support, good advice, encourage and your beautiful smile. You are my best friend and my love. I am happy with every day I spend with you. Thank you Słoneczko.

Bardzo dziękuje wszystkim, którzy byli ze mna podczas mojego doktoratu. Jestem wdzięczny za wsparcie $i$ zainteresowanie, ktore doświadczylem od moich teściów (Stanistawa $i$ Elżbiety), Pawła, babci Janiny i calej rodziny. Szczególne podziękowania chciałbym złożyć na ręce dziadka Władystawa i babci Sabiny, którzy zawsze wspierali i wierzyli we mnie. 
Dziękuje za wspaniały czas, który spędziłem z Wami. Bardzo żałuję, że mój dziadek Władysław nie doczekat tego momentu. Wiem jednak, że zawsze będzie ze mna. Jestem wdzięczny moim rodzicom (Piotrowi i Renie) za wsparcie, dodawanie otuchy i pomoc we wszystkich moich decyzjach życiowych. Jesteście zawsze w moim życiu. Pokazaliście mi jak zyć i jak być dobrym człowiekiem. Dziękuje za wszysto co robicie dla mnie.

Madziu, dziękuje Ci za to, że jesteś ze mna, że jesteś przy mnie kiedy Cię potrzebuję. Za Twoje wsparcie, dobre rady $i$ wspaniaty uśmiech. Jesteś moim przyjacielem $i$ moja miłościq. Jestem szczęśliwy z każdego dnia, który mogę spędzić z Tobq. Dziękuje Moje Stoneczko.

Artur, November $7^{\text {th }}, 2010$, Hengelo 